\title{
Expanding Reactivity in DNA-Encoded Library Synthesis via Reversible Binding of DNA to an Inert Quaternary Ammonium Support
}

Dillon T. Flood, ${ }^{1 \perp}$ Shota Asai, ${ }^{1 \perp}$ Xuejing Zhang,,${ }^{1,2}$ Jie Wang, ${ }^{1}$ Leonard Yoon, ${ }^{1}$ Zoë C. Adams, ${ }^{1}$ Blythe C. Dillingham, ${ }^{1}$ Brittany B. Sanchez,, Julien C. Vantourout, ${ }^{1}$ Mark E. Flanagan, ${ }^{3}$ David W. Piotrowski, ${ }^{3}$ Paul Richardson, ${ }^{4}$ Samantha A. Green, ${ }^{1}$ Ryan A. Shenvi, ${ }^{1}$ Jason S. Chen, ${ }^{\S}$ Phil S. Baran, ${ }^{1 *}$ Philip E. Dawson..$^{1 *}$

${ }^{1}$ Department of Chemistry, The Scripps Research Institute, 10550 North Torrey Pines Road, La Jolla, California 92037, United States

2The Institute of Drug Synthesis and Pharmaceutical Process, School of Pharmaceutical Sciences, Sun Yatsen University, Guangzhou 510006, China.

3Pfizer Medicinal Chemistry, Eastern Point Road, Groton, CT 06340, United States

4Pfizer Medicinal Chemistry, 10578 Science Center Drive, San Diego, CA 92121, United States

§Automated Synthesis Facility, Scripps Research, 10550 North Torrey Pines Road, La Jolla, CA 92037,

United States 


\section{Table of Contents}

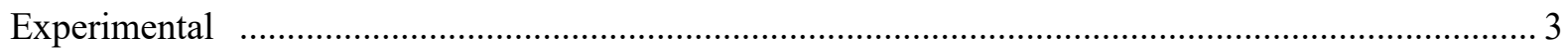

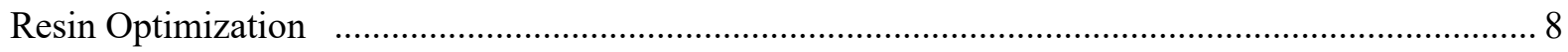

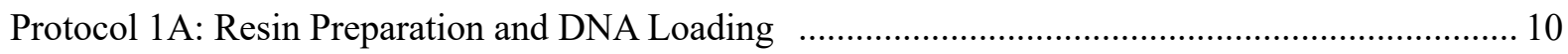

Graphical Supporting Information: Resin Preparation and DNA Loading (Protocol 1) ................... 11

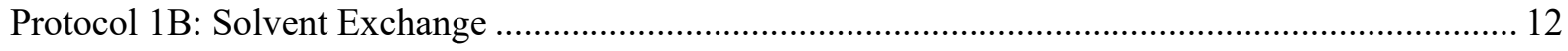

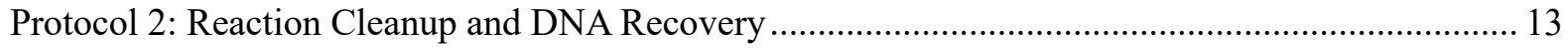

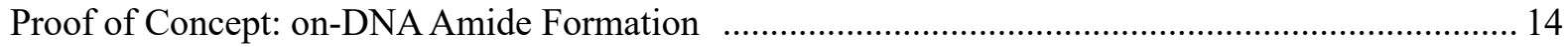

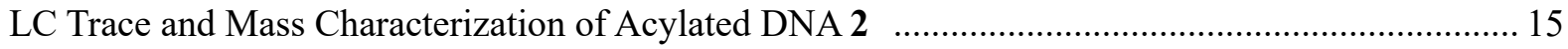

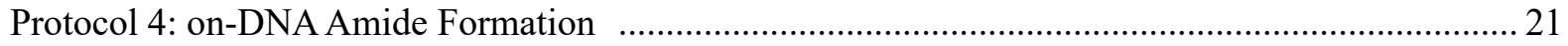

Troubleshooting: Frequently Asked Questions (Handling and Simple Reactions) ............................ 22

\section{DNA Decarboxylative sp2-sp3 Coupling}

Experimental Procedures and Product Characterization for DNA-bound (Het)Aryl Iodide, Aryl Bromide 23

Optimization of on-DNA Decarboxylative sp2-sp3 Coupling Reaction ............................................ 33

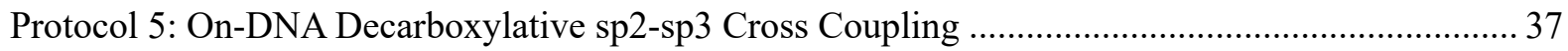

Graphical Supporting Information: Decarboxylative sp2-sp3 Coupling Reaction (Protocol 5) ......... 39

Troubleshooting: Frequently Asked Question (sp2-sp3 Coupling Reaction) .................................. 40

Characterization of on-DNA Decarboxylative sp2-sp3 Coupling Reaction Products 14-55 ............... 41

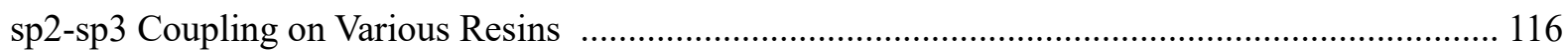

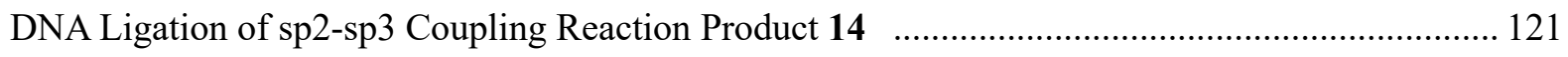

Failed Optimization of on-DNA Decarboxylative sp2-sp3 Coupling Reaction .............................. 123

\section{Electrochemical Amination}

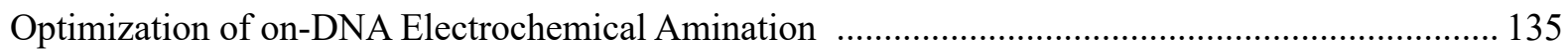

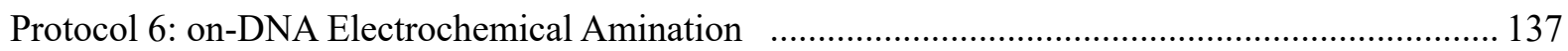

Graphical Supporting Information for on-DNA Electrochemical Amination (Protocol 6) ............. 139

Troubleshooting: Frequently Asked Questions (e-amination) ...................................................... 140

Characterization of on-DNA Electrochemical Amination Products 55-75 ................................... 141

\section{Reductive Amination}

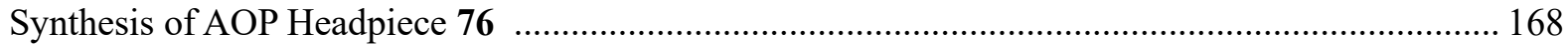

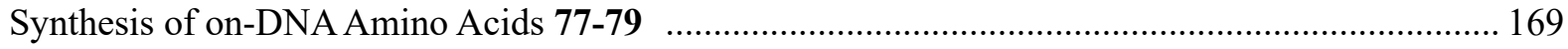


Experimental Procedures for on-DNA reductive Amination (General Procedure 7)........................ 173

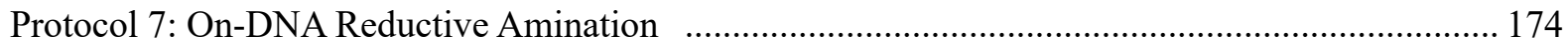

Graphical Supporting Information for on-DNA Reductive Amination (Protocol 7) ....................... 175

Troubleshooting: Frequently Asked Questions (on-DNA Reductive Amination) ............................ 176

Characterization of on-DNA Reductive Amination Products 80-100 ............................................. 177

\section{Towards Library Building}

DEL Rehearsal: Three Reaction Cycles Synthesis of 101 ............................................................. 219

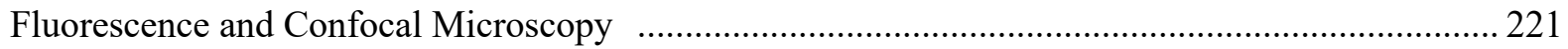

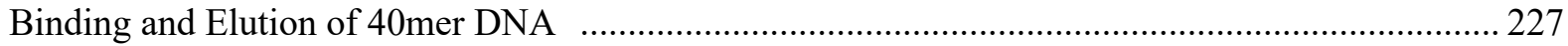

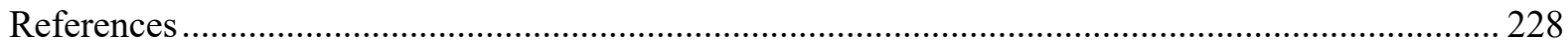




\section{General Experimental}

Tetrahydrofuran (THF), $\mathrm{N}, \mathrm{N}$-dimethylformamide (DMF), and dichloromethane $\left(\mathrm{CH}_{2} \mathrm{Cl}_{2}\right)$ and acetonitrile $\left(\mathrm{CH}_{3} \mathrm{CN}\right)$ were obtained by passing the previously degassed solvents through an activated alumina column. DMA was purchased from Sigma-Aldrich (lot \# SHBJ6878). DIC ( $N, N$ '-diisopropylcarbodiimide) was purchased from Oakwood. Deionized water was used in all the reactions, unless otherwise stated. $\mathrm{NiBr}_{2} \bullet$ diglyme was purchased from Sigma-Aldrich, Inc (lot \# 25024400). Zinc nanopowder was purchased from Strem Chemicals, Inc (lot \# A1804129). Pink catalyst was made by reported procedure. ${ }^{\mathrm{x}}$ All the other reagents were purchased at the highest commercial quality and used without further purification, unless otherwise stated.

DNA tags were obtained from IDT, Inc., Coralville, IA. T4 DNA Ligase was obtained from New England Biolabs, Ipswich, MA. The 10X DNA ligation buffer stock used in DNA ligation reactions was obtained from New England Biolabs, Ipswich, MA, and was composed as follows: $500 \mathrm{mM}$ Tris- $\mathrm{HCl} \mathrm{pH}$ 7.5, 100 $\mathrm{mM} \mathrm{MgCl}_{2}, 100$ mM DTT, 10 mM ATP. UltraPure ${ }^{\mathrm{TM}}$ Agarose was obtained from Invitrogen, Carlsbad, CA. 50X TAE Buffer (Tris-acetate-EDTA) was obtained from Thermo Fisher Scientific, Waltham, MA. SYBR $^{\mathrm{TM}}$ Safe DNA Gel Stain (10,000X) was obtained from Invitrogen, Carlsbad, CA. Gel Loading Dye, Purple (6X), no SDS was obtained from New England Biolabs, Ipswich, MA.

Yields under normal organic conditions refer to chromatographically and spectroscopically $\left({ }^{1} \mathrm{H}\right.$ NMR) homogeneous material, unless otherwise stated. TLC was performed using $0.25 \mathrm{~mm}$ E. Merck silica plates (60F-254), using short-wave UV light as the visualizing agent, and phosphomolybdic acid or $\mathrm{KMnO}_{4}$ and heat as developing agents. NMR spectra were recorded on Bruker DRX-600, DRX-500, and AMX-400 instruments and are calibrated using residual undeuterated solvent $\left(\mathrm{CHCl}_{3}\right.$ at $7.26 \mathrm{ppm}{ }^{1} \mathrm{H} \mathrm{NMR}, 77.16 \mathrm{ppm}$

${ }^{13} \mathrm{C}$ NMR). The following abbreviations were used to explain multiplicities: $\mathrm{s}=$ singlet, $\mathrm{d}=$ doublet, $\mathrm{t}=$ triplet, $\mathrm{q}=$ quartet, $\mathrm{m}=$ multiplet, $\mathrm{br}=$ broad. Column chromatography was performed using E. Merck silica gel (60, particle size $0.043-0.063 \mathrm{~mm}$ ), and preparative TLC was performed on Merck silica plates (60F254). High-resolution mass spectra (HRMS) were recorded on Agilent LC/MSD TOF mass spectrometer by electrospray ionization time of flight reflectron experiments. Melting points were recorded on a FisherJohns 12-144 melting point apparatus and are uncorrected. 
DNA headpiece materials. Three types of DNA headpieces were used in this paper and their structures are shown below. DNA headpiece (S1) (hairpin, Figure 1) was purchased from IDT. DNA headpiece (5'/5Phos/GAGTCA/iSp9/iUniAmM/iSp9/TGACTCCC-3', Figure 2) and DNA headpiece with chemical spacer (will call AOP-headpiece in this supporting information, Figure 3) were received from Pfizer. Both headpieces are received as aqueous solution $(20 \mathrm{mM})$.

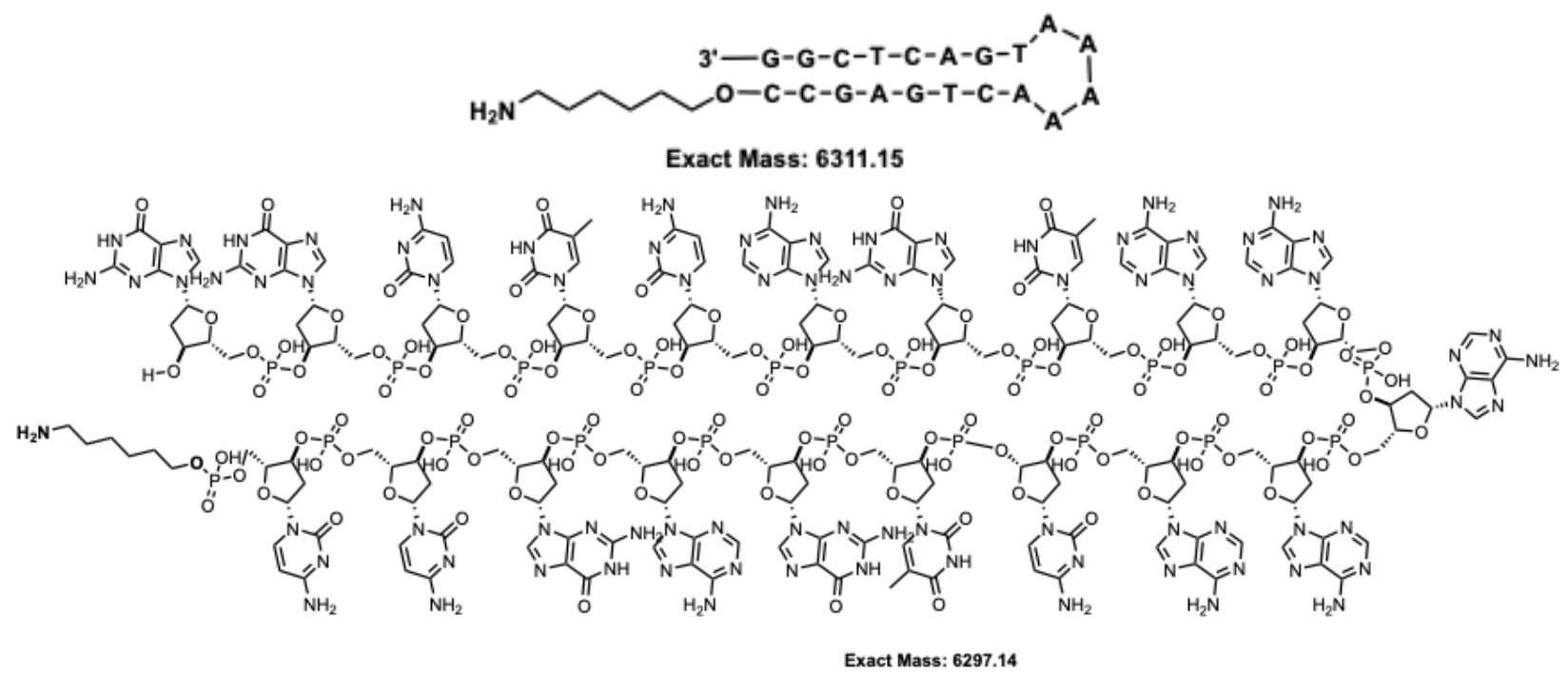

Figure 1. (SI-1) DNA Hairpin (Mock Headpiece): 5'-/5AmMC6/ CCGAGT CAA AAA TGA CTC GG-3' 


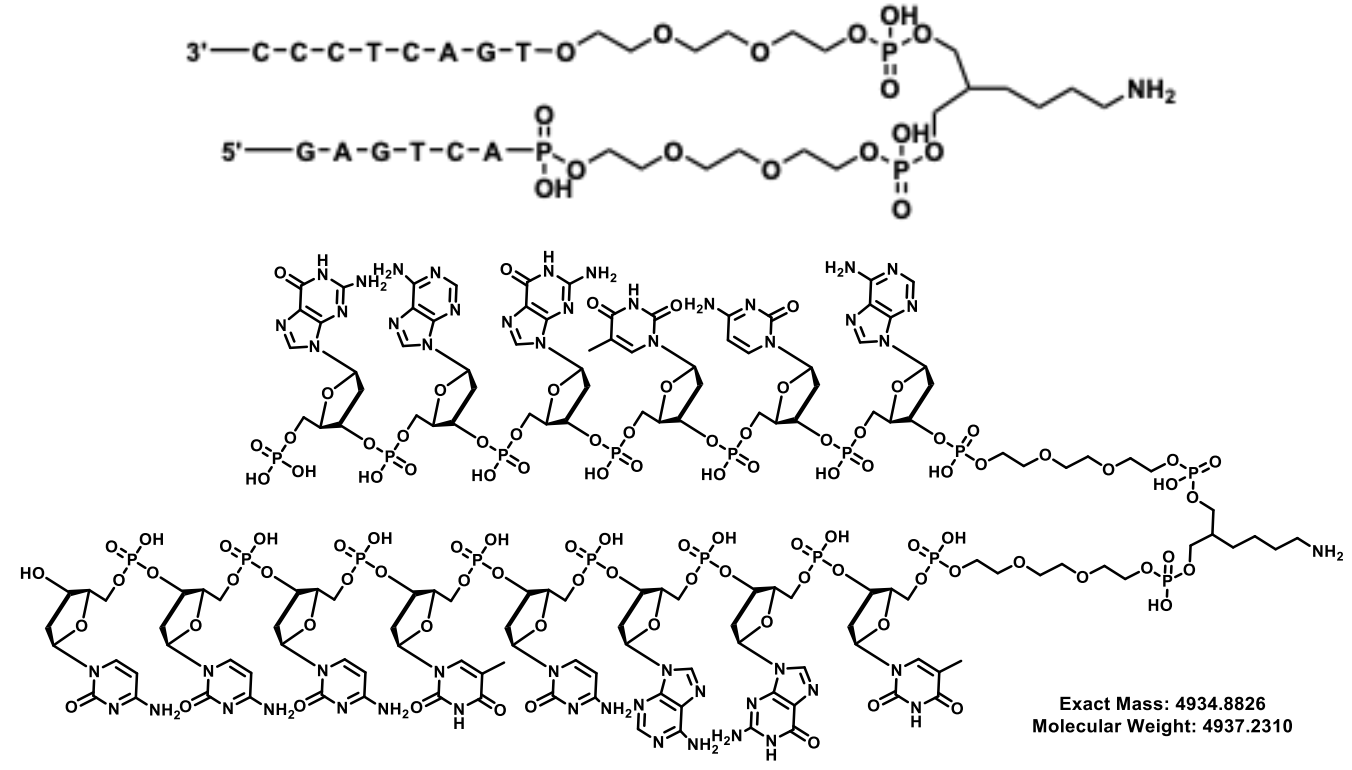

Figure 2. (SI-3) Headpiece: 5'-/5Phos/GAGTCA/iSp9/iUniAmM/iSp9/TGACTCCC-3'.
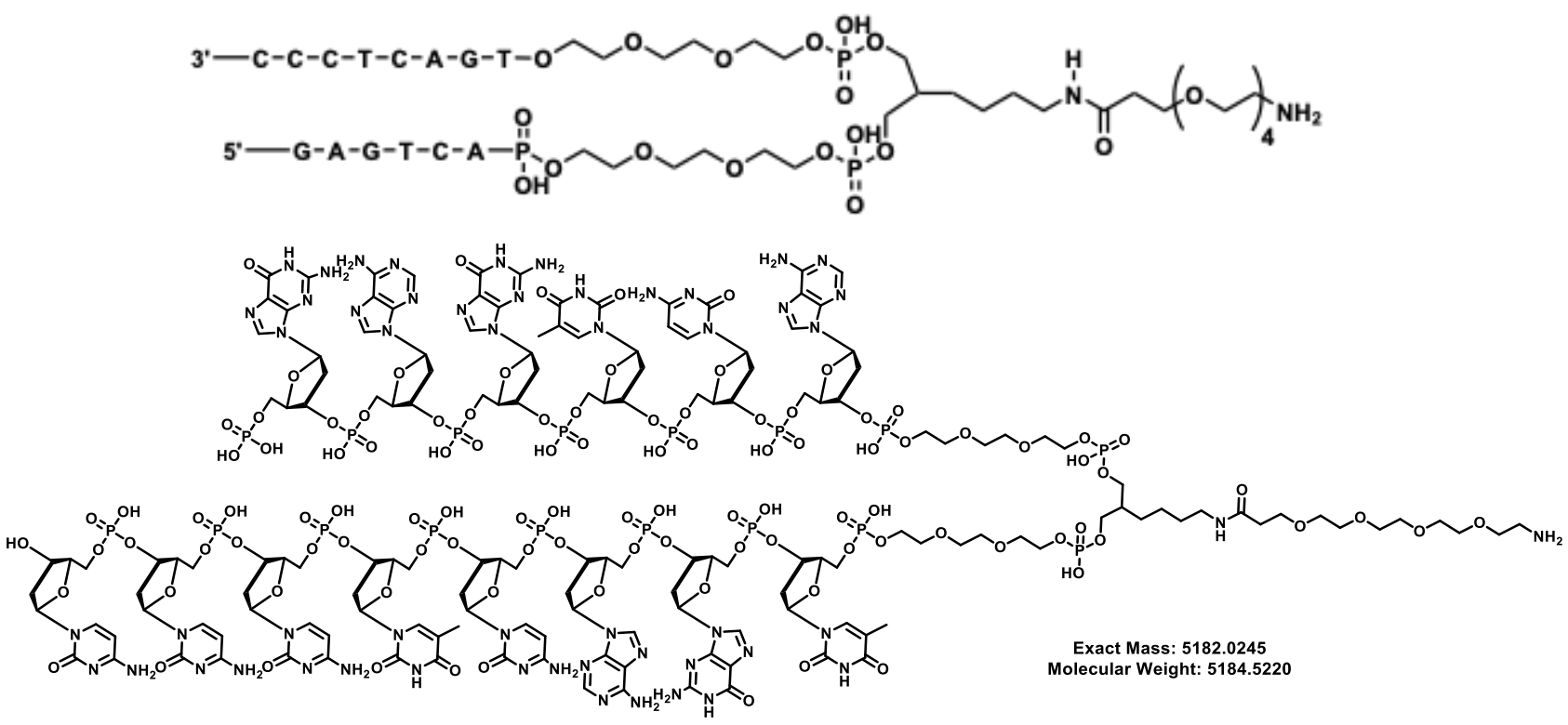

Figure 3.(66) AOP-headpiece with chemical spacer. 


\section{Synthesis of Redox-active Esters}

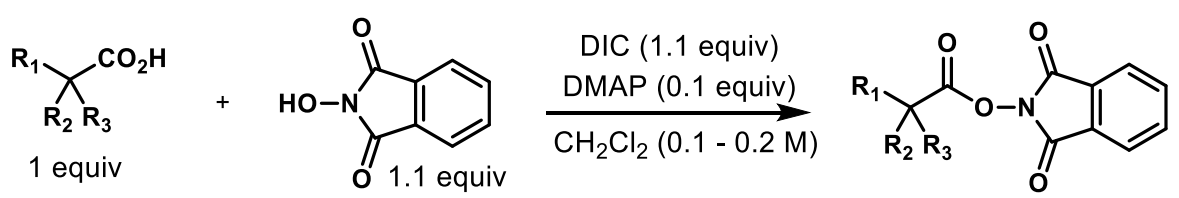

Redox-active esters were prepared according to the previously reported procedure. In short, a round-bottom flask or culture tube was charged with carboxylic acid (1.0 equiv), $N$-hydroxy-phthalimide (1.1 equiv) and $\operatorname{DMAP}(0.1$ equiv). Dichloromethane was added $(0.1-0.2 \mathrm{M})$ followed by DIC (1.1 equiv), and the mixture was allowed to stir vigorously until the acid was consumed (determined by TLC). Typical reaction times were between $0.5 \mathrm{~h}$ and $12 \mathrm{~h}$. The mixture was filtered (over Celite, $\mathrm{SiO}_{2}$, or through a fritted funnel) and rinsed with additional $\mathrm{CH}_{2} \mathrm{Cl}_{2} / \mathrm{Et}_{2} \mathrm{O}$. The solvent was removed under reduced pressure, and purification by column chromatography (and recrystallization, if necessary) afforded the corresponding redox-active ester.

We have previously reported the synthesis of redox-active esters shown below. Please see these references for graphical supporting information on the synthesis of redox-active esters.<smiles>O=C(Nc1ccccc1)C1CCN(C(=O)c2ccccc2)CC1</smiles><smiles>CCNC(=O)C1CCN([As])CC1</smiles><smiles>CCCCNC(=O)C1CCN(C(=O)c2ccccc2)CC1</smiles><smiles>CCCNC(=O)C1CCC(F)CC1</smiles><smiles>O=C(Nc1ccccc1)C1CCOCC1</smiles><smiles>O=C(Nc1ccccc1)C1CCCCC1</smiles><smiles>CCPNC(=O)C1C(C)(C)C1(C)C</smiles><smiles>CCCCCNC(=O)C1CC2C=CC1C2</smiles><smiles>CCCNC(=O)CC1CCCC1</smiles><smiles>CCNC(=O)CCc1ccc(F)cc1</smiles><smiles>O=C(ON[In])C1Cc2ccccc2C1</smiles><smiles>CCCNOC(=O)Cc1ccccc1Nc1c(Cl)cccc1Cl</smiles><smiles>COC(=O)CCCCC(=O)ONP</smiles><smiles>O=C(CCCc1ccccc1)ON=P</smiles> 


\section{Analysis of DNA Headpieces (Method LCMS-II)}

DNA concentration: DNA samples subjected to HPLC-MS analysis were prepared as $0.1 \mathrm{mM}$ in $\mathrm{H}_{2} \mathrm{O}$, assuming $100 \%$ of DNA total recovery after reaction.

Analysis: Analysis: One microliter of the DNA solution was analyzed on a Waters I-Class LC with a Waters BEH C18 column $(2.1 \times 55 \mathrm{~mm}, 1.7 \mu \mathrm{m}, 130 \AA)$ using a gradient of $114 \mathrm{mM}$ HFIP and $14 \mathrm{mM} \mathrm{Et}_{3} \mathrm{~N}$ in water (A) and methanol (B) $(0.3 \mathrm{~mL} / \mathrm{min}, 10-26 \% \mathrm{~B}$ over 10 minutes $)$ at $60^{\circ} \mathrm{C}$. The yield was determined by calculating the percentage of UV absorbance at $260 \mathrm{~nm}$ corresponding to the product peak, ignoring potential UV absorption coefficient differences between DNA products and assuming $100 \%$ mass recovery. Peak identities were determined by ESI- using the 3- ion.

Deconvolution: data visualization and integration was performed with Mass Lynx V4.1 software.

Yield determination: the yields of on-DNA products were determined from UV absorbance trace (260 nm) peak area using the equation: Yeild $\%=\mathrm{UV}($ prod $) / \mathrm{UV}$ (total DNA recovered), while ignoring UV coefficient difference for all DNA products and assuming $100 \%$ of DNA total recovery. While determining yields, any non-oligo material (as determined by close examination of mass spectra) that absorbed UV $260 \mathrm{~nm}$ was subtracted out of the final yield calculation. These peaks were determined to not contain oligo material when a DNA indicative mass spectra was not observed, and are usually attributed to small molecules not removed during the ethanol precipitation. These peaks were generally found at or before 3 minutes retention time during analysis.

MS deconvolution: while multi-charged (negative) mass was observed, triply charged mass was determined to be base peak in all cases. Observed $\mathrm{m} / \mathrm{z}$ could be calculated as $\mathrm{m} / \mathrm{z}=[\mathrm{M}-\mathrm{z}] / \mathrm{z}$. 


\section{Resin Optimization}

Optimal resin was discovered by screening multiple reversed phase (Strata-X, SDBL, and $\mathrm{C}_{18}$ Silica) and anion exchange (Strata-XA) resins from Phenomenex. Reversed phase resins were swelled in 1:1 DNA Buffer A (water containing $120 \mathrm{mM}$ HFIP and $20 \mathrm{mM}$ TEA):MeOH and anion exchange resins were swelled in 1:1 PBS:MeCN. Aliquots $(100 \mu \mathrm{L})$ of swollen resin were taken via micropipette and transferred into a 2 $\mathrm{mL}$ Eppendorf tube. DNA Buffer A or PBS were added $(1 \mathrm{~mL})$ to reversed phase or anion exchange resins, respectivly top wash away organic solvents. The resin slurries were spun down in a benchtop centrifuge and the supernatant was removed by glass pipette and discarded.

Mock DNA head piece ( $25 \mathrm{nmol}$ ) S1 was dissolved to $0.1 \mathrm{mM}$ in water containing $120 \mathrm{mM}$ HFIP and 20 $\mathrm{mM}$ triethylamine or $1 \mathrm{x}$ PBS (commercial) and added to reversed phase resins or anion exchange resins respectively. The resins slurries were vortexed (15 seconds) and allowed to shake on an orbital shaker for 15 minutes.

After 15 minutes the resin slurries were spun down, and the supernatant was carefully removed and by micropipette and saved. These supernatant samples were added to an HPLC vial and sent for analysis. The resin beds were then washed (solution added, vial vortexed, vial spun down, and supernatant removed) with $1 \mathrm{~mL}$ of either DNA Buffer A (reversed phase resins) or PBS (anion exchange resin).

The DNA was then eluted from the resin beds by the addition of $150 \mu$ L HPLC Buffer B (1:1 DNA Buffer $\mathrm{A}: \mathrm{MeOH})$ to the revered phase resins and percholrate buffer $\left(1 \mathrm{M} \mathrm{NaClO}_{4}, 20 \% \mathrm{MeOH}, 40 \mathrm{mM}\right.$ Tris $\mathrm{pH}$ 8.5 , in water) to the anion exchange resins. The resin slurries were vortexed and placed on a shaker for 10 minutes. After ten minutes the slurries were spun down, the supernatant carefully removed by micropipette and collected in $1.5 \mathrm{~mL}$ Eppendorf tubes. The above process was repeated 2 more times (total of 3 washes) and the collected elution solutions were again spun down and transferred to $2 \mathrm{~mL}$ Eppendorf tubes to which $10 \% \mathrm{v} / \mathrm{v} 5 \mathrm{M} \mathrm{NaCl}$ and 3 volumes of cold EtOH were added sequentially. The samples were vortexed and allowed to sit at $-20^{\circ} \mathrm{C}$ for 30 minutes. The samples were then spun down (10,000 RPM) for 10 minutes, the EtOH was discarded and the DNA pellet was dried by vacuum. The DNA was redissolved with the addition of $0.25 \mathrm{~mL}$ water and sent for analysis. 


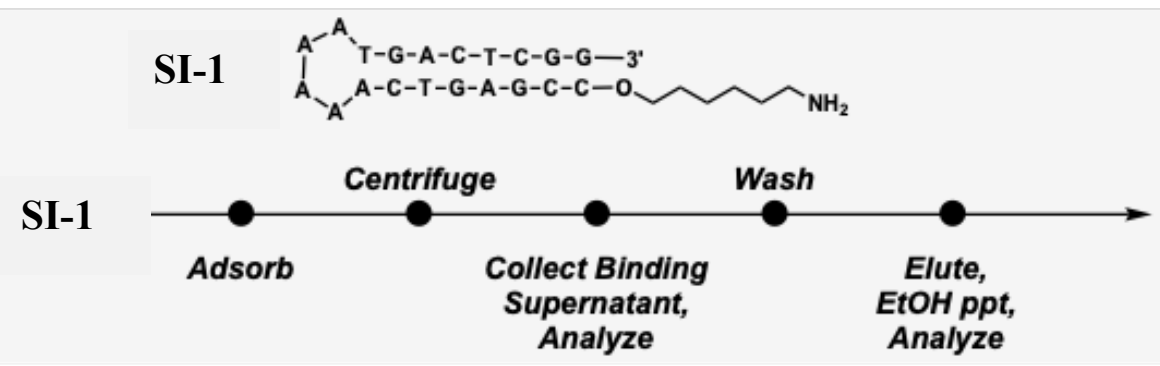

\section{Anion Exchange Resin}
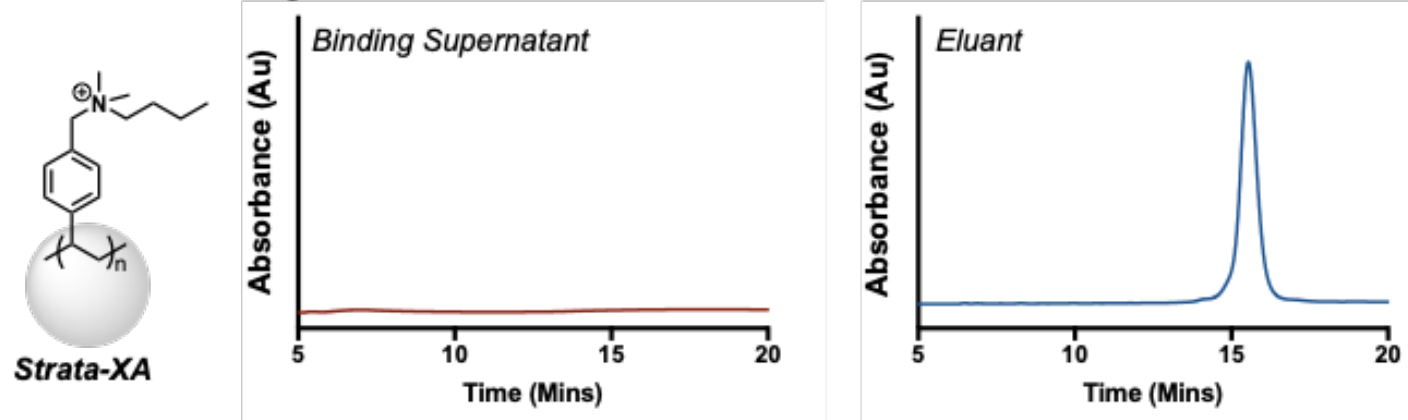

\section{Reversed Phase Resin}
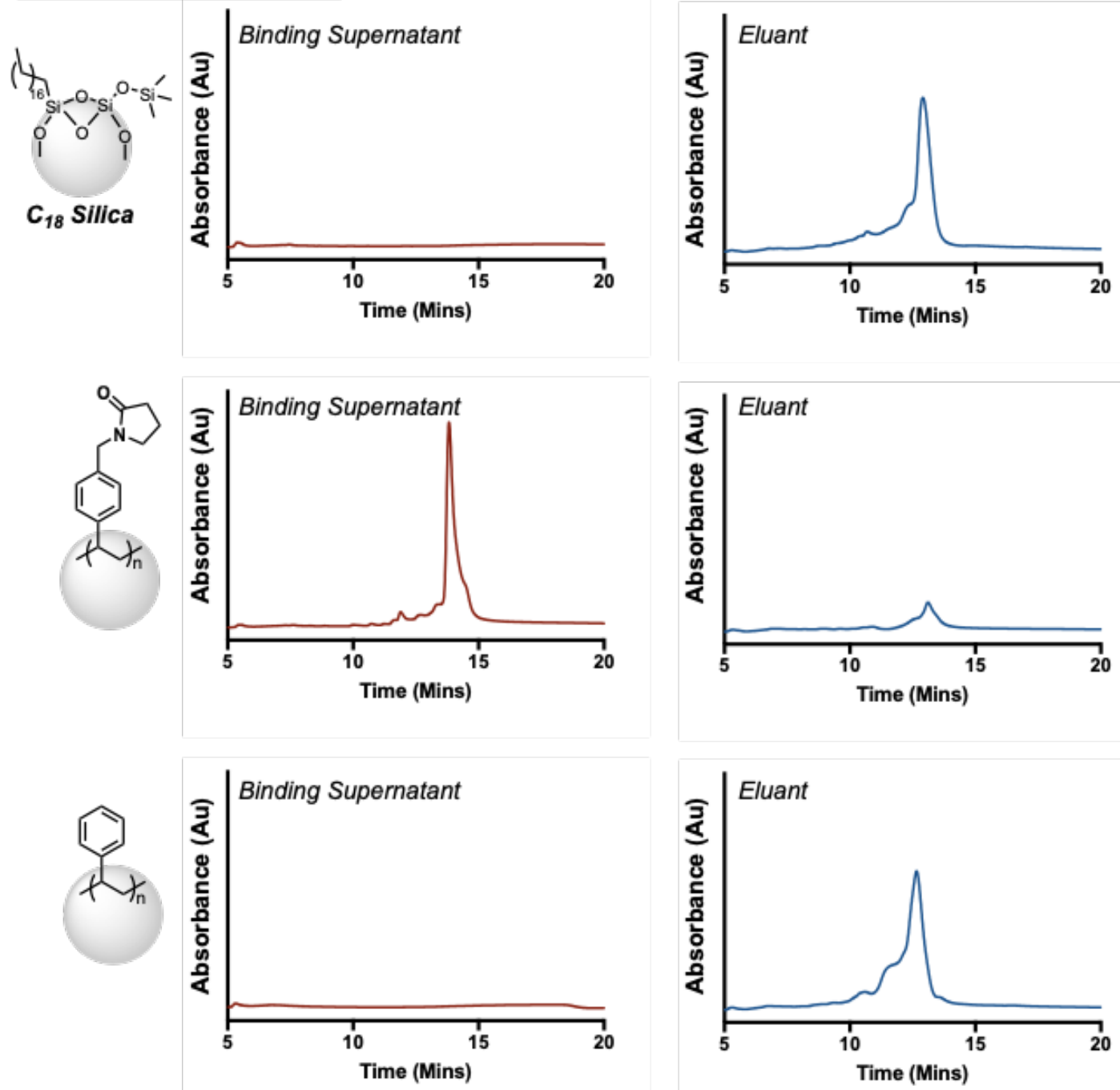


\section{Protocol 1A: Resin Preparation and DNA Loading}

\section{Resin Preparation and DNA Loading}

1. Cut the tip off of a $1 \mathrm{~mL}$ pipette tip and transfer $100 \mu \mathrm{L}$ swelled resin with an adjustable volume pipette-Phenomenex Strata-XA-(in 1:1 phosphate buffered saline (PBS):ACN) to a $2 \mathrm{~mL}$ (cylindrical) microcentrifuge tube.

2. Alternatively weigh $15-25 \mathrm{mg}$ of dry resin into a $2 \mathrm{~mL}$ microcentrifuge tube and add $500 \mu \mathrm{L} 1: 1$ PBS:ACN for 30 minutes to swell the resin.

3. Add $1000 \mu \mathrm{L}$ PBS to the resin and vortex.

4. Spin down the resin slurry and aspirate the resin bed (by sucking off the supernatant with a glass pipet).

5. Add $100 \mu \mathrm{L}$ of PBS to the resin bed, and then add $10 \mathrm{nmol}$ DNA from a stock solution in water.

6. Vortex the resin bed and allow to sit for 5-15 $\mathrm{min}$.

7. Spin down the resin bed and aspirate the supernatant with a pipette.

8. Add $500 \mu \mathrm{L}$ DMA (or other water miscible organic solvent which is suitable for the subsequent reaction) to the resin bed and vortex. Spin down the slurry and remove excess solvent.

Note: Strata-XA $33 \mu \mathrm{M}$ resin is only available in prefilled SPE tubes which can be broken apart. Bulk resin is available under the label Sepra ZT-SAX $30 \mu \mathrm{m}$.

Note: Phenomonex also produces a larger particle sized resin for use with larger analytes. For large oligos (>100 bp) Strata-XAL $100 \mu \mathrm{m}$ is the preferred support.

Note: "Washing the resin" refers to adding 500 to $1000 \mu \mathrm{L}$ of solvent to the resin, vortexing (30 sec), spinning the resin down, and removing the supernatant with a pipette. 


\section{Graphical Supporting Information Resin Preparation and DNA Loading (Protocol 1)}

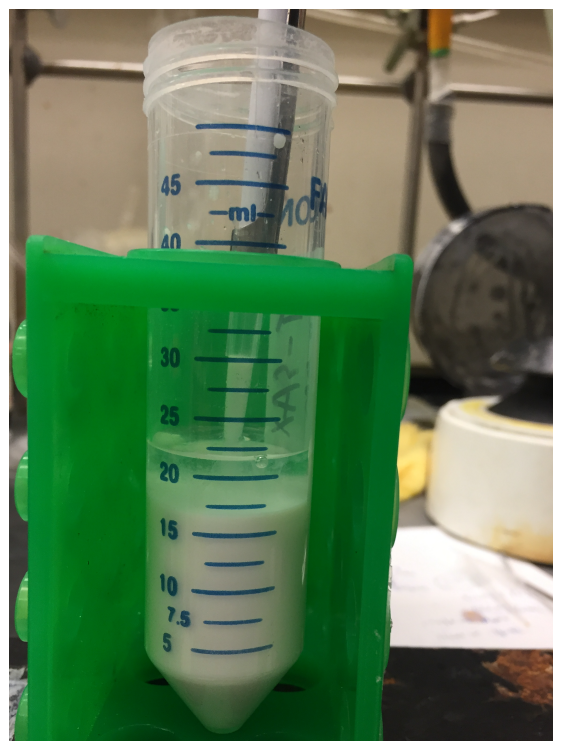

Taking $100 \mu \mathrm{L}$ aliquot from tube with swollen and settled resin in 1:1 PBS:MeCN

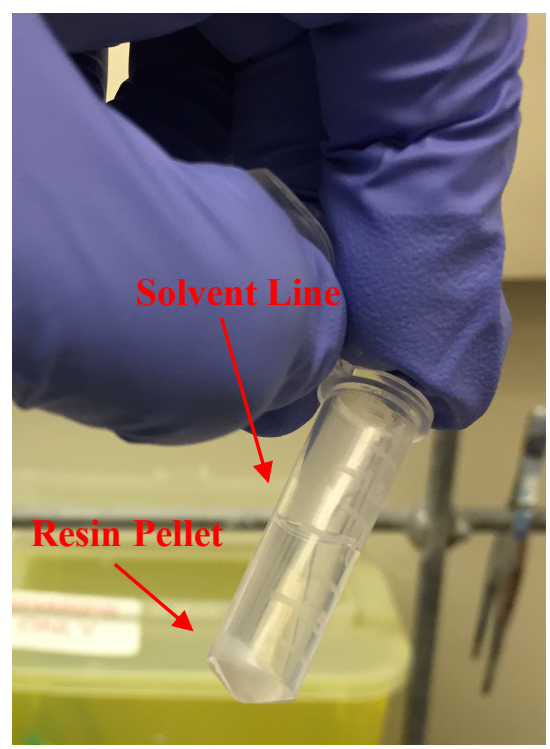

Resin pellet after centrifugation.

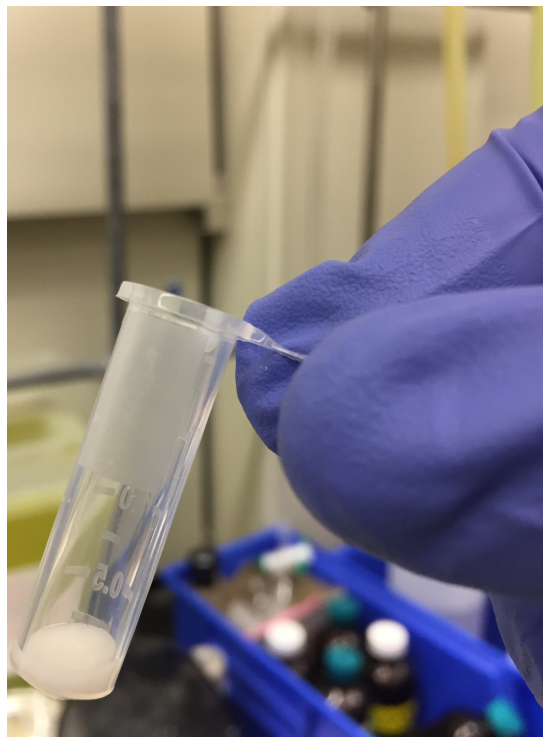

Taking $100 \mu \mathrm{L}$ aliquot of swollen resin.

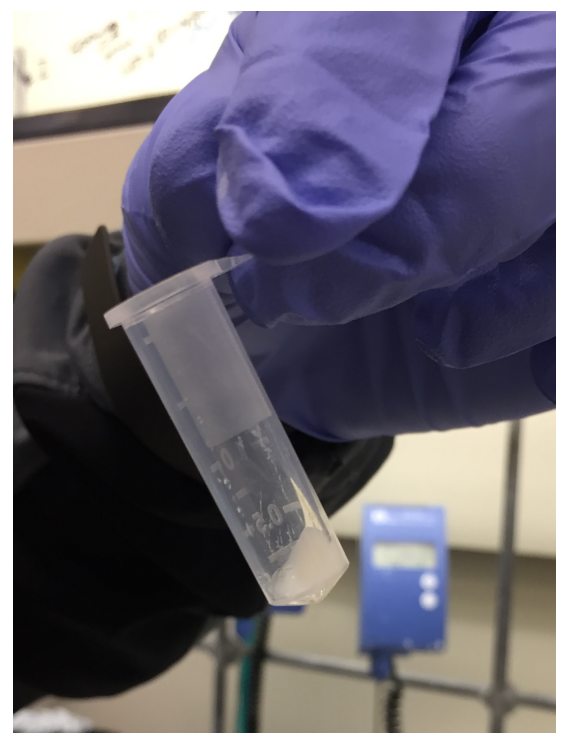

Resin pellet after removing PBS supernatant by glass pipette.

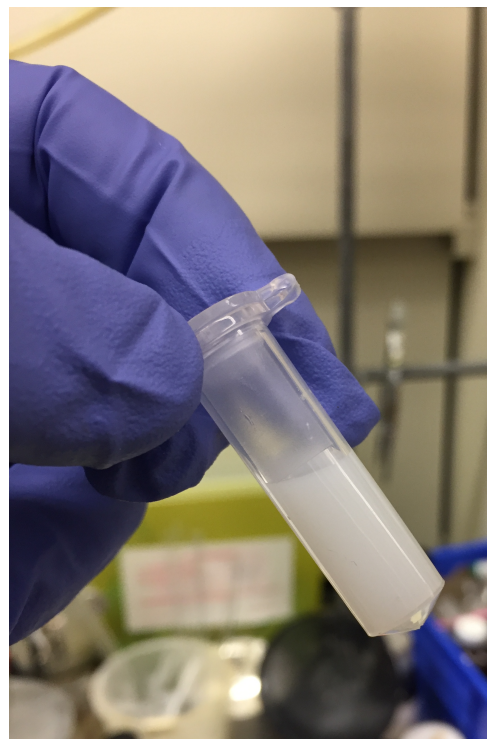

Resin slurry after addition of 1 $\mathrm{mL}$ of PBS and vortexing.

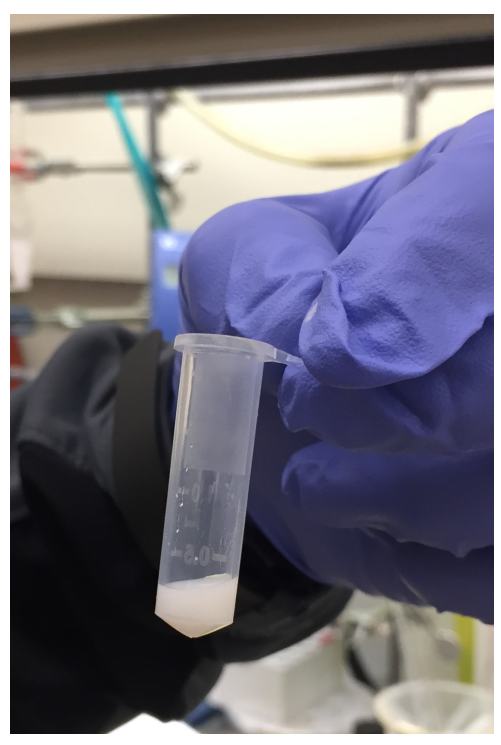

Resin slurry after addition of 100 $\mu \mathrm{L}$ PBS and 10 nmol DNA. 


\section{Protocol 1B: Solvent Exchange}

Solvent Exchange Protocol

1. When transferring resin into a water-immiscible organic solvent:

2. Wash resin with a water miscible organic solvent (DMA, ACN, and THF are all acceptable).

3. Add $1 \mathrm{~mL}$ solvent to resin bed containing residual aqueous solution.

4. Vortex for 30 seconds.

5. Spin down the resin and discard supernatant.

6. This process may be repeated depending on the tolerable levels of residual water in the reaction mixture.

7. Wash the resin with water immiscible organic solvent (toluene and DCM work well with our resin while $100 \%$ DCE is incompatible and causes resin collapse) which will be the same as the reaction to be performed.

8. Add $1 \mathrm{~mL}$ solvent.

9. Vortex for 30 seconds.

10. Spin down the resin and discard supernatant. 


\section{Protocol 2: Reaction Cleanup and DNA Recovery}

Reaction Clean up and DNA recovery

1. Add $1000 \mu \mathrm{L}$ of DMA (or other water miscible solvent-THF, DMA or ACN) to reaction mixture, remove stir bar, vortex, spin down, and remove supernatant.

a. Optional: Wash resin bed with $500 \mu \mathrm{L}$ DMA, THF or ACN:

2. Add solvent, vortex, spin down, and aspirate (you may want to repeat this step if performing sequential reactions).

3. Wash resin bed with $1000 \mu \mathrm{L}$ PBS: vortex, spin down, and aspirate.

4. Add $150 \mu \mathrm{L}$ Elution buffer to the resin vortex and agitate for 10 minutes.

5. Elution buffer $=1 \mathrm{M} \mathrm{NaClO}_{4}, 40 \mathrm{mM}$ Tris $\mathrm{pH} 8.5,20 \% \mathrm{MeOH}$.

6. Spin down mixture and collect $100 \mu \mathrm{L}$ eluent in separate microcentrifuge tube.

7. Then add $100 \mu \mathrm{L}$ elution buffer to the resin bed, then vortex and agitate for 10 minutes.

8. Spin down mixture and collect $100 \mu \mathrm{L}$ elution buffer in separate microcentrifuge tube.

9. Repeat step 6 and 7, but collect $150 \mu \mathrm{L}$ of elution buffer after the third elute volume. (3 elution volumes total, $350 \mu \mathrm{L}$ ).

10. Spin down the collected elute buffer to rid the solution of solid particulates and resin. Transfer the solution to a $2 \mathrm{~mL}$ microcentrifuge tube.

11. Add $5 \mathrm{M} \mathrm{NaCl}(10 \% \mathrm{vol} / \mathrm{vol})$, and fill tube to the $2 \mathrm{~mL}$ mark with cold $\mathrm{EtOH}$.

12. Place in freezer for $>20$ mins. And centrifuge for $>20$ mins.

13. Redissolve in water and analyze by HPLC-MS. 


\section{Proof of Concept: Amide Formation (Protocol 3)}

Proof of concept was demonstrated by performing an amide reaction on resin bound DNA in differing organic solvents. DNA SI-1 adsorbed to resin $(100 \mu \mathrm{L})$, by procedure outlined above, and washed with 1 $\mathrm{mL}$ of various organic solvents. A coupling solution of DIC (100 mM), NHS (100 mM), and bromophenylacetic acid $(100 \mathrm{mM})$ were prepared in various solvents. To the washed resin beds, a small stirbar, $250 \mathrm{uL}$ of coupling solution, and TEA $(6 \mu \mathrm{L}, \sim 150 \mathrm{mM})$ were added. The reaction tubes were allowed to stir for 2 hours at which point the stir bar was removed, the tube spun down and the reaction mixture supernatant discarded. Each reaction mixture was washed with organic solvent (1 mL), and PBS (1mL). The resin was then eluted, and the elution mixture was EtOH ppt. The resulting samples were dissolved to $0.1 \mathrm{mM}$ and analyzed by HPLC-MS.

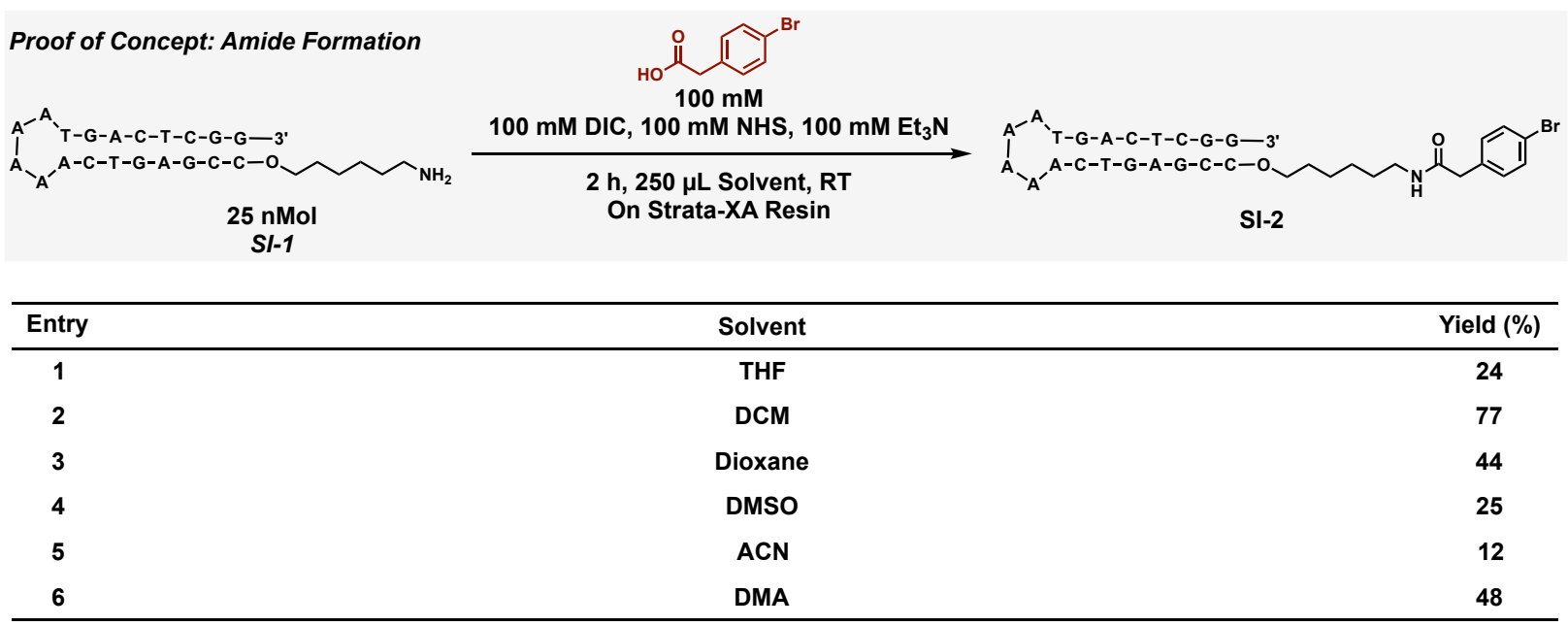




\section{HPLC Trace and Mass Characterization for Acylated DNA SI-2}

Entry 1: THF

\section{LC Trace and Mass of SI-2}

Following General Procedure 3 with SI-1

Yield: $24 \%$

Exact mass: 6507.1

Triply charged mass [M-3]/3, calculated: 2168.03; observed 2168.38
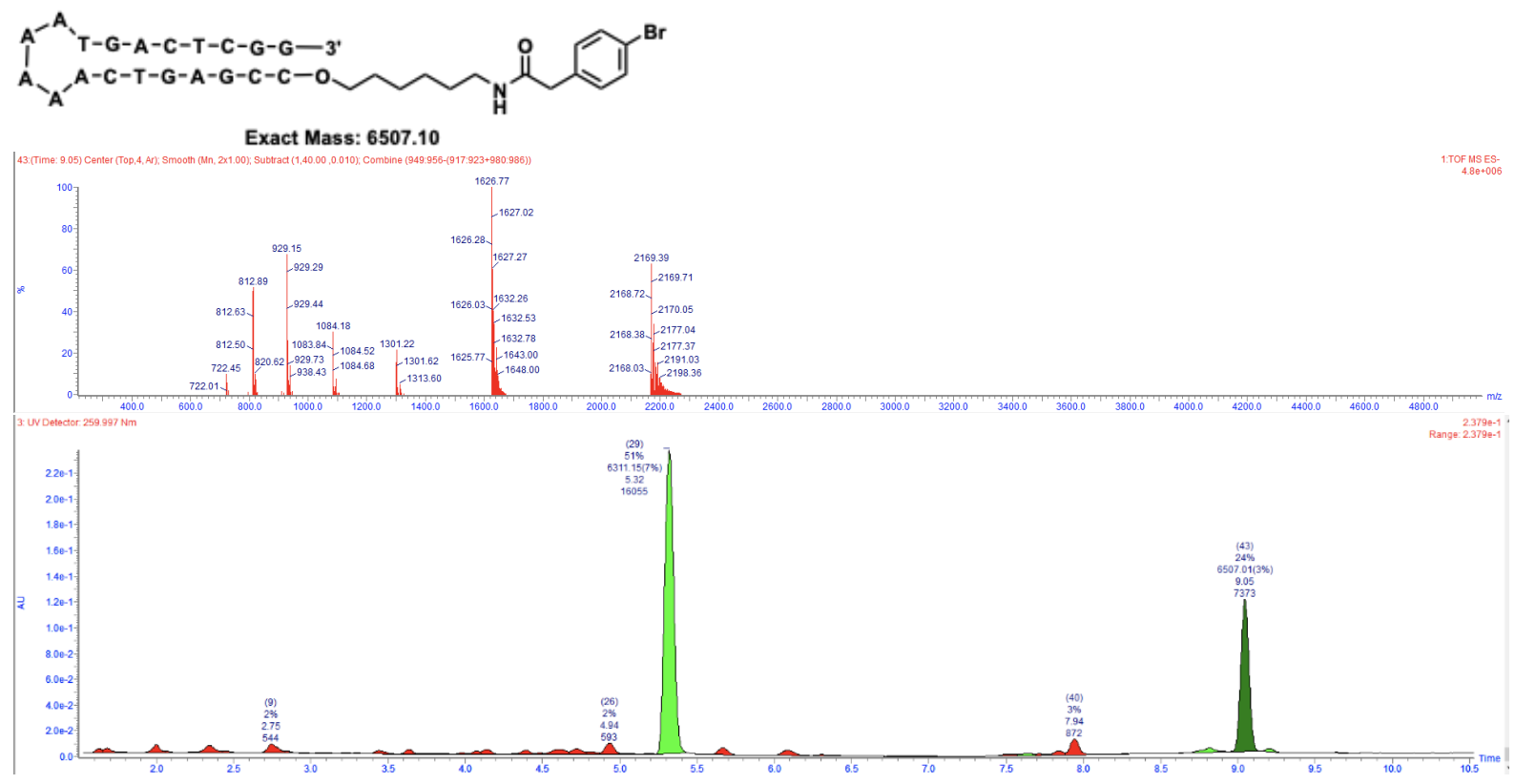


\section{Entry 2: DCM}

\section{LC Trace and Mass of SI-2}

\section{Following General Procedure 3 with SI-1}

Yield: $77 \%$

Exact mass: 6507.1

Triply charged mass [M-3]/3, calculated: 2168.03; observed 2168.38
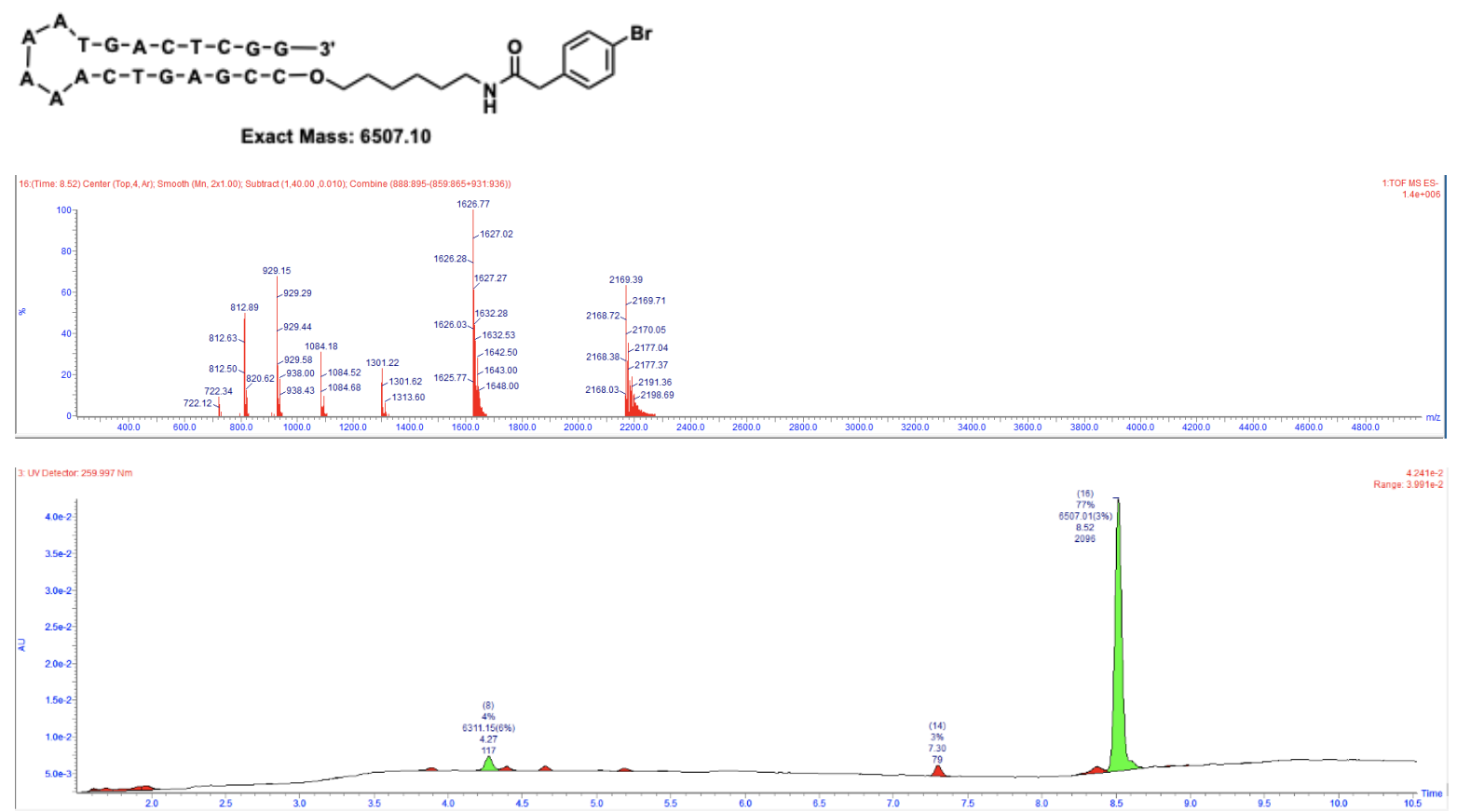
Entry 3: 1,4-Dioxane

\section{LC Trace and Mass of SI-2}

Following General Procedure 3 with SI-1

Yield: $44 \%$

Exact mass: 6507.1

Triply charged mass [M-3]/3, calculated: 2168.03; observed 2168.37
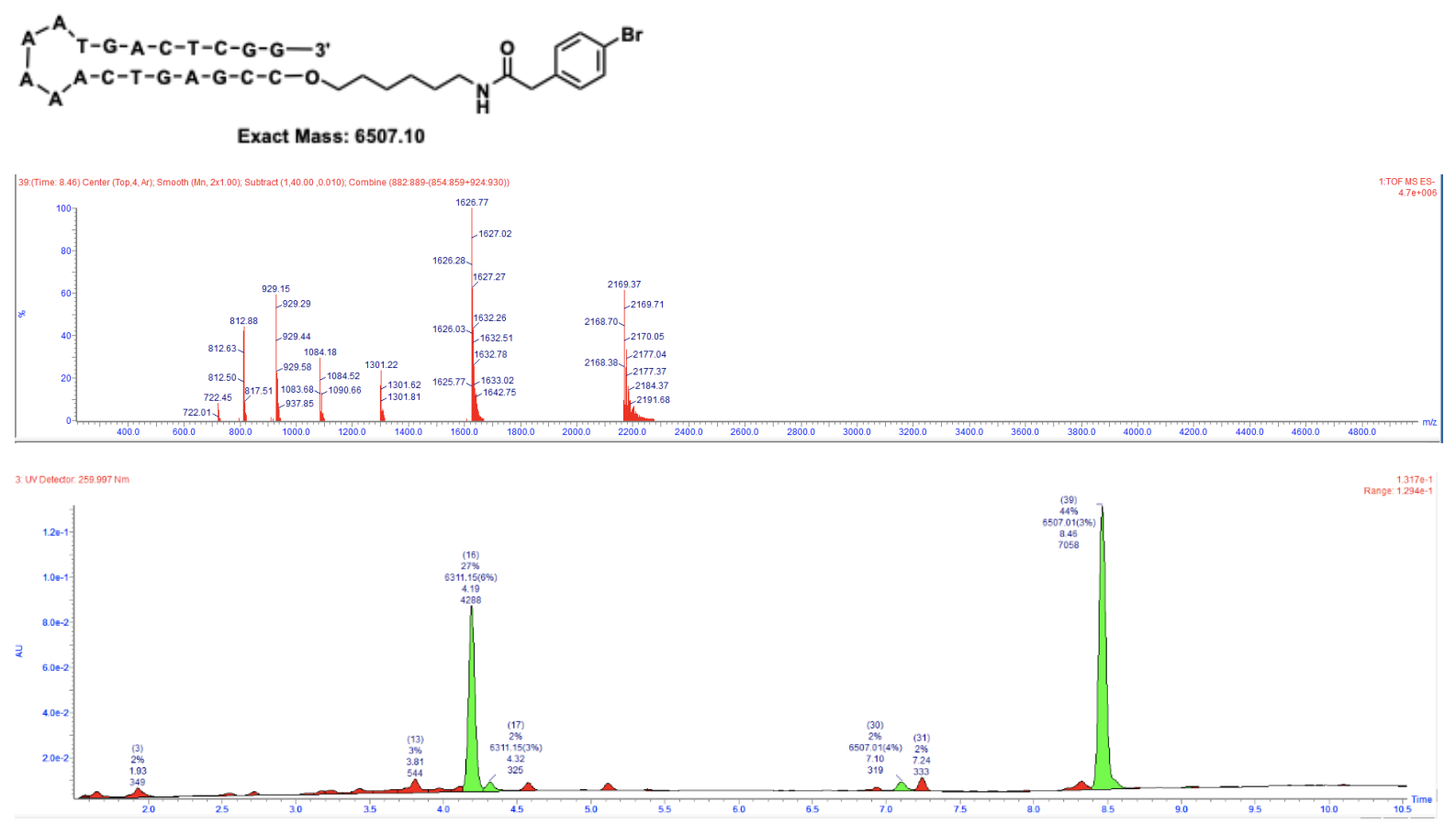
Entry 4: DMSO

\section{LC Trace and Mass of SI-2}

Following General Procedure 3 with SI-1

Yield: $25 \%$

Exact mass: 6507.1

Triply charged mass [M-3]/3, calculated: 2168.03; observed 2168.39
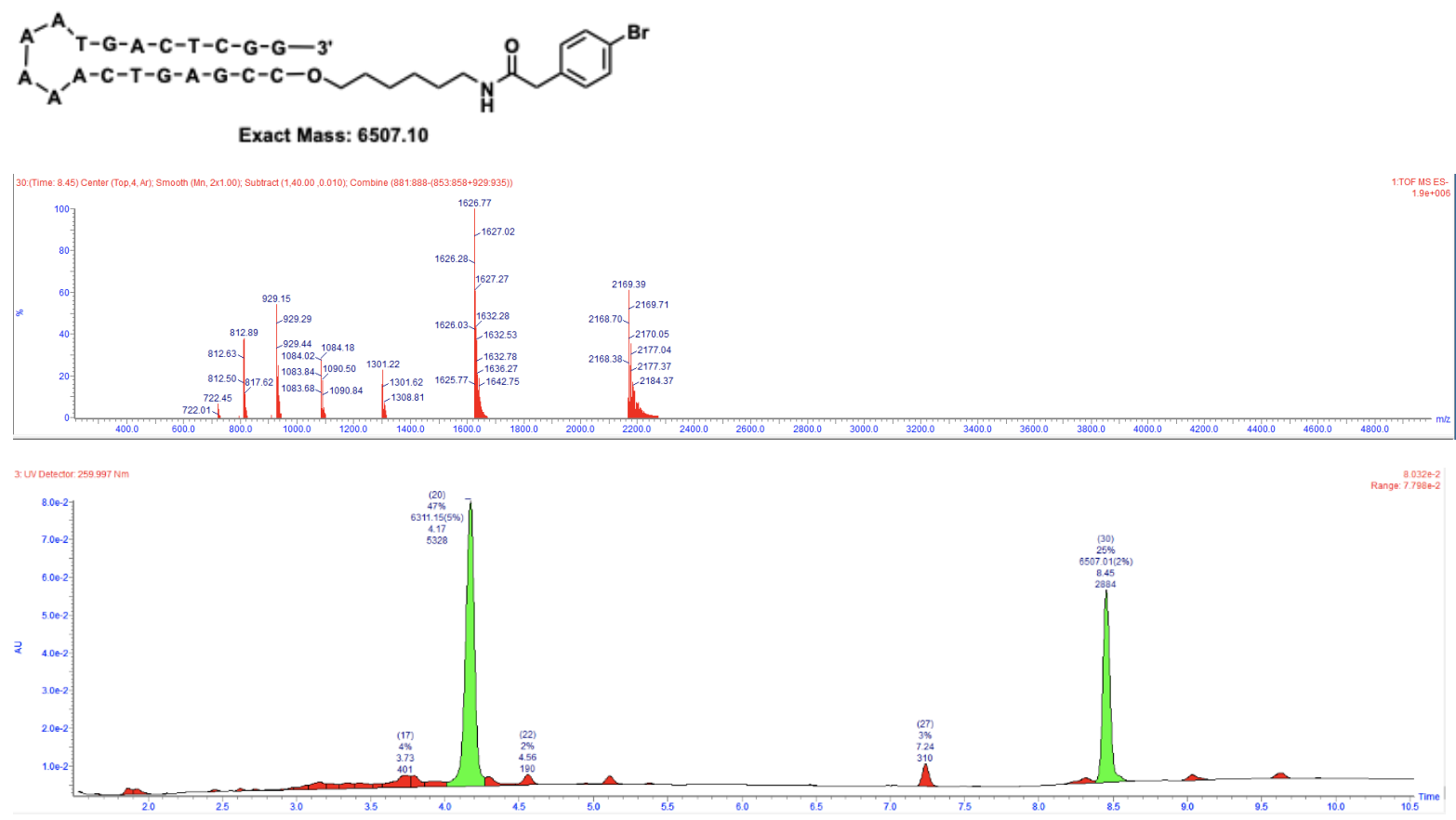
Entry 5: ACN

\section{LC Trace and Mass of SI-2}

Following General Procedure 3 with SI-1

Yield: $12 \%$

Exact mass: 6507.1

Triply charged mass [M-3]/3, calculated: 2168.03; observed 2168.37
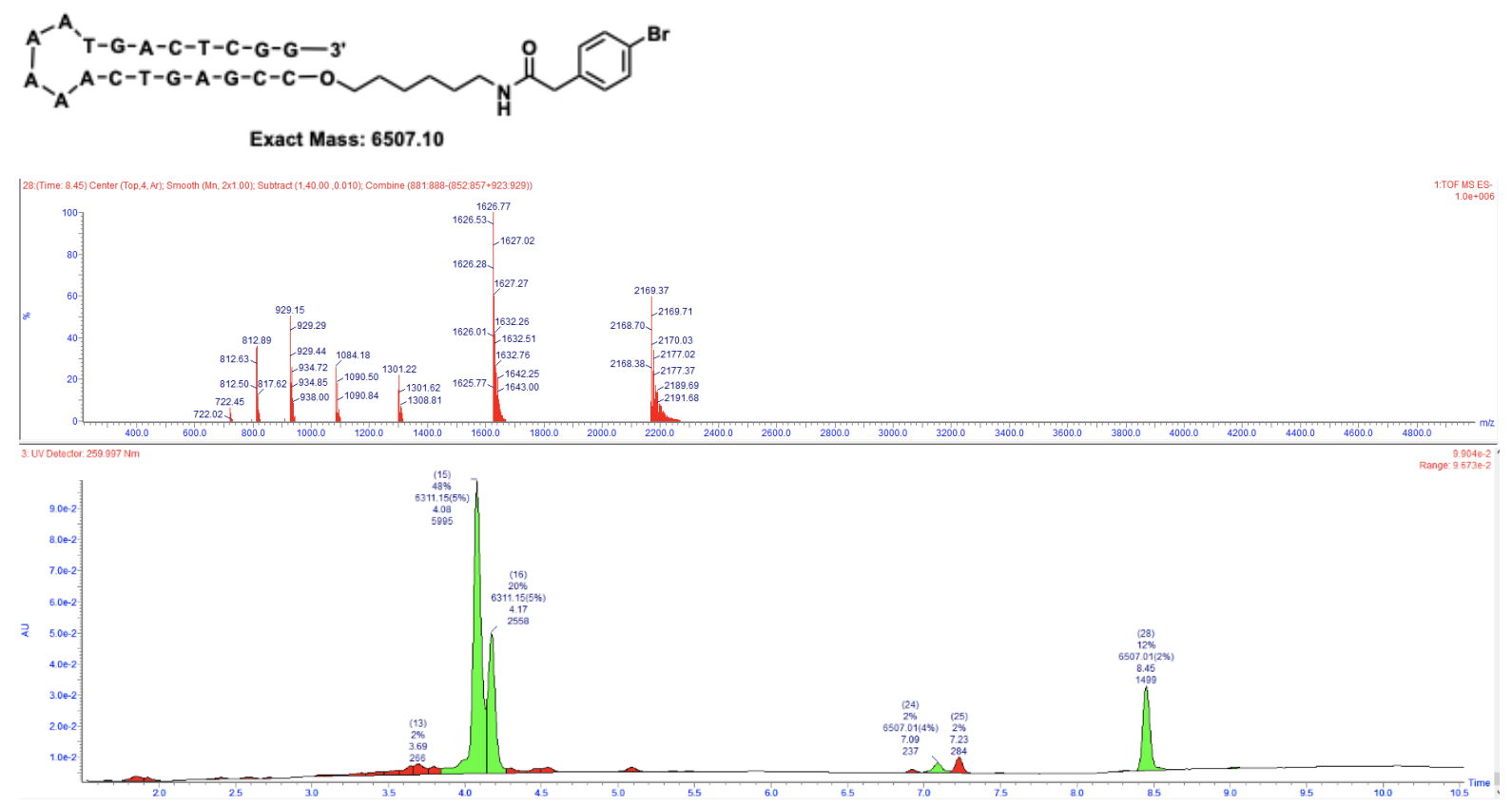
Entry 6: DMA

\section{LC Trace and Mass of SI-2}

Following General Procedure 3 with SI-1

Yield: $48 \%$

Exact mass: 6507.1

Triply charged mass [M-3]/3, calculated: 2168.03; observed 2168.37
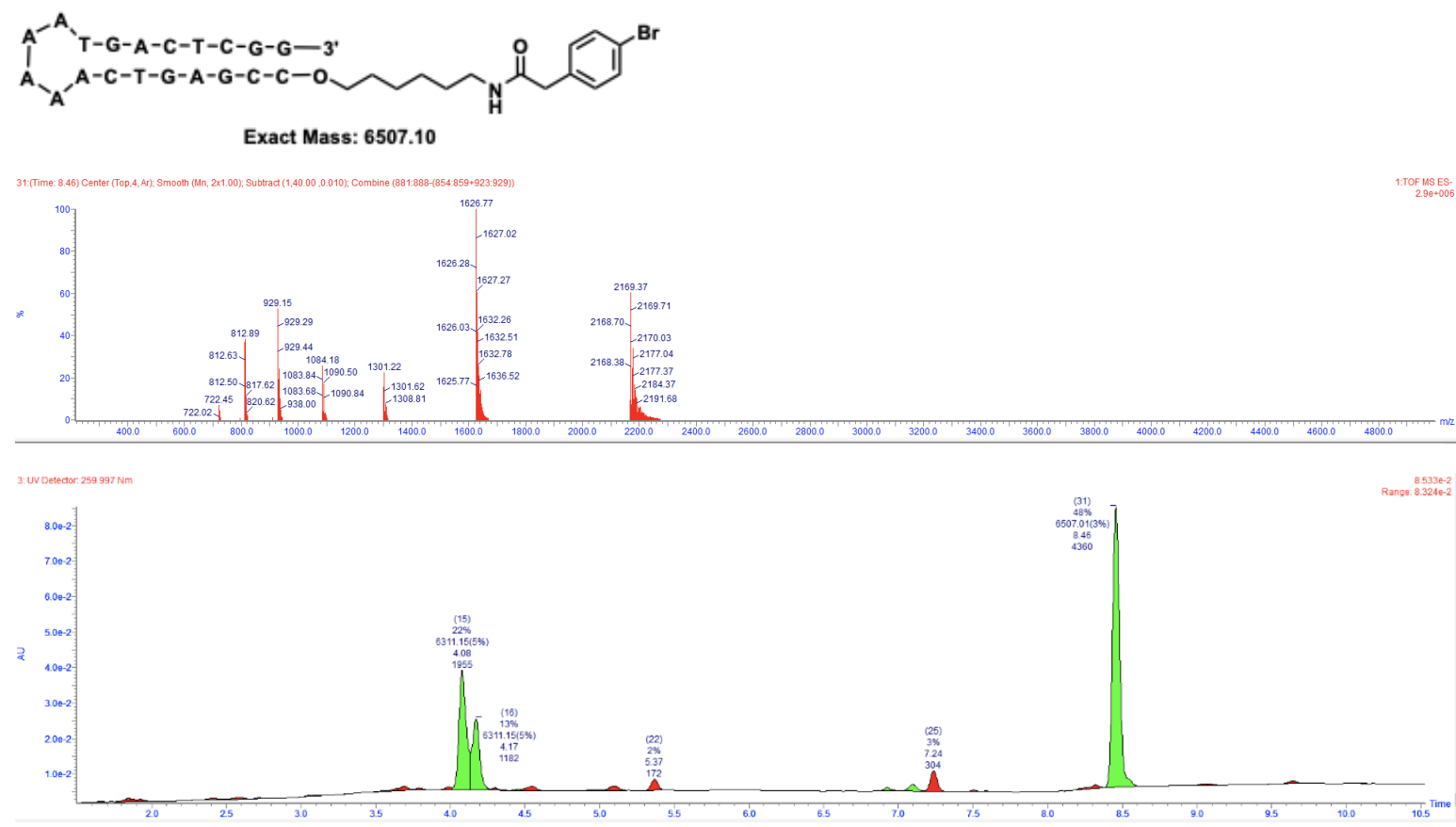


\section{Protocol 3: On-DNA Amide Formation (Free Acid)}

1. Weight out 2.5-5 $\mu \mathrm{mol}$ free acid.

2. Dissolve acid $(2.5-5 \mu \mathrm{mol})$ in $250-500$ DMA to a concentration of $100 \mathrm{mM}$.

3. Add acid solution to the resin bed.

4. Add DIC (1.0 equivalents as compared to acid) by micropipette to a concentration of $100 \mathrm{mM}$

5. Add $\mathrm{NEt}_{3}$ (or DIPEA) (1.5 equivalents as compared to acid) by micropipette to a concentration of $150 \mathrm{mM}$.

6. Vortex reaction mixture and place on orbital shaker (3-6 h) to gently agitate the slurry.

7. Alternatively add small stir bar to the microcentrifuge tube and allow to stir for 3-6h.

Note: Since the DNA linked molecules are at very low concentration, reactants and other reagents should be used at high concentration (100 $\mathrm{mM}$ or higher), rather than as eq to the DNA-conjugate. The relative stoichiometry of the excess reagents is usually still important. 


\section{Trouble Shooting: Frequently Asked Questions (Handling and Simple Reactions)}

What type of centrifuge do I need to spin down my resin?

One does not need to spin the resin slurry very fast to pellet the resin. Any commercially available "Mini Centrifuge" such as the Thomas Scientific Benchmark will do the trick.

How long do I need to spin my samples to separate resin from the eluent?

Length in the centrifuge is solvent dependent. The denser a solvent that the resin slurry is comprised of the longer it will need in the centrifuge. If using very dense halogenated solvents (DCM and HFIP) the resin will float (no centrifugation required) and you can still remove the solvent layer by pipette.

What is the best way to add reagents to a reaction?

We prefer to make stock solutions of reagents and add them to the resin slurry. Dumping in dry solids or pipetting in liquid reagents is also acceptable in most cases.

Shake or stir?

We have found stirring superior in most reactions but shaking works in some cases as well. 


\section{Experimental Procedures and Product Characterization for DNA-bound (Het)Aryl Iodides and Bromides}

Synthesis of DNA-bound (Het)Aryl Iodide and Aryl Bromide

General Procedure 4

$1 \mathrm{M}$ sodium carboxylate solution was made by addition of $1 \mathrm{M} \mathrm{NaOH}$ solution to substrate carboxylic acid. To a $2.0 \mathrm{~mL}$ eppendorf tube was added a stock solution of DNA headpiece $\mathbf{1}\left(20.0 \mu \mathrm{L}, 20 \mathrm{mM}\right.$ in $\mathrm{H}_{2} \mathrm{O}, 400$ nmol), $100 \mathrm{mM}$ borate buffer $(300 \mu \mathrm{L}, \mathrm{pH} 9.5)$, sodium carboxylate solution $\left(40 \mu \mathrm{L}, 1.0 \mathrm{M}\right.$ in $\mathrm{H}_{2} \mathrm{O}, 100$ equiv) followed by DMTMM (40 $\mu \mathrm{L}, 1.0 \mathrm{M}$ in $\mathrm{H}_{2} \mathrm{O}, 100$ equiv). The contents were mixed by vortex and allowed to stand for 2 hours at room temperature. To the reaction solution was added $5 \mathrm{M} \mathrm{NaCl}(40 \mu \mathrm{L})$ followed by cold ethanol $(1.5 \mathrm{~mL})$ and the contents were mixed by vortex and allowed to stand for 20 minutes at $-20{ }^{\circ} \mathrm{C}$. The suspension was then centrifuged at 10,000 RPM for 5 minutes and the supernatant was discarded and trace of EtOH was removed by vaccum. The pellet obtained was then dissolved in $\mathrm{H}_{2} \mathrm{O}$ $(320 \mu \mathrm{L})$ and $\mathrm{CH}_{3} \mathrm{CN}(40 \mu \mathrm{L})$ followed by addition of DIPEA $(40 \mu \mathrm{L})$. The eppendorf was wrapped with Parafilm and aluminum foil and heated in an oil bath at $70{ }^{\circ} \mathrm{C}$ for $2 \mathrm{~h}$. After cooling to room temperature, $5 \mathrm{M} \mathrm{NaCl}(40 \mu \mathrm{L})$ was added followed by cold ethanol $(1.5 \mathrm{~mL})$. The contents were mixed by vortex and allowed to stand for 20 minutes at $-20^{\circ} \mathrm{C}$. The suspension was then centrifuged at 10,000 RPM for 5 minutes and the supernatant was discarded. Trace of $\mathrm{EtOH}$ was removed by vaccum and the pellet obtained was reconstituted in $\mathrm{H}_{2} \mathrm{O}(80 \mu \mathrm{L})$ to give a stock solution of DNA-bound (Het)Aryl at $5 \mathrm{mM}$ concentration.

$0.2 \mu \mathrm{L}$ of the stock solution was diluted with $99.8 \mu \mathrm{L}$ of $\mathrm{H}_{2} \mathrm{O}$ to prepare LCMS sample at $0.01 \mathrm{mM}$ concentration or $2 \mu \mathrm{L}$ of the stock solution was diluted with $98 \mu \mathrm{L}$ of $\mathrm{H}_{2} \mathrm{O}$ to prepare LCMS sample at 0.1 $\mathrm{mM}$ concentration 


\section{LC Trace and Mass of 1}

Following General Procedure 4 with SI-3

Exact mass: 5164.81

Triply charged mass [M-3]/3, caluculated: 1720.60; observed 1720.60
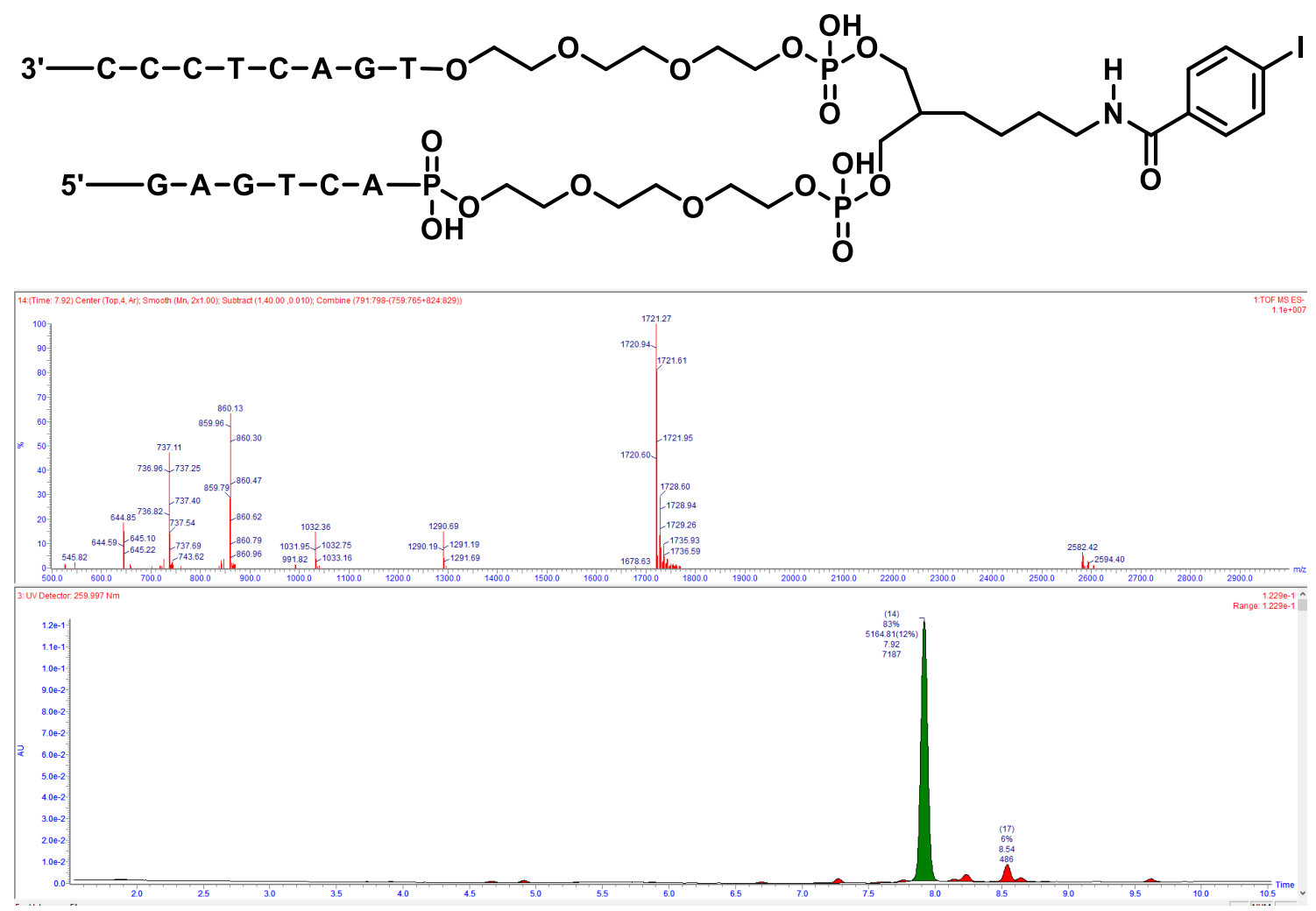


\section{LC Trace and Mass of SI-4}

Following General Procedure 4 with SI-3

Exact mass: 5116.82

Triply charged mass [M-3]/3, caluculated: 1704.61; observed 1704.61
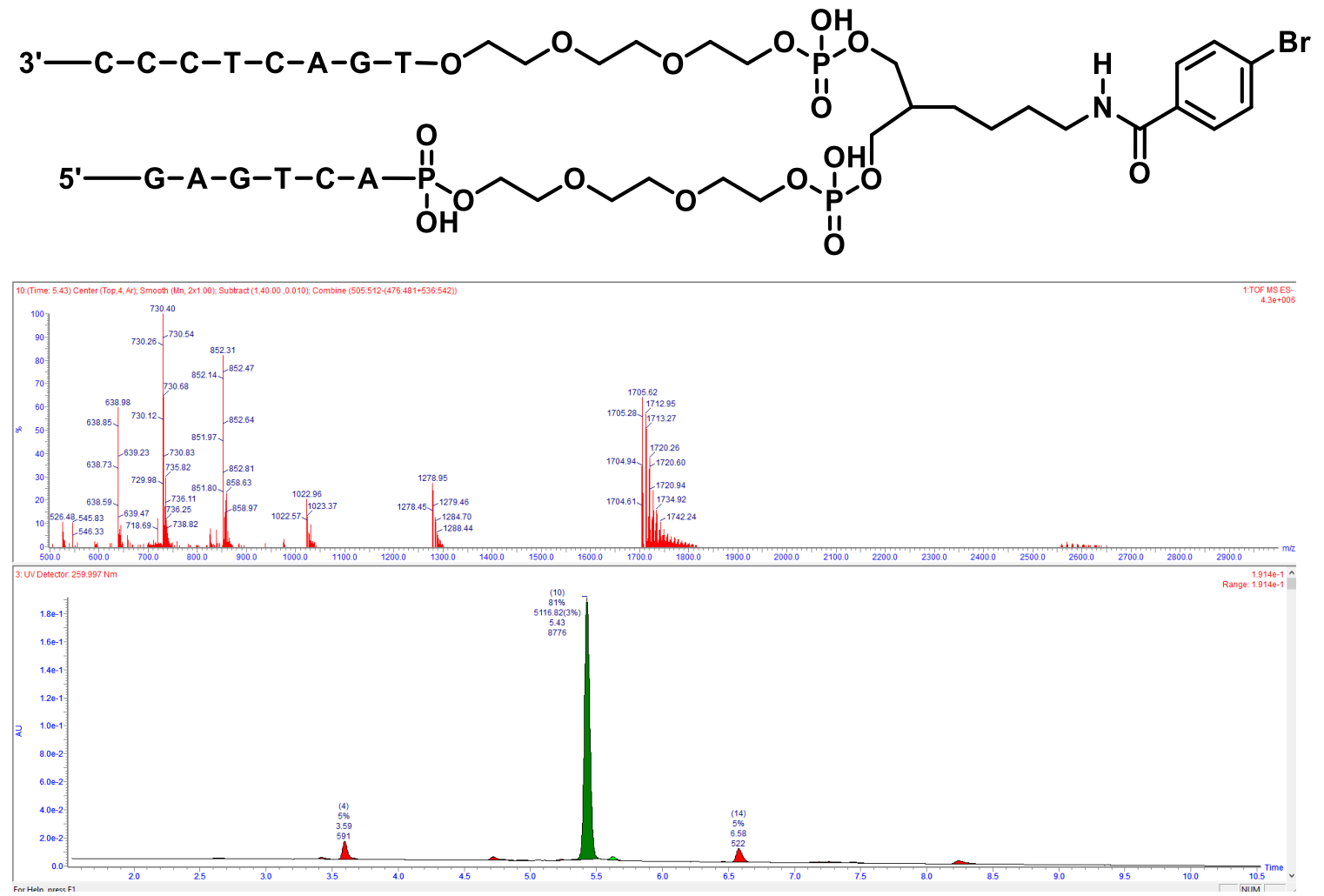


\section{LC Trace and Mass of SI-5}

Following General Procedure 4 with 66

Exact mass: 5411.95

Triply charged mass [M-3]/3, caluculated: 1802.98; observed 1802.99
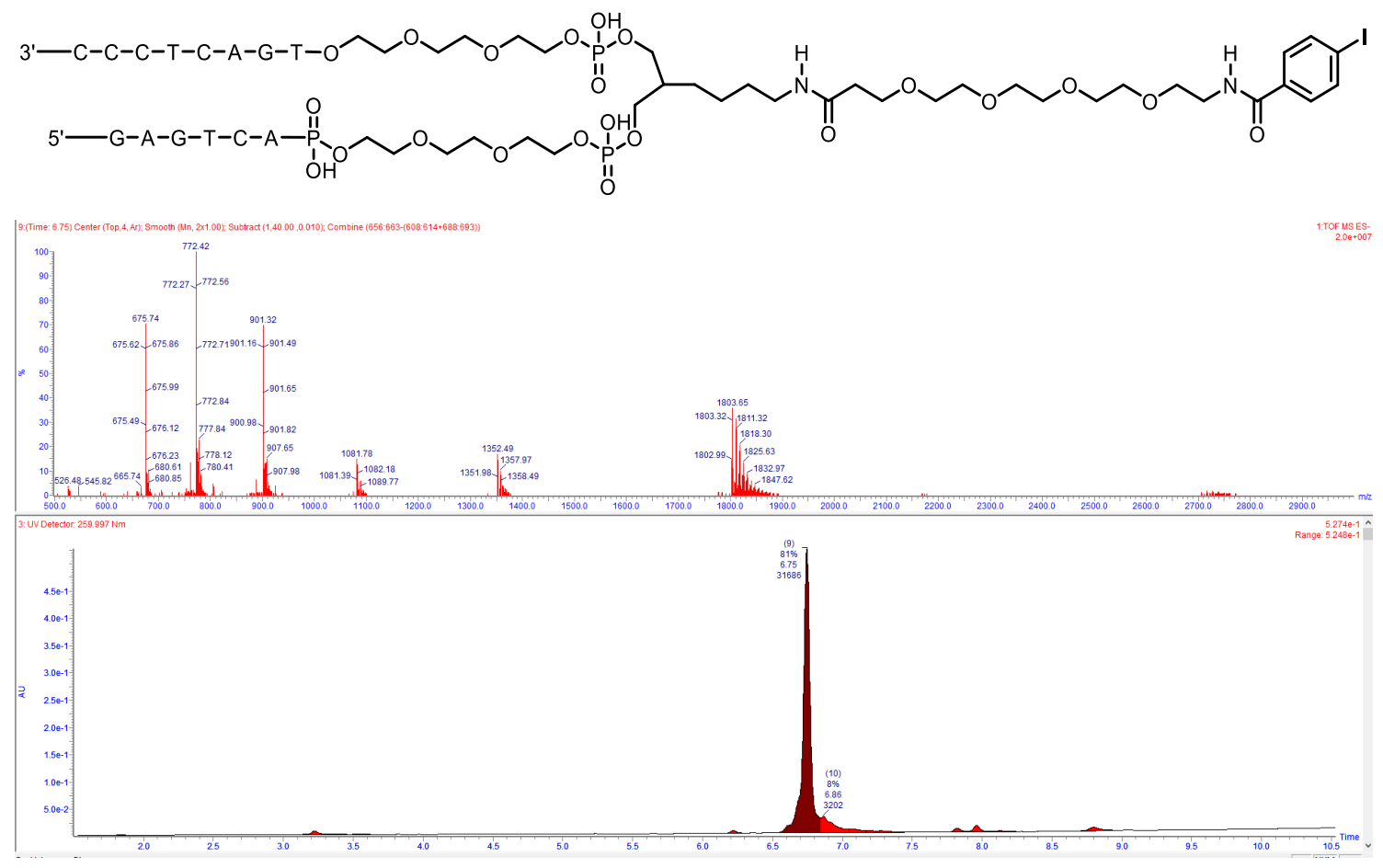


\section{LC Trace and Mass of SI-6}

Following General Procedure 4 with SI3

Exact mass: 5164.81

Triply charged mass [M-3]/3, caluculated: 1720.60; observed 1720.60
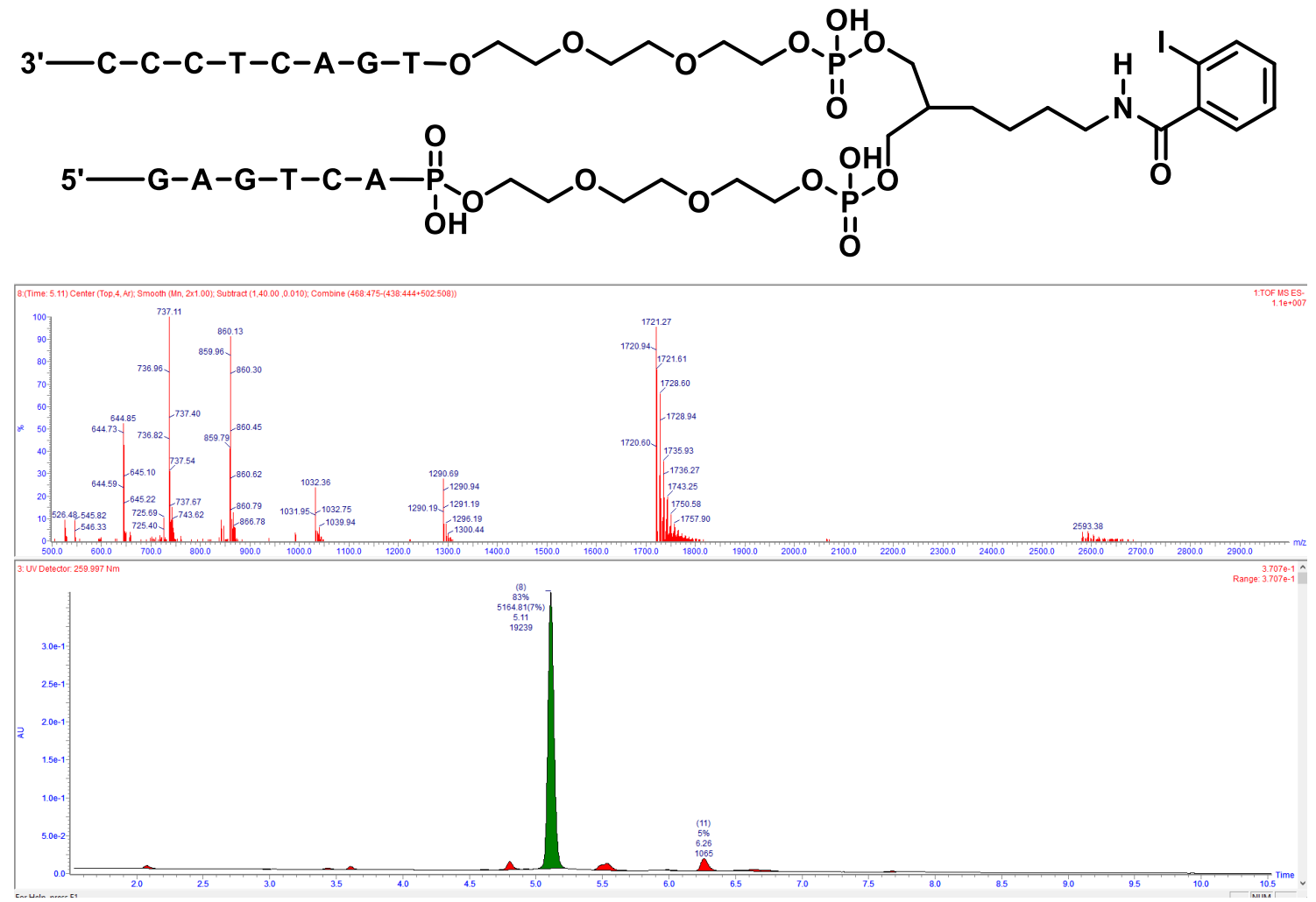
LC Trace and Mass of SI-7

Following General Procedure 4 with SI-3

Exact mass: 5164.81

Triply charged mass [M-3]/3, calculated: 1720.60; observed 1720.60
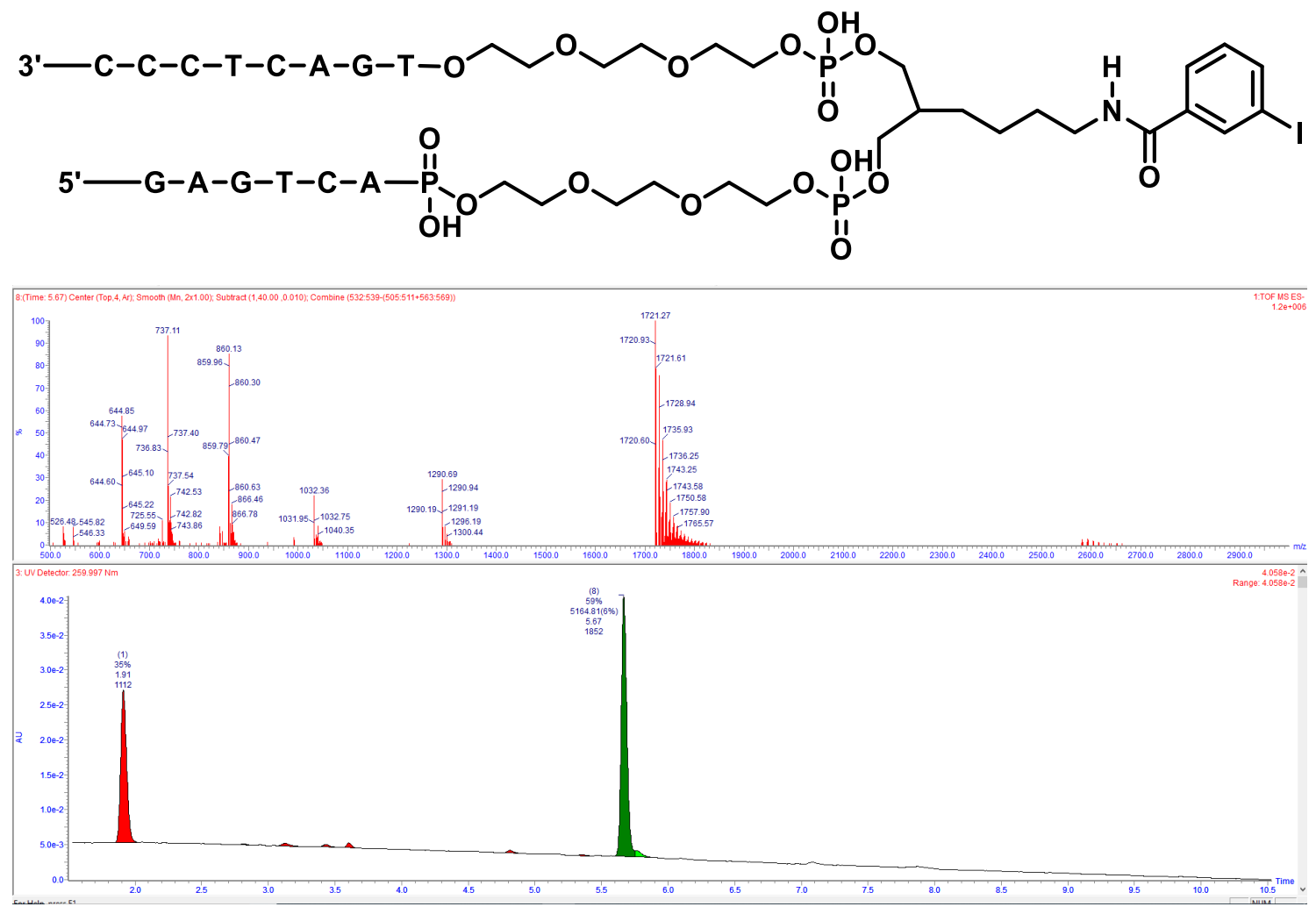

S28 


\section{LC Trace and Mass of SI-8}

Following General Procedure 4 with SI-3

Exact mass: 5178.82

Triply charged mass [M-3]/3, caluculated: 1725.27 ; observed 1725.26
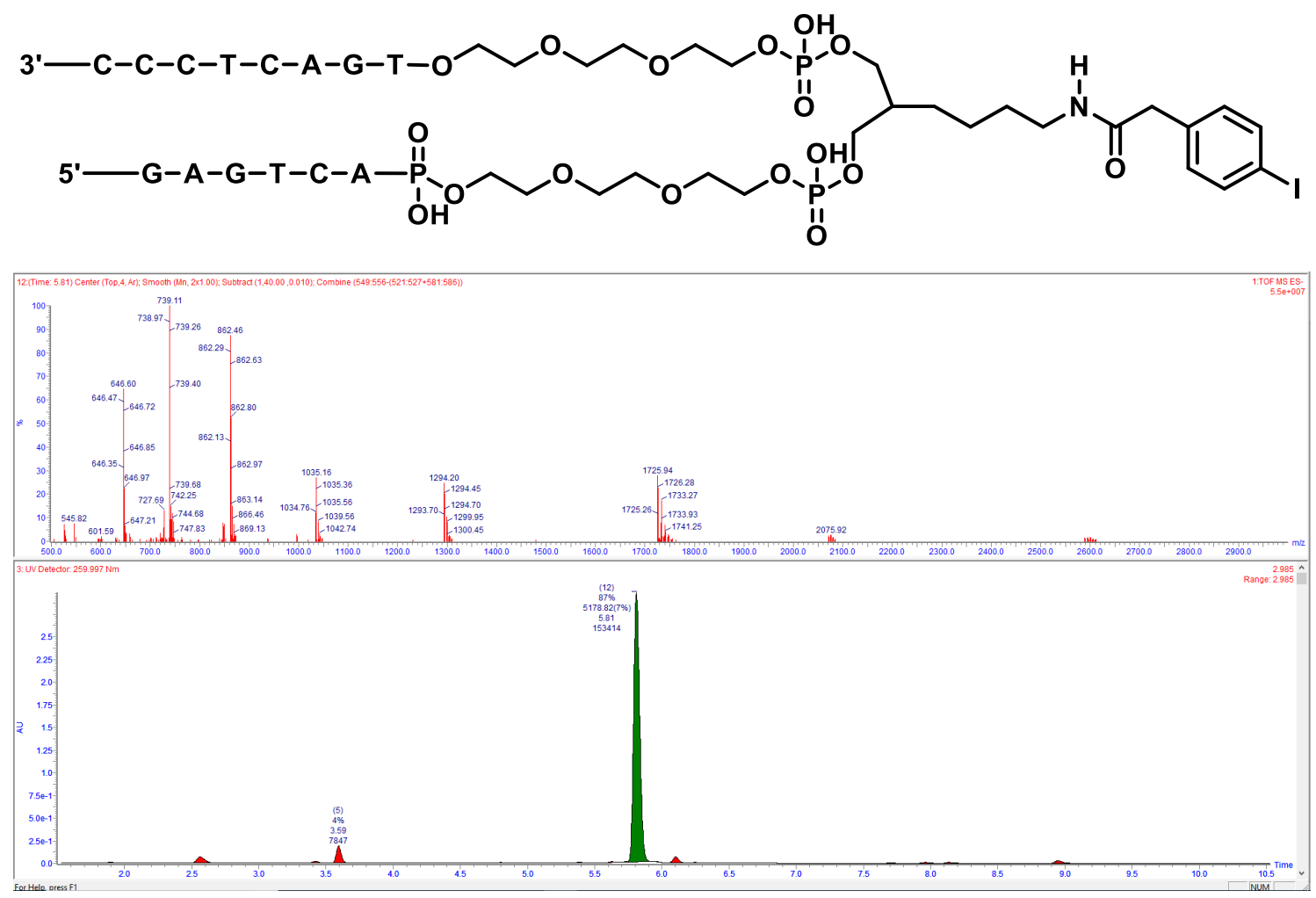


\section{LC Trace and Mass of SI-9}

Following General Procedure 4 with SI-3

Exact mass: 5168.81

Triply charged mass [M-3]/3, caluculated: 1721.94; observed 1721.93
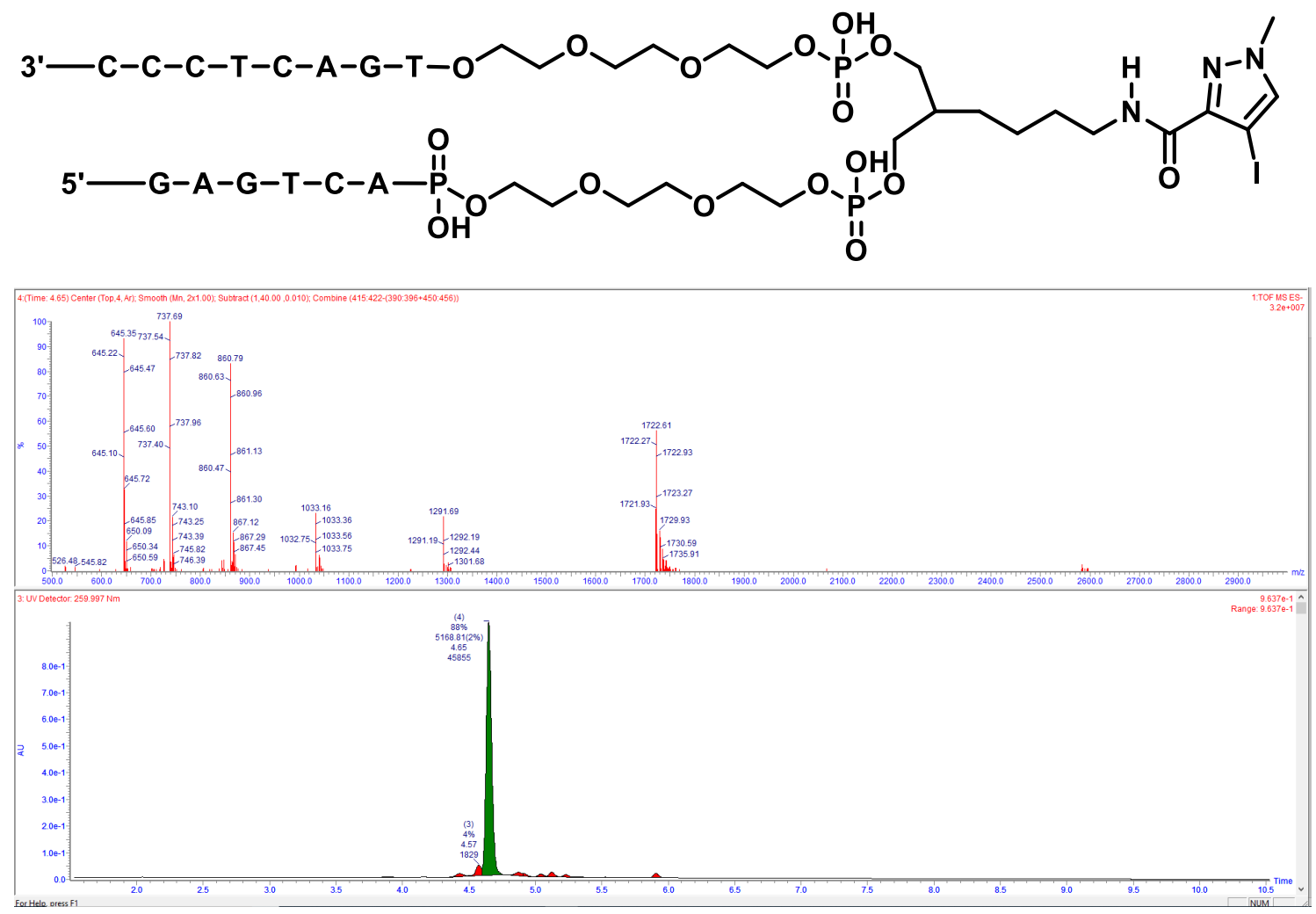


\section{LC Trace and Mass of SI-10}

Following General Procedure 4 with SI-3

Exact mass: 5168.81

Triply charged mass [M-3]/3, caluculated: 1721.94; observed 1721.93
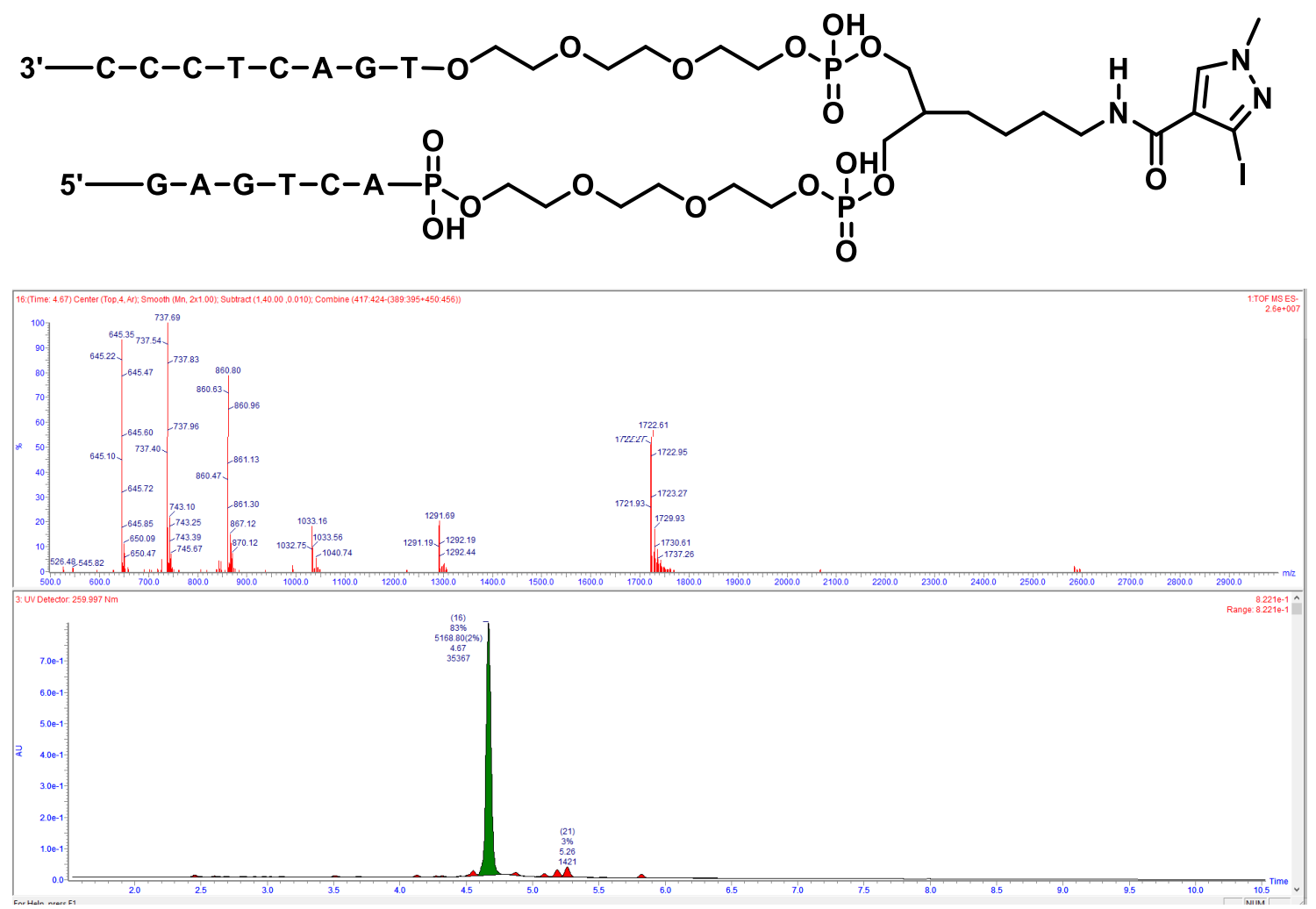
LC Trace and Mass of SI-11

Following General Procedure 4 with SI-3

Exact mass: 5165.80

Triply charged mass [M-3]/3, calculated: 1720.93; observed 1720.93

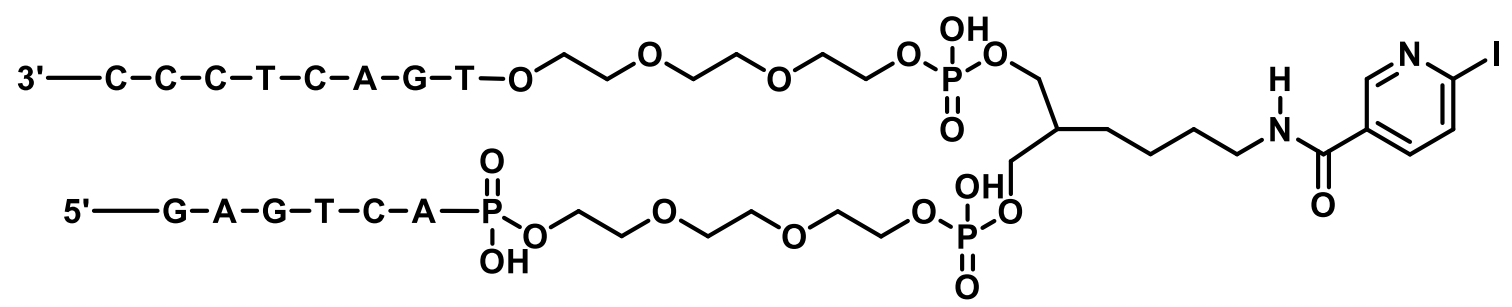

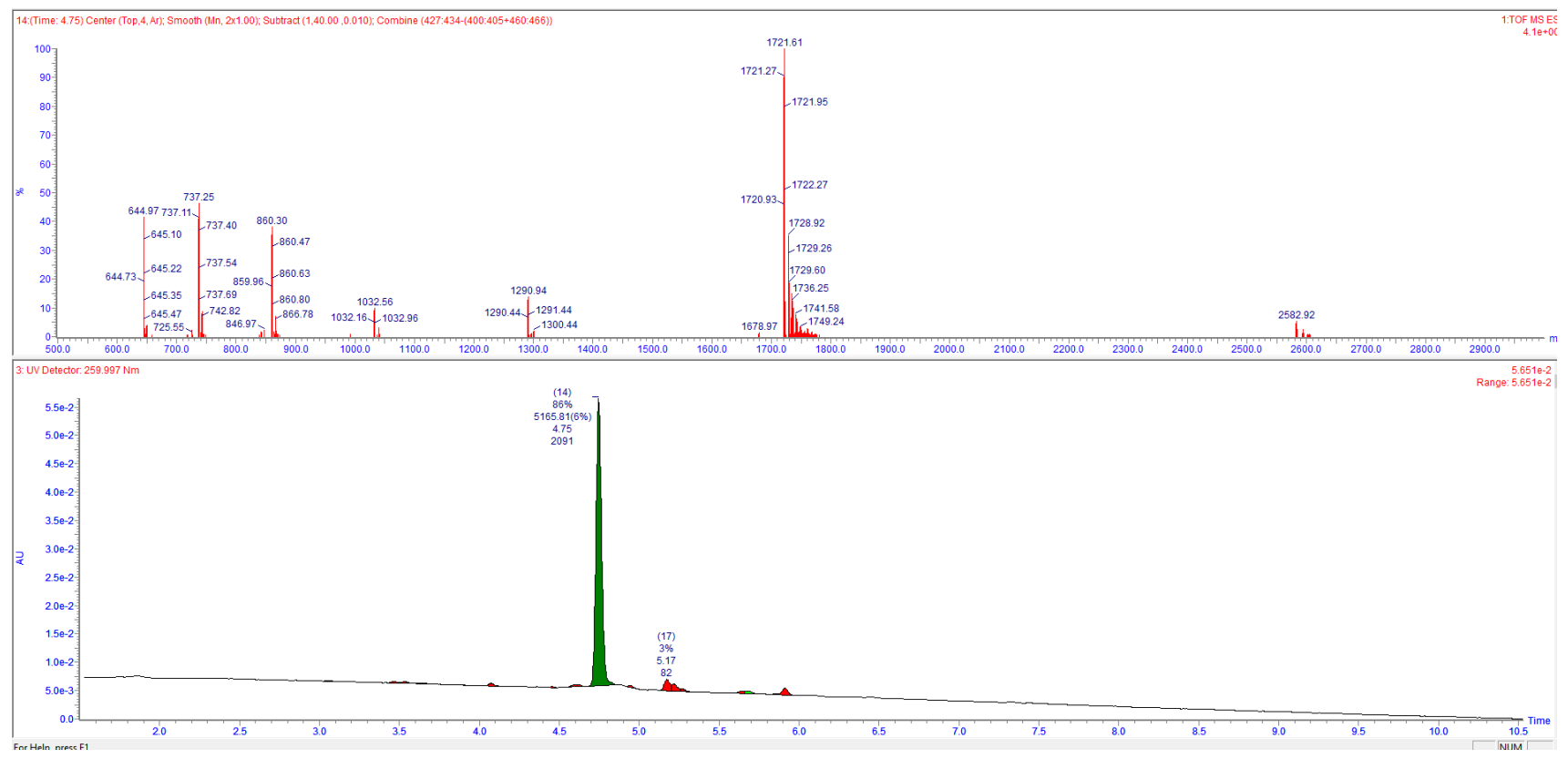

S32 


\section{On-DNA Decarboxylative $\mathrm{sp}^{2}$-sp $\mathrm{sp}^{3}$ Cross Coupling Optimization}

DNA-Ar-I headpiece 1 (10 nmol) was loaded onto resin $(100 \mu \mathrm{L})$ according to general procedure 1 above, and washed with DMA. DMA was degassed by bubbling argon, from a balloon, through it for at least 20 minutes. All solids were weighted into individual Eppindorph tubes. To the solid Ni/ligand mixtures 250 $\mu \mathrm{L}$ DMA was added and the solution was heated at $>70^{\circ} \mathrm{C}$ until the solution was no longer turbid. After cooling, the Ni/ligand was used to dissolve the solid RAE 2.

To the DMA-washed resin bed, a small stir bar, the solid base (by dumping), and the Ni/ligand RAE solution were added. Finally the reductant ( $\mathrm{Zn}$ added as a powder and dumped in, liquid reductants added via micropippett) was added and the tube was capped with a cap that was punctured with a small hole (See Graphical Protocol 5). The reaction vessel was then immediately placed into a large test tube and the Degassing procedure was performed (See General Procedure XX). The reactions were allowed to stir under Argon atmosphere for varying amounts of time. After specified time, the reactions were washed with DMA, eluted (Protocol 5), and analyzed by HPLC-MS. 


\section{Initial Hit}

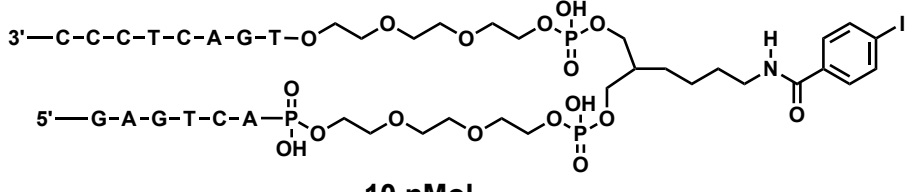

$10 \mathrm{nMol}$

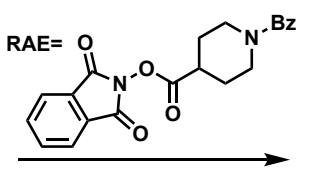

Conditions 250 uL DMA, 18 Hrs, under Ar Atmosphere<smiles>[14CH3]NC(=O)c1ccc(C2CC[NH+](C(=O)c3ccccc3)CC2)cc1</smiles>

\begin{tabular}{|c|c|c|}
\hline Entry & Conditions & Yeild (\%) \\
\hline 1 & 200 mM Zn Powder, 10 mM Ni dtbbpy $\mathrm{Br}_{2}, 100$ mM RAE & 0 \\
\hline 2 & 200 mM Zn Powder, 10 mM NiBr 2 Diglyme, 20 mM dtbbpy, 100 mM RAE & 0 \\
\hline 3 & 200 TDAE, $10 \mathrm{mM}$ Ni dtbbpy $\mathrm{Br}_{2}, 100 \mathrm{mM} \mathrm{RAE}$ & 0 \\
\hline 4 & $300 \mathrm{mM}$ RS, $300 \mathrm{mM} \mathrm{K} \mathrm{CO}_{3}, 10 \mathrm{mM}$ Ni dtbbpy $\mathrm{Br}_{2}, 100 \mathrm{mM}$ RAE & 0 \\
\hline 5 & $300 \mathrm{mM}$ RS, $300 \mathrm{mM} \mathrm{K} \mathrm{CO}_{3}, 10 \mathrm{mM} \mathrm{NiBr}$. Diglyme, $20 \mathrm{mM}$ dtbbpy, $100 \mathrm{mM}$ RAE & 3 \\
\hline 6 & 200 mM Zn Powder, 10 mM Ni dtbbpy $\mathrm{Br}_{2}, 100$ mM RAE, 1:1 DMA:Dioxane & 0 \\
\hline
\end{tabular}

Further Optimization

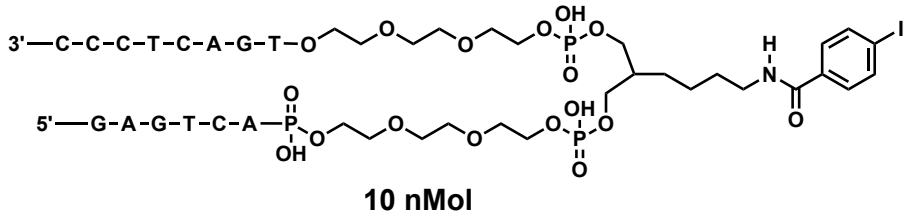

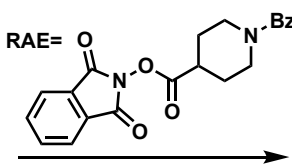

Conditions (Entry 5) 250 uL DMA, 18 Hrs, under Ar Atmosphere<smiles>[14CH3]NC(=O)c1ccc(C2CCN(C(=O)c3ccccc3)CC2)cc1</smiles>

Yeild (\%)

\begin{tabular}{ccc}
\hline Entry & Deviation From Above & Yeild (\%) \\
\hline 7 & $100 \mathrm{mM}[\mathrm{Ni}], 200 \mathrm{mM}$ dtbbpy & 75 \\
8 & $900 \mathrm{mM} \mathrm{RS}, 900 \mathrm{mM} \mathrm{K}_{2} \mathrm{CO}_{3}, 100 \mathrm{mM}[\mathrm{Ni}], 200 \mathrm{mM}$ dtbbpy & 86 \\
9 & $900 \mathrm{mM}$ Silane, $900 \mathrm{mM} \mathrm{K} \mathrm{CO}_{3}$ & 4 \\
10 & $900 \mathrm{mM} \mathrm{RS}, 900 \mathrm{mM} \mathrm{\textrm {K } _ { 2 }} \mathrm{CO}_{3}, 100 \mathrm{mM}$ [Ni], 200 mM dtbbpy, 300 mM RAE & 73 \\
11 & Silane $=\mathrm{DEMS}$ & Trace \\
12 & Silane $=\mathrm{PhSiH}_{3}$ & Trace \\
13 & $+5 \mathrm{mM} \mathrm{Pd(dba)2}$ & 15 \\
14 & $+5 \mathrm{mM} \mathrm{Pd(amphos)2Cl2}$ & 53 \\
15 & $75^{\circ} \mathrm{C}$ & 5 \\
16 & No Resin & N.D. \\
\hline
\end{tabular}



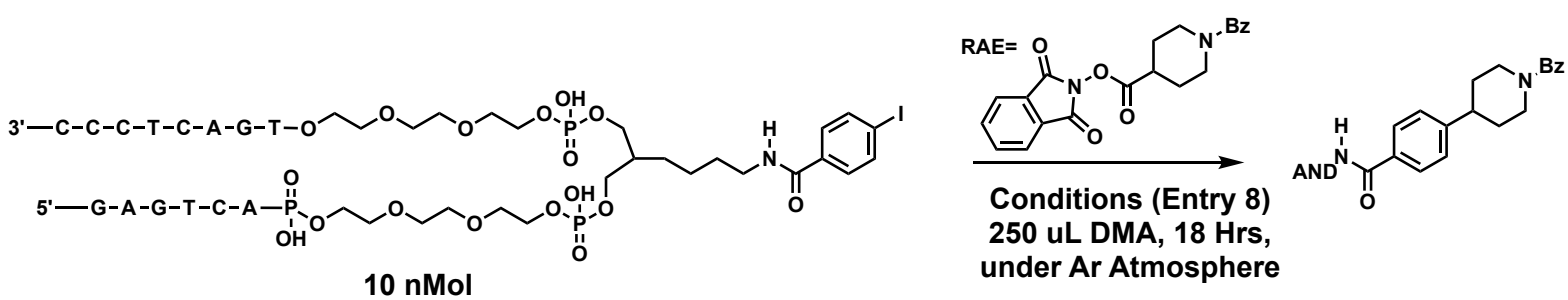

\begin{tabular}{ccc}
\hline Entry & RAE Concentration & Yeild (\%) \\
\hline 17 & $100 \mathrm{mM}$ & 86 \\
18 & $50 \mathrm{mM}$ & 49 \\
19 & $25 \mathrm{mM}$ & 11 \\
20 & $10 \mathrm{mM}$ & 4 \\
\hline
\end{tabular}

\section{Effect of Ni/Ligand Concentration}
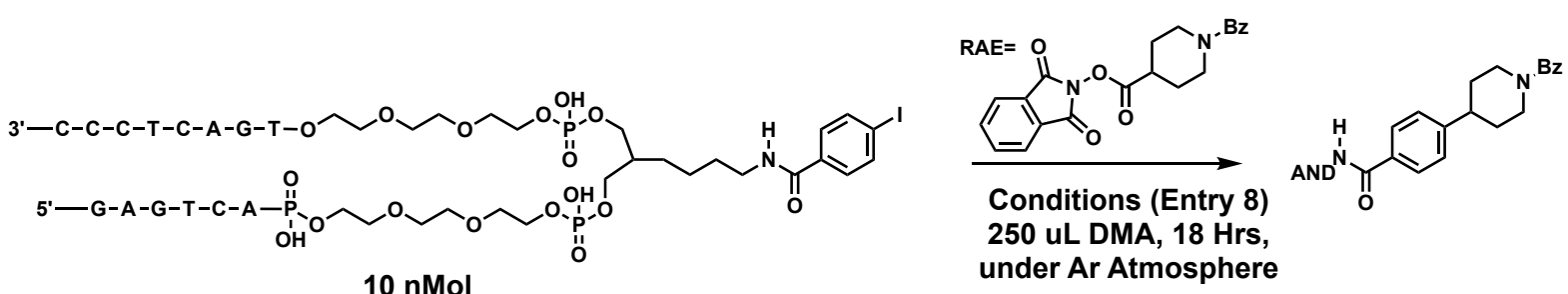

\begin{tabular}{ccc}
\hline Entry & Ni/dtbbpy Concentration & Yeild (\%) \\
\hline 21 & $50 \mathrm{mM} / 100 \mathrm{mM}$ & 63 \\
22 & $25 \mathrm{mM} / 25 \mathrm{mM}$ & 13 \\
\hline
\end{tabular}

Time Course
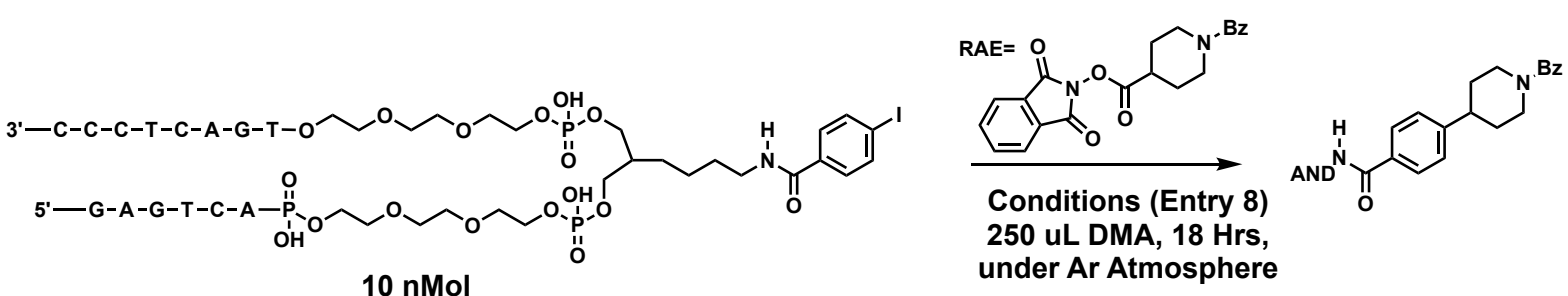

\begin{tabular}{ccc}
\hline Entry & Time (hrs) & Yeild (\%) \\
\hline 23 & 0.5 & 29 \\
24 & 1 & 34 \\
25 & 3 & 39 \\
26 & 6 & 46 \\
\hline
\end{tabular}




\section{Effect of Base}
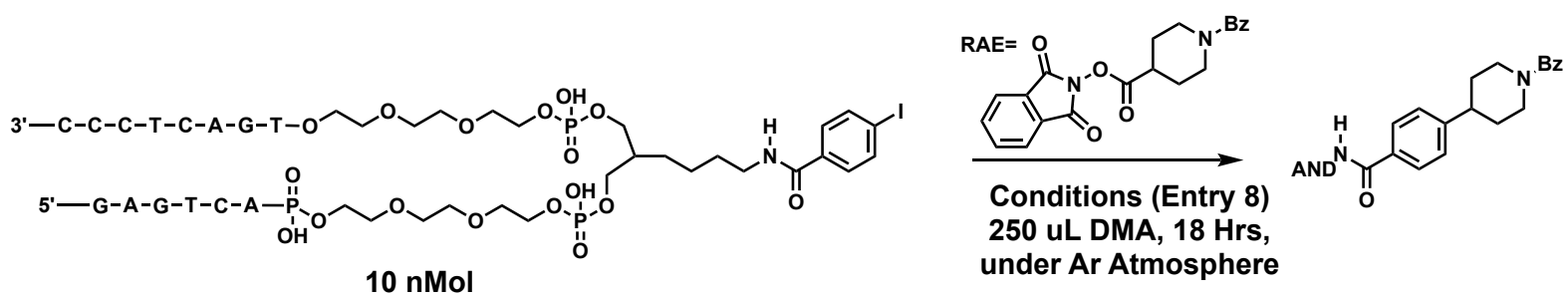

\begin{tabular}{ccc}
\hline Entry & Base & Yeild (\%) \\
\hline 23 & No Base & 8 \\
24 & $\mathrm{Na}_{2} \mathrm{CO}_{3}$ & 34 \\
26 & $\mathrm{Cs}_{2} \mathrm{CO}_{3}$ & 46 \\
\hline
\end{tabular}

Aryl Bromide Optimization

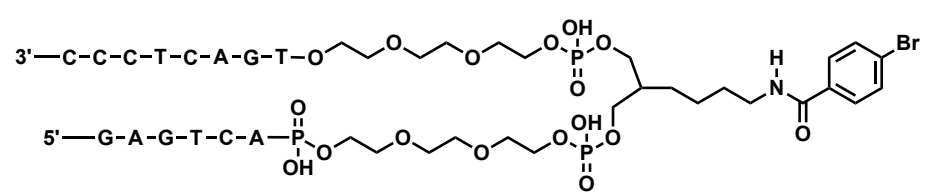

$10 \mathrm{nMol}$

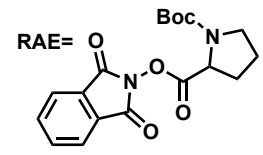

Conditions (Entry 8) 250 uL DMA, 18 Hrs, under Ar Atmosphere [RAE in-situ]

\begin{tabular}{ccr}
\hline Entry & Conditions & Yeild (\%) \\
\hline 27 & $+50 \mathrm{mM} \mathrm{Nal}$ & 11 \\
28 & $+100 \mathrm{mM} \mathrm{Nal}$ & 64 \\
29 & $+250 \mathrm{mM} \mathrm{Nal}$ & 84 \\
30 & $+500 \mathrm{mM} \mathrm{Nal}$ & 66 \\
31 & $+900 \mathrm{mM} \mathrm{Nal}$ & No DNA \\
32 & $60^{\circ} \mathrm{C}$ & No DNA \\
\hline
\end{tabular}




\title{
Protocol 5: On-DNA Decarboxylative $\mathrm{sp}^{2}$-sp $^{3}$ Cross Coupling
}

\author{
Sp2-Sp3 Cross Coupling- in situ activation
}

1. Degas DMA by bubbling Ar for 15 minutes.

2. RAE In Situ: Weigh out acid $(0.05 \mathrm{mmol})$ and N-Hydroxypthalimide (NHPI), into a 1.5 $\mathrm{mL}$ microcentrifuge tube and dissolve in $100 \mu \mathrm{L}$ Degassed DMA to create a $500 \mathrm{mM}$ RAEactivation solution.

a. Add DIC $(0.05 \mathrm{mmol}, 7.9 \mu \mathrm{L})$ to the NHPI/Acid mixture, vortex and allow to sit for at least 1 hour.

3. Isolated RAE: Weight out RAE (0.025 mmole) and dissolve in $50 \mu \mathrm{L}$ DMA.

4. Create Ni stock (125 mM NiBr 2 Diglyme, $250 \mathrm{mM}$ dtbbpy) $3 \mathrm{~mL}$.

a. Weight $132 \mathrm{mg} \mathrm{NiBr} 2$ Diglyme and $201 \mathrm{mg}$ dtbbpy into 1 dram vial. Add $3 \mathrm{~mL}$ degassed DMA and heat while stirring between 70-100 C until the solution is not turbid. Allow to cool to room temperature.

5. To the reaction vessel ( $2 \mathrm{~mL}$ microcenterfuge) with prepared DNA/resin slurry, add a small stir bar, $\mathrm{K}_{2} \mathrm{CO}_{3}(31 \mathrm{mg}), \mathrm{Ni} / \mathrm{Ligand}$ Stock $(200 \mu \mathrm{L})$, RAE solution $(50 \mu \mathrm{L})$, and $\mathrm{NaI}(9 \mathrm{mg})$ if using Ar-Br..

6. Add Rubin's Silane, $\left(\mathrm{Ph}(\mathrm{O}-\mathrm{iPr}) \mathrm{SiH}_{2}, 42 \mu \mathrm{L}\right.$, commercially available from Sigma) and put a cap on the reaction vial with a small hole in it and immediately perform degassing procedure.

7. Degassing procedure:

a. Place microcentrifuge tube into large test tube and cap with septum.

b. Evacuate atmosphere under high vac for 30 seconds.

c. Backfill with argon from balloon.

d. Repeat 3-5 times. Remove balloon and vacuum line and parafilm the septum and reaction vessel leaving the reaction tubes inside the larger test tube.

e. Degassing procedure adapted from Wang, et al. PNAS, 2018

8. Allow to stir ( $>500 \mathrm{rpm})$ at room temperature for 18-24 hours.

a. Upon mixing the slurry should turn black. It may or may not turn white/beige after 24 hours (Protocol 5A)

b. Alternatively, remove reactions from tubes after $1.5 \mathrm{hrs}$. Spin them down and decant reaction solution. Add new reaction solution $(200 \mu \mathrm{L} \mathrm{Ni} /$ dtbbpy stock, $50 \mu \mathrm{L}$ RAE stock, $42 \mu \mathrm{L}$ silane) and perform degassing procedure. Allow reaction to stir for another 1.5 hours. (Protocol 5B) 
Note: For this reaction the best DNA recovery is achieved when the EtOH ppt is done using $5 \mathrm{~mL}$ of cold EtOH (in a $6 \mathrm{~mL}$ tube) instead of $2 \mathrm{~mL}$. 


\section{Graphical Supporting Information: On-DNA Decarboxylative $\mathbf{s p}^{2}-\mathrm{sp}^{3}$ Cross Coupling}

\section{(Protocol 5)}

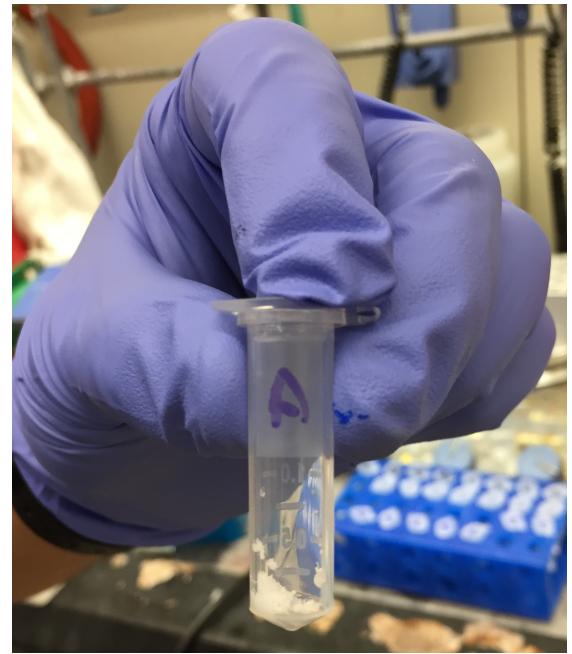

$2 \mathrm{~mL}$ Tube with DNA/Resin slurry, $\mathrm{K}_{2} \mathrm{CO}_{3}(31 \mathrm{mg})$, and small stir bar.

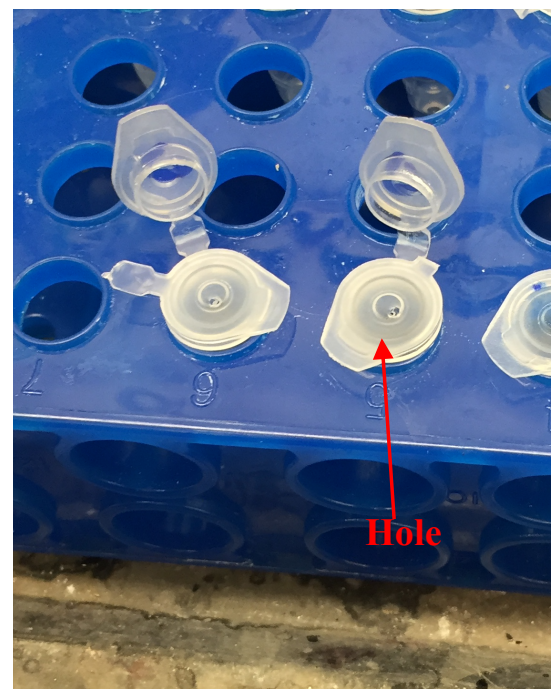

Tubes capped with a cap with a small hole.

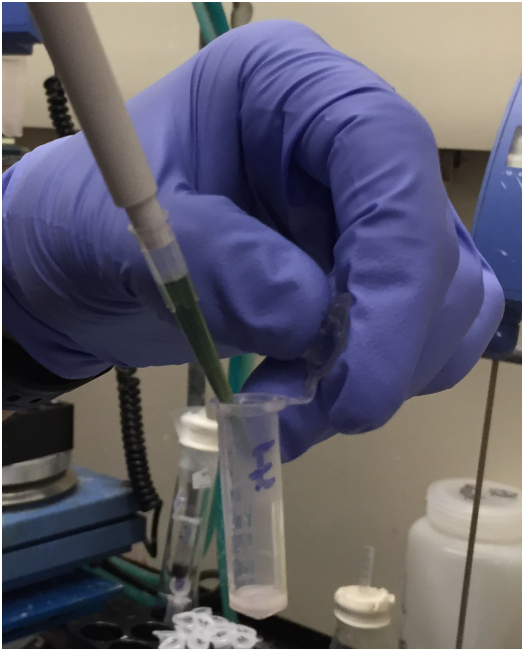

Addition of Ni/dtbbpy (125 $\mathrm{mM} / 250 \mathrm{mM})$ stock $(200 \mu \mathrm{L})$.

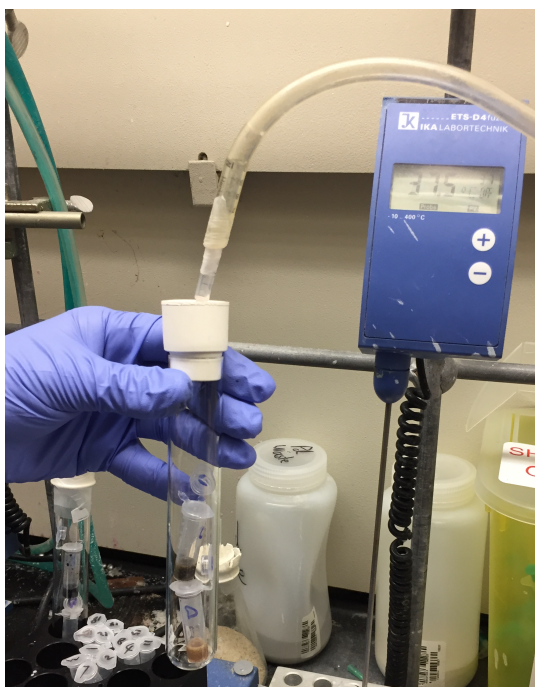

Reaction vessels placed in test tube. Atmosphere evacuated.

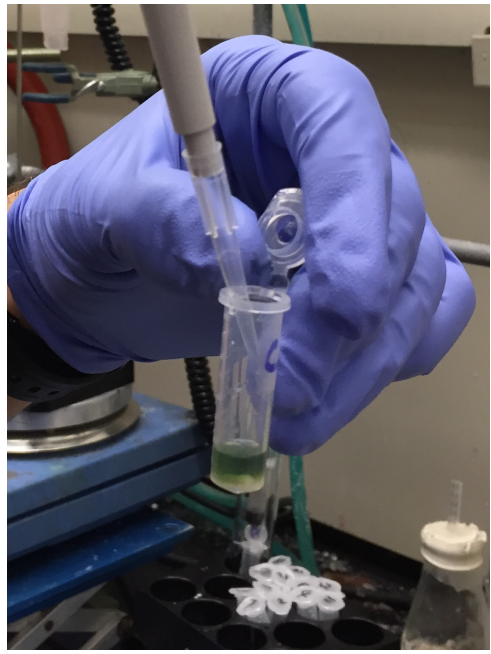

Addition of in situ RAE (500 $\mathrm{mM}$ ) formation solution (50 $\mu \mathrm{L})$.

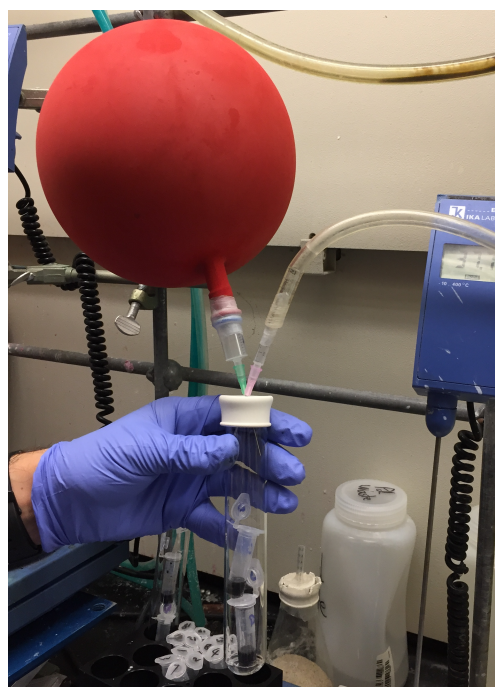

Tube backfilled with argon from balloon. 


\section{Trouble Shooting: Frequently Asked Questions (On-DNA Decarboxylative sp²- $\mathbf{s p}^{3}$ Cross Coupling Reaction)}

Is the source of ligand and nickel important for this reaction?

We prefer to purchase the $\mathrm{NiBr}_{2}$ Diglyme (Lot \# SHBJ3080) from Sigma Aldrich and the dtbbpy from combi-blocks. Interestingly, we started our studies with a bottle of nickel that had been open for a few weeks. Upon switching to a new bottle of nickel (Lot \# SHBK0798) we noticed remarkably decreased yields. We believe that this could be due to either the hydration state of the nickel or the batch quality.

How important is the degassing procedure?

Although not explicitly tested during our study, previous reports maintain that strict elimination of dissolved oxygen is imperative for the success of the reaction. Running the reaction in a glove box will eliminate the degassing procedure.

How important is reaction time?

Most of the substrates required 18-24 hours to reach completion while allowing the reaction to run for more significantly more than 24 hours did not improve yields and resulted in decreased DNA recovery.

Can reaction color be used as an indicator?

In general if the reaction turns black upon the addition of silane it will work. If it changes to any other color it usually meant that something went wrong and the reaction will not be successful. Most reactions stay black during the course of the reaction while some will slowly change from black to beige.

How can I increase my DNA recovery?

Increasing the amount of EtOH:elute buffer during EtOH ppt step will markedly increase the recovered DNA. 


\section{Reaction Products of on-DNA Decarboxylative sp2-sp3 Coupling Reaction}

\section{Products}

\section{LC Trace and Mass of 3}

Following General Procedure 5A with 1

Yield: $86 \%$

Exact mass: 5226.01

Triply charged mass [M-3]/3, caluculated: 1741.00; observed 1741.00
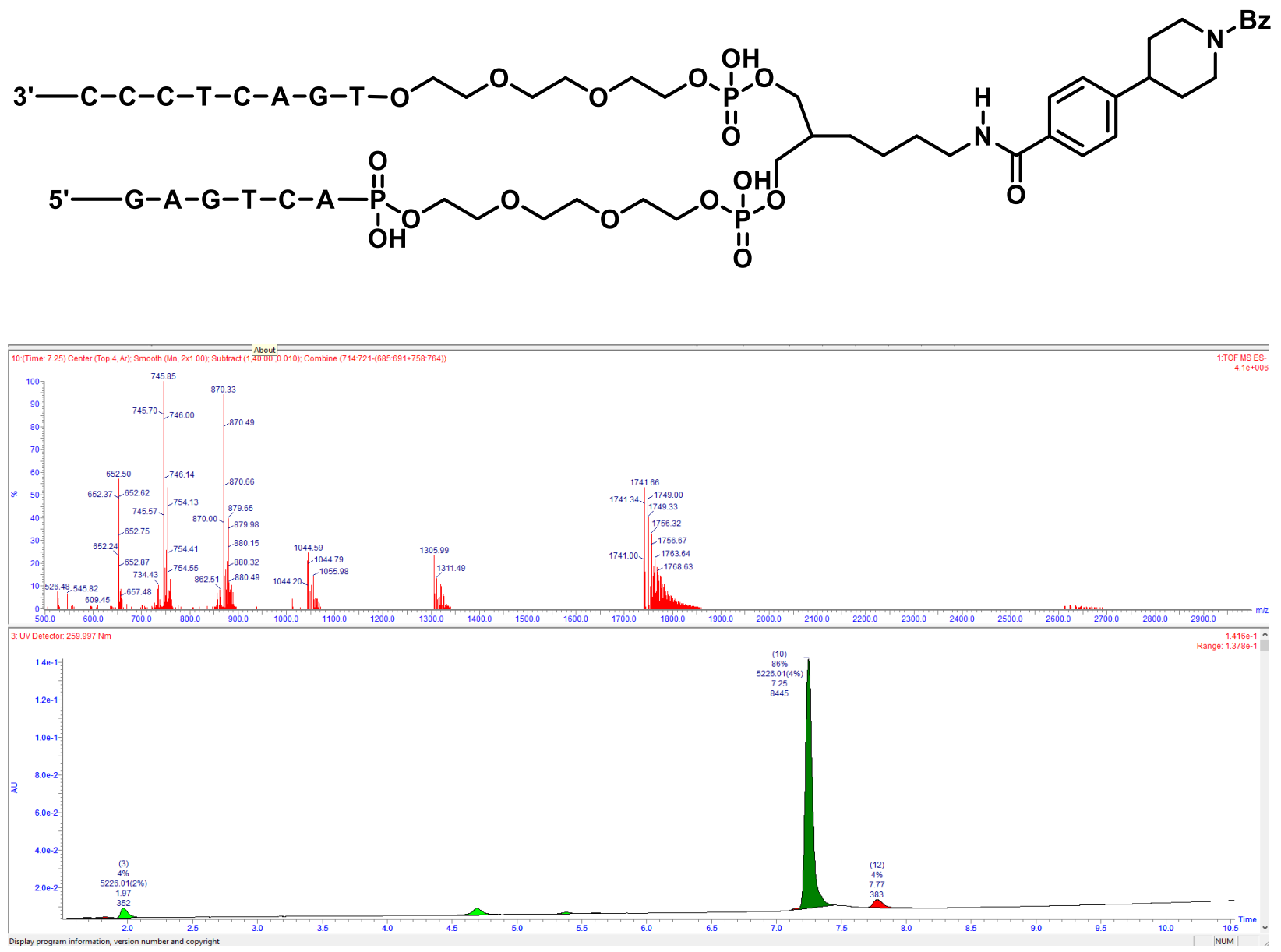


\section{LC Trace and Mass of 3 in tripliacate}

Following General Procedure 5B with 1

Yields: $82 \%, 75 \%, 76 \%$

Average Yield: $77.67 \%$

SDs: 3.7

Exact masses: 5226.01

Triply charged masses [M-3]/3, caluculated: 1741.00; observed 1741.00, 1741.00, 1741.00
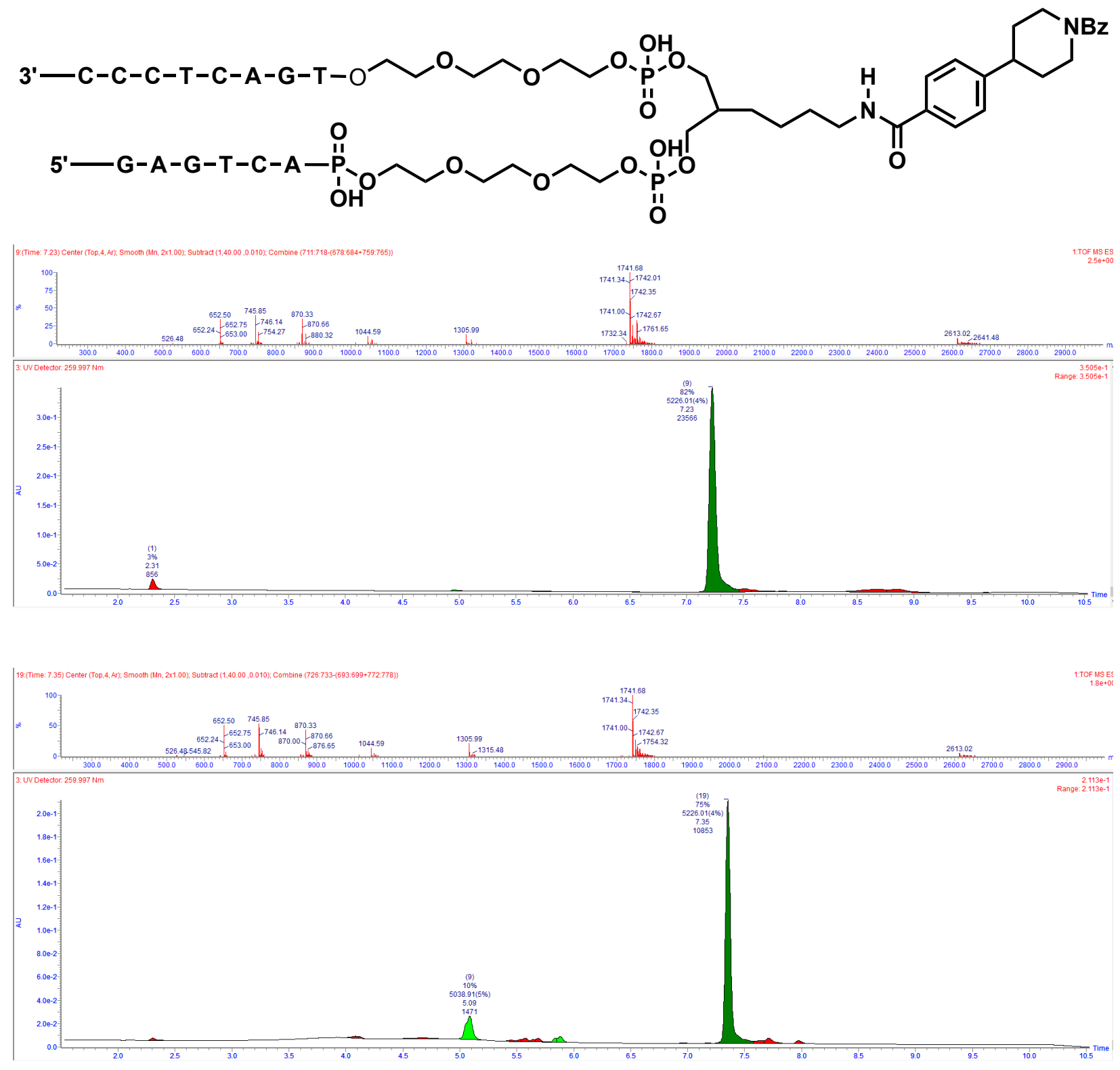


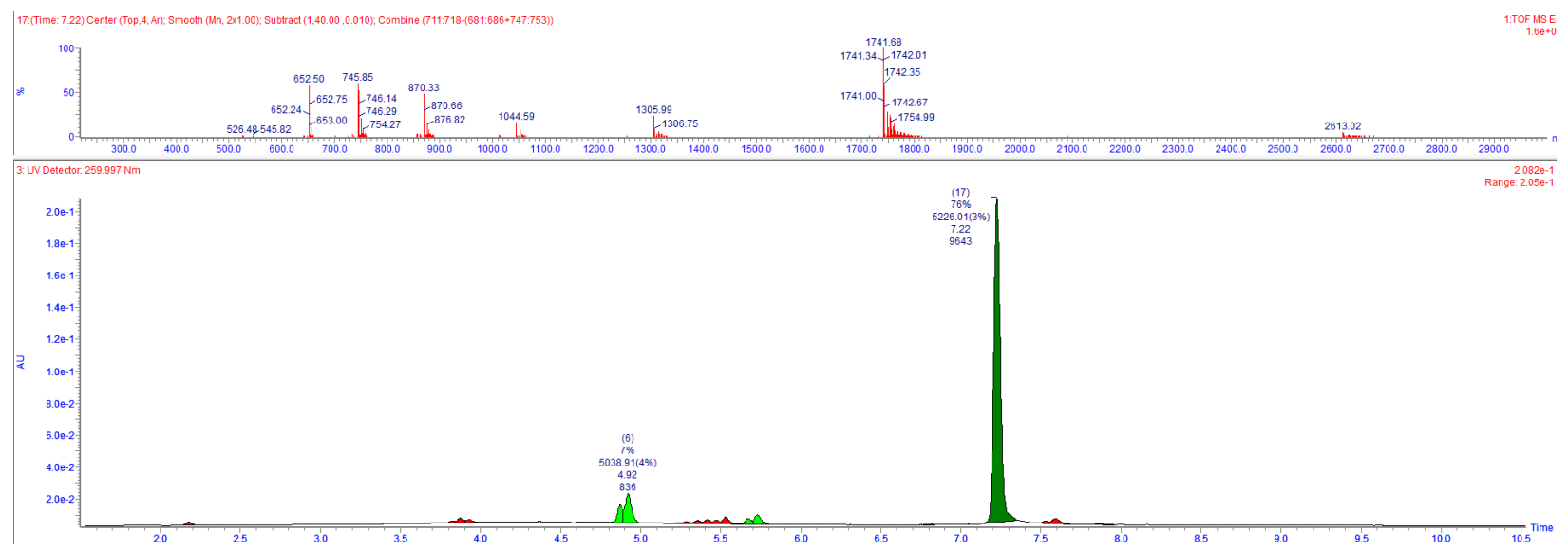




\section{LC Trace and Mass of 4}

Following General Procedure 5A with 1

Analysis method: $\mathbf{X X}$

Yield: $77 \%$

Exact mass: 5222.03

Triply charged mass [M-3]/3, caluculated: 1739.68; observed 1739.68
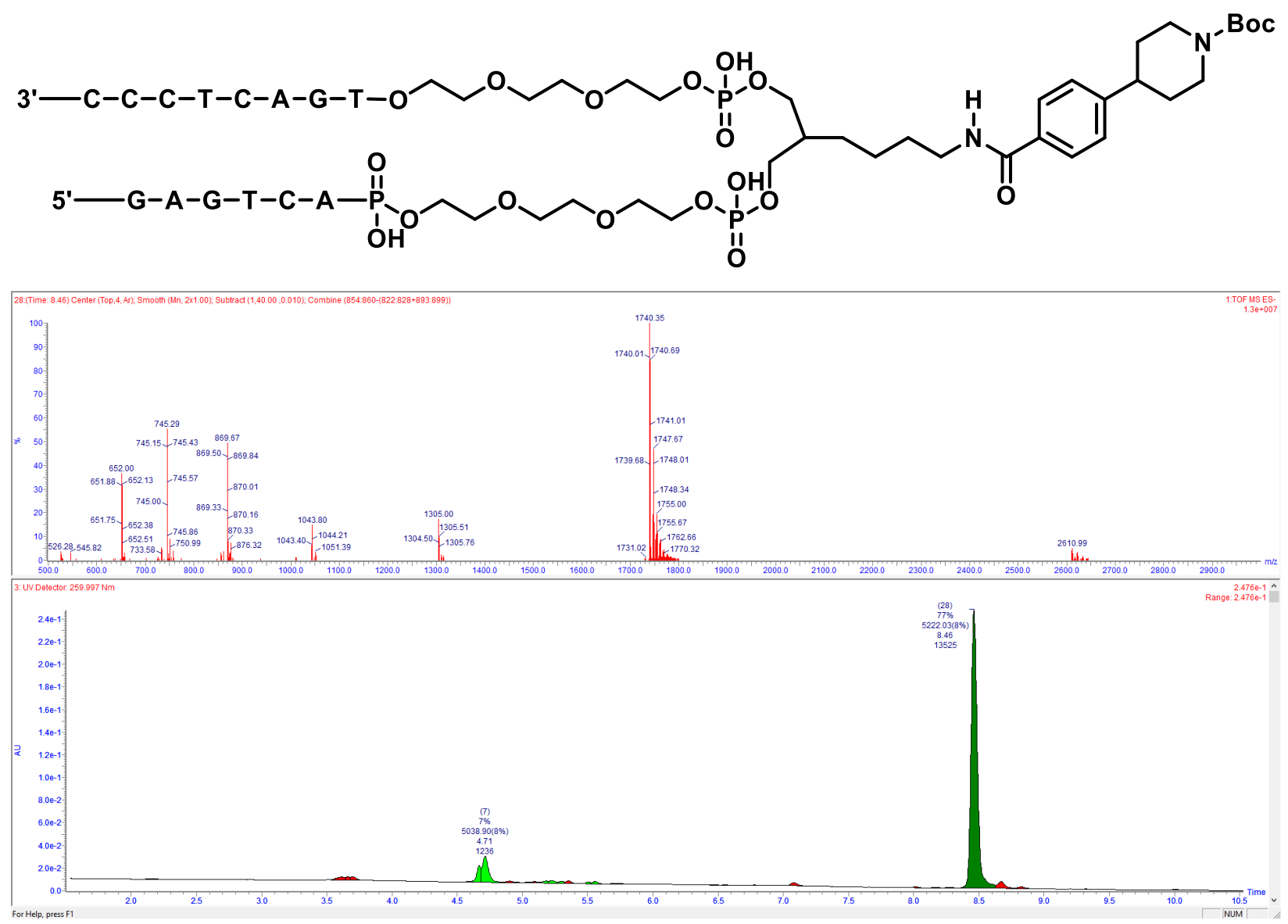


\section{LC Trace and Mass of 4 in tripliacate}

Following General Procedure 5B with 1

Yields: $62 \%, 69 \%, 70 \%$

Average Yield: 67\%

SDs: 4.35

Exact masses: 5222.03

Triply charged masses [M-3]/3, caluculated: 1739.68; observed 1739.68,

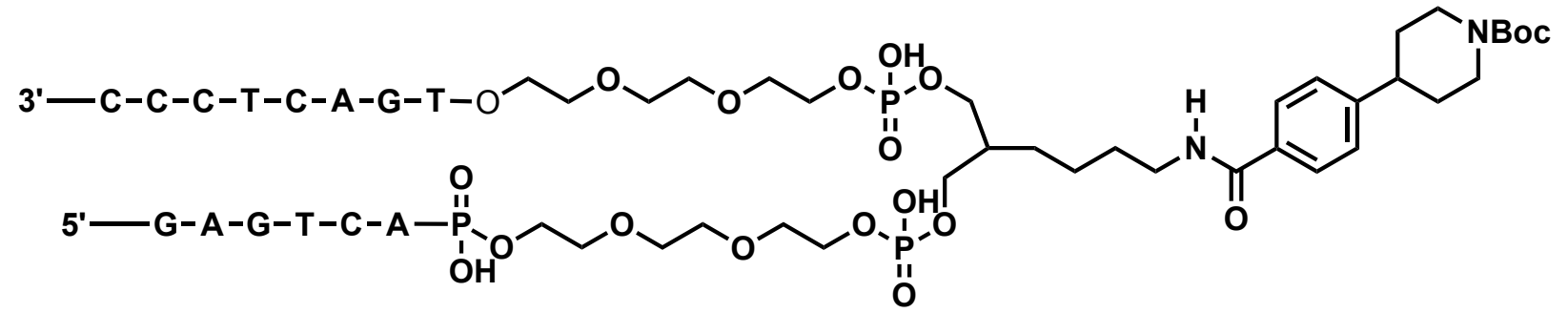
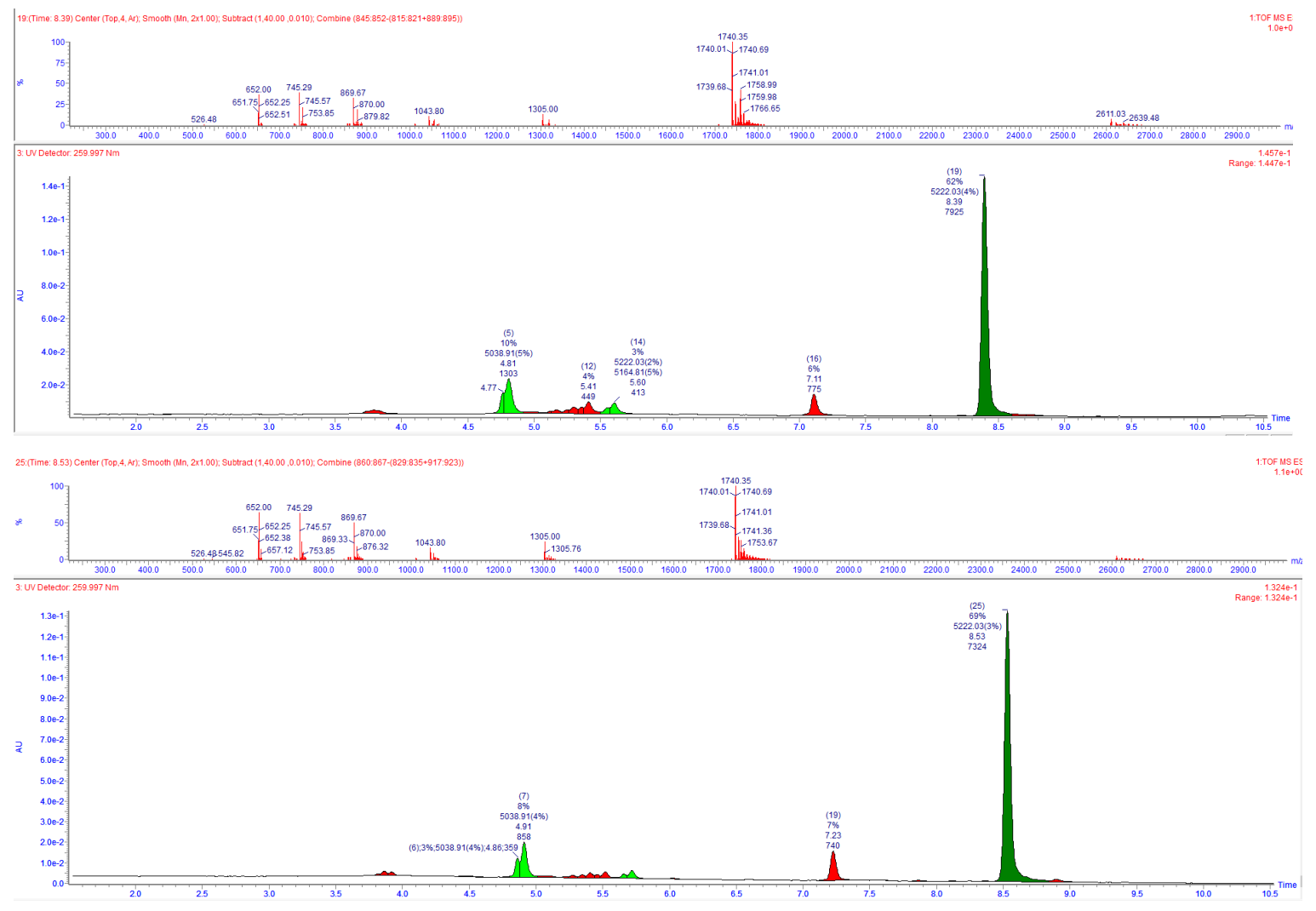


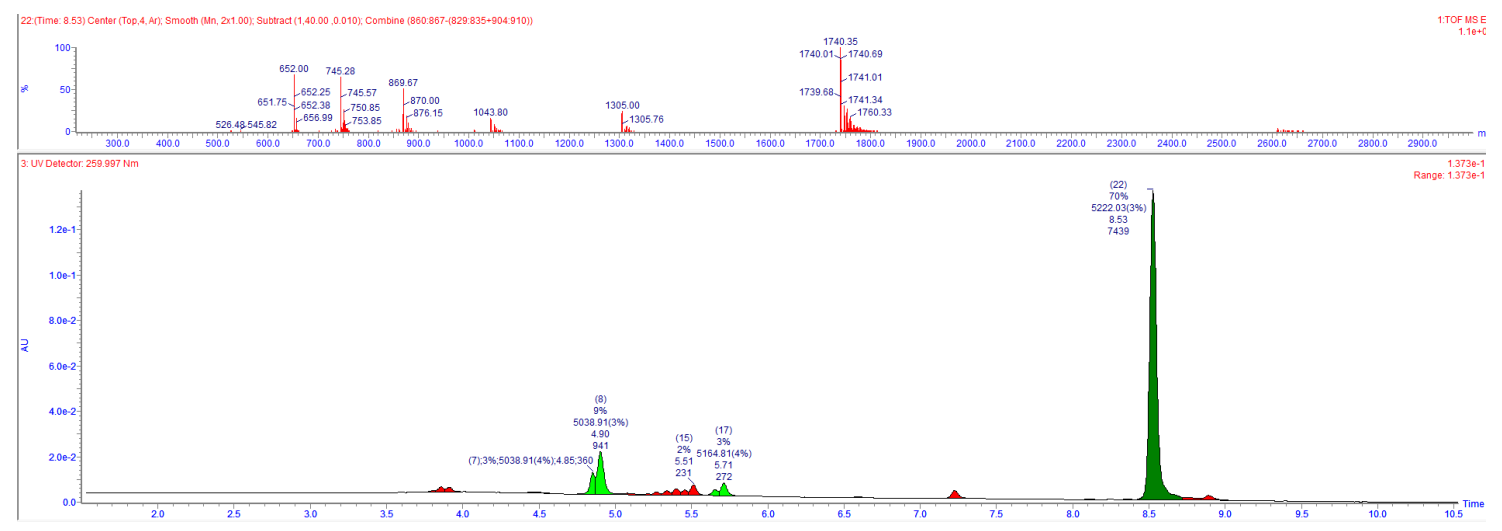




\section{LC Trace and Mass of 5}

Following General Procedure 5A with 1

Yield: $56 \%$

Exact mass: 5175.99

Triply charged mass [M-3]/3, caluculated: 1757.66; observed 1757.66
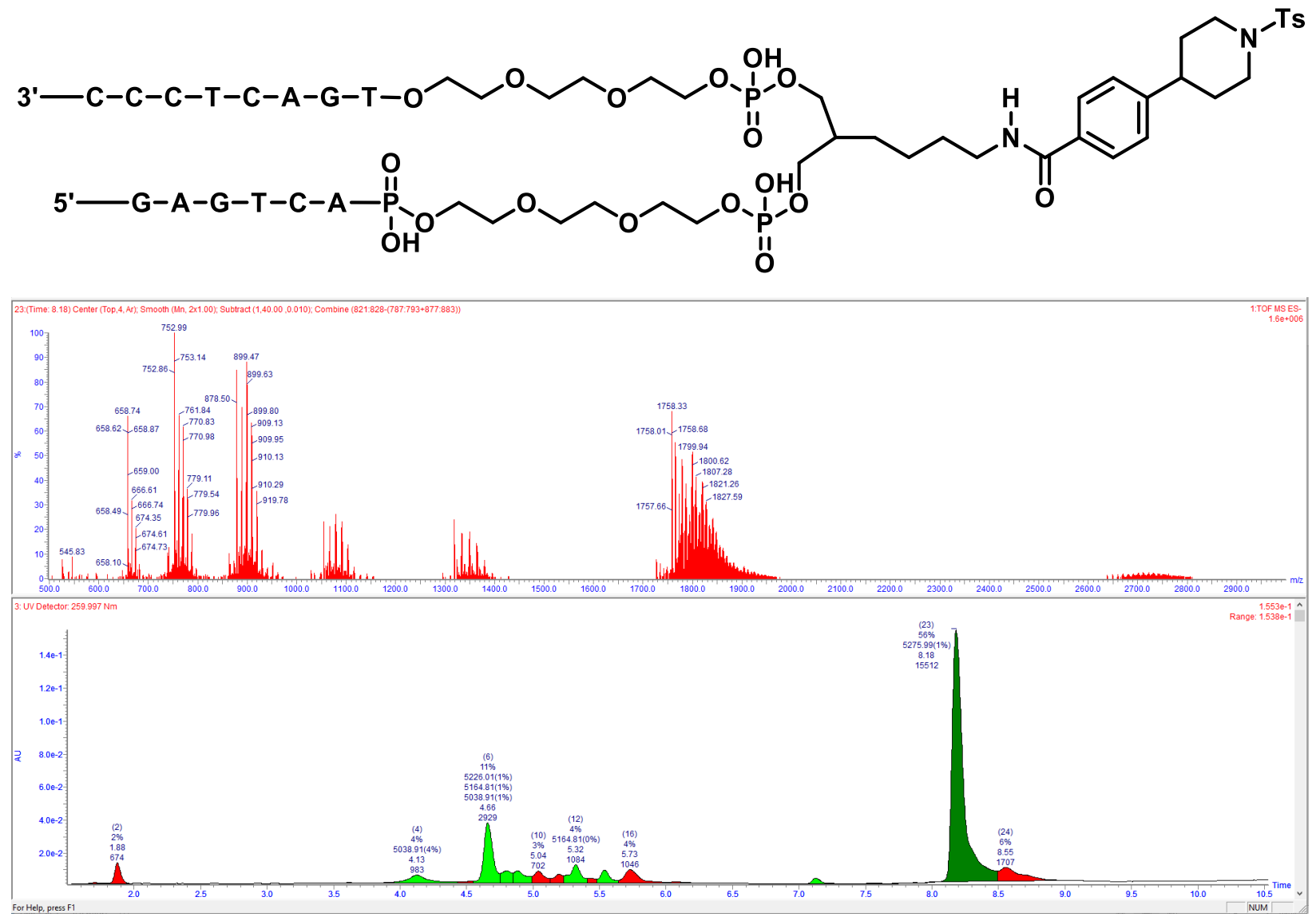


\section{LC Trace and Mass of 5 in tripliacate}

Following General Procedure 5B with 1

Yields: 64\%, 63\%, 51\%

Average Yield: 59.33\%

SD: 7.2

Exact masses: 5275.99

Triply charged masses [M-3]/3, caluculated: 1757.66; observed 1757.66, 1757.66, 1757.66
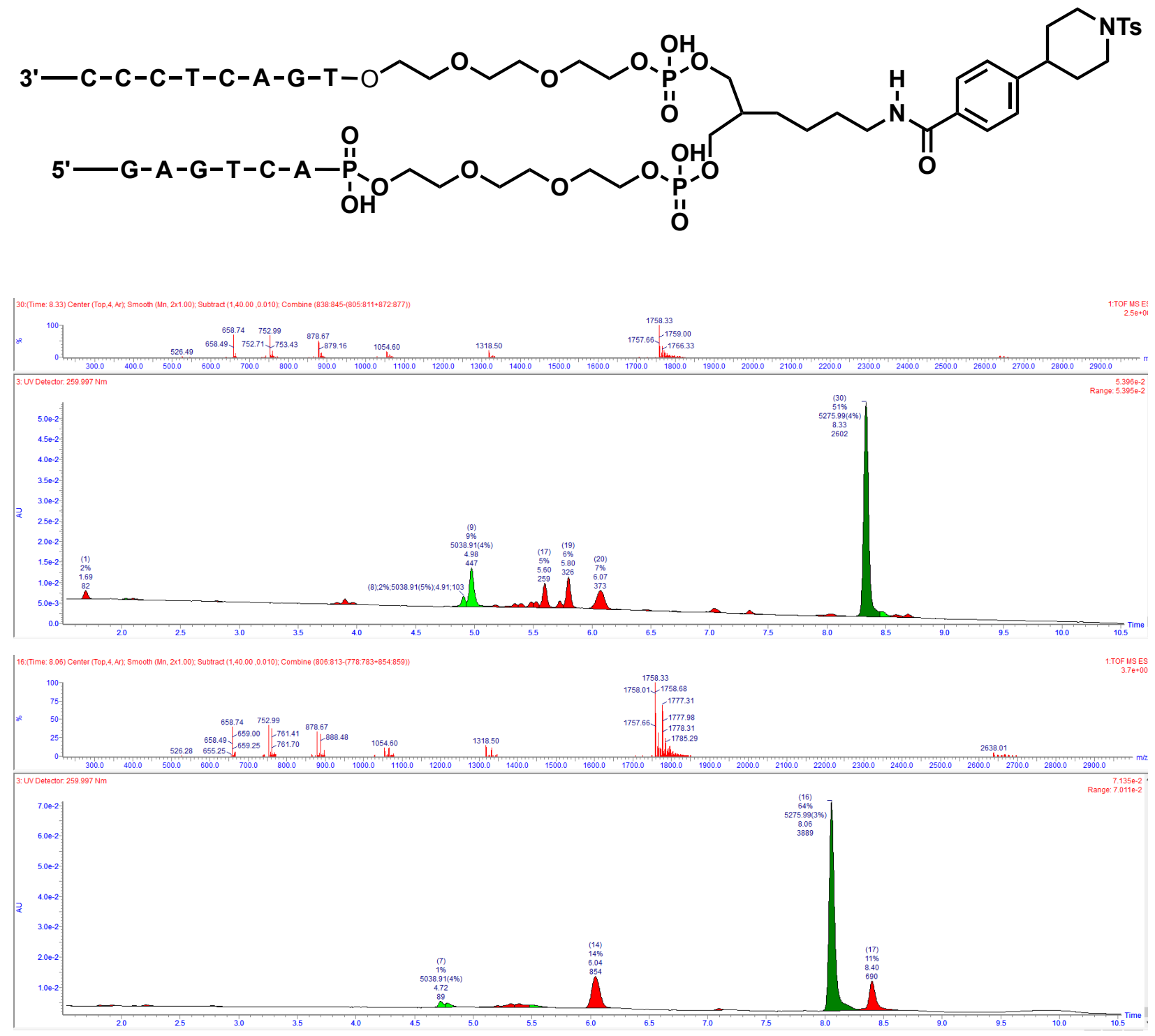


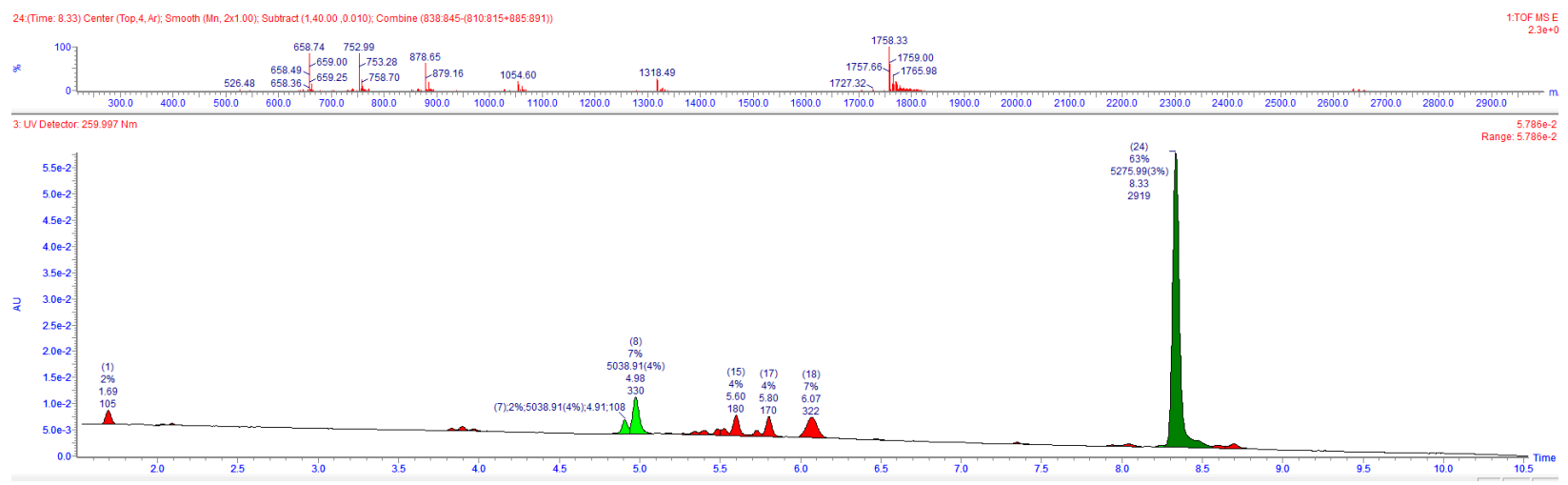




\section{LC Trace and Mass of 6}

Following General Procedure 5A with 1

Yield: $65 \%$

Exact mass: 5120.99

Triply charged mass [M-3]/3, caluculated: 1706.00; observed 1705.99
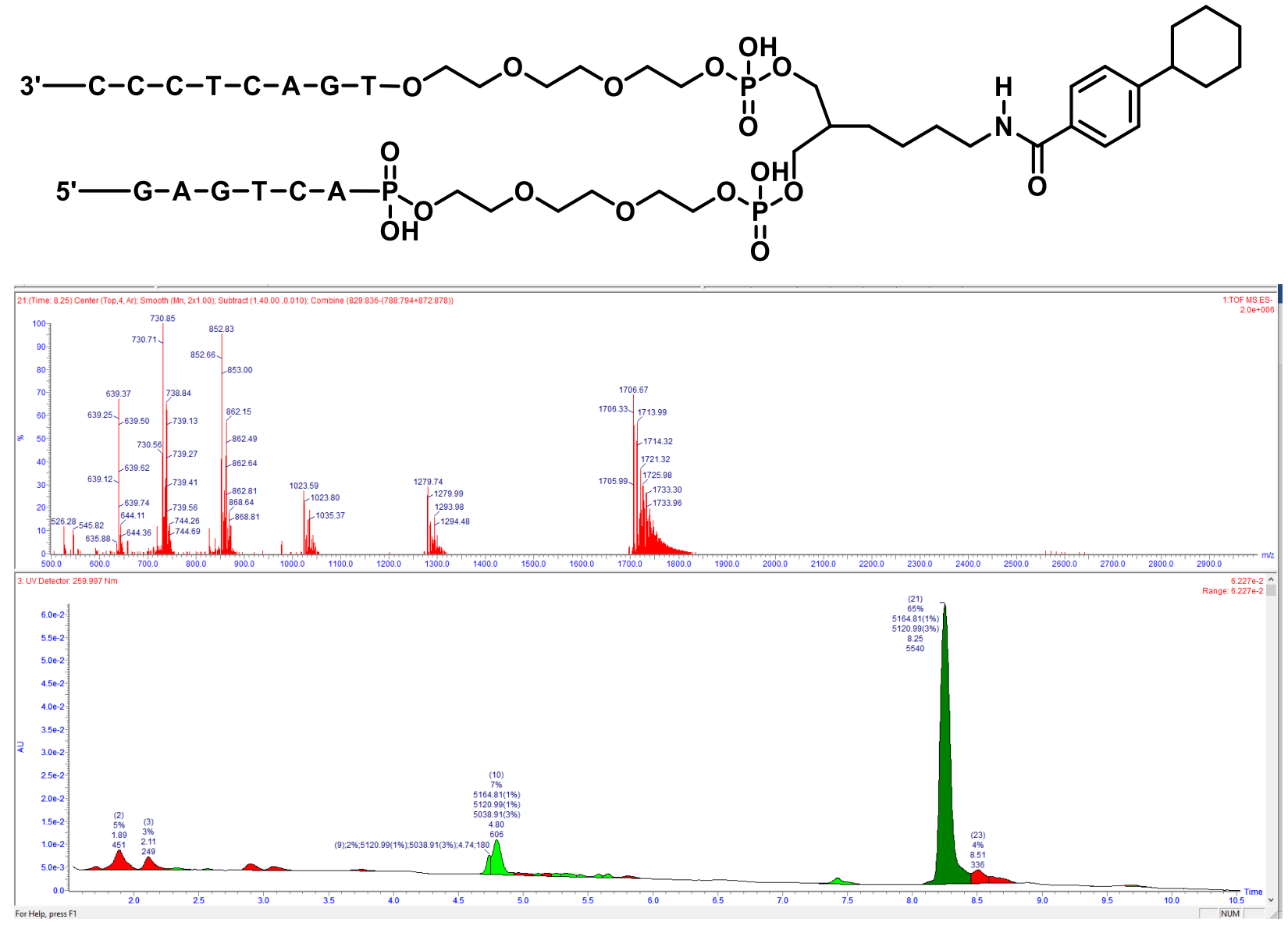


\section{LC Trace and Mass of 6 in tripliacate}

Following General Procedure 5B with 1

Yields: 70\%, 57\%, 60\%

Average Yield: $62.33 \%$

SDs: 6.8

Exact masses: 5120.99

Triply charged masses [M-3]/3, caluculated: 1705.99; observed 1705.99, 1705.99, 1705.99

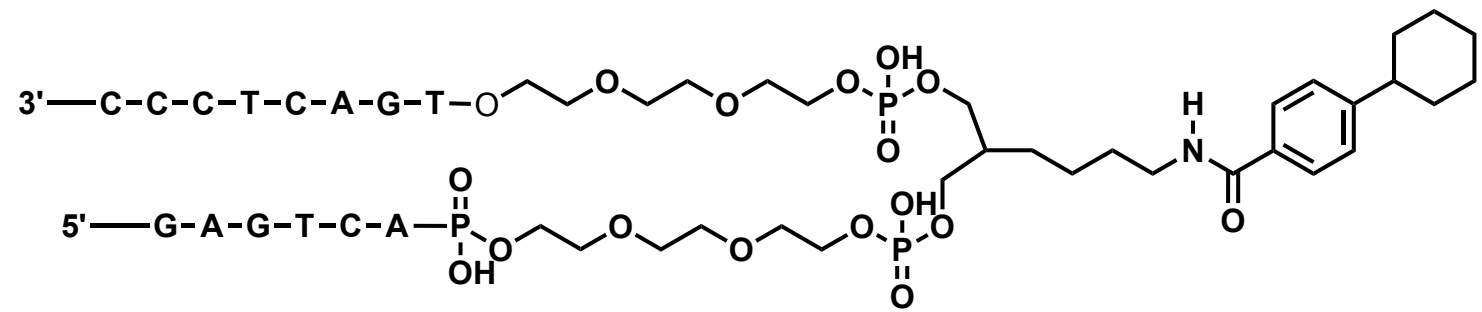
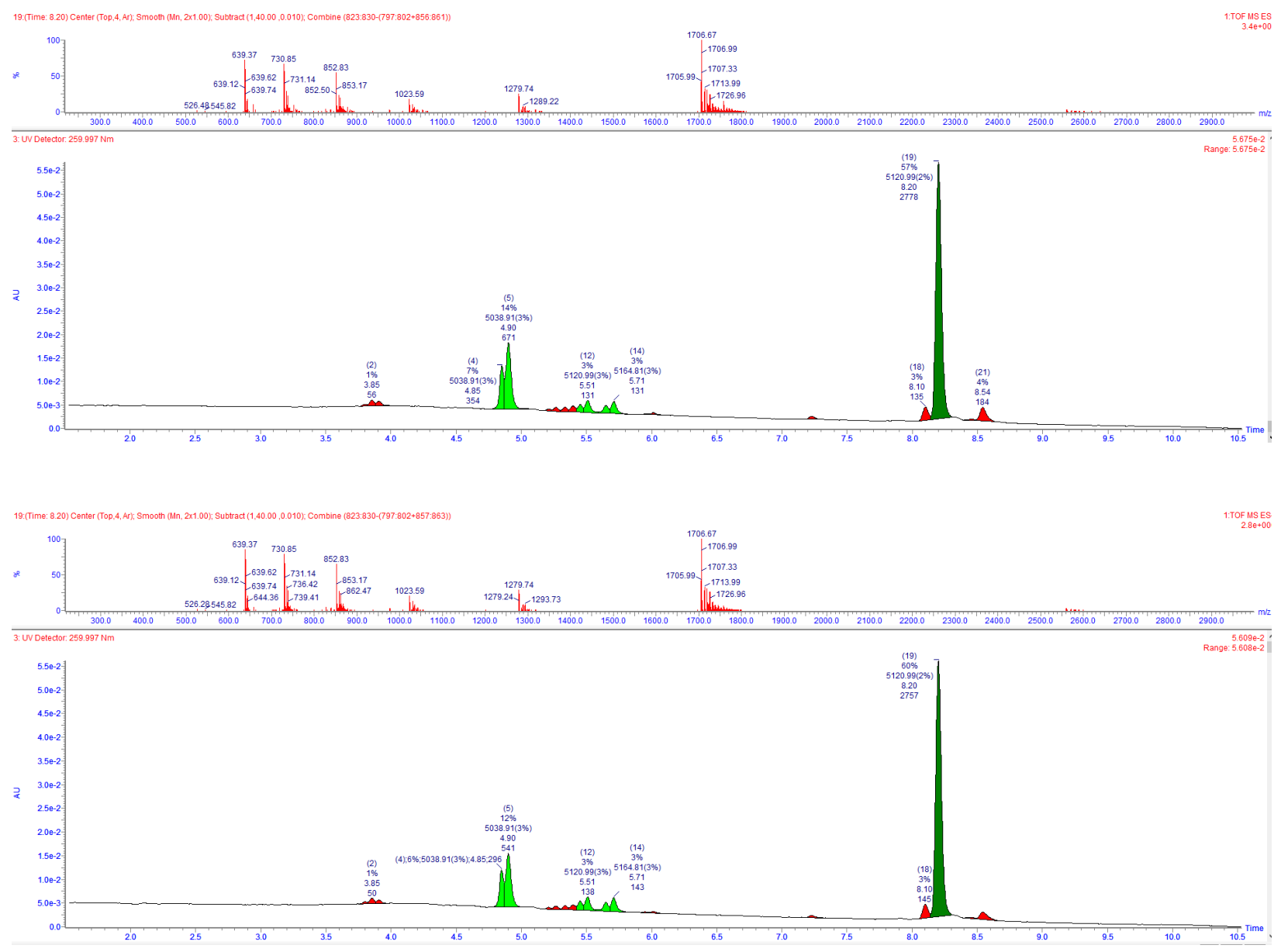


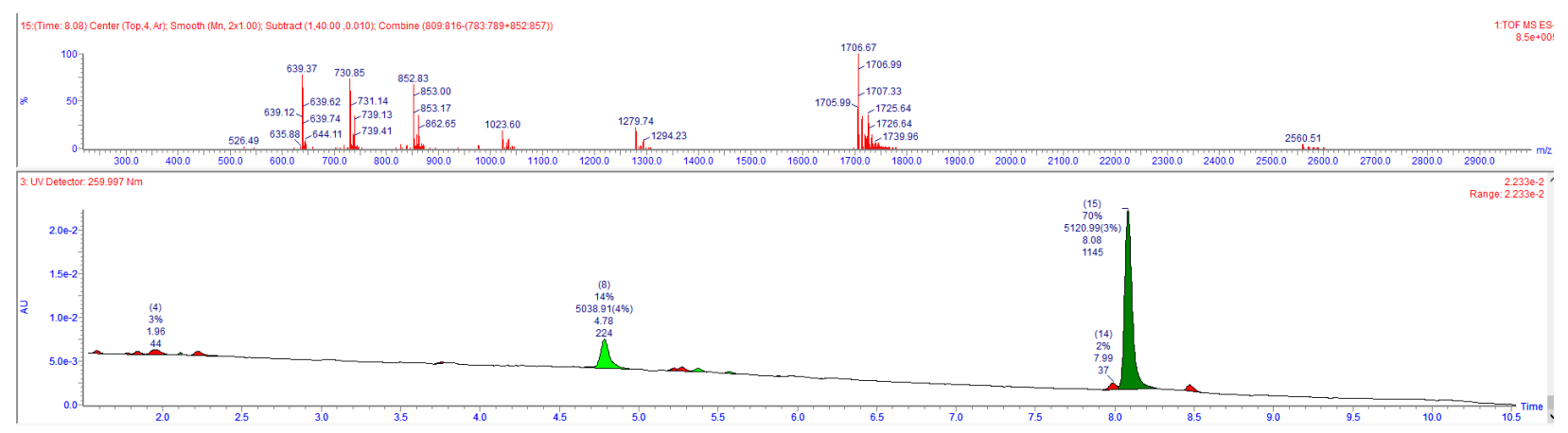




\section{LC Trace and Mass of 7 in tripliacate}

\section{Following General Procedure 5B with 1}

Yields: $62 \%, 70 \%, 71 \%$

Average Yield: $67.67 \%$

SDs: 4.9

Exact masses: 5156.97

Triply charged masses [M-3]/3, caluculated: 1719.99; observed 1719.99, 1719.99. 1718.99
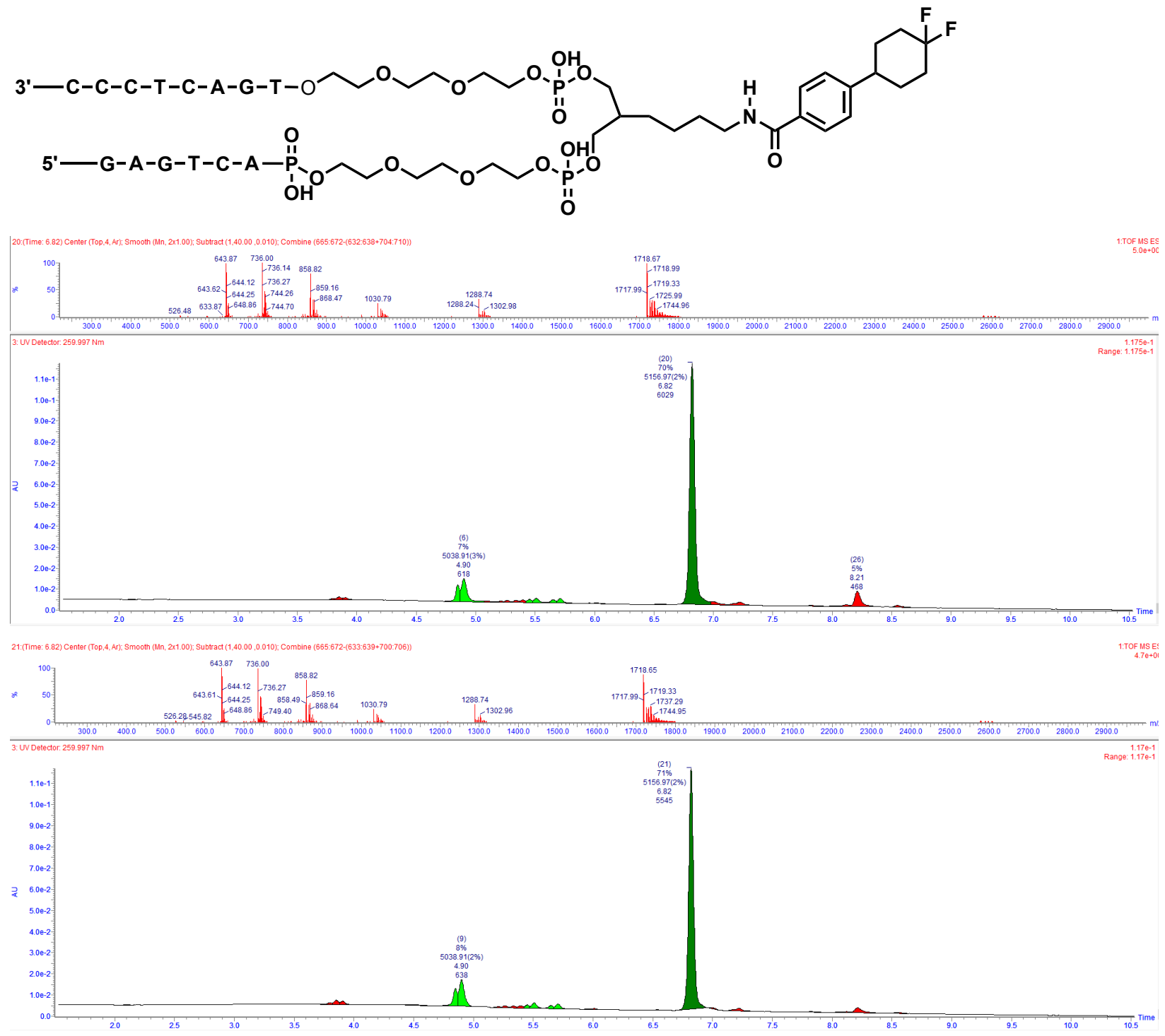


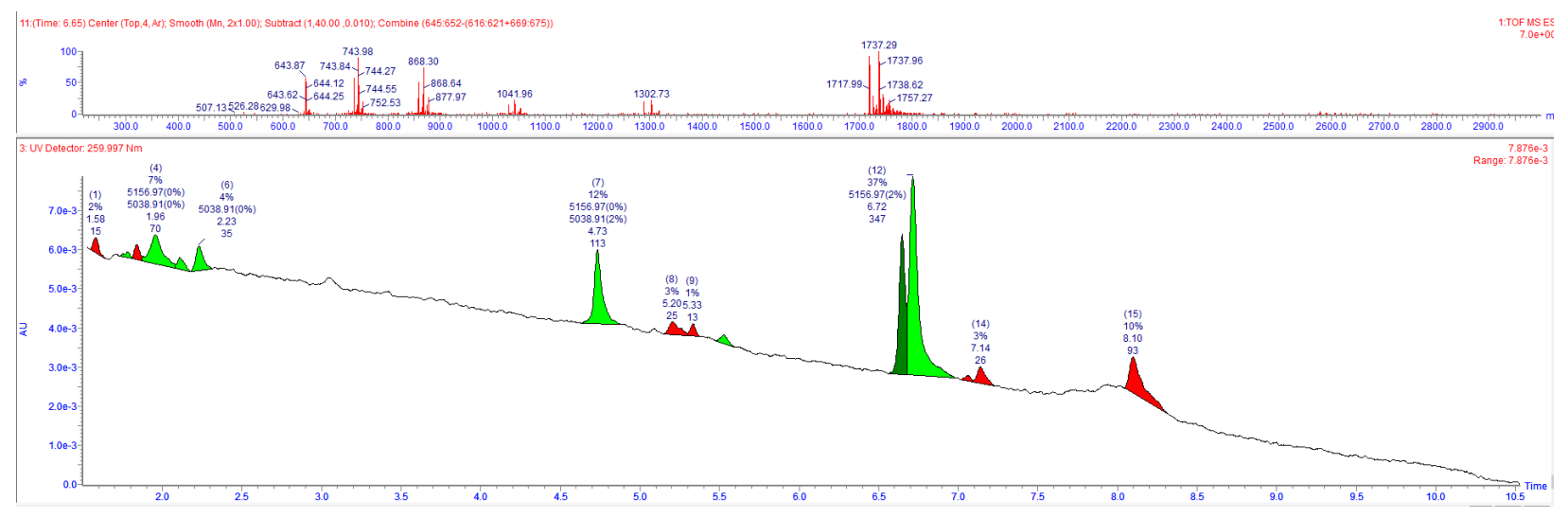




\section{LC Trace and Mass of 8 in tripliacate}

Following General Procedure 5B with 1

Yields: 50\%, 67\%, 39\%

Average Yield: 522\%

SDs: 14

Exact masses: 5122.97

Triply charged masses [M-3]/3, caluculated: 1706.65; observed $1706.65,1706.65$
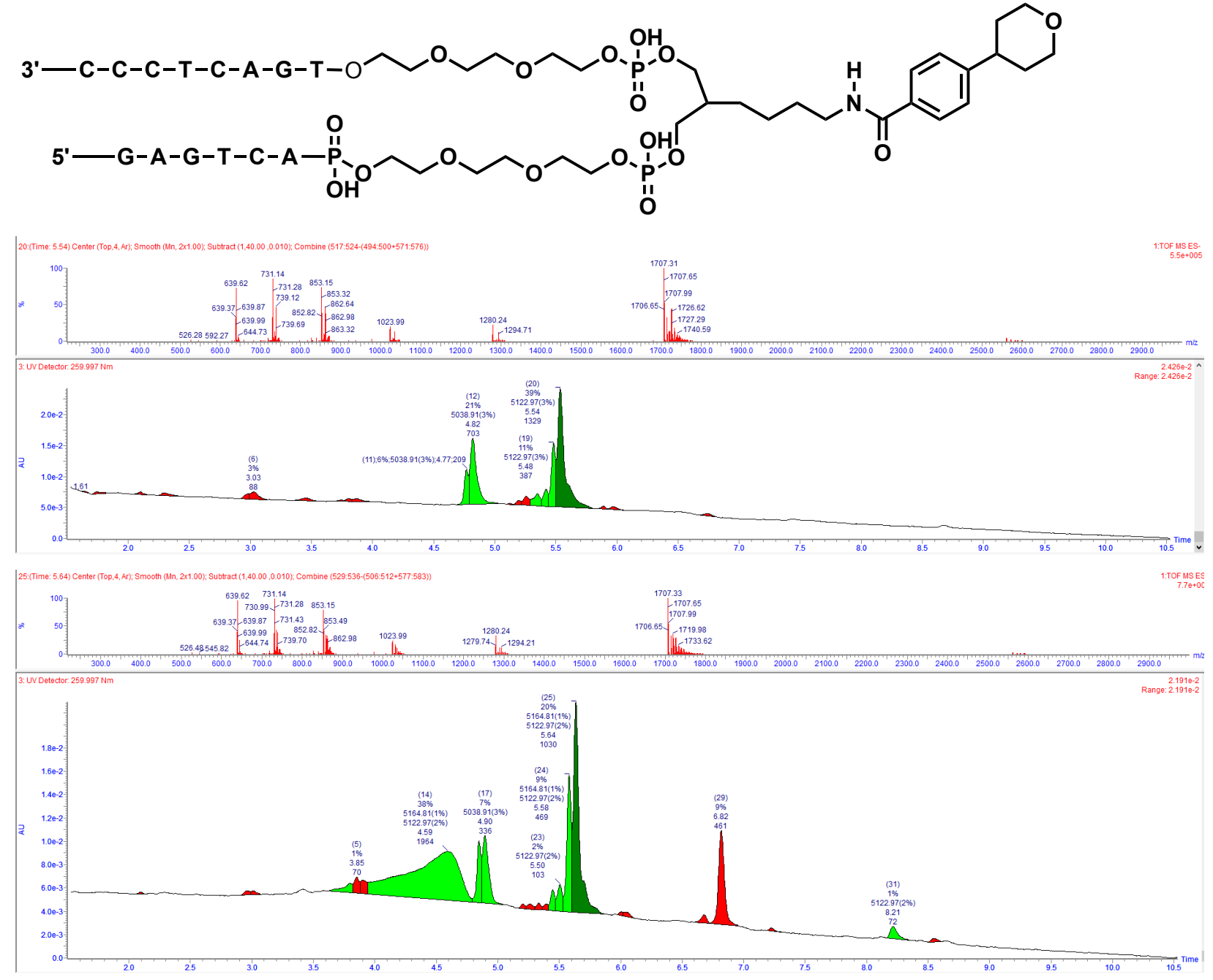


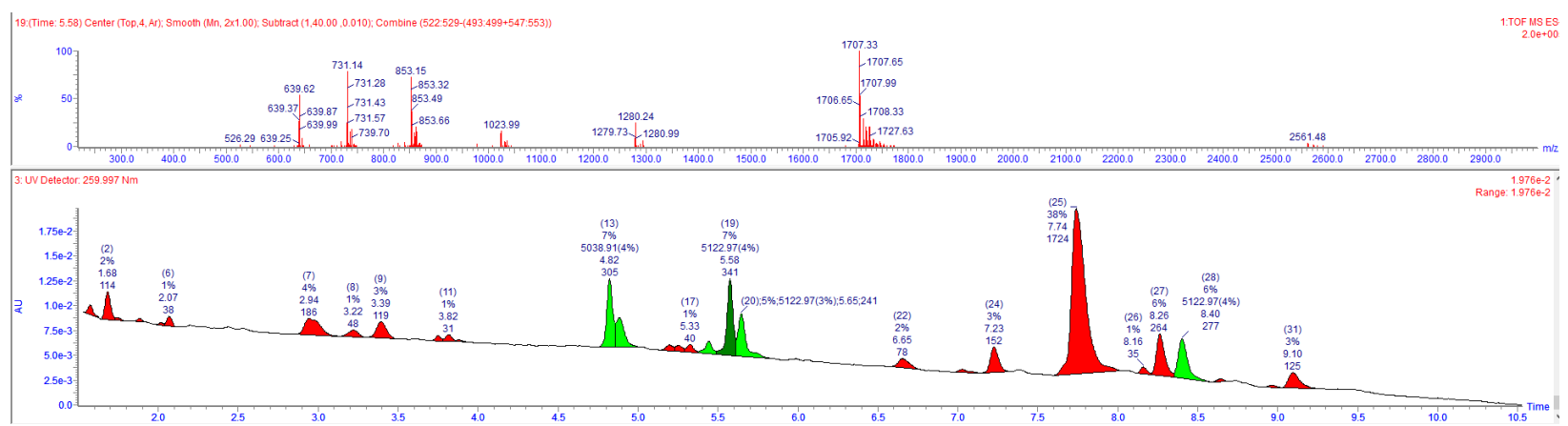




\section{LC Trace and Mass of 9}

Following General Procedure 5A with $\mathbf{1}$

Yield: $78 \%$

Exact mass: 5194.00

Triply charged mass [M-3]/3, caluculated: 1730.33; observed 1730.33
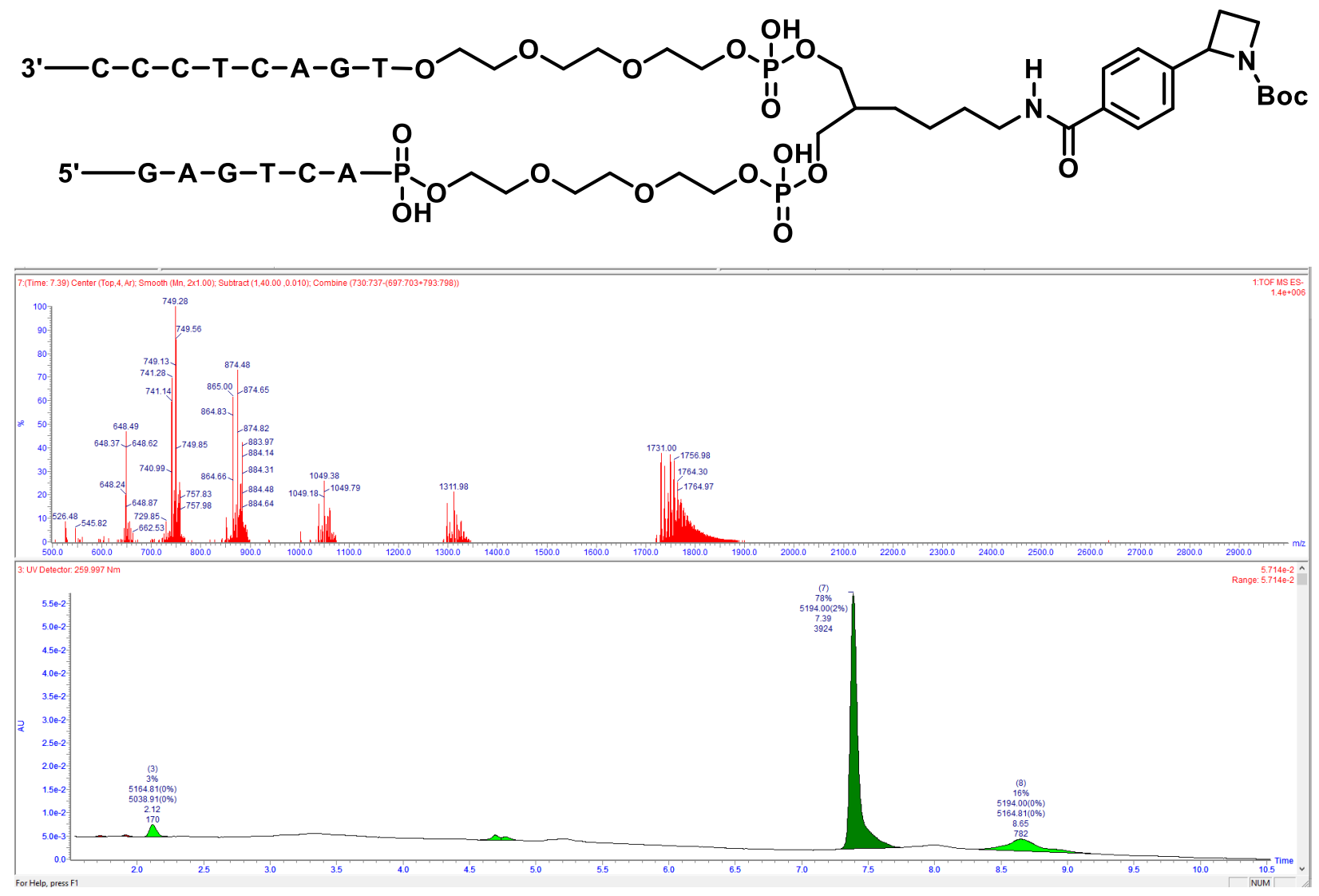


\section{LC Trace and Mass of 9 in tripliacate}

Following General Procedure 5B with 1

Yields: $88 \%, 75 \%, 87 \%$

Average Yield: 83.33\%

SDs: 7.2

Exact masses: 5194.00

Triply charged masses [M-3]/3, caluculated: 1730.33; observed $1730.33,1730.33,1730.33$
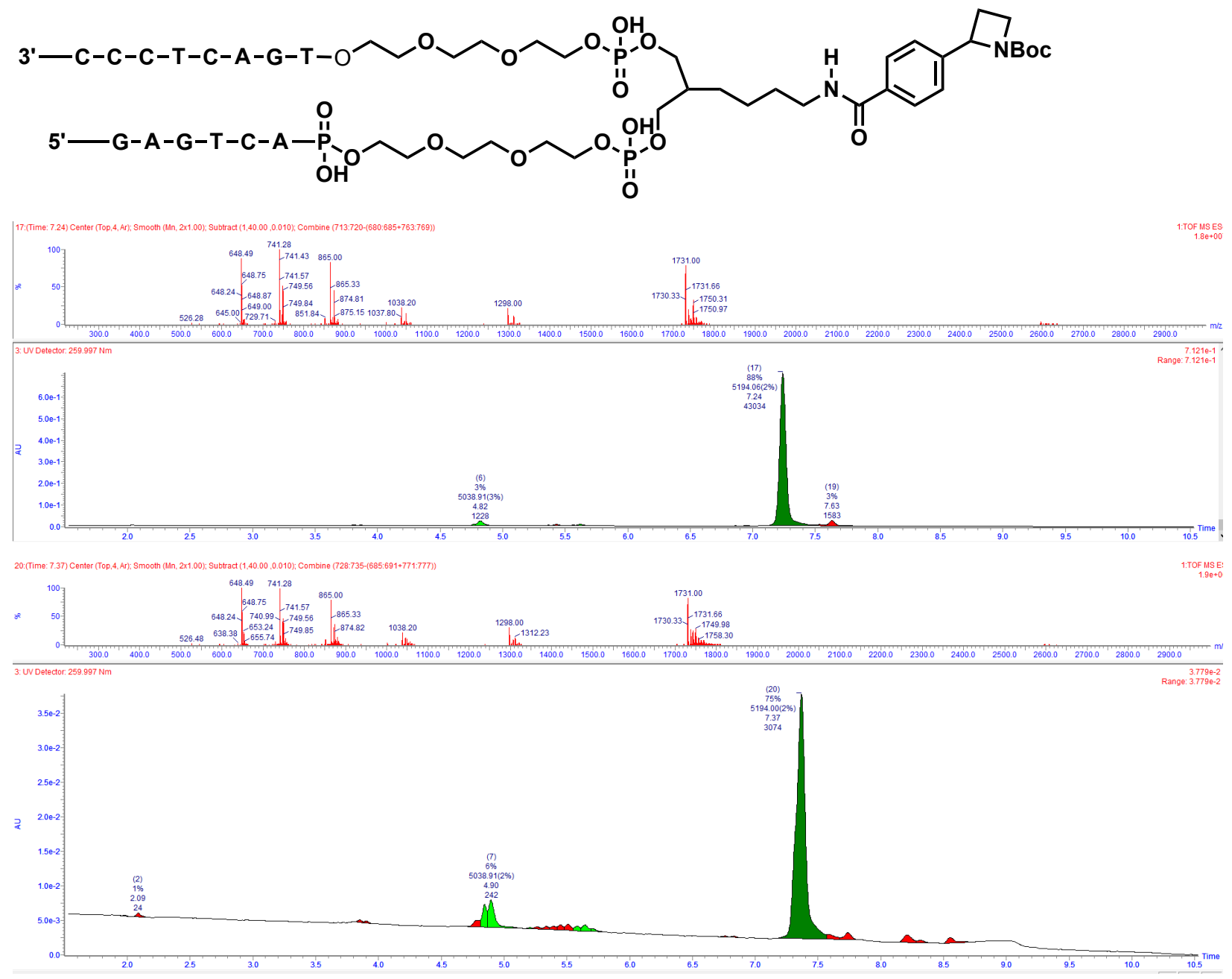


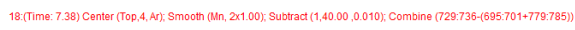

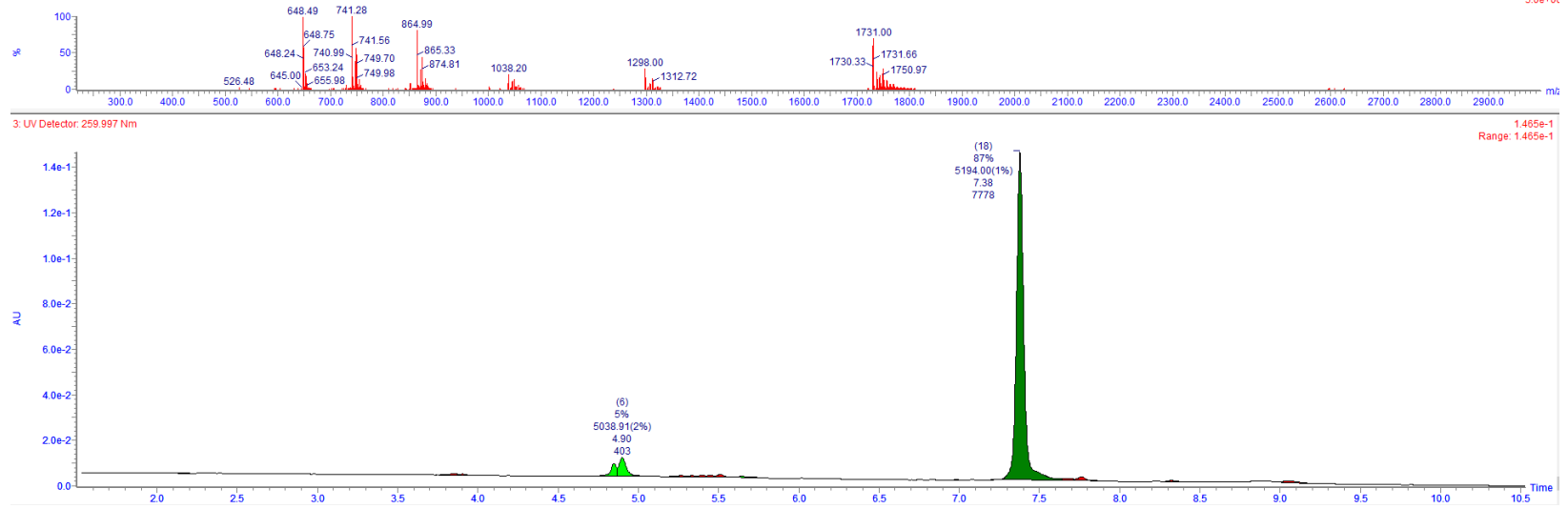




\section{LC Trace and Mass of 10}

Following General Procedure 5A with 1

Yield: $87 \%$

Exact mass: 5208.02

Triply charged mass [M-3]/3, caluculated: 1735.01; observed 1735.00
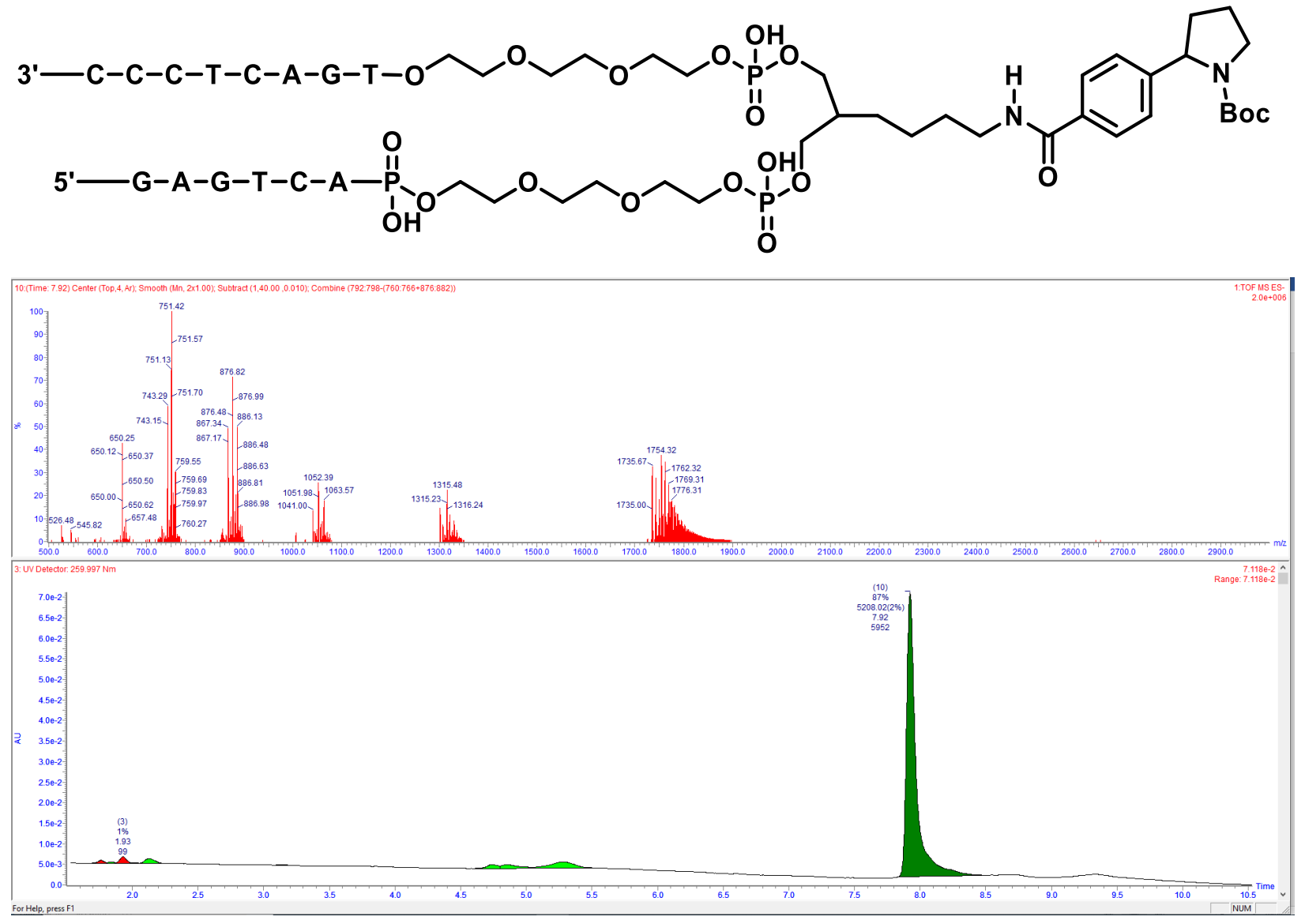


\section{LC Trace and Mass of 10 in tripliacate}

Following General Procedure 5B with 1

Yields: 94\%, 70\%, 76\%

Average Yield: 80\%

SD: 12.5

Exact mass: 5208.02

Triply charged masses [M-3]/3, caluculated: 1735.00; observed 1735.00
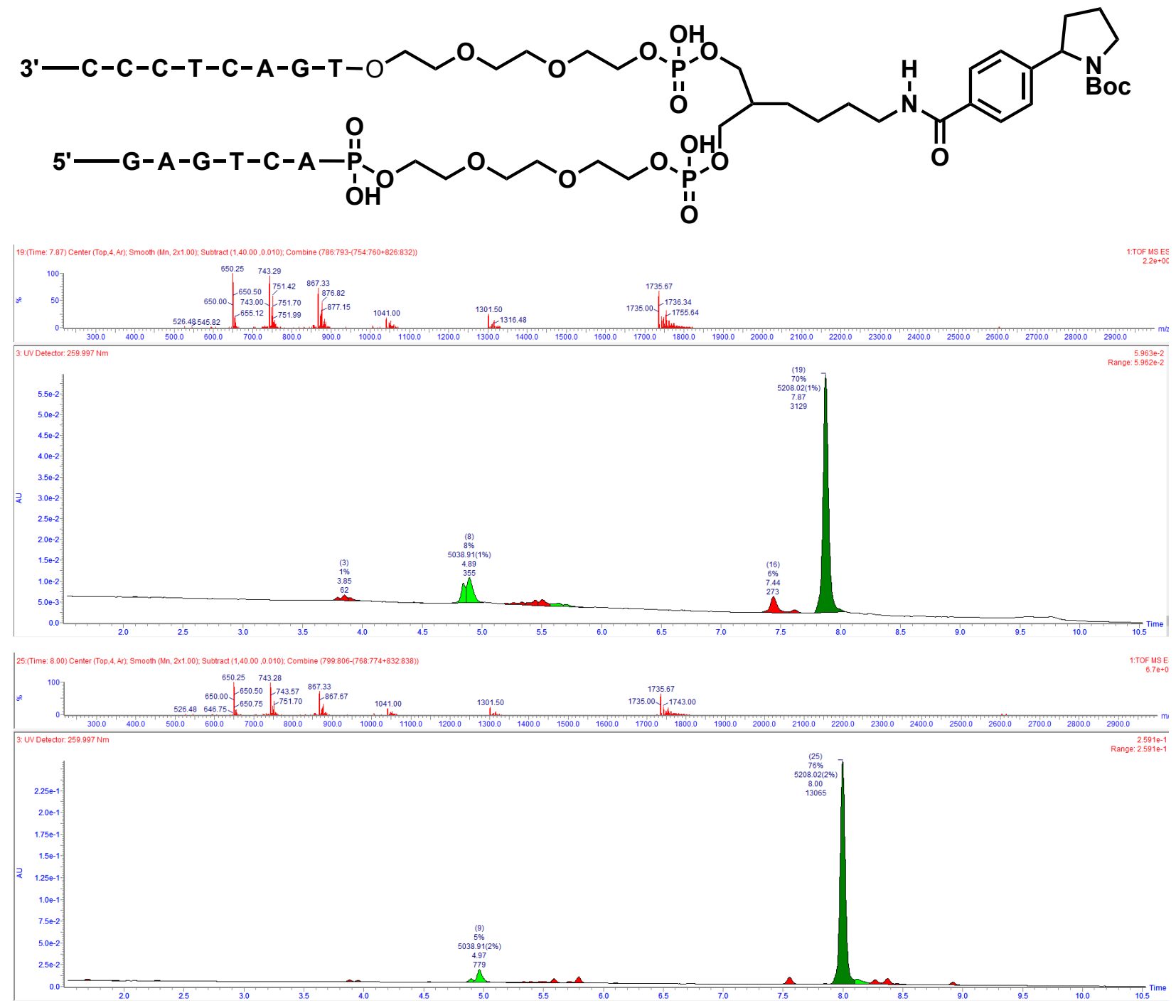


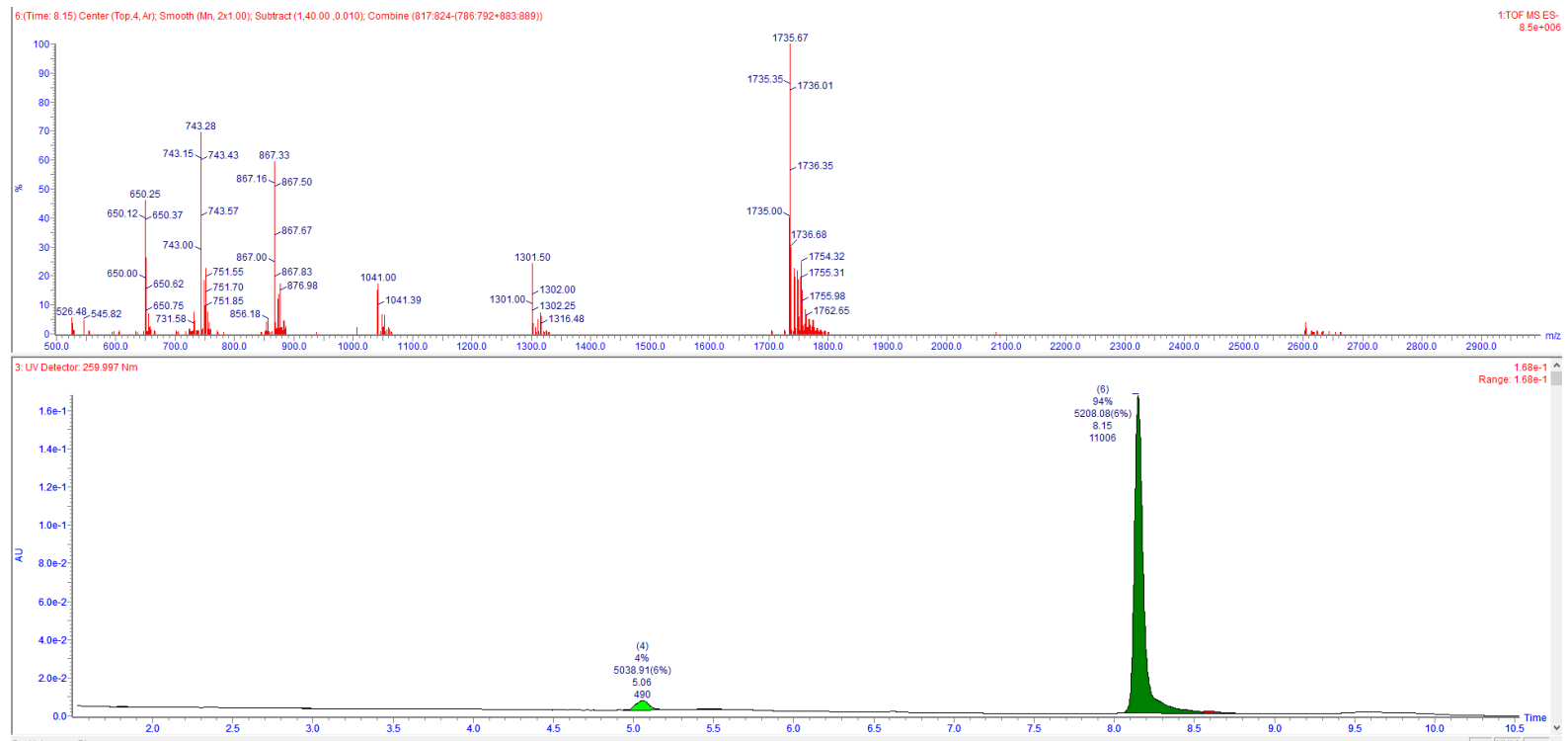




\section{LC Trace and Mass of 11}

Following General Procedure 5A with $\mathbf{1}$

Yield: $80 \%$

Exact mass: 5225.98

Triply charged mass [M-3]/3, caluculated: 1740.99; observed 1741.00
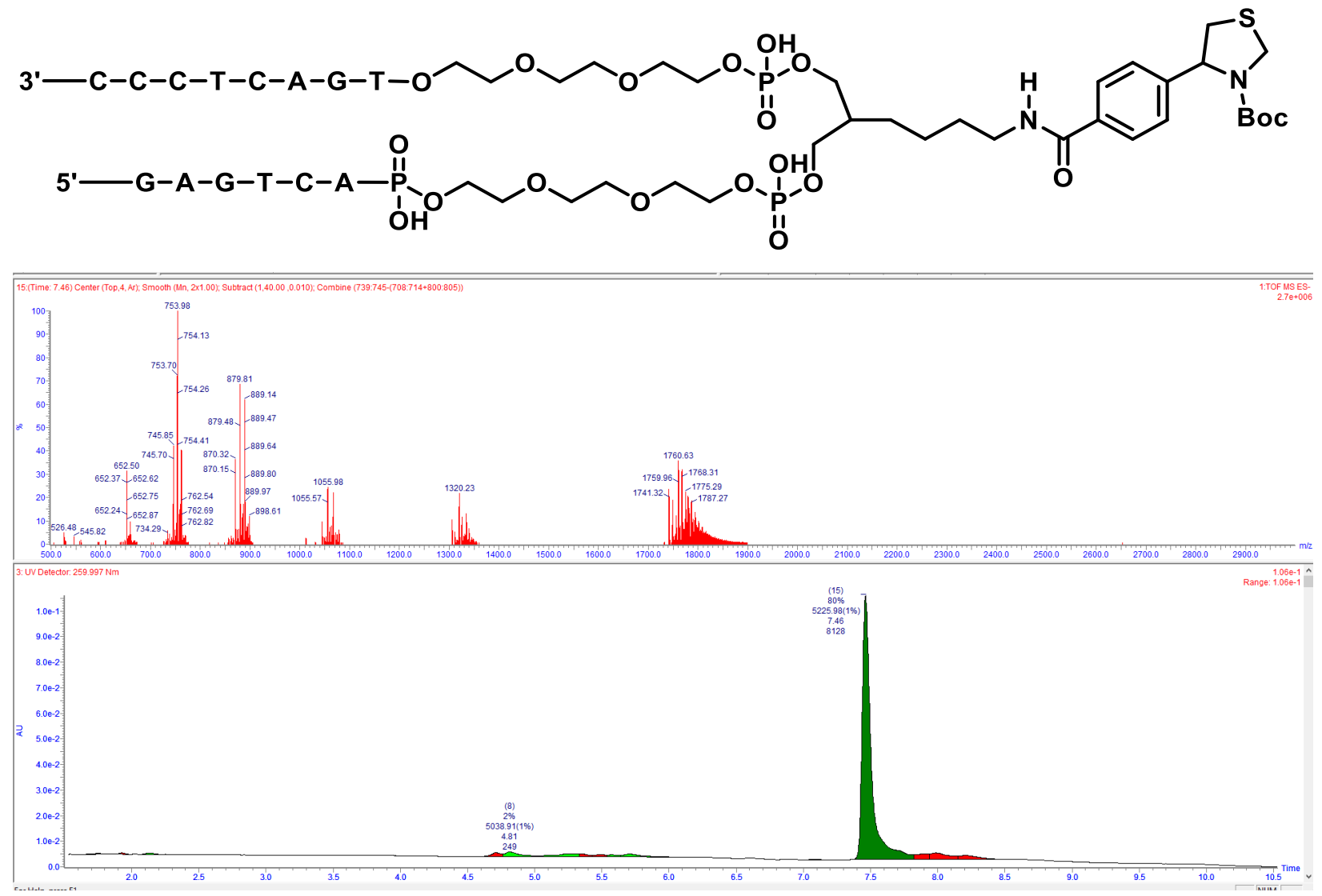


\section{LC Trace and Mass of 11 in tripliacate}

\section{Following General Procedure 5B with 1}

Yields: 78\%, 73\%, 74\%

Average Yield: $75 \%$

SDs: 2.64

Exact mass: 5225.98

Triply charged masses [M-3]/3, caluculated: 1740.99; observed $1741.00,1740.98,1740.99$
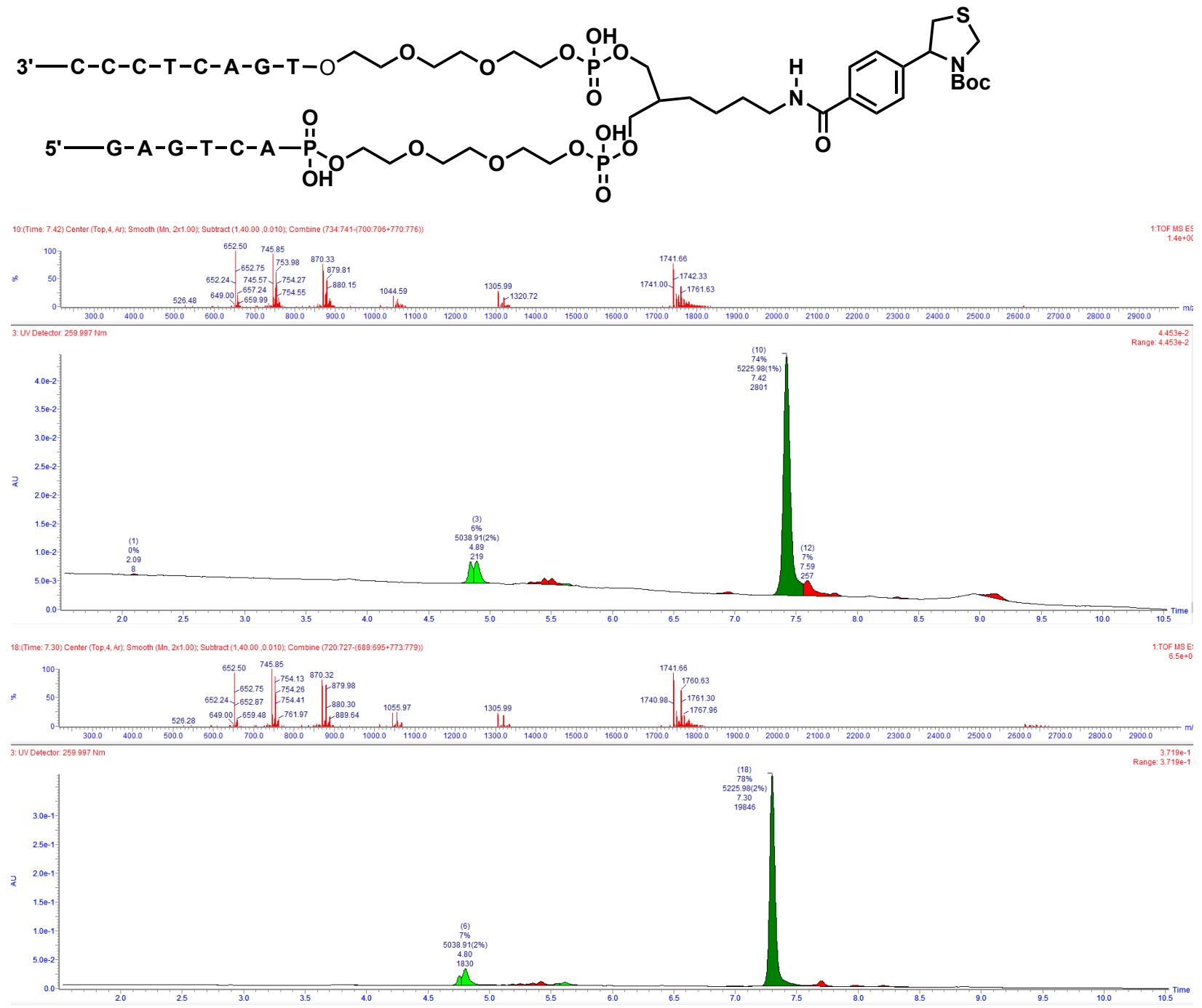


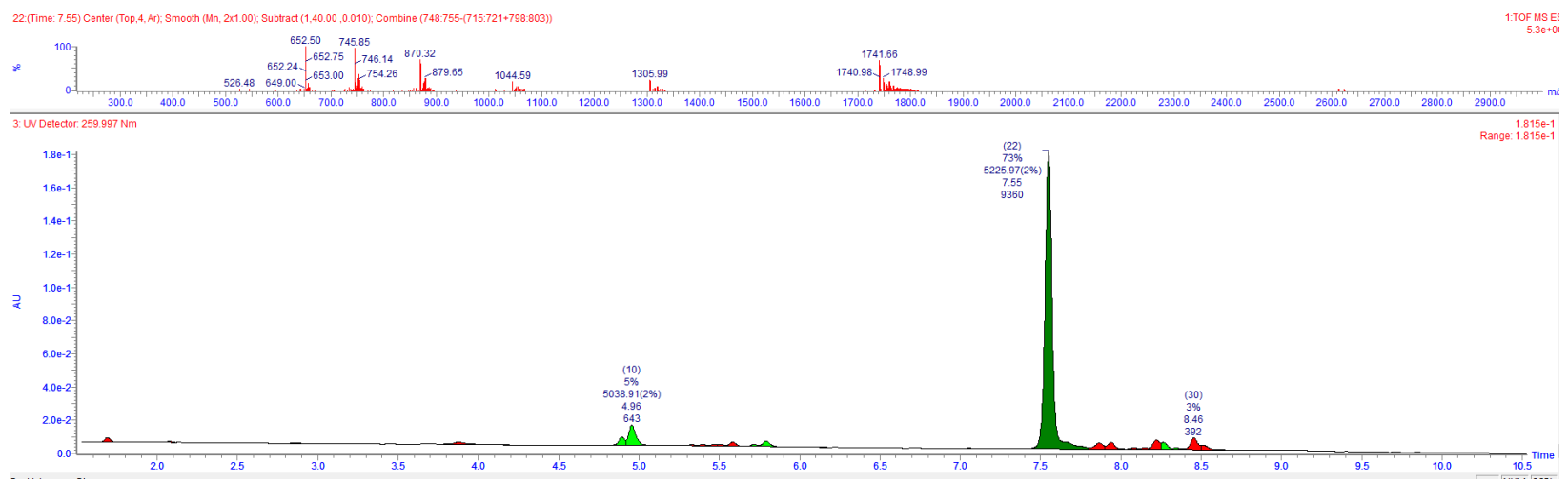




\section{LC Trace and Mass of 12 in tripliacate}

Following General Procedure 5B with 1

Yields: 55\%, 54\%, 73\%

Average Yield: 60.66\%

SD: 10.7

Exact mass: 5108.95

Triply charged masses [M-3]/3, caluculated: 1700.98; observed $1701.999,1701.00$
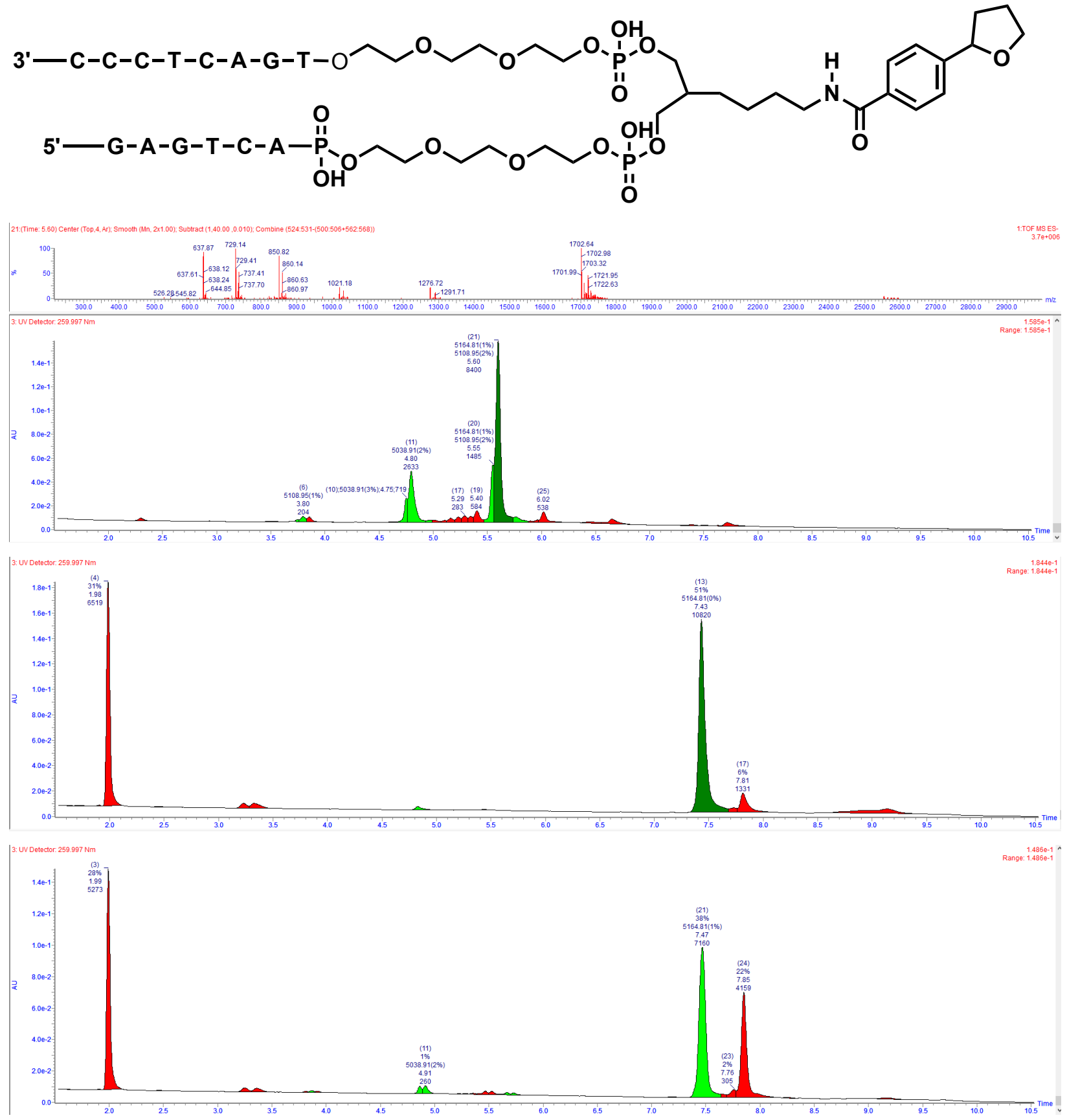


\section{LC Trace and Mass of 13 in tripliacate}

Following General Procedure 5A with 1

Yields: 77\%, 64\%, 55\%

Average Yield: $65.33 \%$

SD: 11

Exact mass: 5120.99

Triply charged masses [M-3]/3, caluculated: 1705.99; observed $1705.99,1705.99,1706.99$
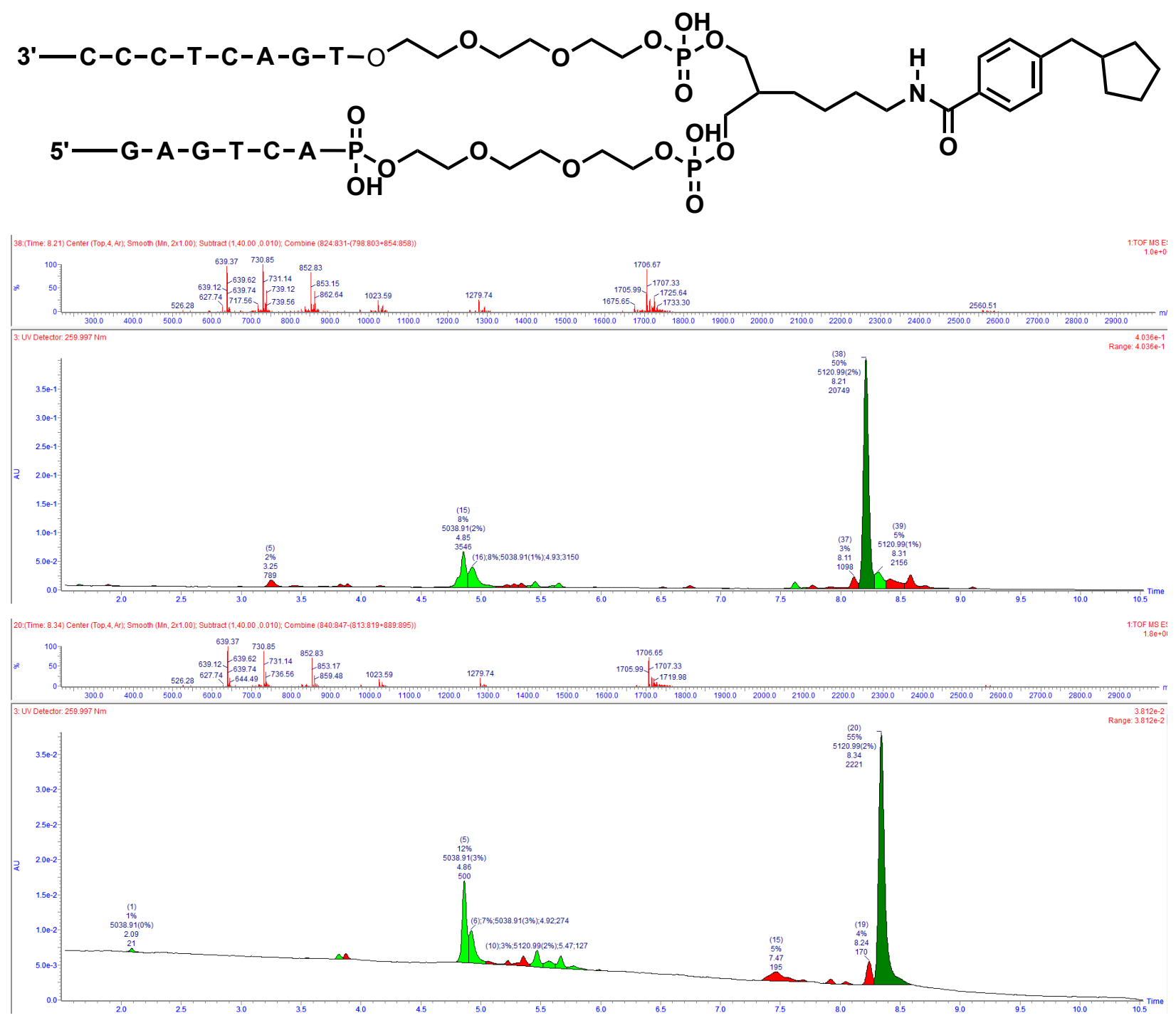


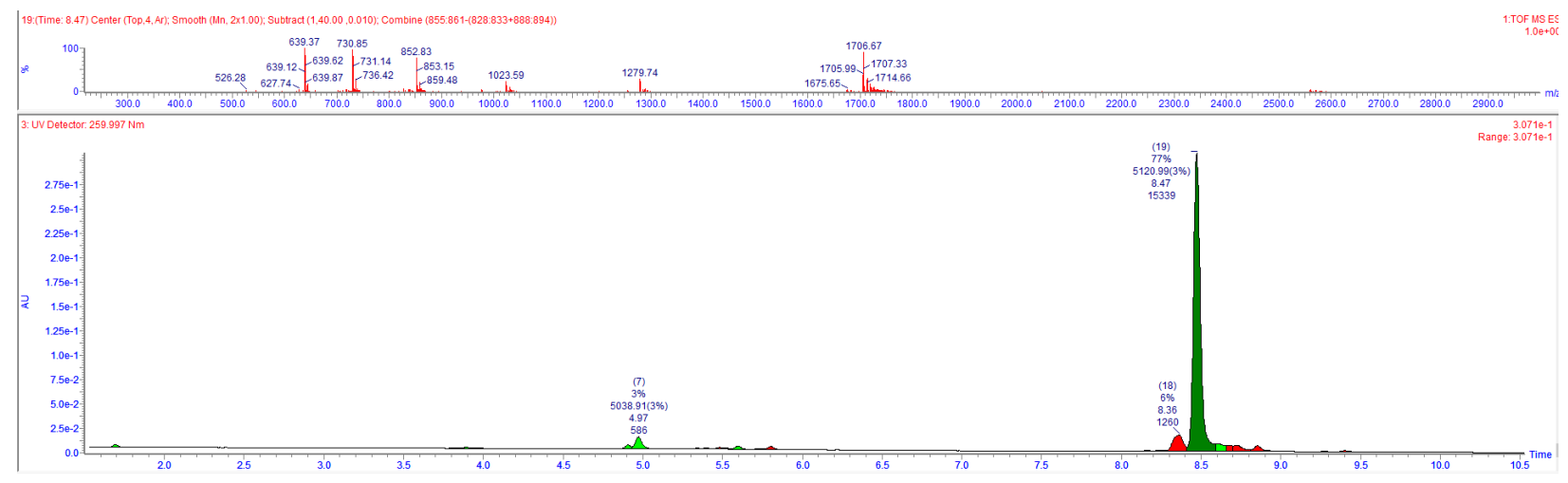




\section{LC Trace and Mass of 14}

Following General Procedure 5A with 1

Yield: $65 \%$

Exact mass: 5240.83

Triply charged mass [M-3]/3, caluculated: 1745.94; observed 1745.94
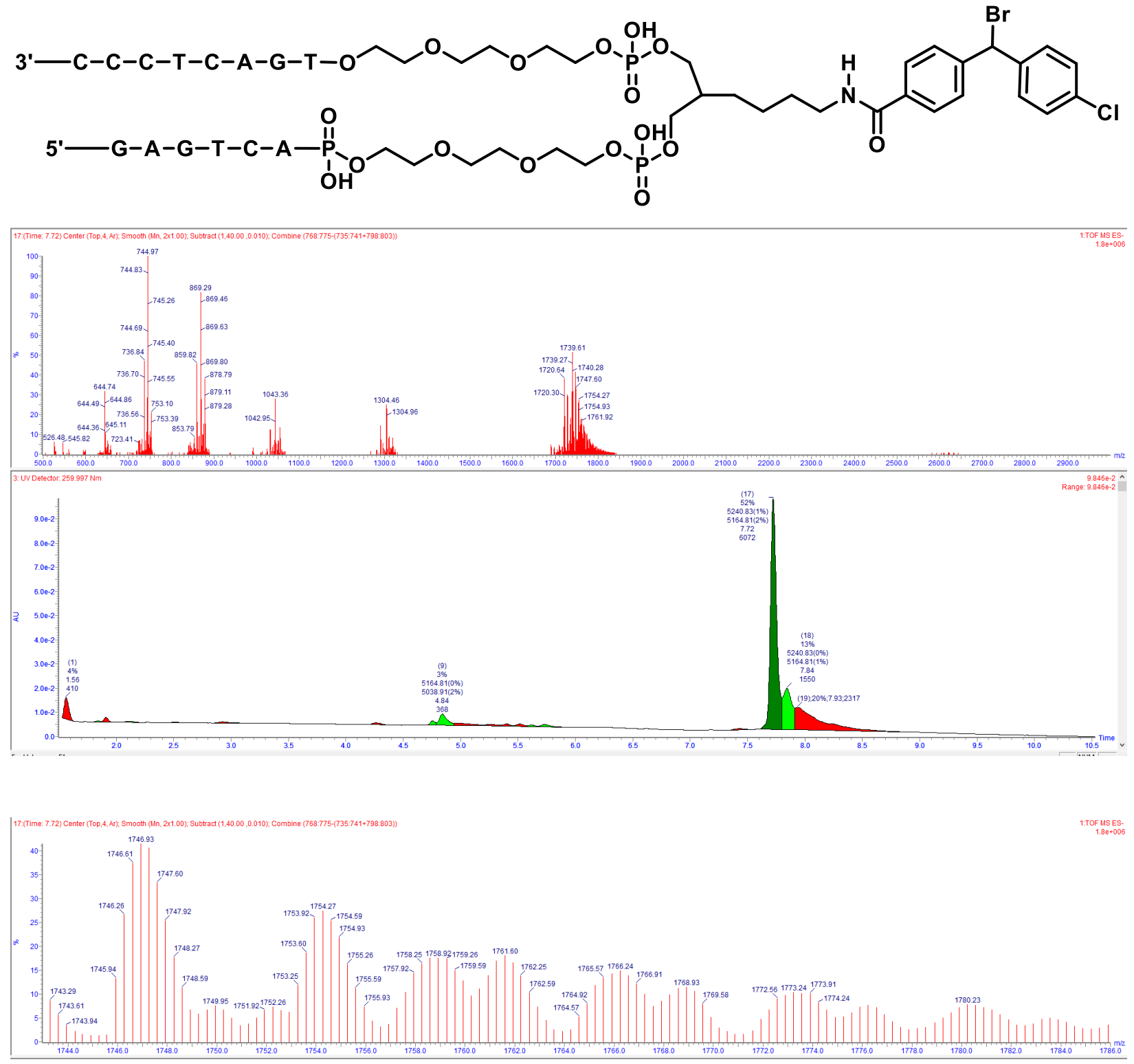


\section{LC Trace and Mass of 14 in tripliacate}

\section{Following General Procedure 5B with 1}

Yields: $71 \%, 64 \%, 68 \%$

Average Yield: $67.66 \%$

SD: 3.51

Exact mass: 5220.88

Triply charged masses [M-3]/3, caluculated: 1739.29; observed $1739.61,1739.60,1738.6$
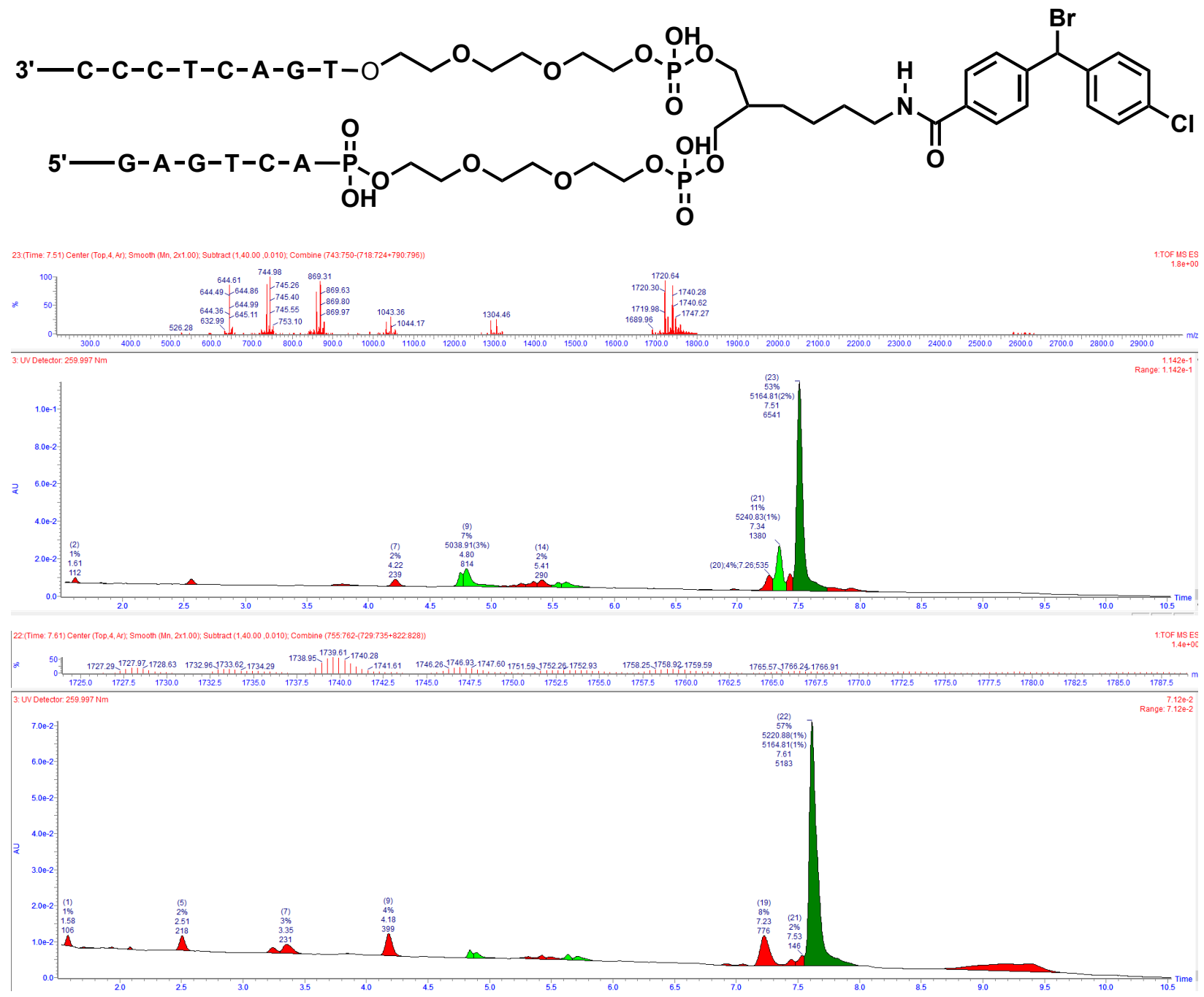


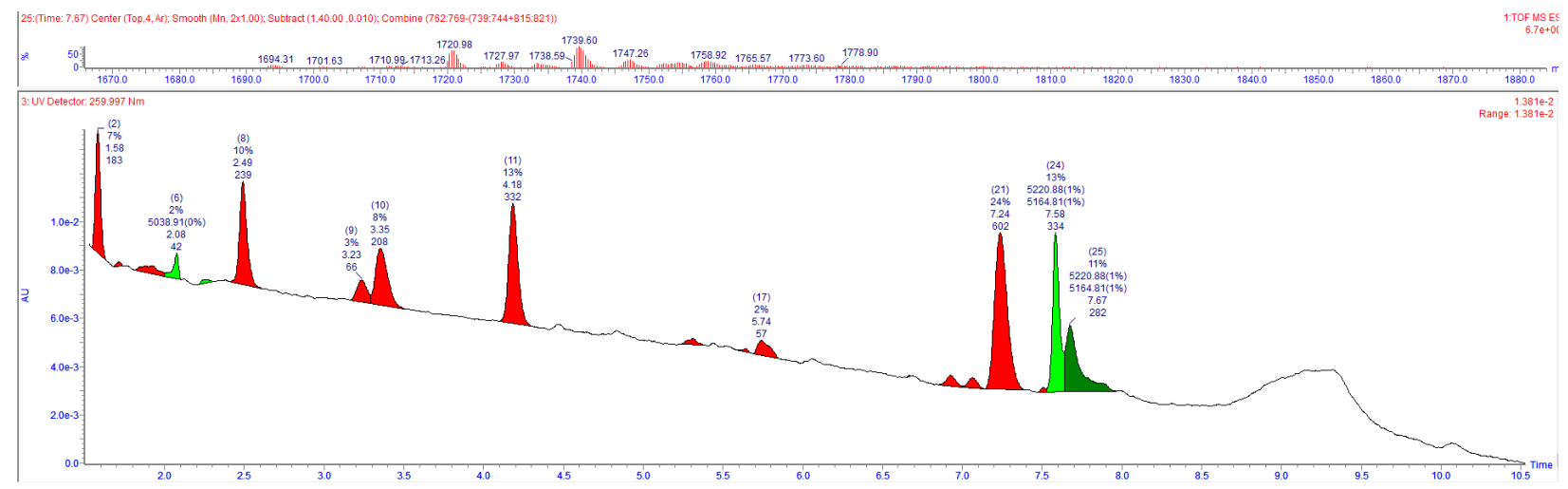




\section{LC Trace and Mass of 15}

Following General Procedure 5A with 1

Yield: $60 \%$

Exact mass: 5158.97

Triply charged mass [M-3]/3, caluculated: 1718.66; observed 1718.65
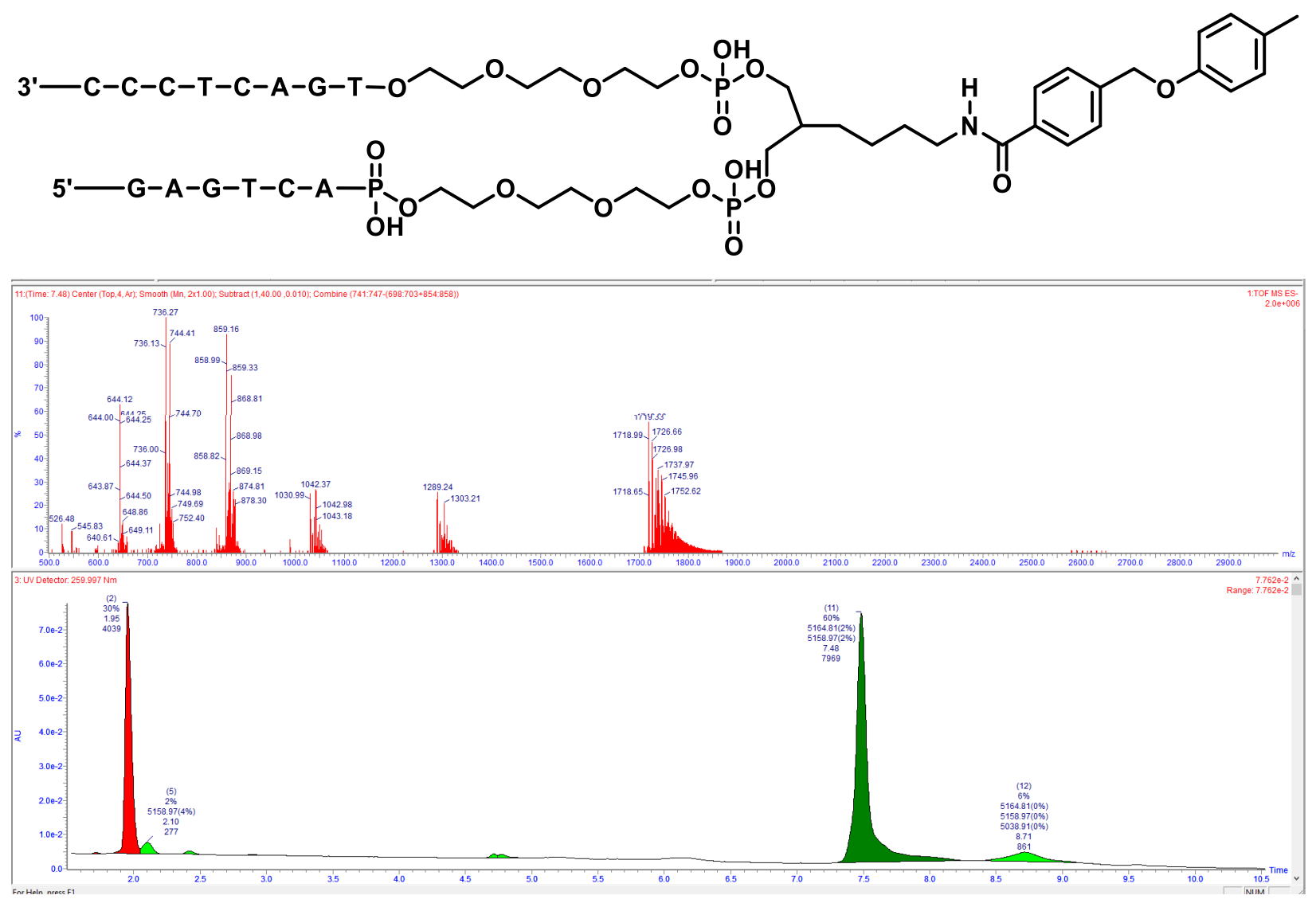


\section{LC Trace and Mass of 16 in tripliacate}

\section{Following General Procedure 5B with 1}

Yields: 64\%, 45\%, 41\%

Average Yield: 50\%

SD: 12.3

Exact mass: 5135.00

Triply charged masses [M-3]/3, caluculated: 1710.66; observed $1710.66,1710.66,1710.66$
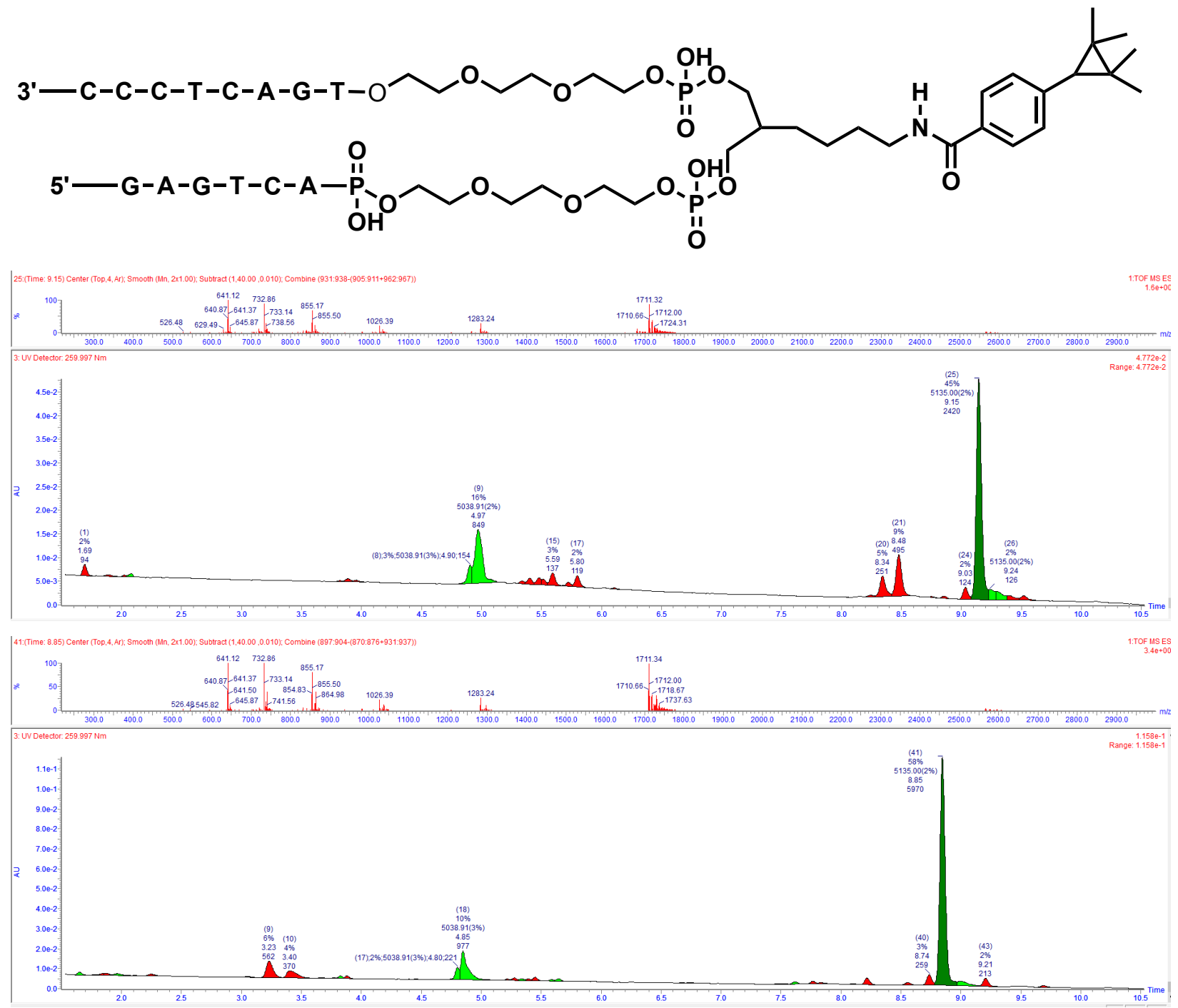


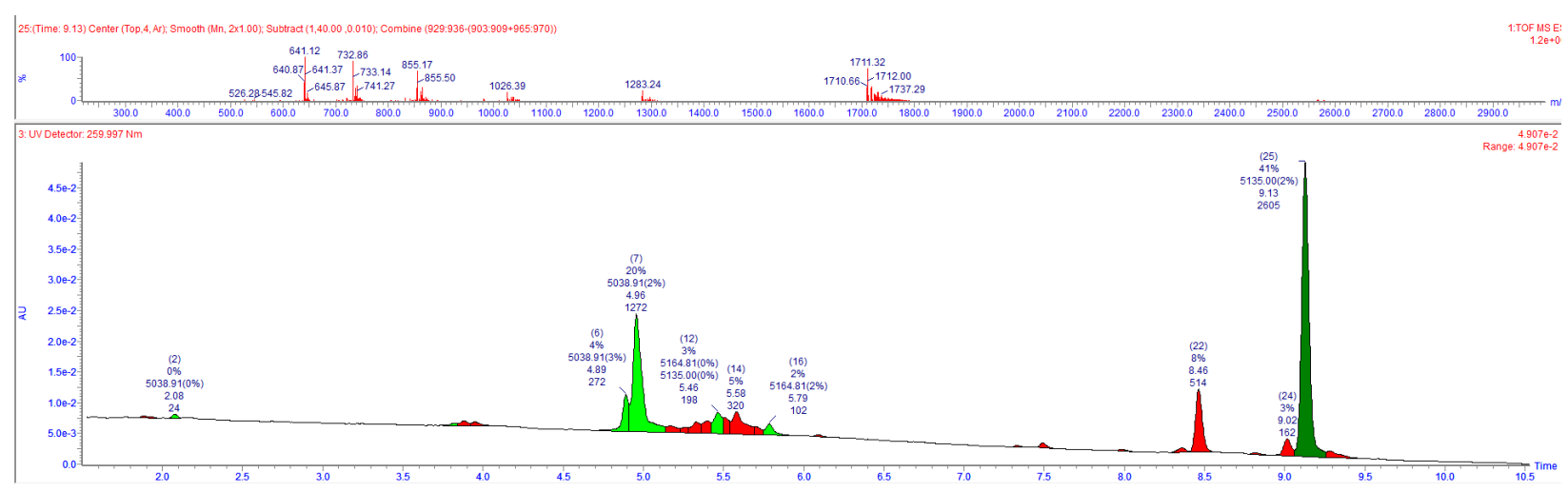




\section{LC Trace and Mass of 17 in tripliacate}

\section{Following General Procedure 5B with 1}

Yields: 78\%, 61\%, 67\%

Average Yield: $68.67 \%$

SD: 8.6

Exact mass: 5130.97

Triply charged masses [M-3]/3, caluculated: 1709.32; observed $1709.33,1709.33,1709.33$
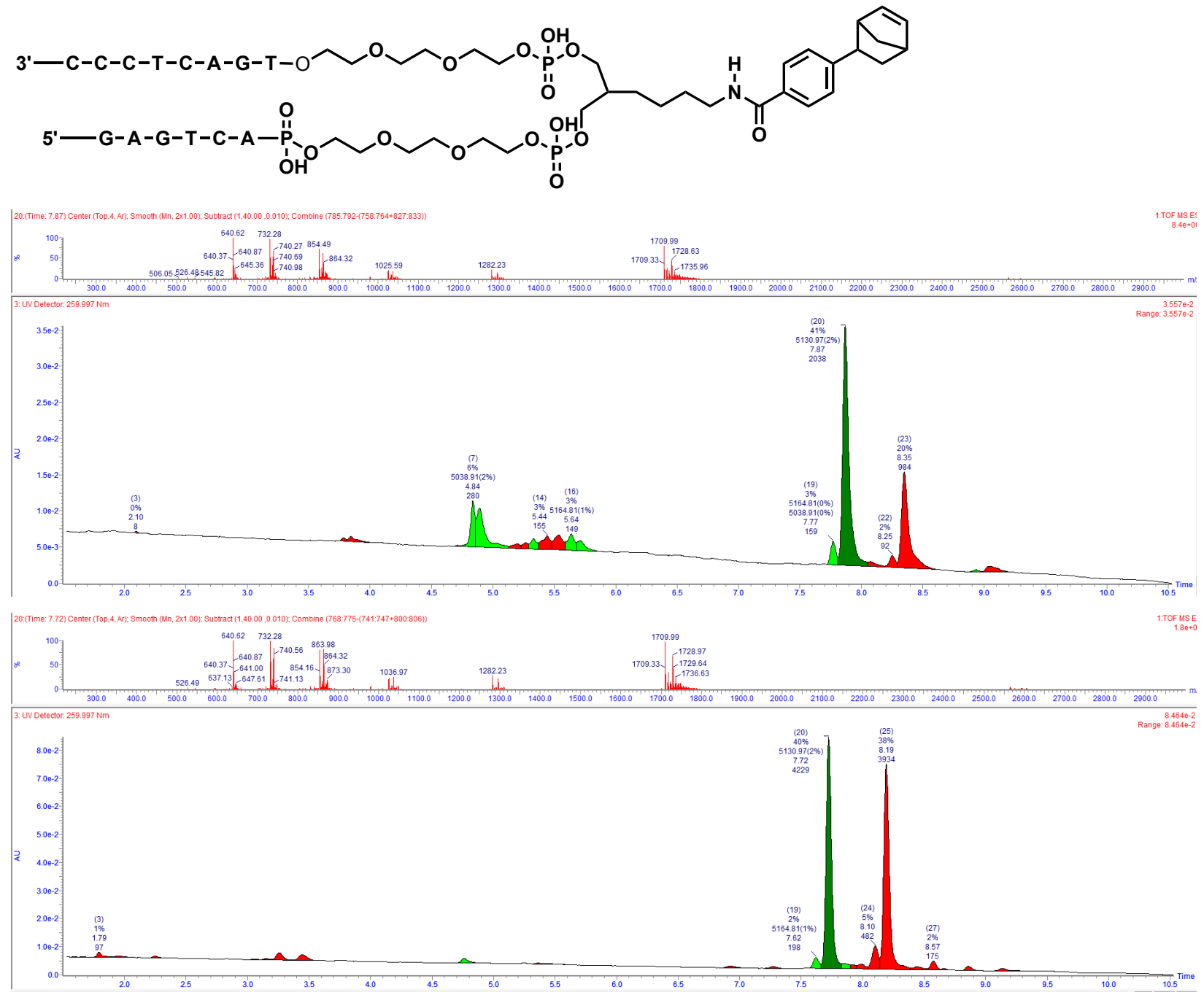


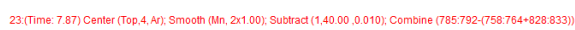

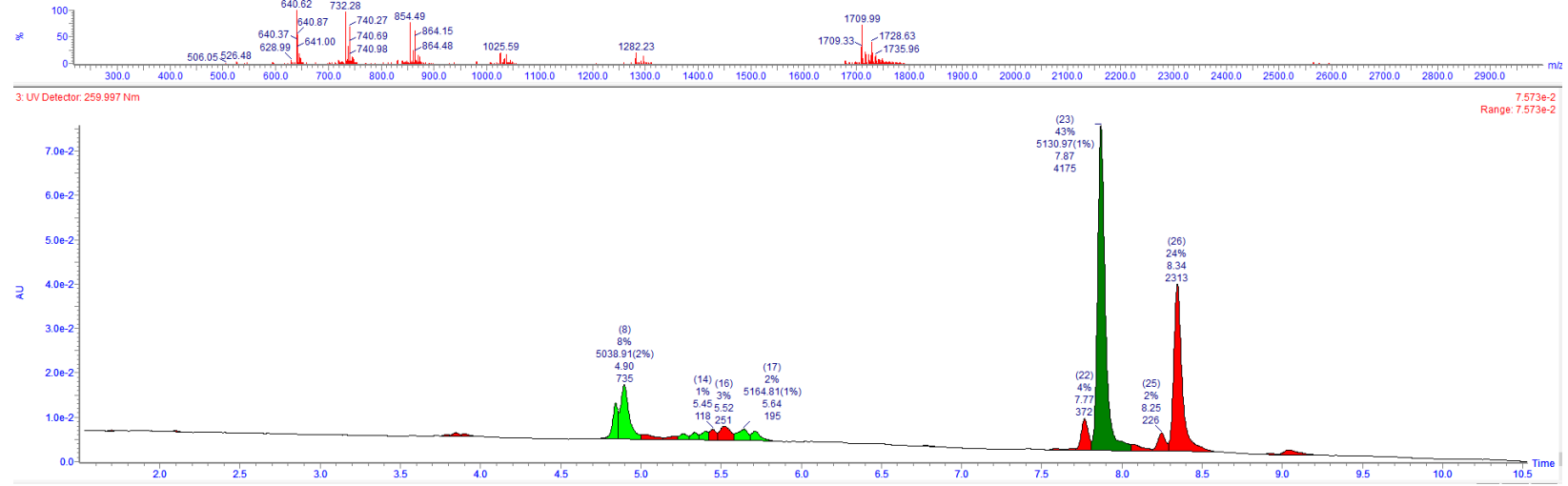




\section{LC Trace and Mass of 18 in tripliacate}

\section{Following General Procedure 5B with 1}

Yields: $64 \%, 57 \%, 60 \%$

Average Yield: $60.33 \%$

SD: 3.51

Exact mass: 5160.96

Triply charged masses [M-3]/3, caluculated: 1719.32; observed $1719.31,1719.31,1719.31$
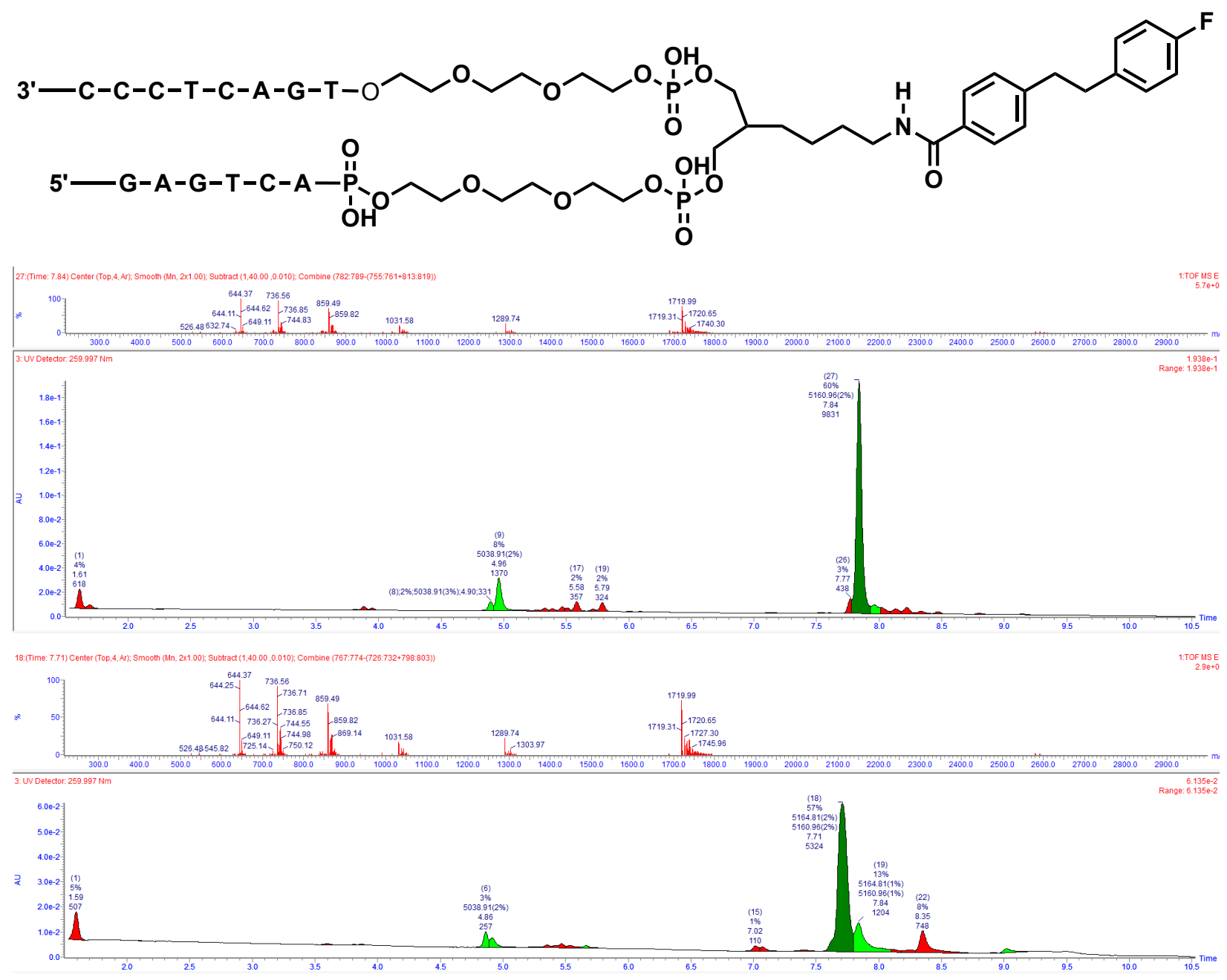


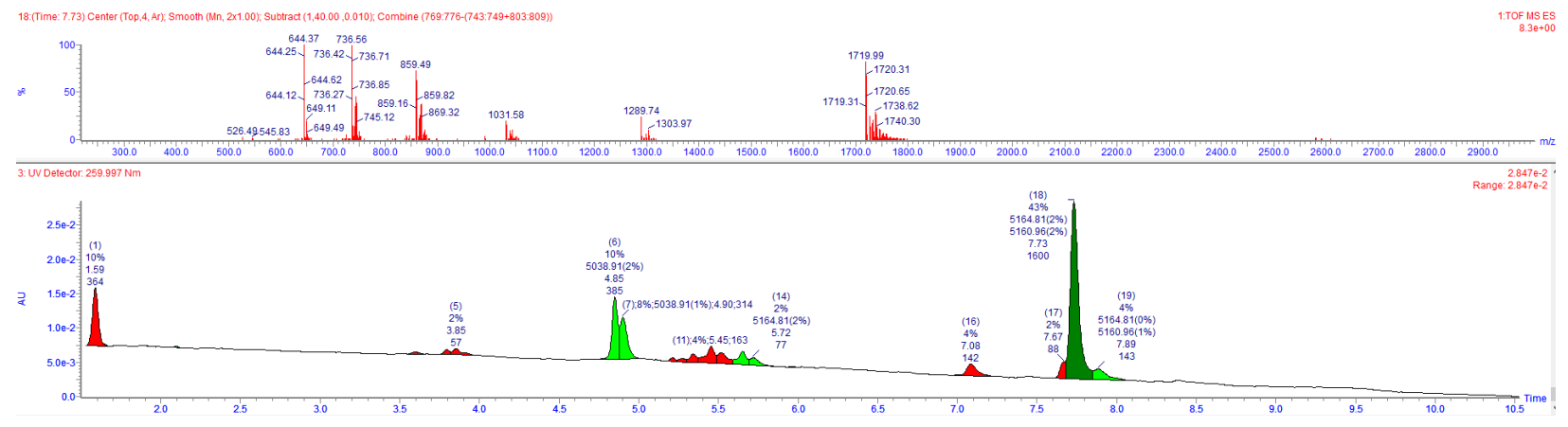

S78 


\section{LC Trace and Mass of 19 in tripliacate}

\section{Following General Procedure 5B with 1}

Yields: 67\%, 74\%, 79\%

Average Yield: $73.33 \%$

SD: 6.02

Exact mass: 5154.97

Triply charged masses [M-3]/3, caluculated: 1717.32; observed $1717.33,1717.31,1717.33$
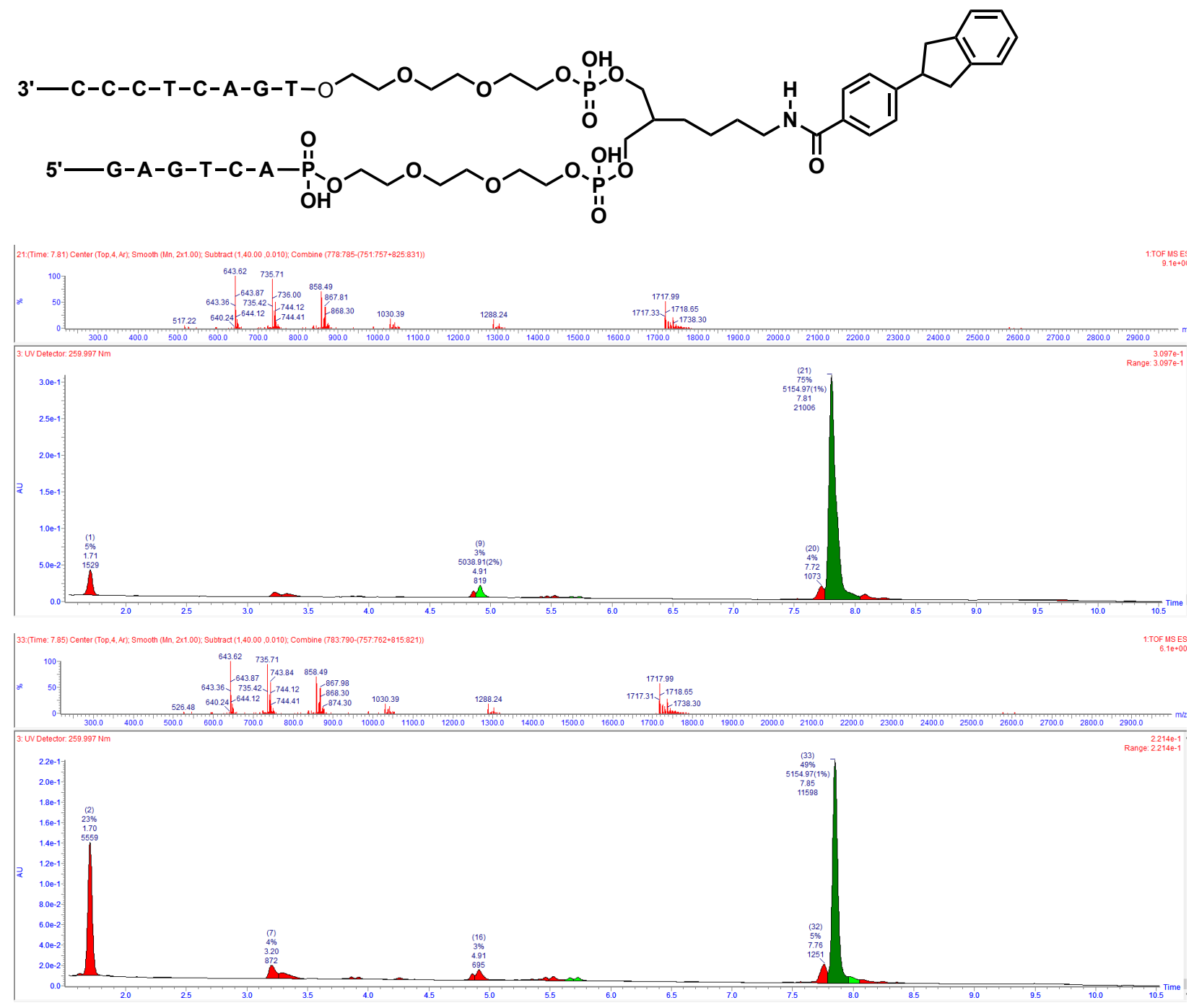


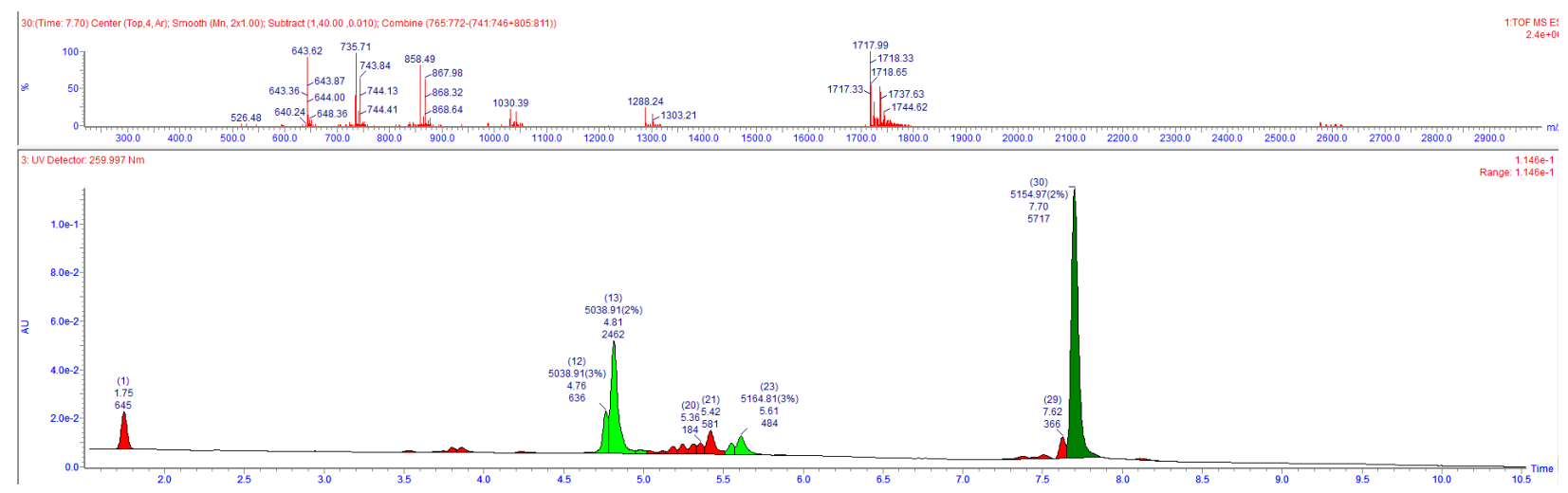




\section{LC Trace and Mass of 20}

Following General Procedure 5B with 5

Yields: $66 \%$

Exact mass: 5287.92

Triply charged masses [M-3]/3, caluculated: 1761.6; observed 1762.3,
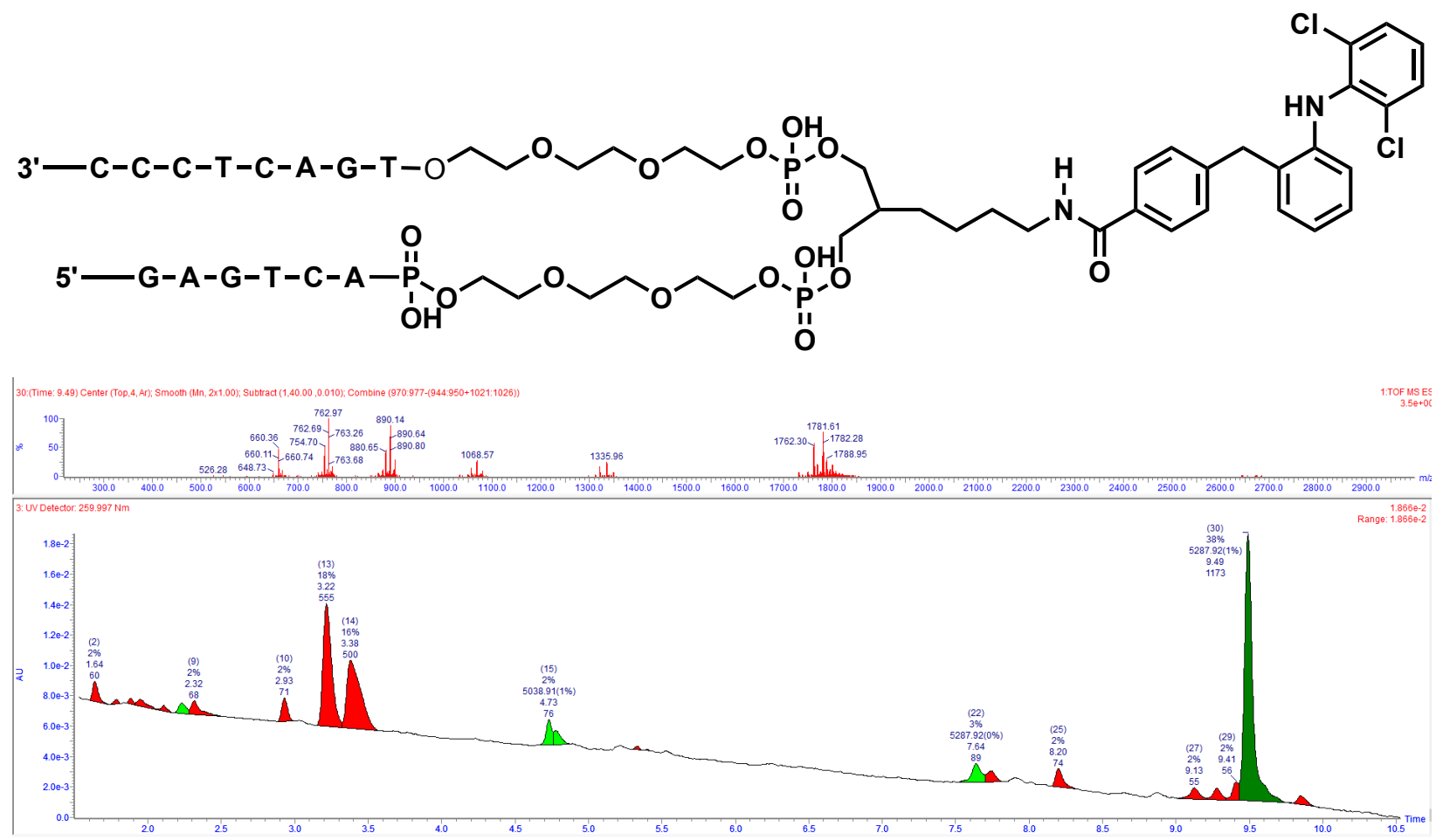


\section{LC Trace and Mass of 21 in tripliacate}

\section{Following General Procedure 5B with 1}

Yields: $81 \%, 82 \%, 72 \%$

Average Yield: $78.33 \%$

SDs: 5.5

Exact masses: 5194.00

Triply charged masses [M-3]/3, caluculated: 1730.33; observed $1730.33,1730.33,1730.33$
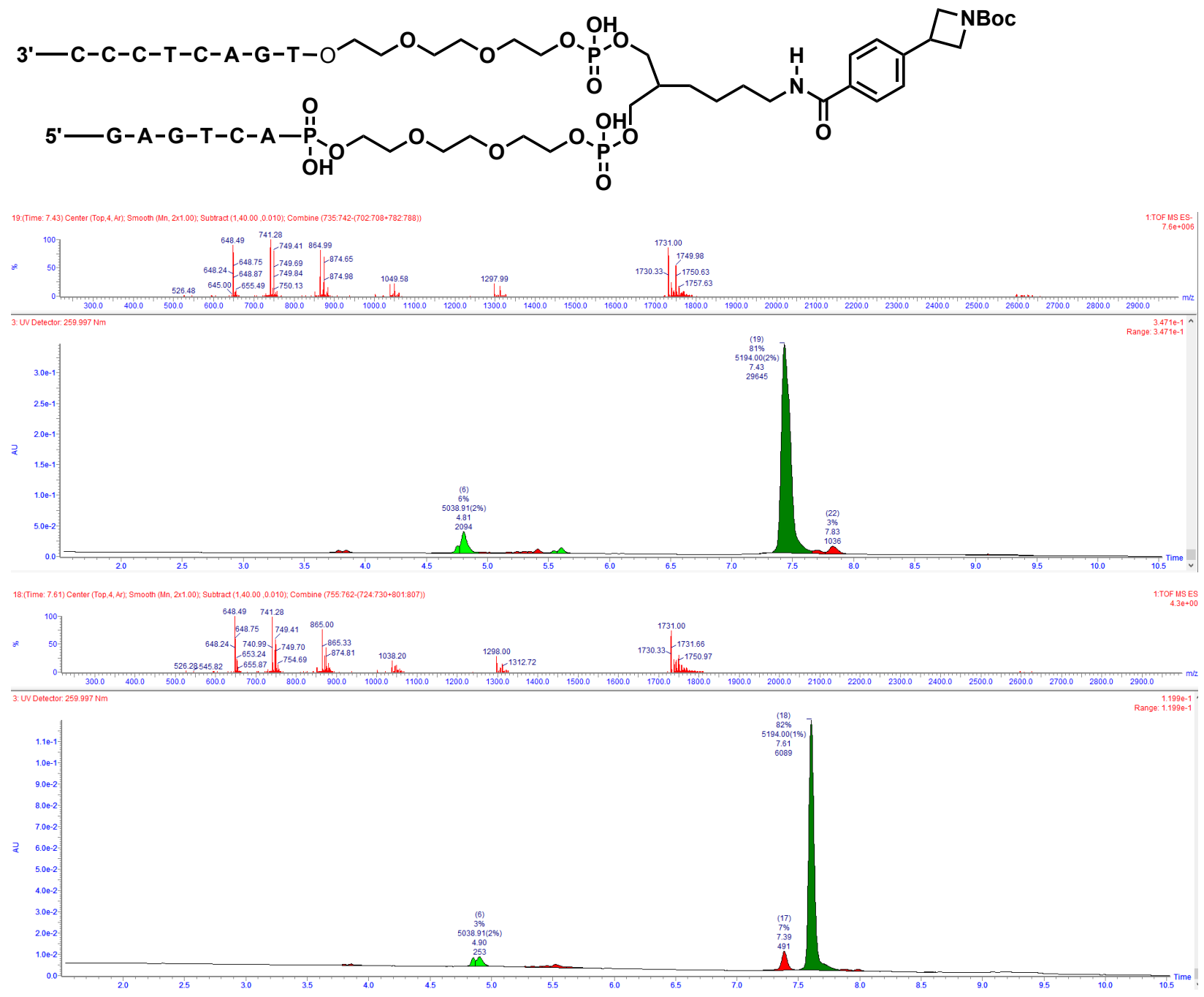


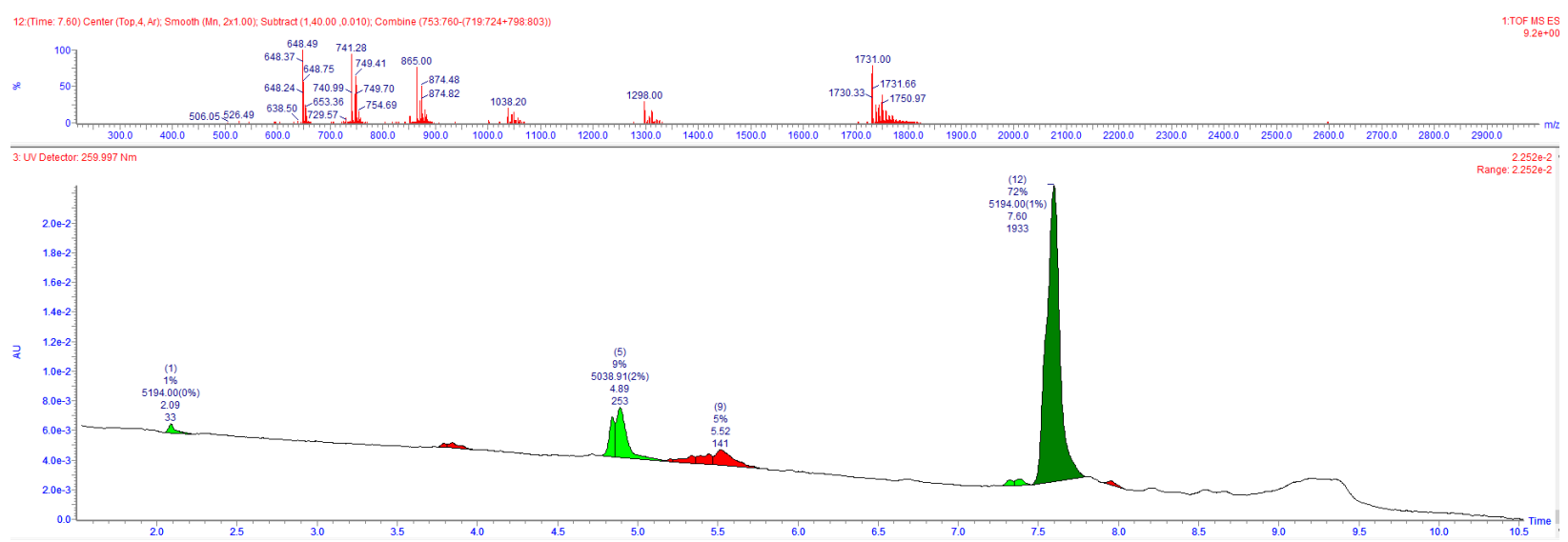




\section{LC Trace and Mass of 22}

Following General Procedure 5A with 1

Yield: $72 \%$

Exact mass: 5347.99

Triply charged mass [M-3]/3, caluculated: 1781.66; observed 1781.66
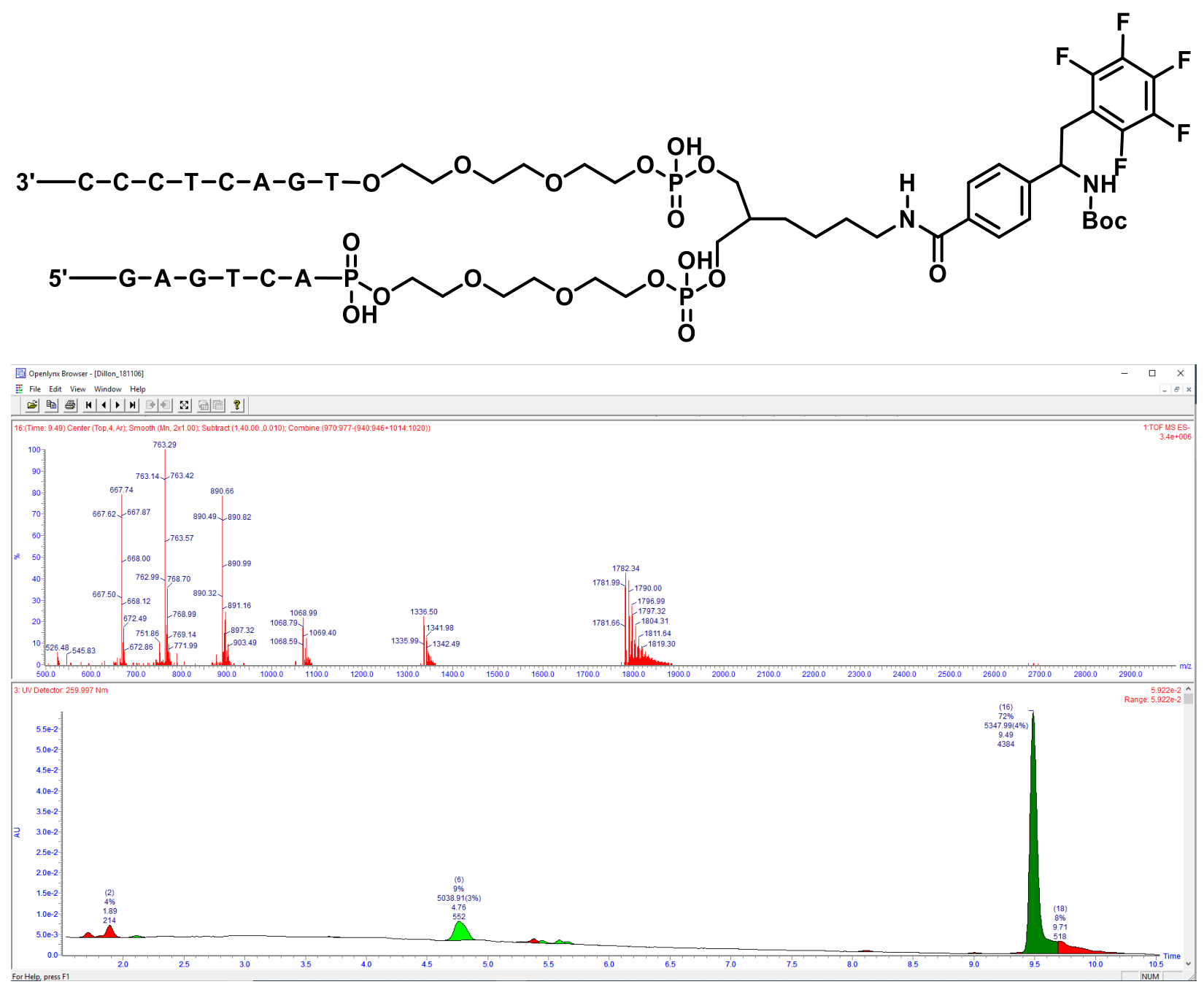


\section{LC Trace and Mass of 23}

Following General Procedure 5A with $\mathbf{1}$

Yield: 97\%

Exact mass: 5314.06

Triply charged mass [M-3]/3, caluculated: 1770.35 ; observed 1770.36
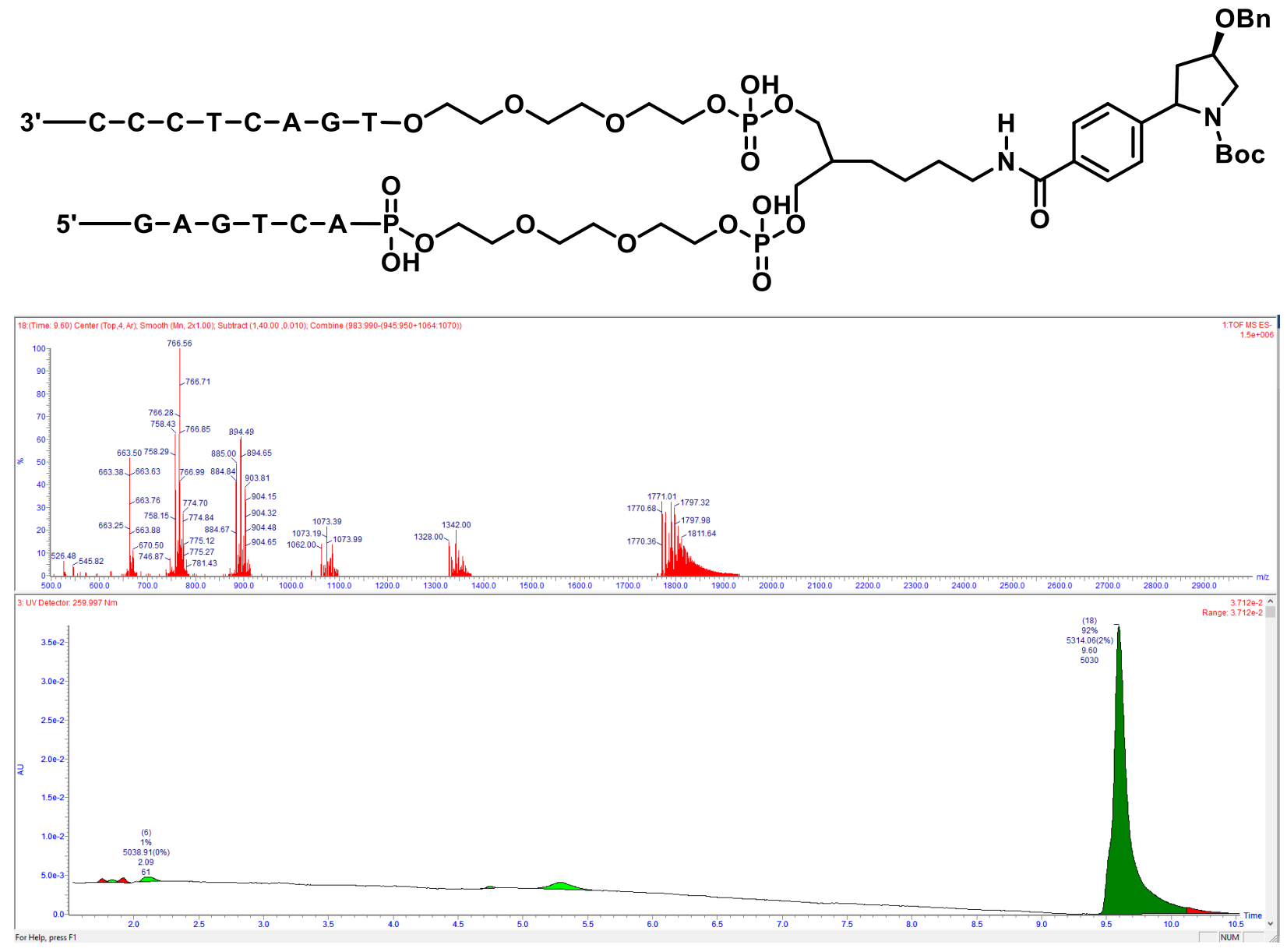


\section{LC Trace and Mass of 24}

Following General Procedure 5A with 1

Yield: 70\% (40\% Aldehyde and 30\% Alcohol)

Exact mass: 5172.95
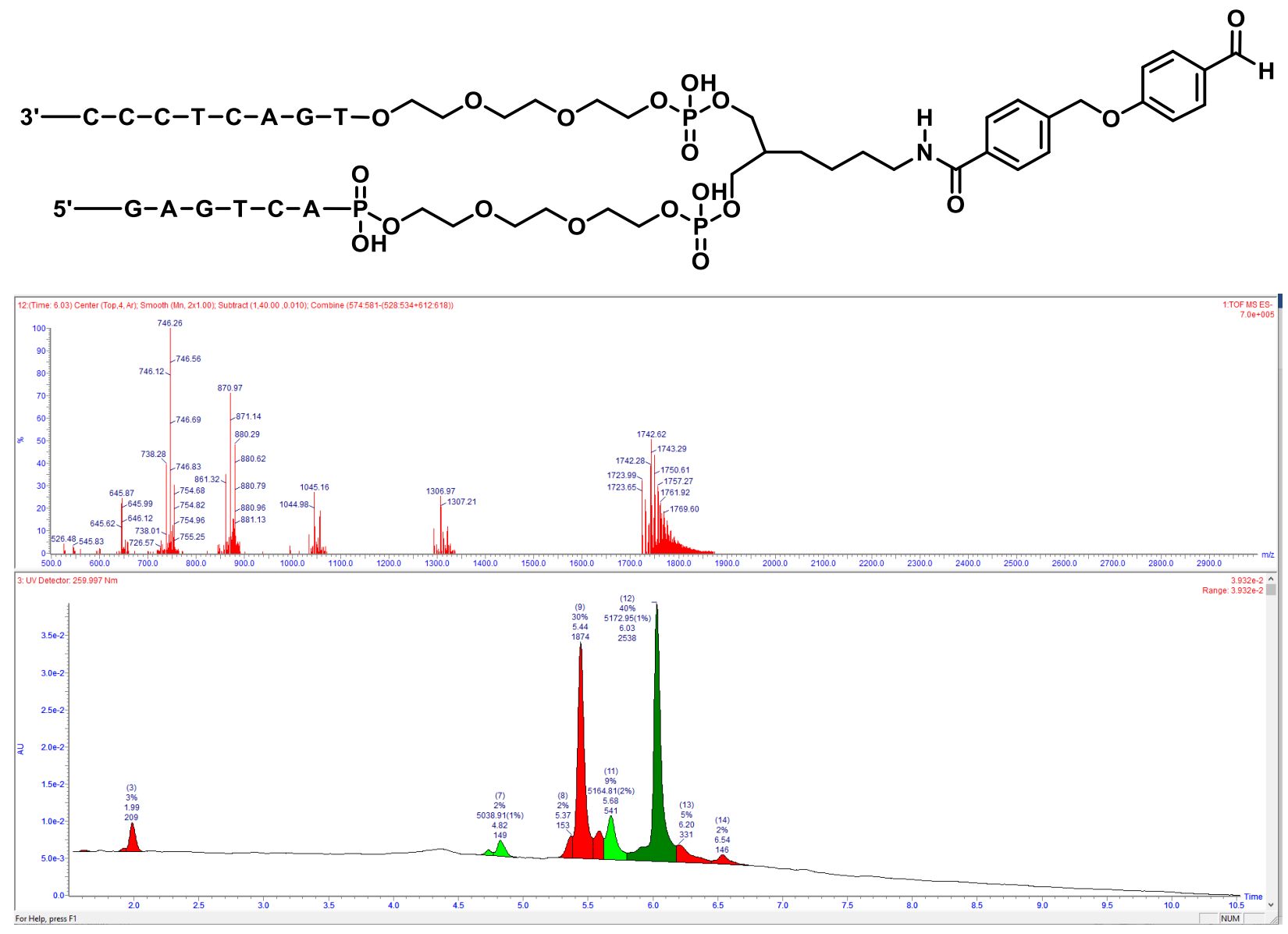

Triply charged mass [M-3]/3, caluculated: 1723.32; observed 1723.32

Yield: $30 \%$ of alcohol

Exact mass: 5174.96

Triply charged mass [M-3]/3, caluculated: 1723.98; observed 1723.99

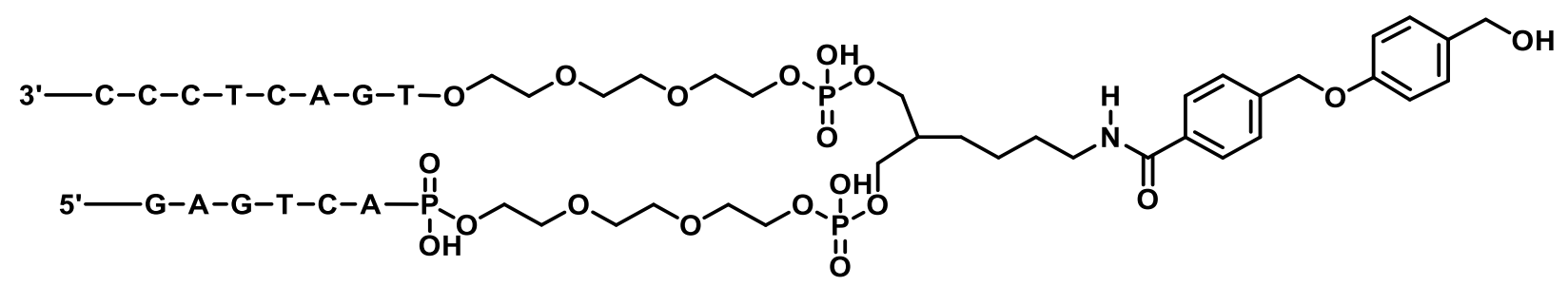




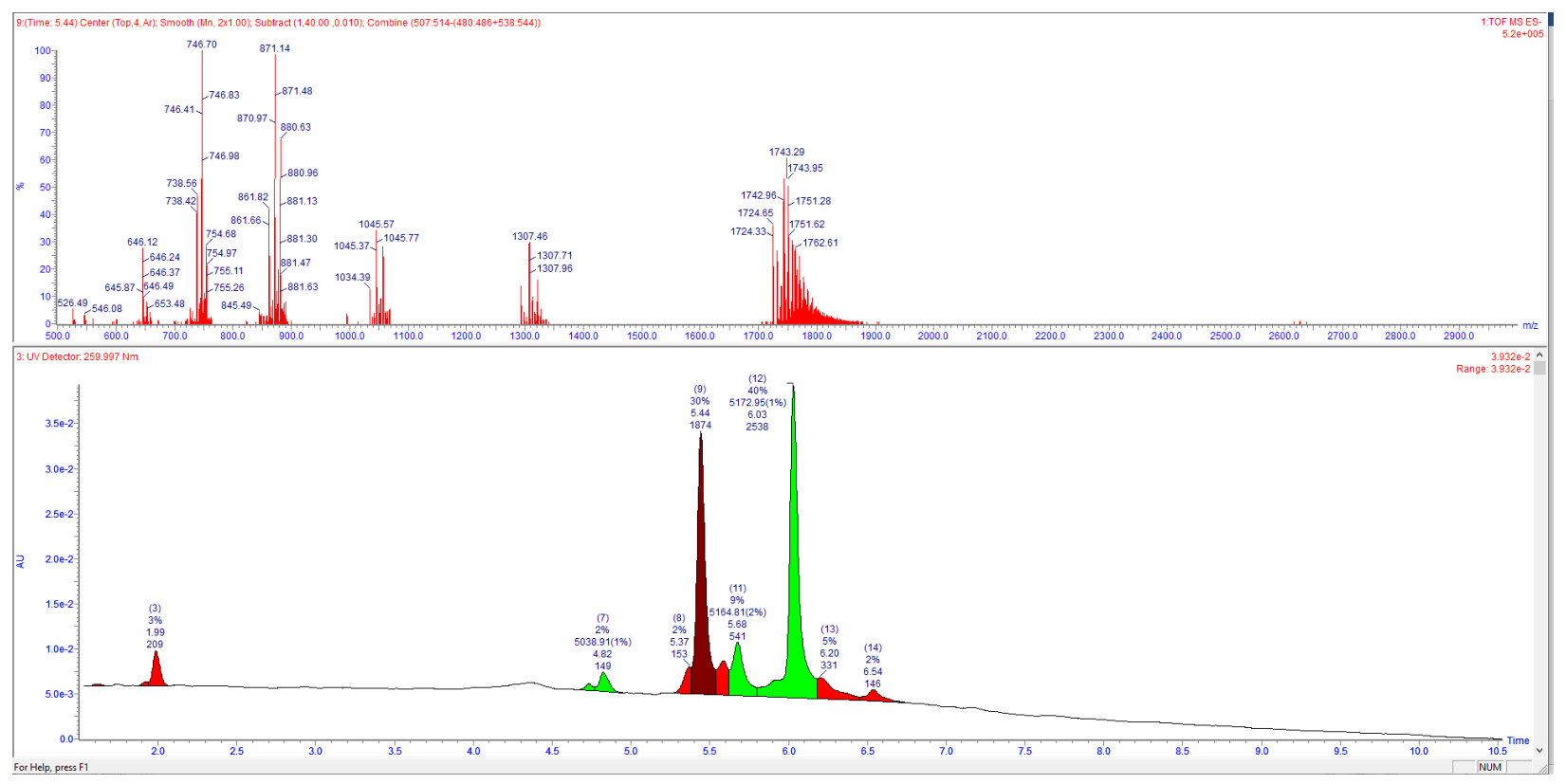




\section{LC Trace and Mass of 25}

Following General Procedure 5A with 1

Yield: $49 \%$

Exact mass: 5152.98

Triply charged mass [M-3]/3, caluculated: 1716.66; observed 1716.66
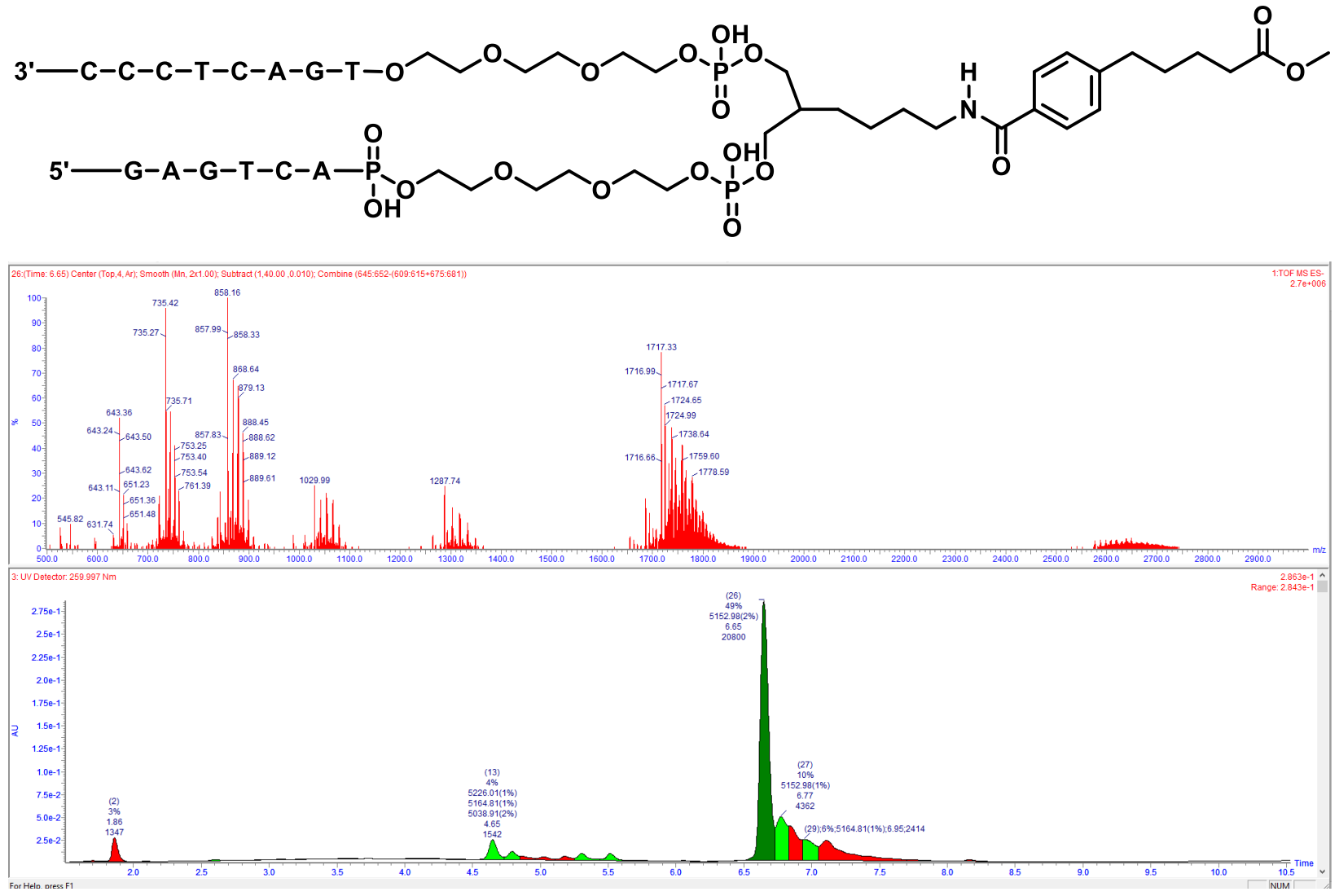
LC Trace and Mass of 26

Following General Procedure 5A with 1

Yield: $67 \%$

Exact mass: 5156.99

Triply charged mass [M-3]/3, calculated: 1718.00; observed 1717.99
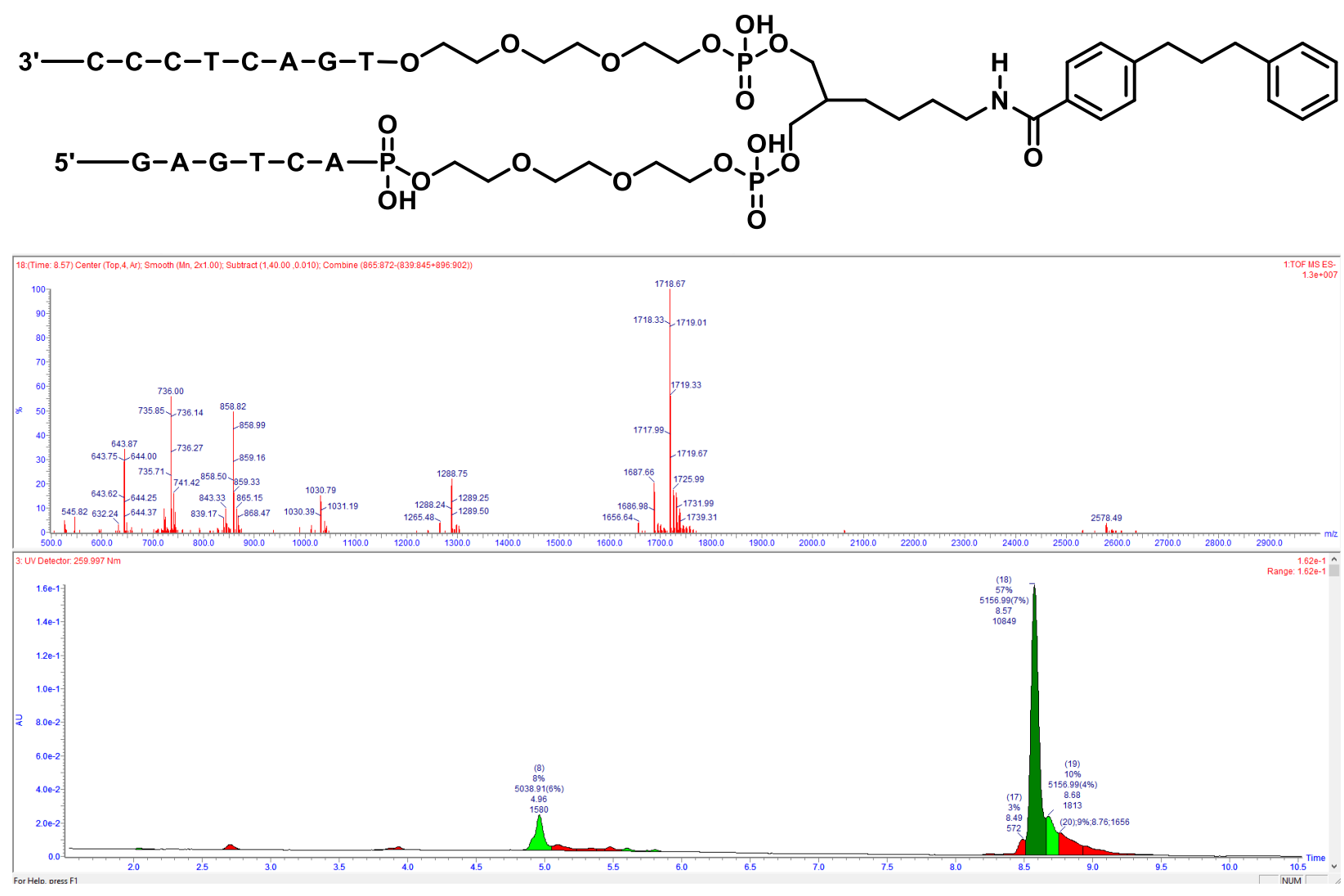

S 89 


\section{LC Trace and Mass of 27}

Following General Procedure 5A with 1

Yield: $60 \%$

Exact mass: 5092.96

Triply charged mass [M-3]/3, caluculated: 1696.65; observed 1696.65
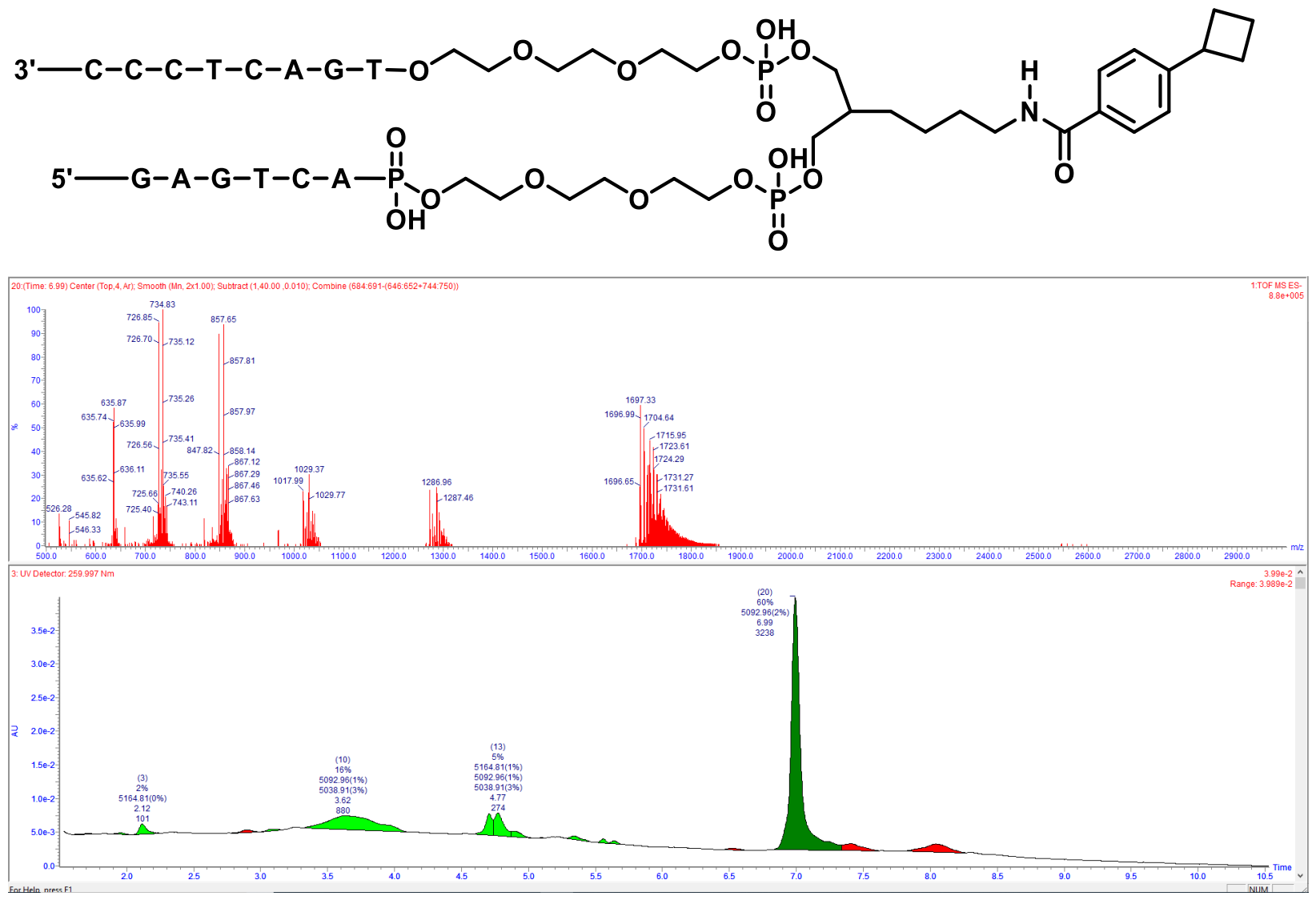


\section{LC Trace and Mass of 28}

Following General Procedure 5A with 1

Yield: $62 \%$

Exact mass: 5187.03

Triply charged mass [M-3]/3, caluculated: 1728.01; observed 1728.02
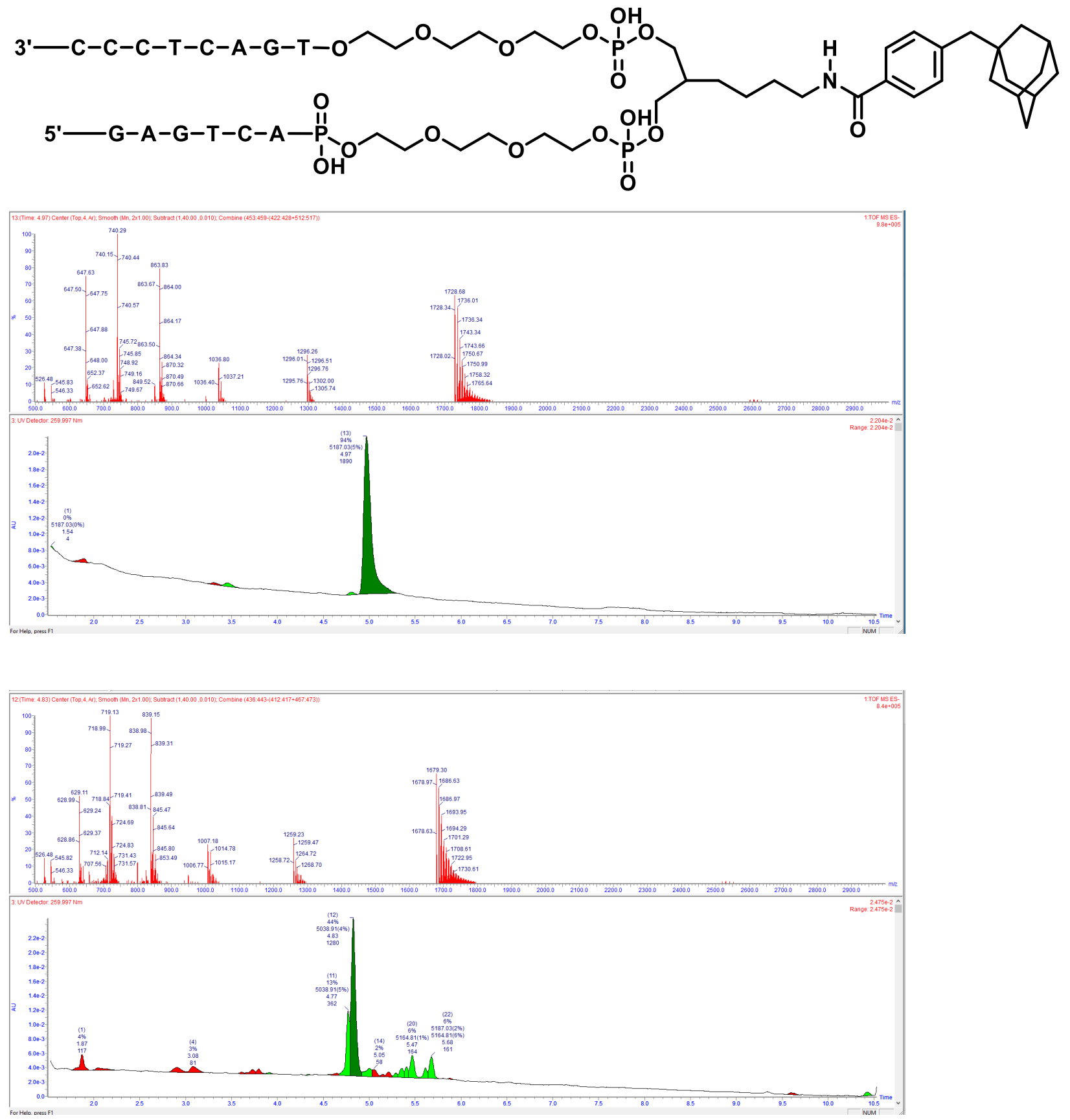


\section{LC Trace and Mass of 29}

Following General Procedure 5A with SI-7

Yield: $62 \%$

Exact mass: 5208.02

Triply charged mass [M-3]/3, caluculated: 1735.01; observed 1735.00
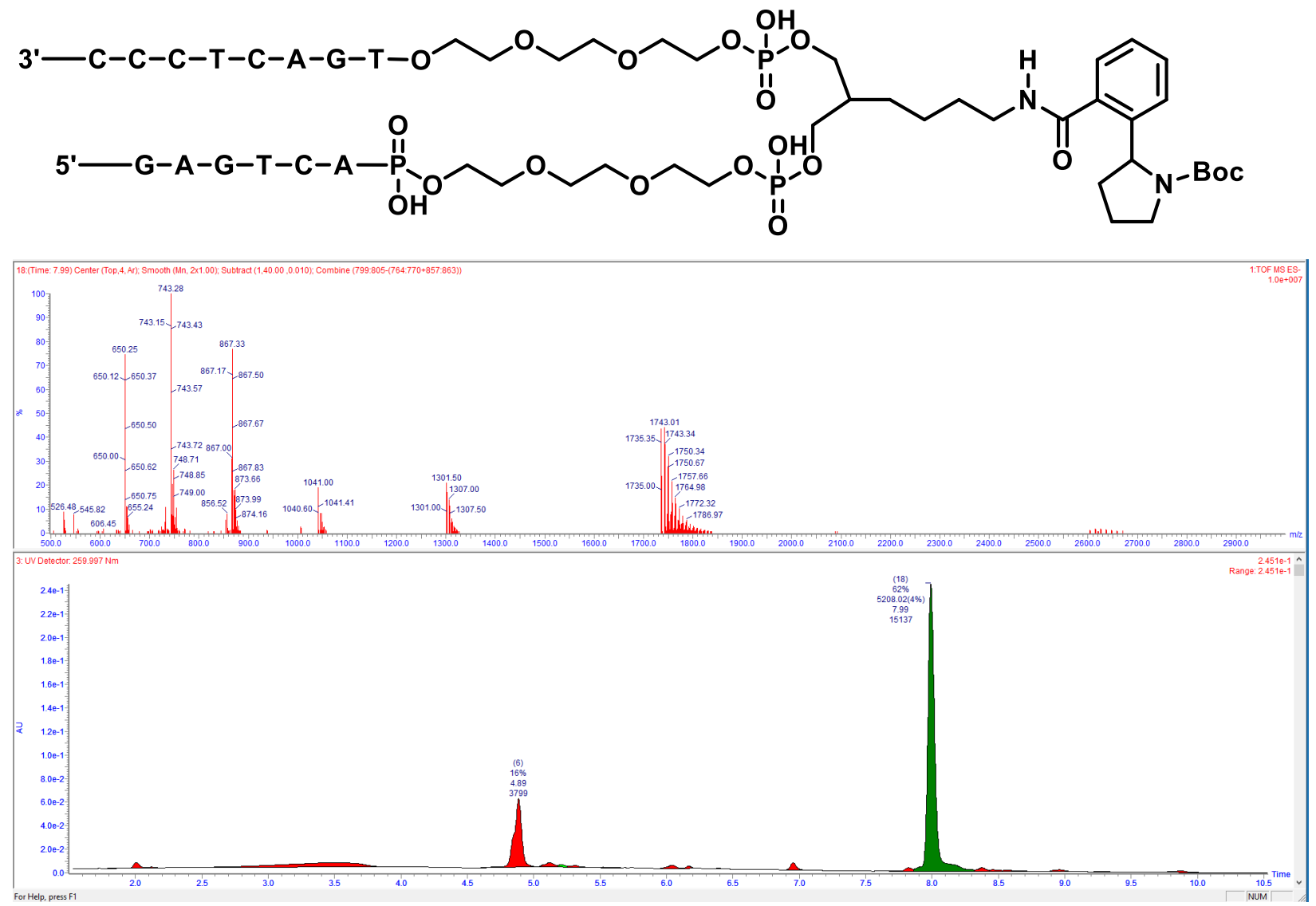


\section{LC Trace and Mass of 30}

Following General Procedure 5A with SI-6

Yield: 53\%

Exact mass: 5208.02

Triply charged mass [M-3]/3, caluculated: 1735.01; observed 1735.00
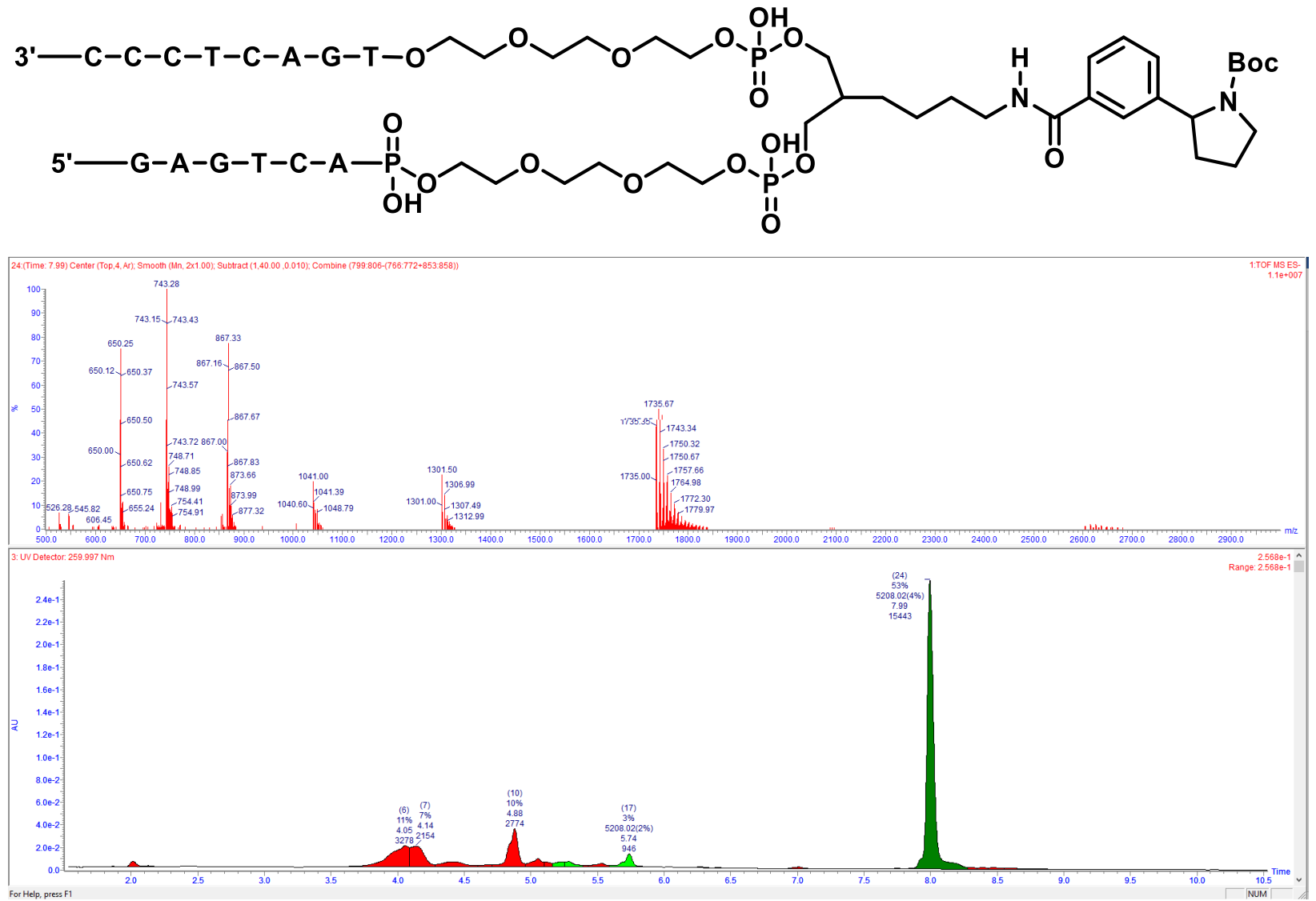


\section{LC Trace and Mass of 31 in tripliacate}

Following General Procedure 5B with SI-7

Yields: 48\%, 58\%, 74\%

Average Yield: $60 \%$

SD: 13

Exact mass: 5226.01

Triply charged masses [M-3]/3, caluculated: 1741.00; observed $1741.00,1741.00,1741.00$
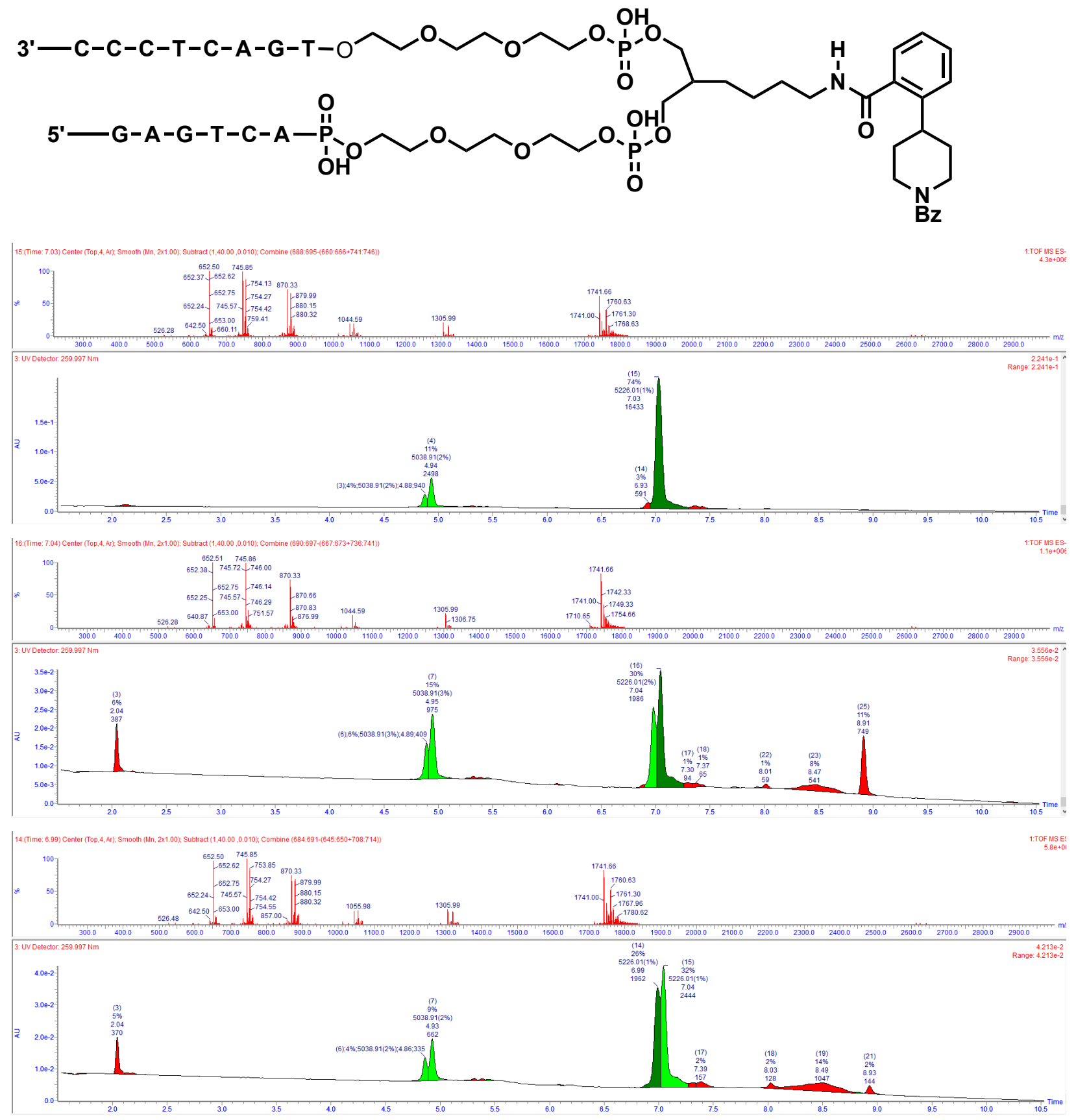
LC Trace and Mass of 32 in tripliacate

Following General Procedure 5A with SI-6

Yields: $82 \%, 81 \%, 85 \%$

Average Yield:82.6\%

SD: 2.0

Exact mass: 5226.01

Triply charged masses [M-3]/3, caluculated: 1741.00 ; observed $1741.00,1741.00,1741.00$
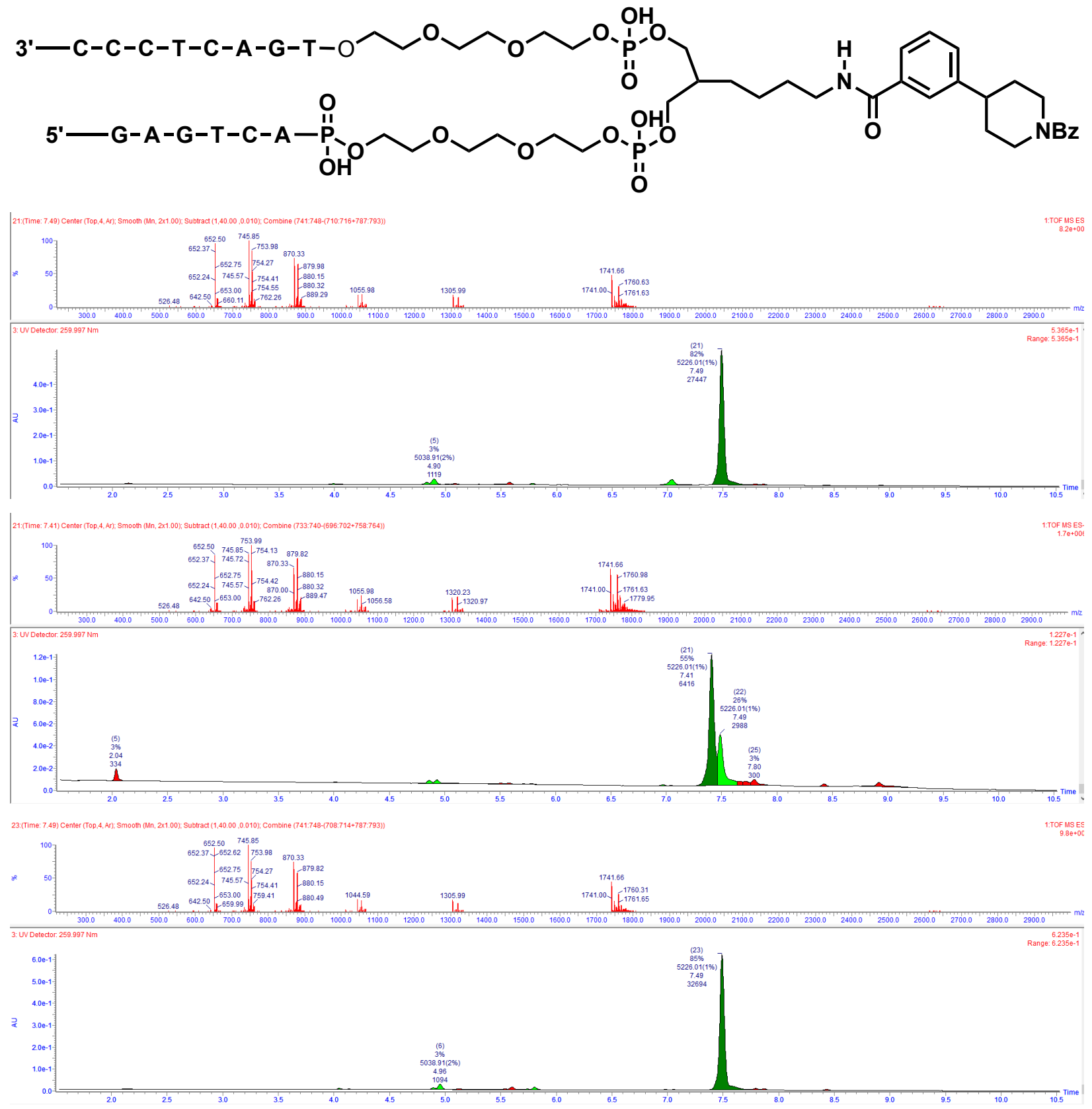


\section{LC Trace and Mass of 33 in tripliacate}

Following General Procedure 5B with 1

Yields: 39\%, 45\%, 44\%

Average Yield: $42.66 \%$

SD: 3.21

Exact mass5206.87

Triply charged masses [M-3]/3, caluculated: 1734.62; observed 1734.61, 1734.95, 1734.95
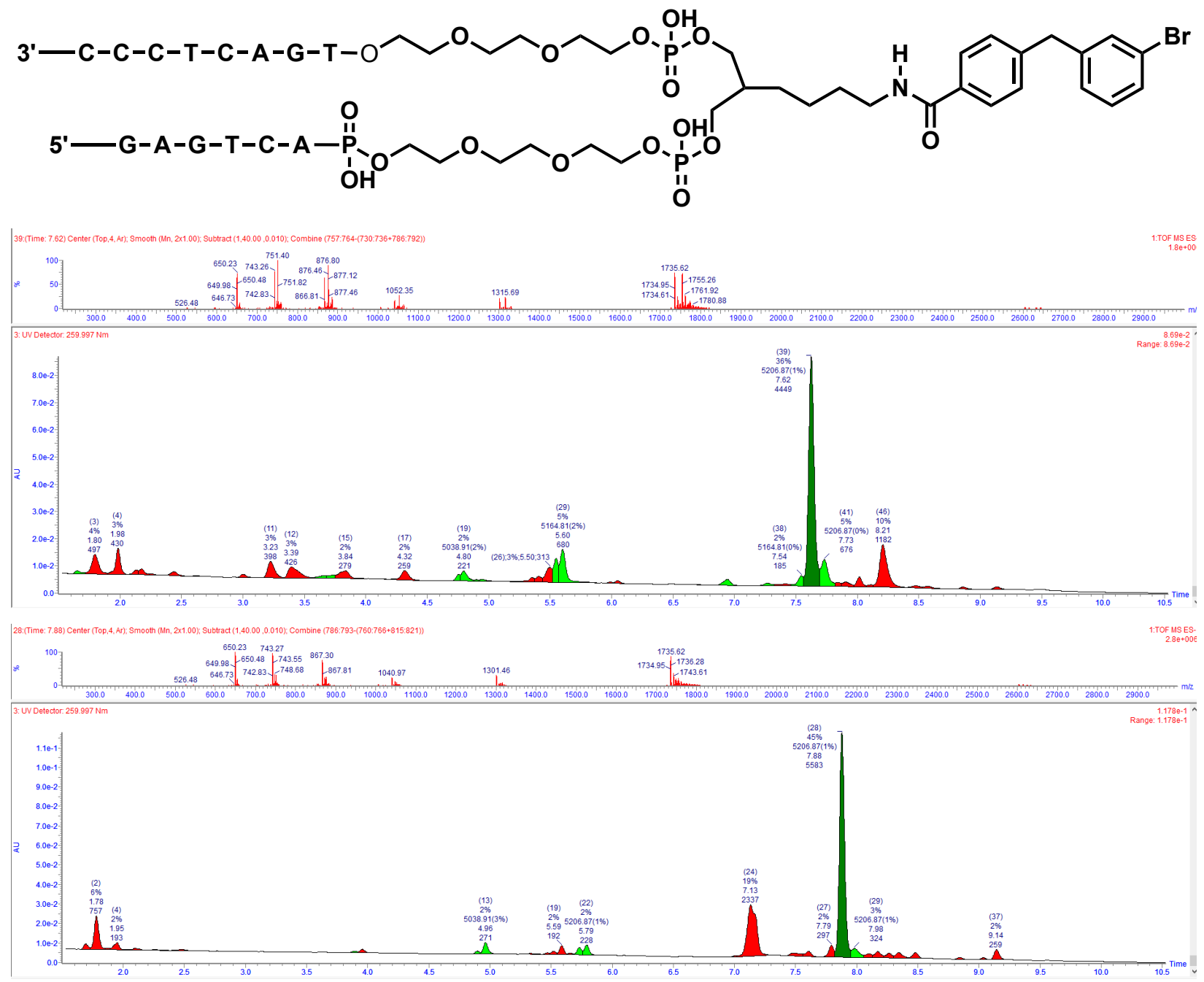


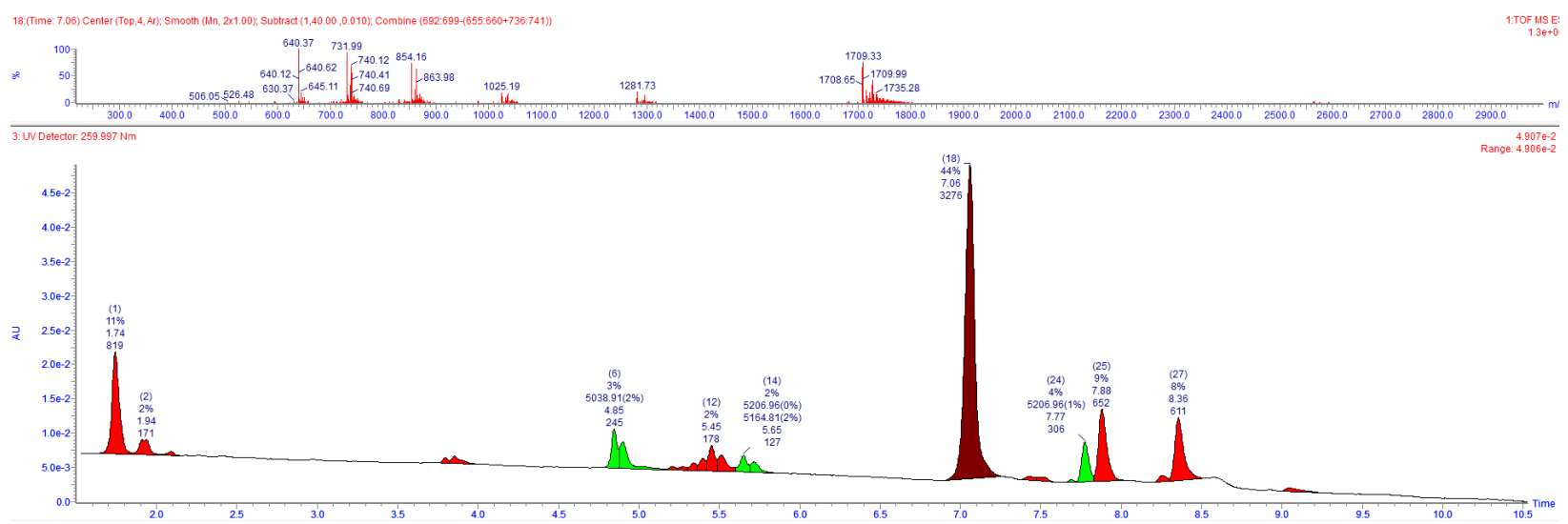




\section{LC Trace and Mass of 34 in tripliacate}

Following General Procedure 5B with SI-8

Yields: 77\%, 77\%, 91\%

Average Yield: 81.66\%

SD: 8.10

Exact mass: 5240.02

Triply charged masses [M-3]/3, caluculated: 1745.67; observed $1741.00,1741.00,1741.00$

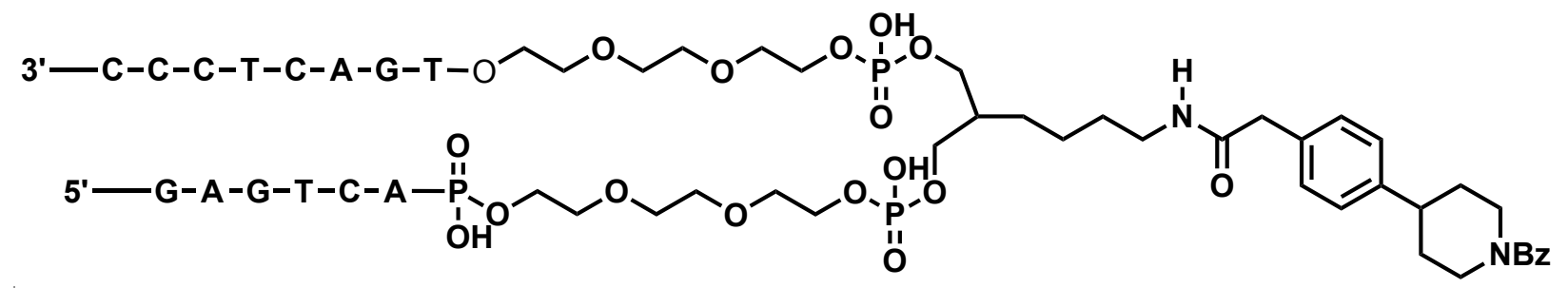

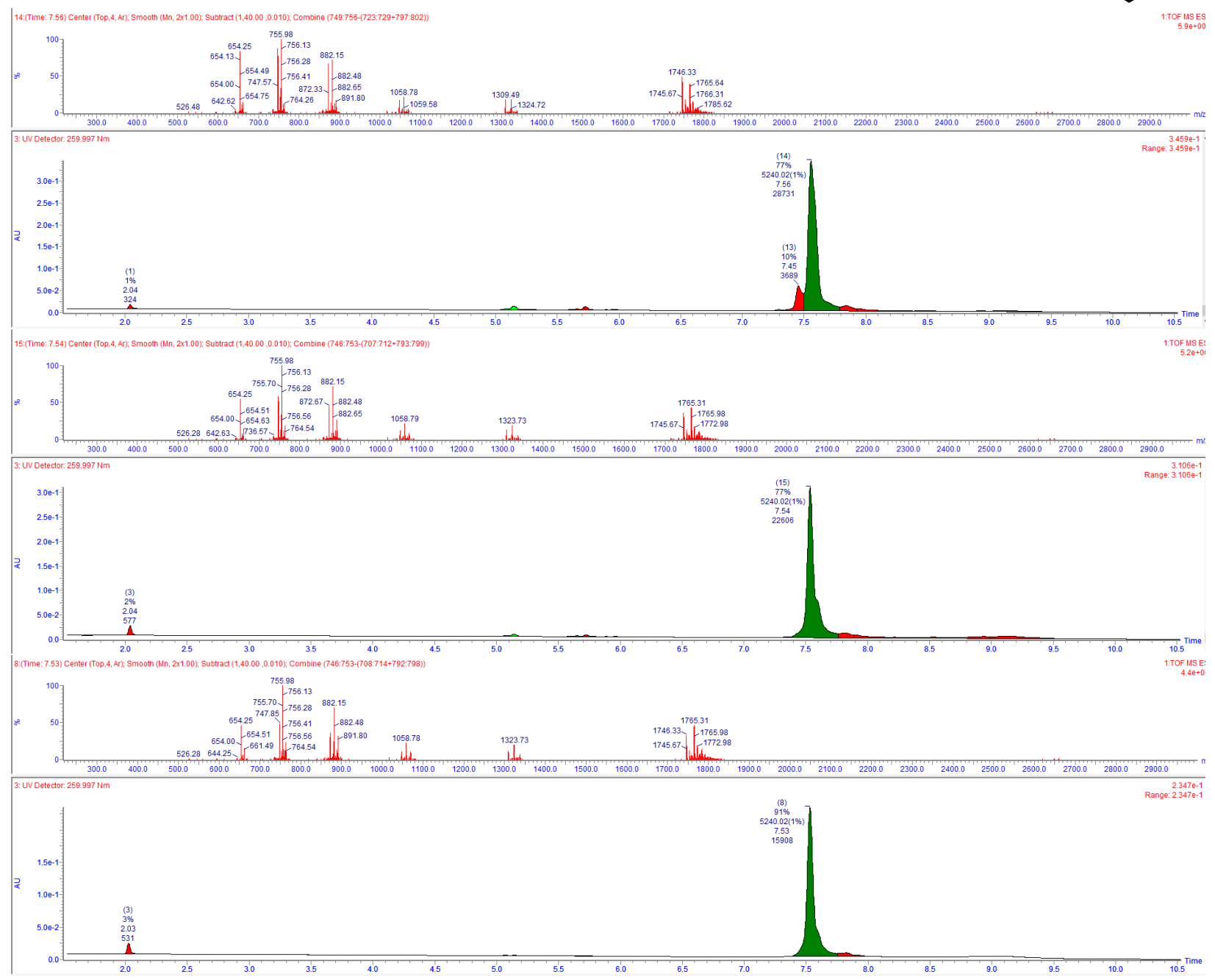




\section{LC Trace and Mass of 35}

Following General Procedure 5A with SI-5

Yield: $55 \%$

Exact mass: 5455.16

Triply charged mass [M-3]/3, caluculated: 1817.39; observed 1817.40
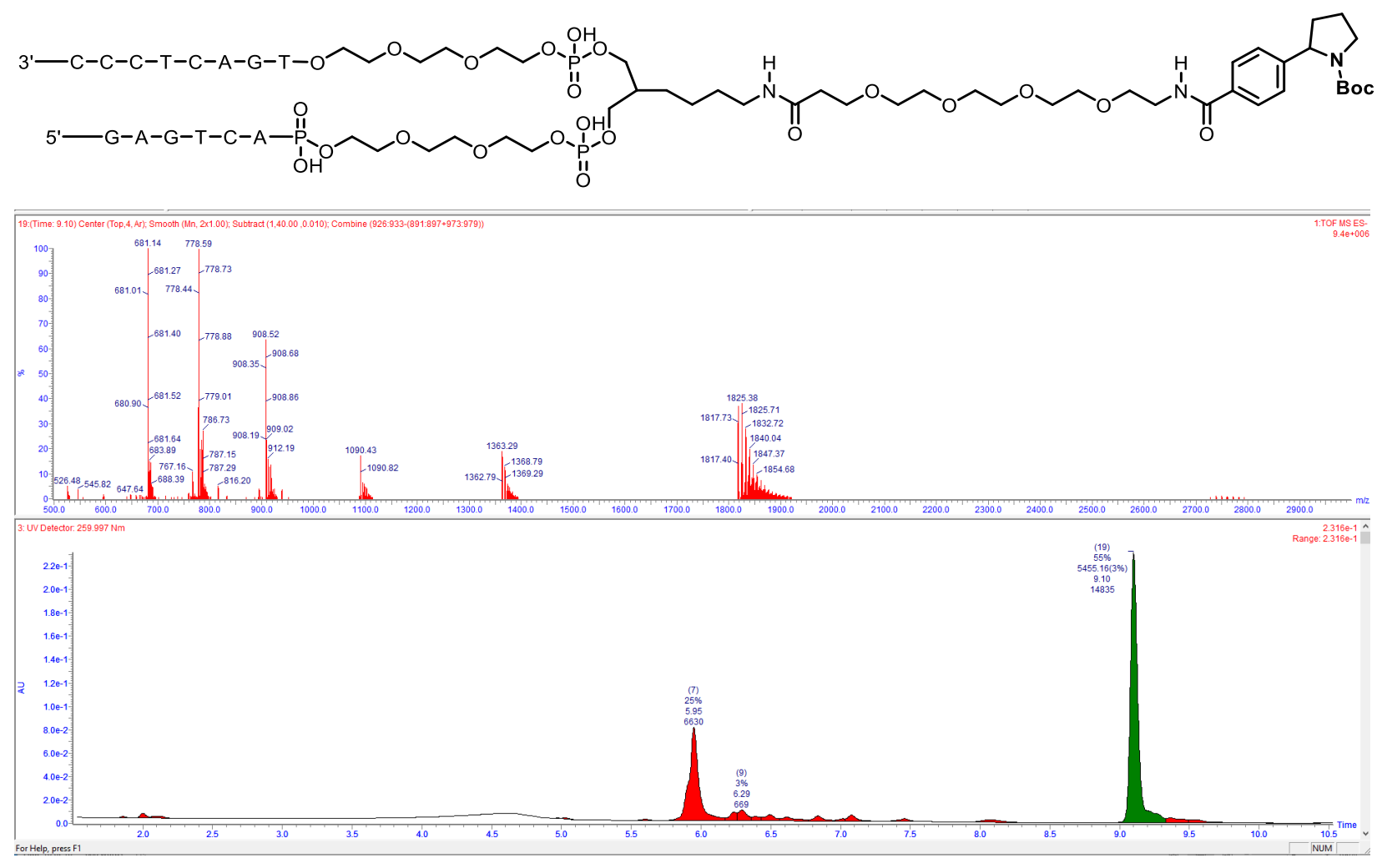


\section{LC Trace and Mass of 36}

Following General Procedure 5A with SI-11

Yield: $68 \%$

Exact mass: 5209.01

Triply charged mass [M-3]/3, caluculated: 1735.34; observed 1735.33
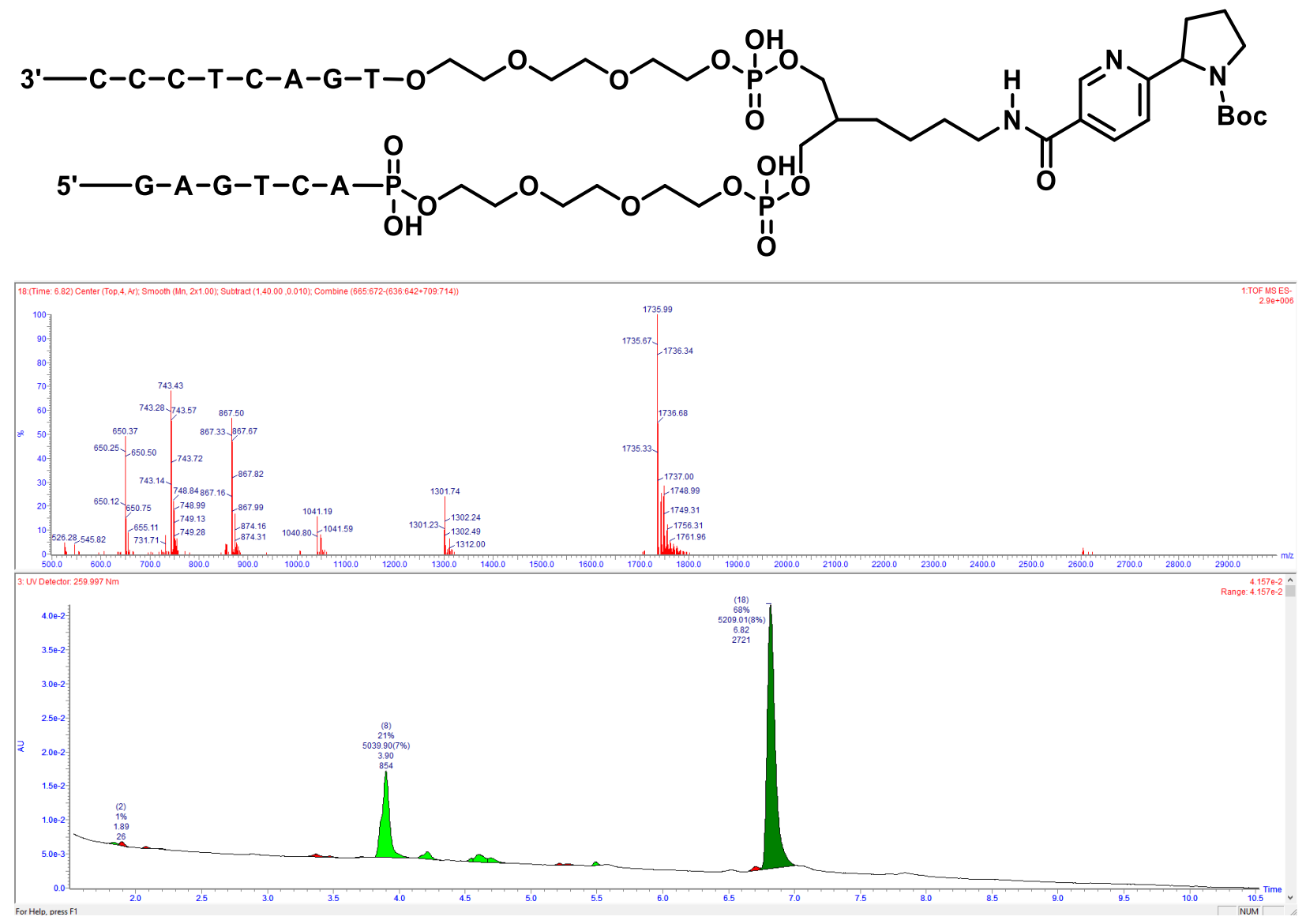


\section{LC Trace and Mass of 37 in tripliacate}

Following General Procedure 5B with SI-9

Yields: $46 \%, 42 \%, 48 \%$

Average Yield: $45.33 \%$

SD: 3.05

Exact mass: 5230.01

Triply charged masses [M-3]/3, caluculated: 1742.34; observed $1742.33,1742.33,1742.33$
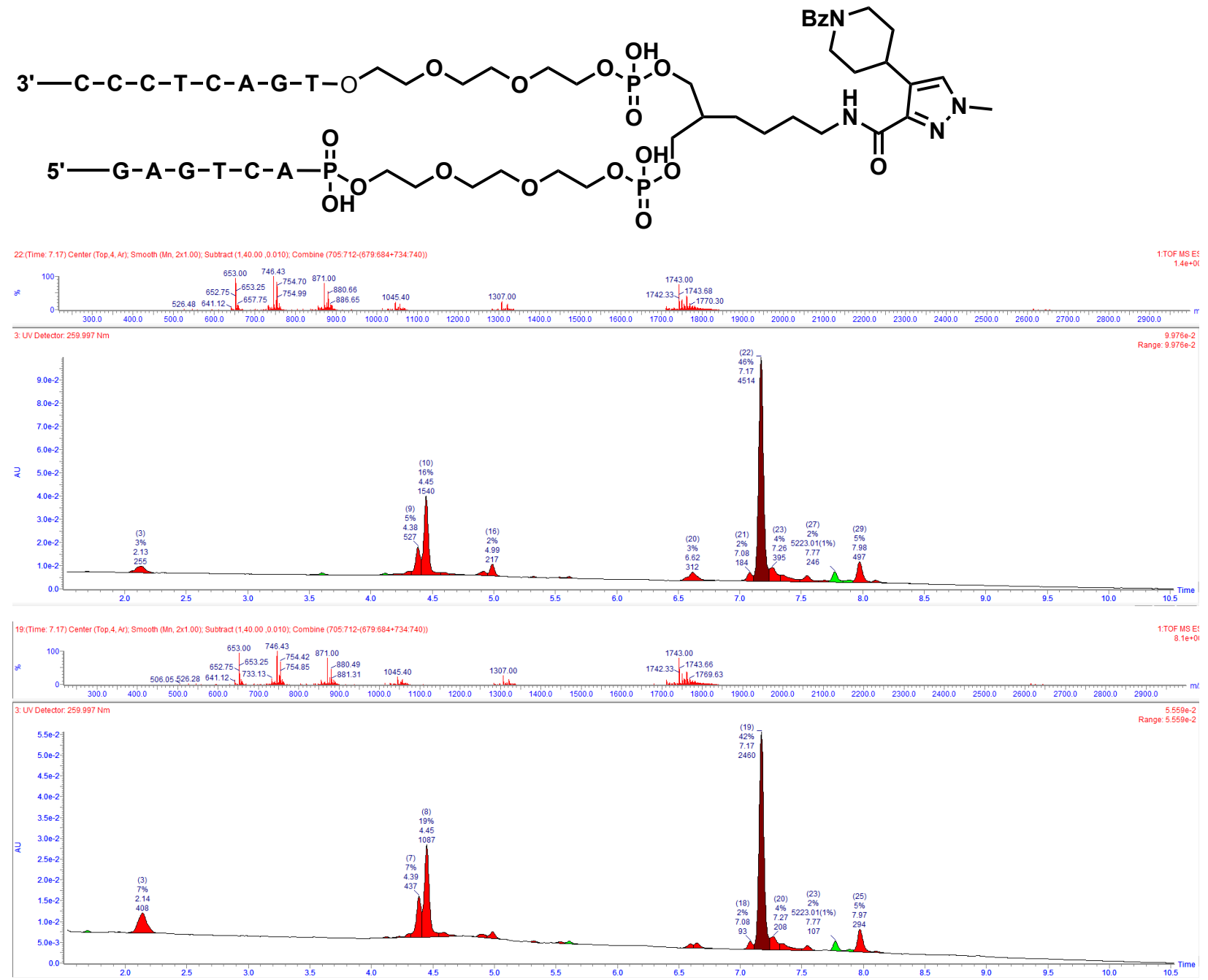


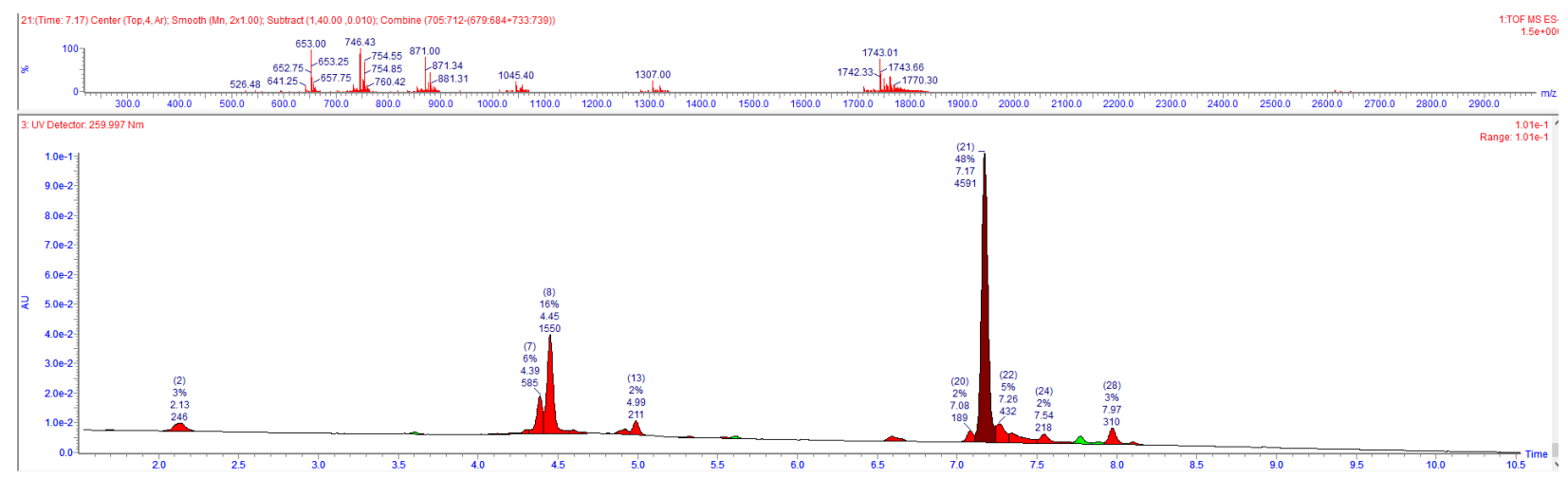




\section{LC Trace and Mass of 38 in tripliacate}

Following General Procedure 5B with SI-10

Yields: 46\%, 43\%, 46\%

Average Yield: $43 \%$

SD: 3

Exact mass: 5230.01

Triply charged masses [M-3]/3, caluculated: 1742.34; observed $1742.33,1742.33,1742.33$
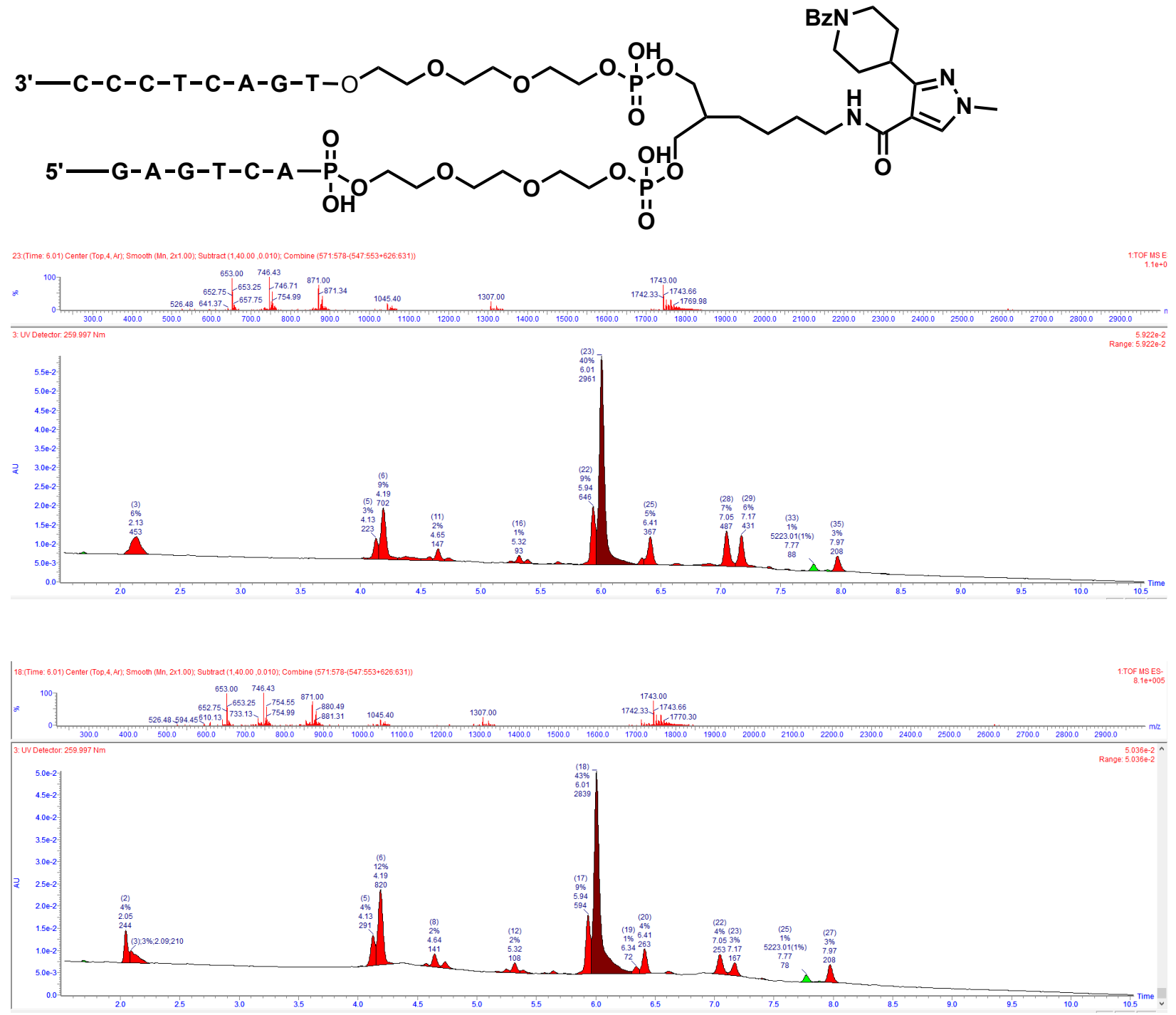


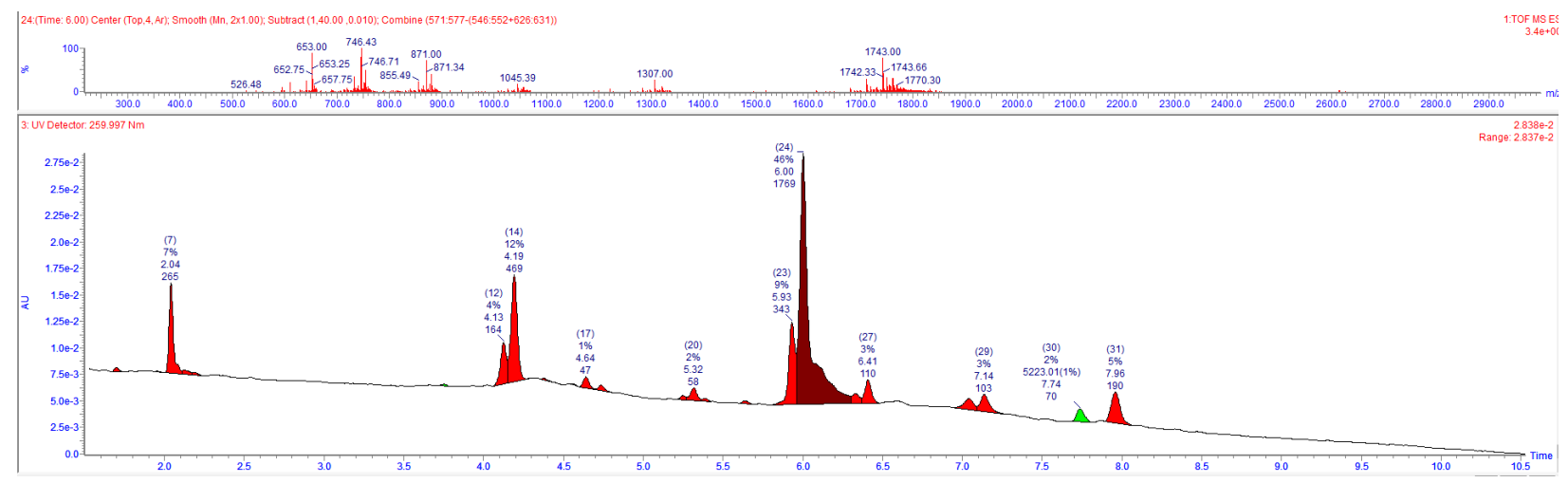




\section{LC Trace and Mass of 39}

Following General Procedure 5A with SI-4 and $250 \mathrm{mM} \mathrm{NaI}$.

Yield: $74 \%$

Exact mass: 5226.01

Triply charged mass [M-3]/3, caluculated: 1741.00; observed 1741.01
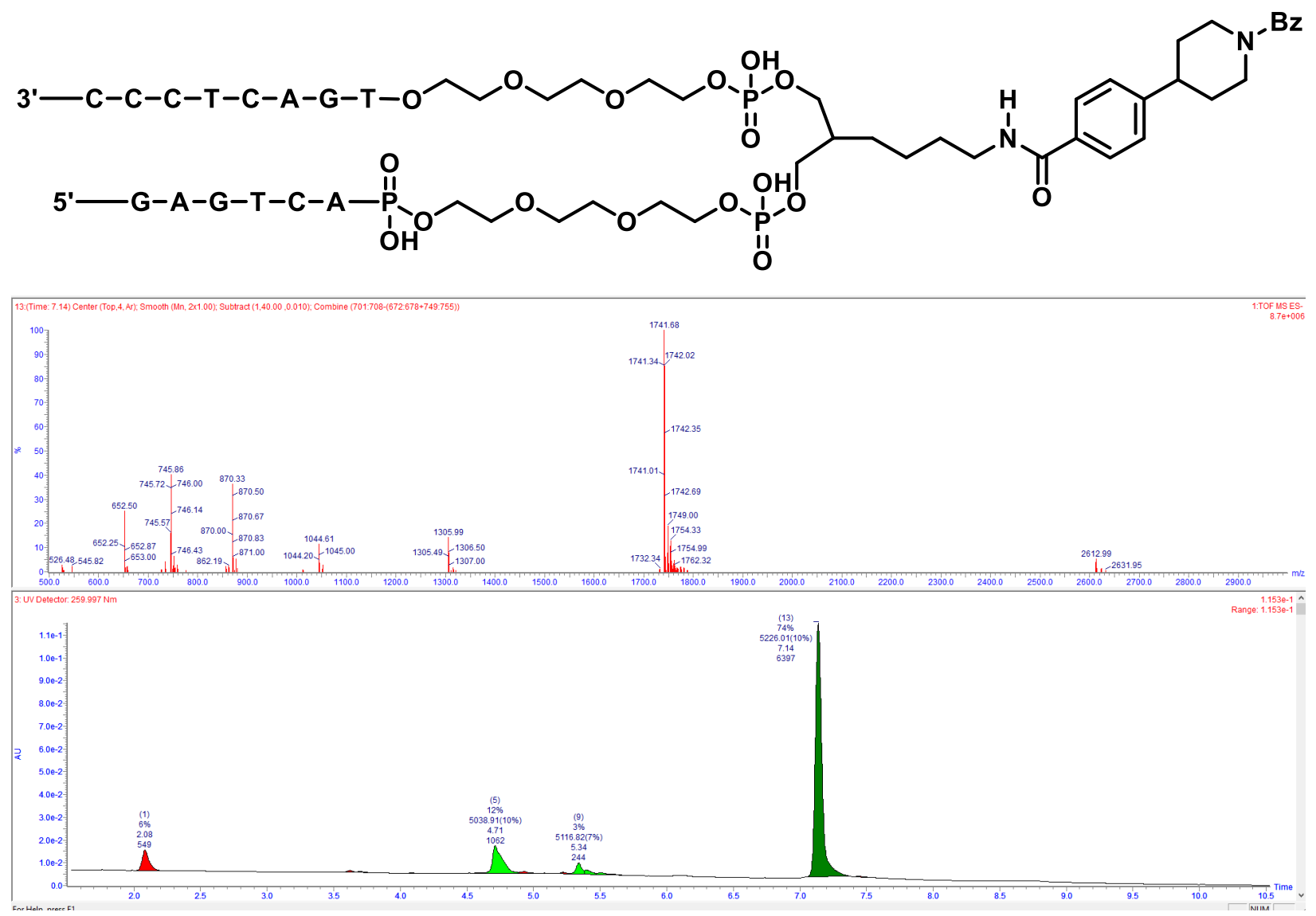


\section{LC Trace and Mass of 39 in tripliacate}

Following General Procedure 5B with SI-4 and $250 \mathrm{mM} \mathrm{NaI}$.

Yields: $77 \%, 86 \%, 81 \%$

Average Yield: $81.33 \%$

SD: 4.5

Exact mass: 5226.01

Triply charged masses [M-3]/3, caluculated: 1741.00; observed 1741.00, 1741.00, 1741.00
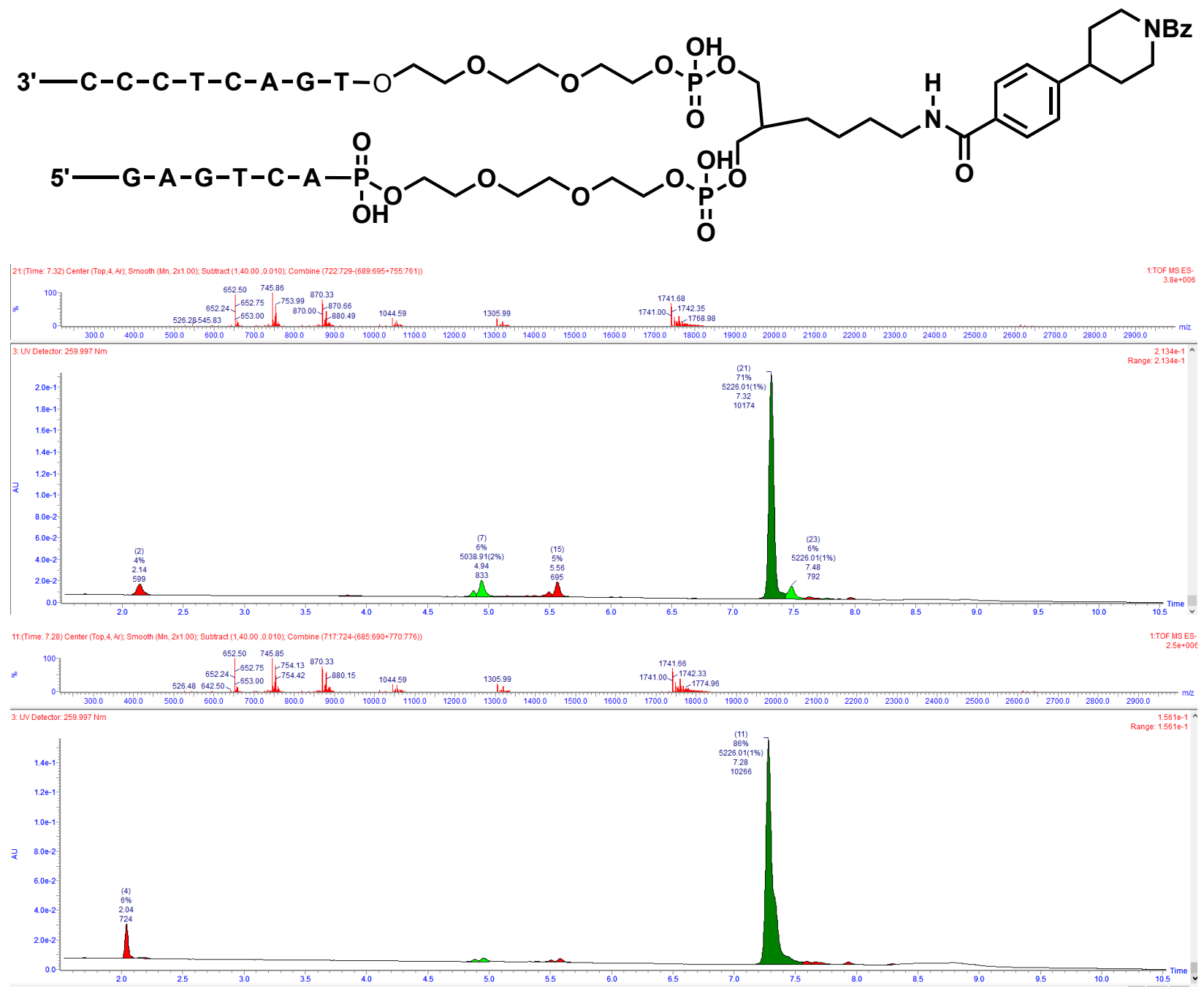


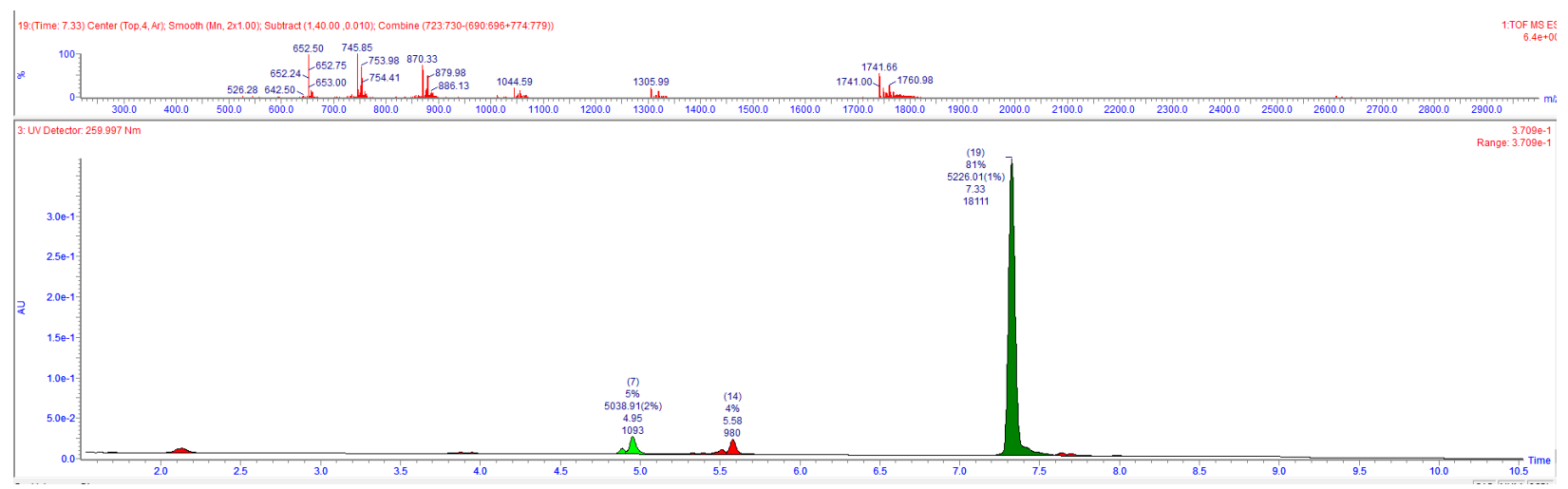




\section{LC Trace and Mass of 40}

Following General Procedure 5A with SI-4 and $250 \mathrm{mM} \mathrm{NaI}$.

Yield: $63 \%$

Exact mass: 5222.03

Triply charged mass [M-3]/3, caluculated: 1739.68; observed 1739.68
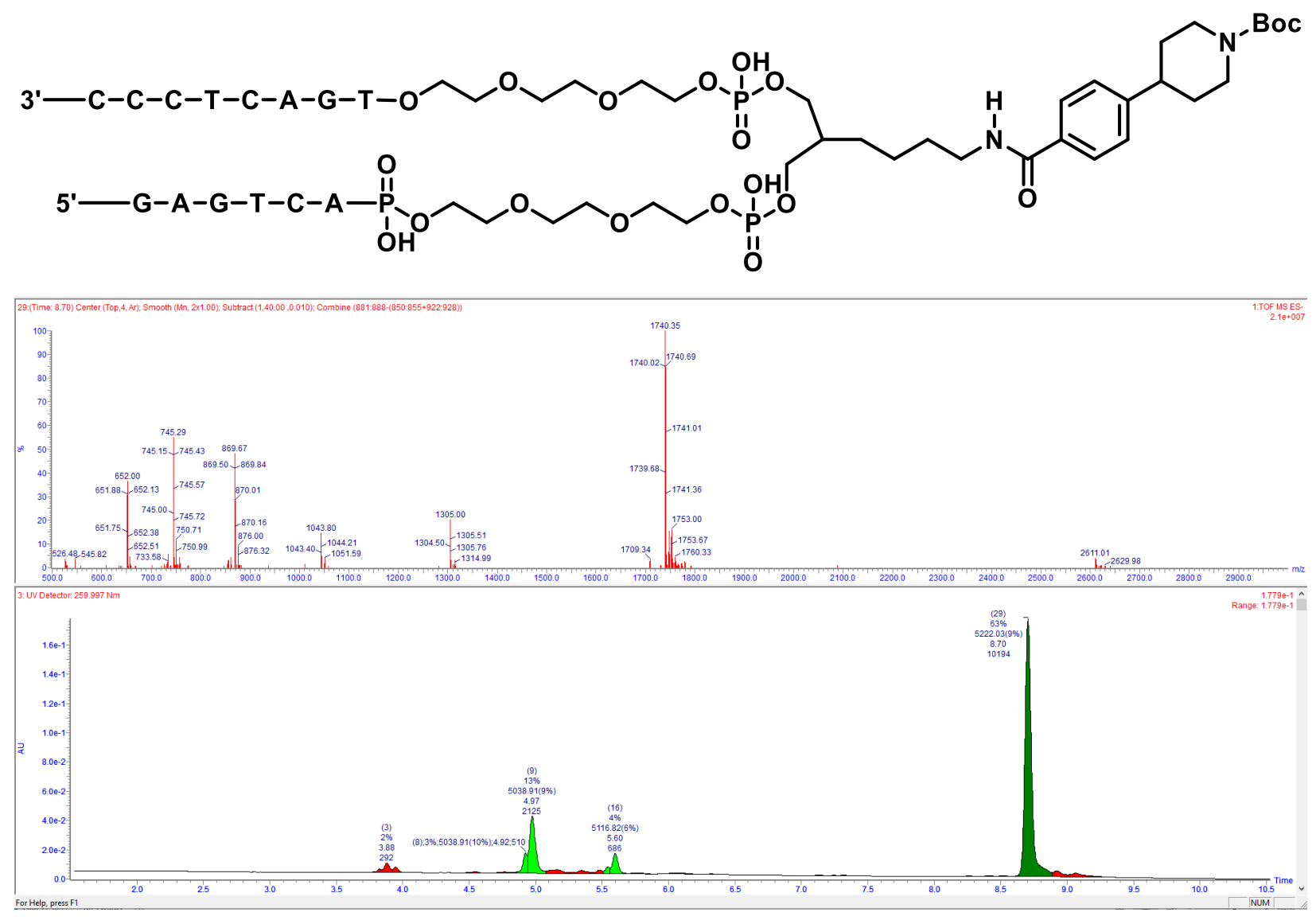


\section{LC Trace and Mass of 40 in tripliacate}

Following General Procedure 5B with SI-4 and $250 \mathrm{mM} \mathrm{NaI}$.

Yields: $70 \%, 64 \%, 52 \%$

Average Yield: $62 \%$

SD: 9.2

Exact mass: 5222.03

Triply charged masses [M-3]/3, caluculated: 1739.68; observed 1741.00, 1741.00, 1741.00
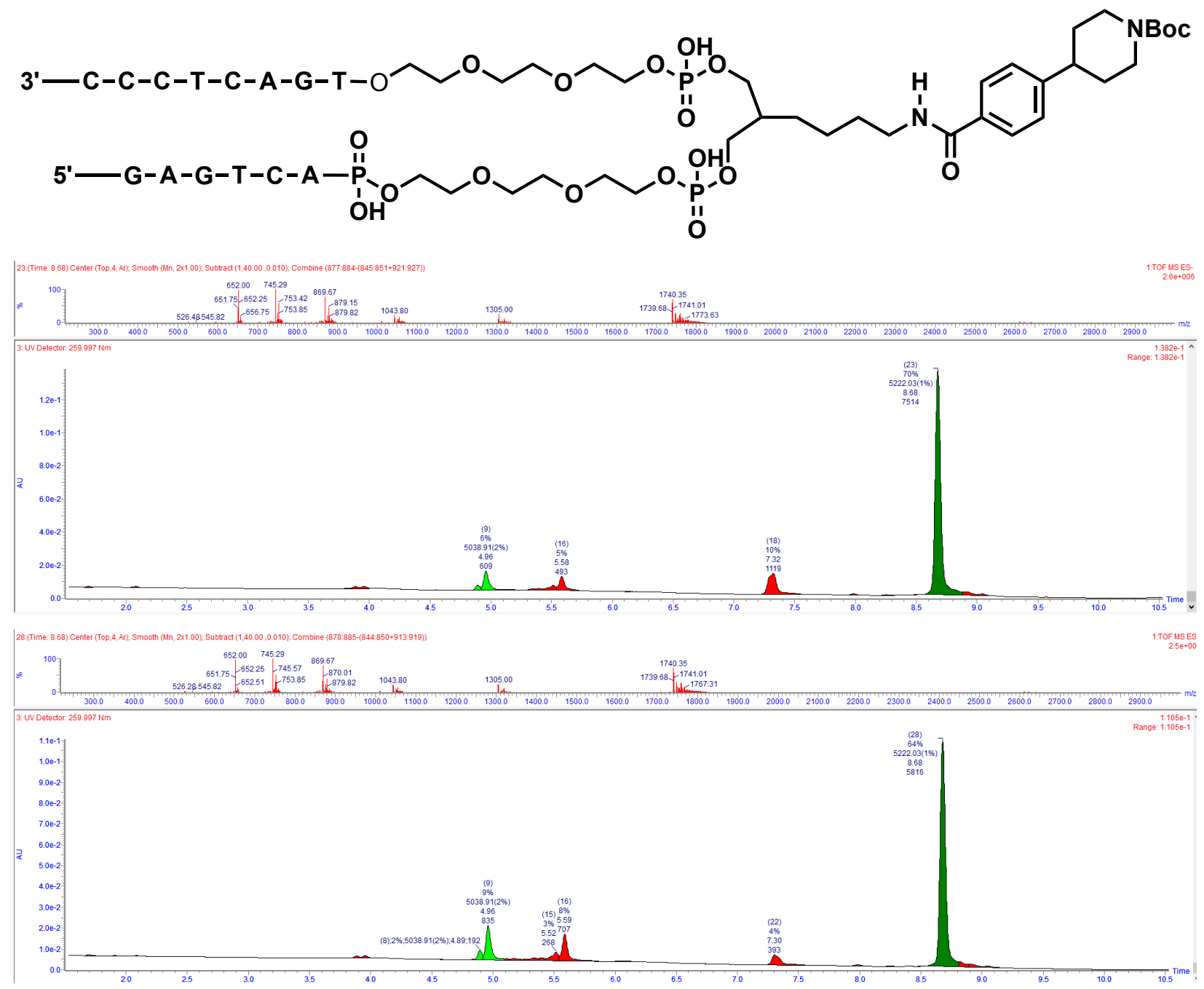


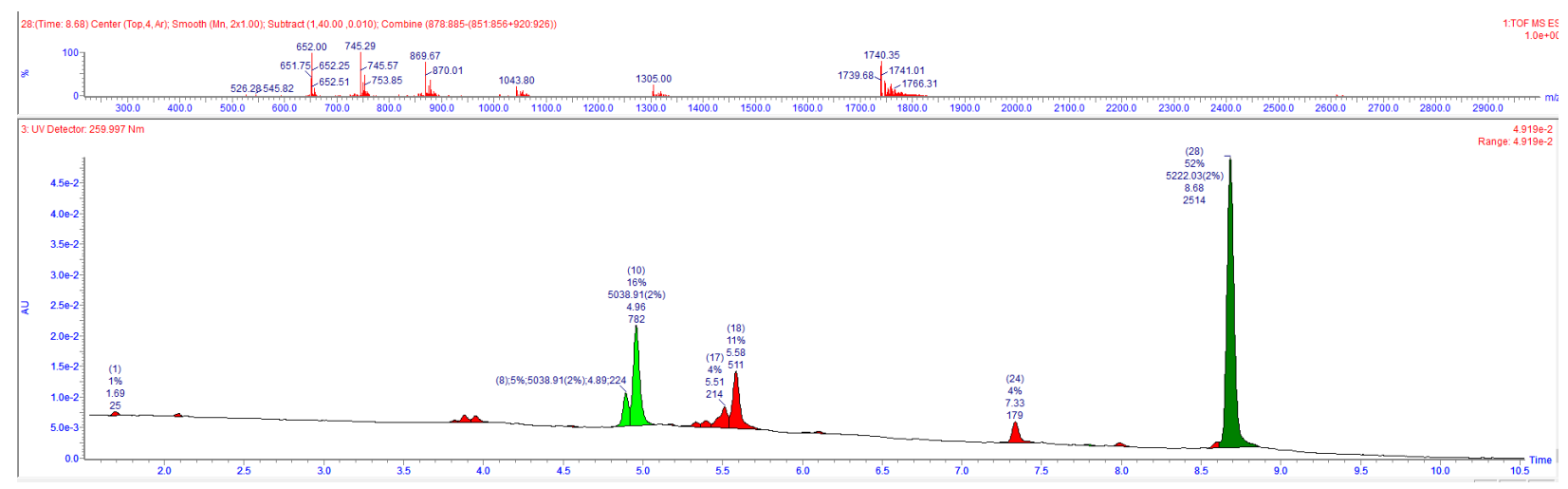




\section{LC Trace and Mass of 41}

Following General Procedure 5A with SI-4 and $250 \mathrm{mM} \mathrm{NaI}$.

Yield: $50 \%$

Exact mass: 5175.99

Triply charged mass [M-3]/3, caluculated: 1757.66; observed 1757.66
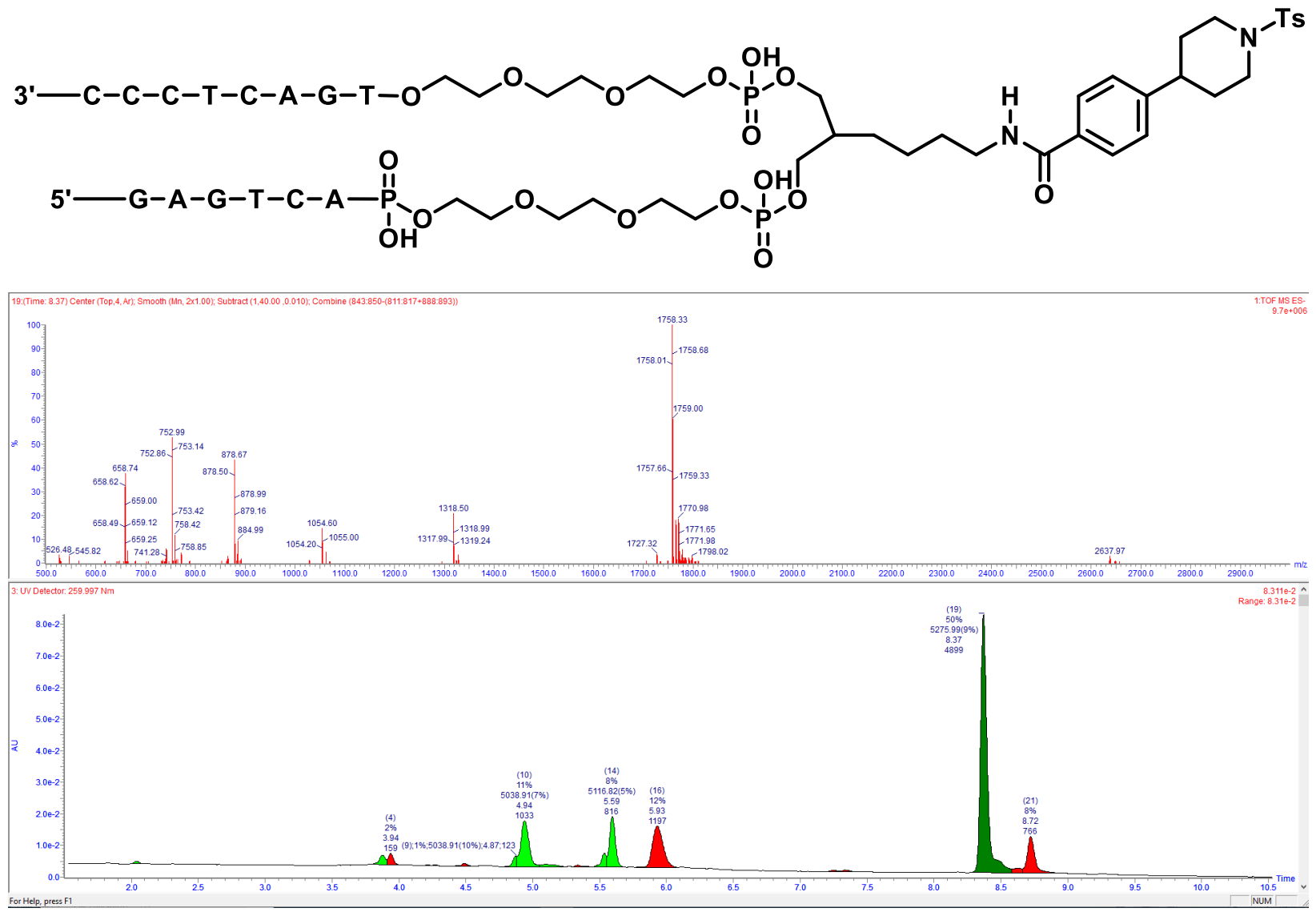


\section{LC Trace and Mass of 42 in tripliacate}

Following General Procedure 5B with SI-4 and $250 \mathrm{mM} \mathrm{NaI}$.

Yields: $68 \%, 63 \%, 71 \%$

Average Yield: $67.33 \%$

SD: 4.0

Exact mass: 5194.00

Triply charged masses [M-3]/3, caluculated: 1730.33; observed 1730.33, 1730.33, 1730.33
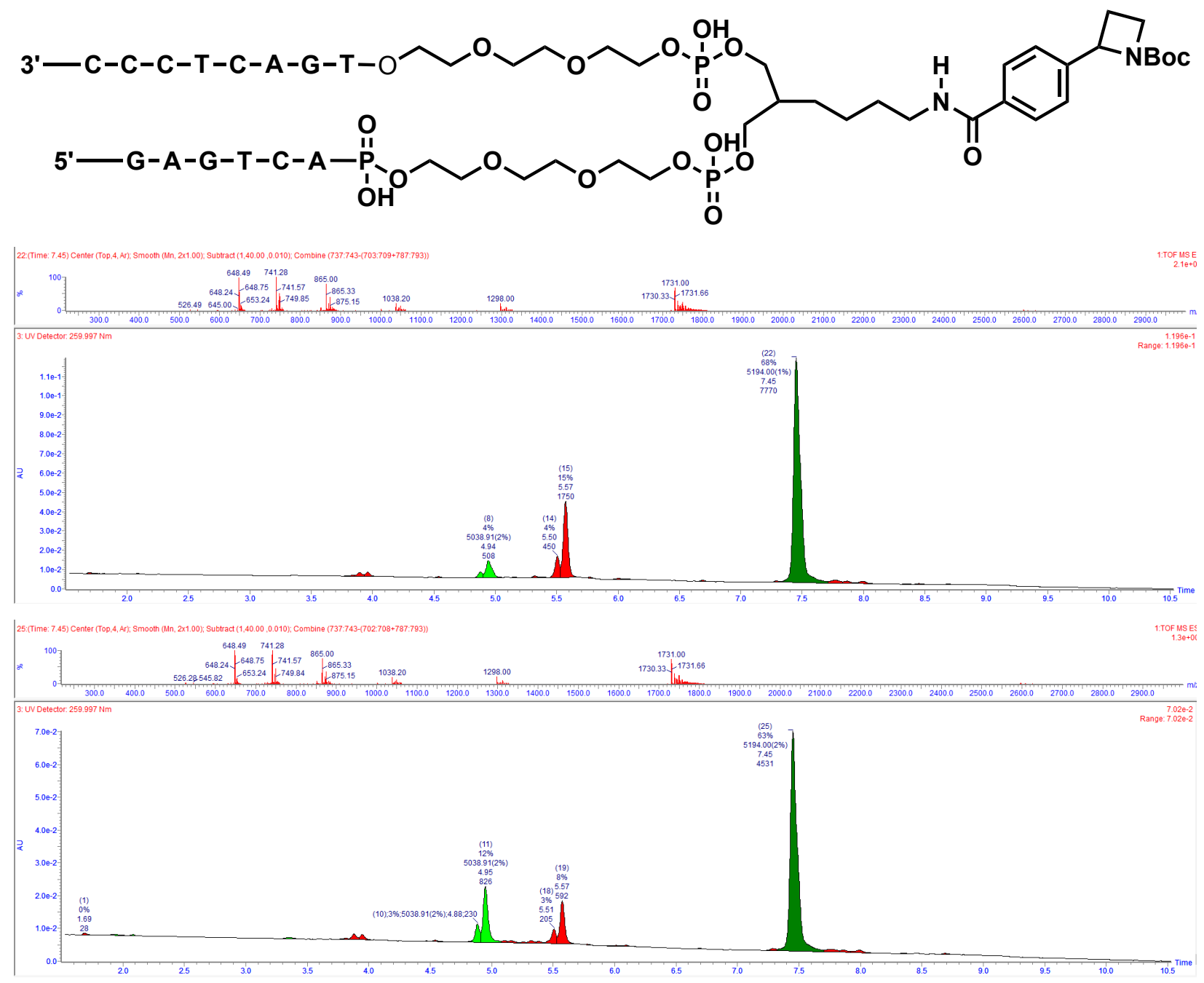


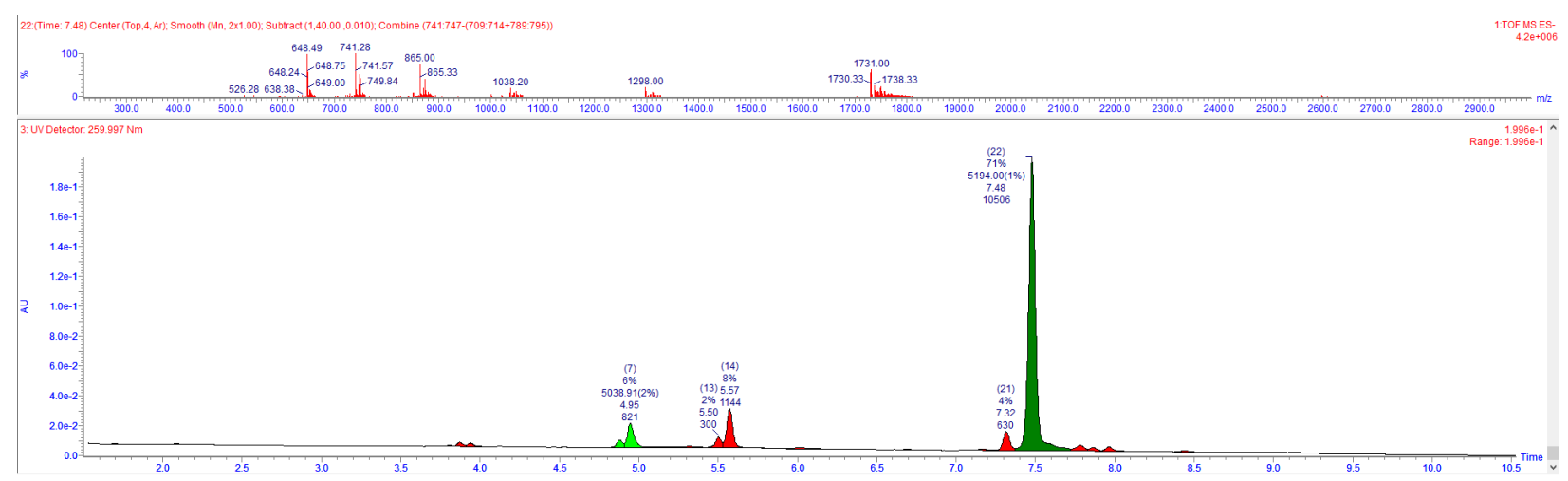




\section{LC Trace and Mass of 43}

Following General Procedure 5A with SI-4 and $250 \mathrm{mM} \mathrm{NaI}$.

Yield: $84 \%$

Exact mass: 5208.02

Triply charged mass [M-3]/3, caluculated: 1735.01; observed 1735.00
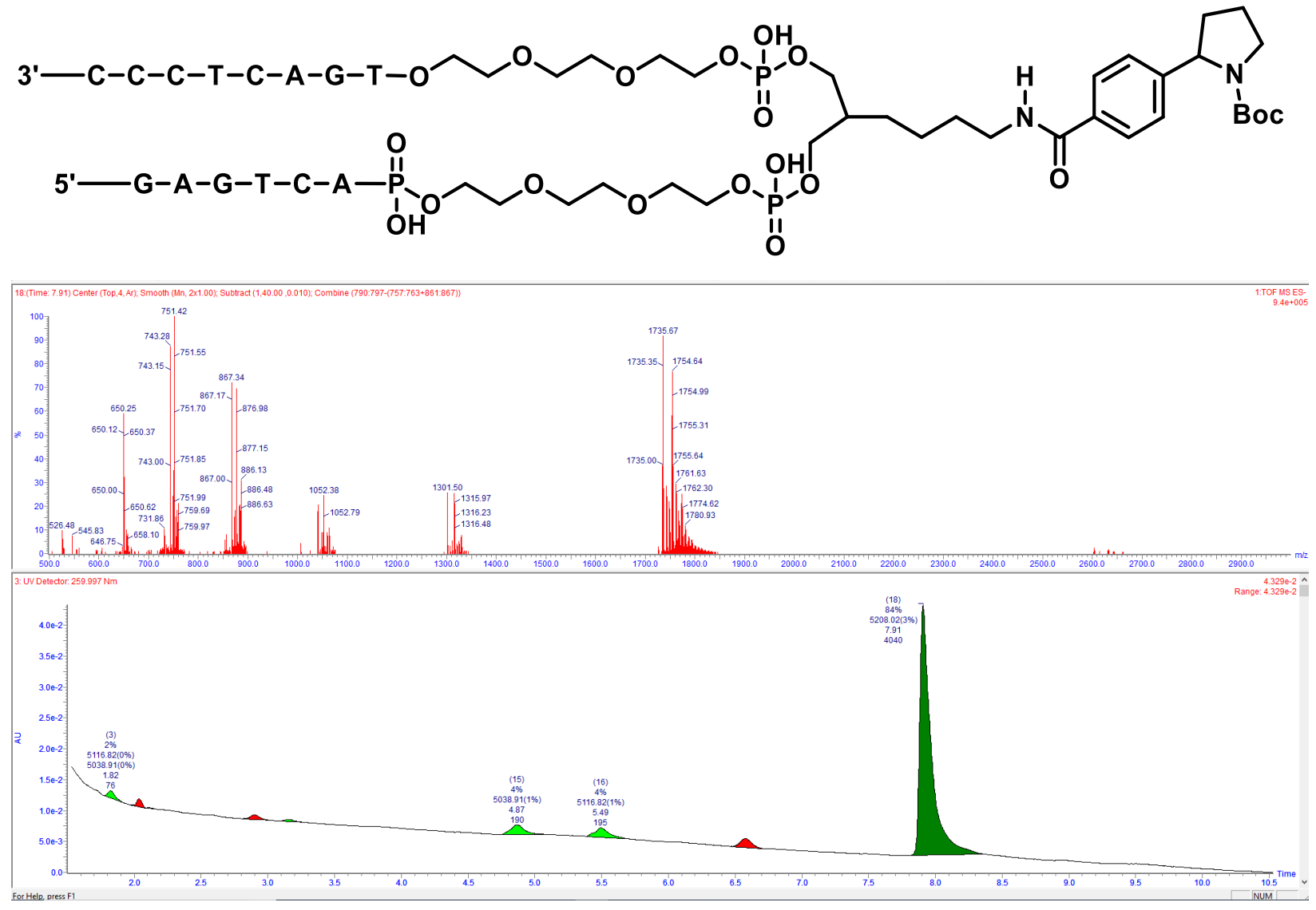


\section{LC Trace and Mass of 44}

Following General Procedure 5A with SI-4 and $250 \mathrm{mM} \mathrm{NaI}$.

Yield: $70 \%$

Exact mass: 5156.99

Triply charged mass [M-3]/3, caluculated: 1718.00; observed 1717.99
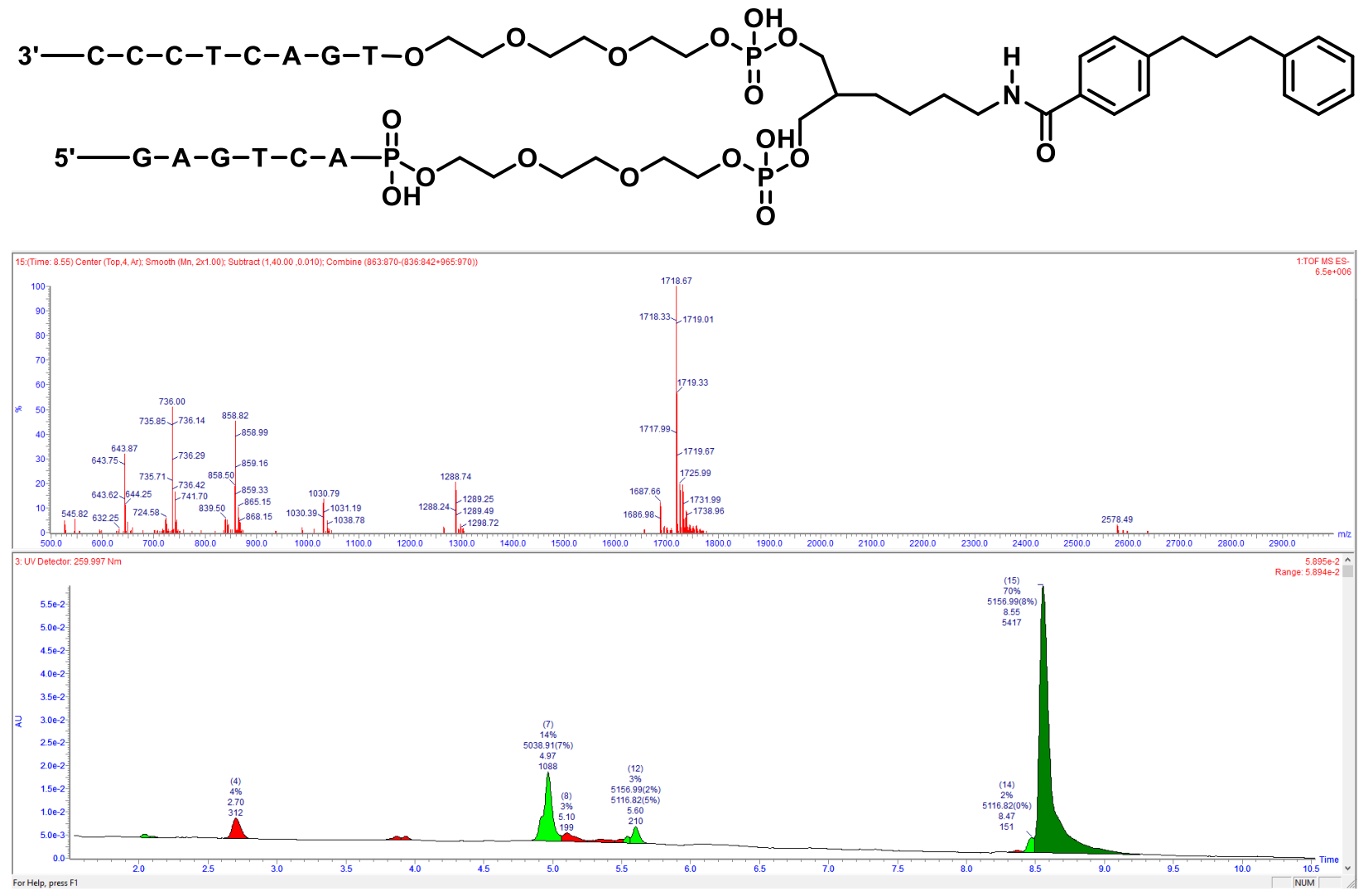


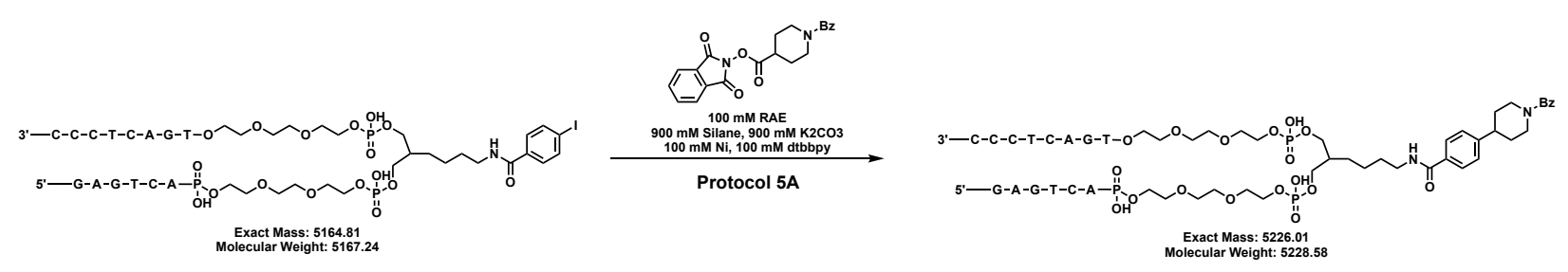

- Phenomenex C18 Silica

- Reversed Phase

$\circ$ Bind: DNA Buffer A (114 mM HFIP and $14 \mathrm{mM} \mathrm{Et}_{3} \mathrm{~N}$ in water)

- Elute: DNA Buffer B (1:1 MeOH:DNA Buffer A)

○ Result: No Product

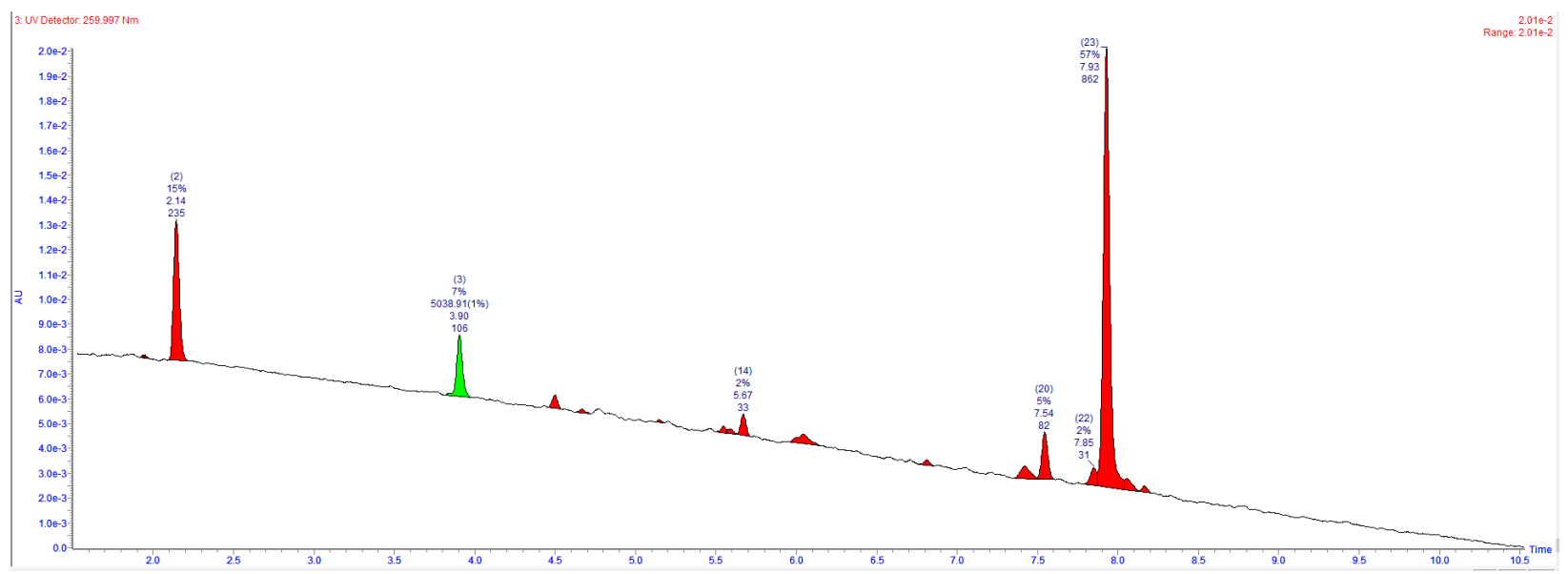

- Phenomenex Phenyl Silica

- Reversed Phase-Phenyl on silica

- Bind: DNA Buffer A (114 mM HFIP and $14 \mathrm{mM} \mathrm{Et}_{3} \mathrm{~N}$ in water)

- Elute: DNA Buffer B (1:1 MeOH:DNA Buffer A)

$\circ$ Result: Trace Product Recovered

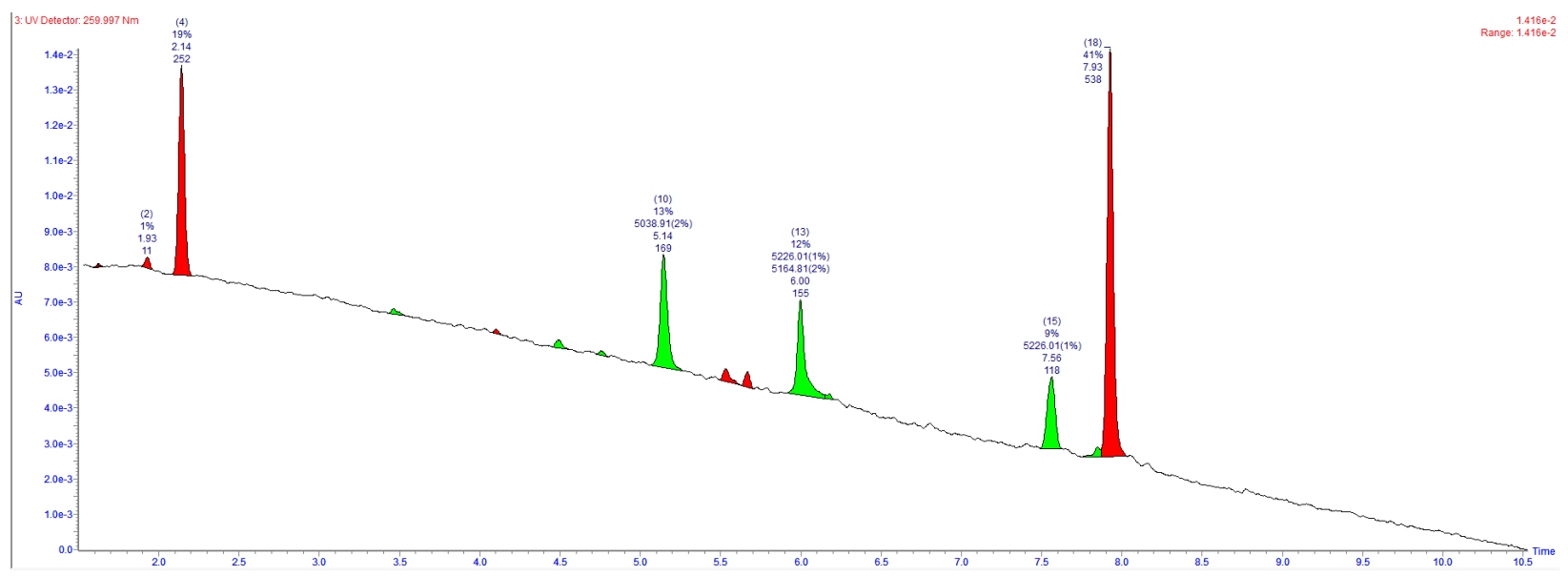


- Phenomenex Styrene Divinyl Benzene

- Reversed Phase-Polystyrene

- Bind: DNA Buffer A (114 mM HFIP and $14 \mathrm{mM} \mathrm{Et}_{3} \mathrm{~N}$ in water)

- Elute: DNA Buffer B (1:1 MeOH:DNA Buffer A)

○ Result: Trace Product Recovered

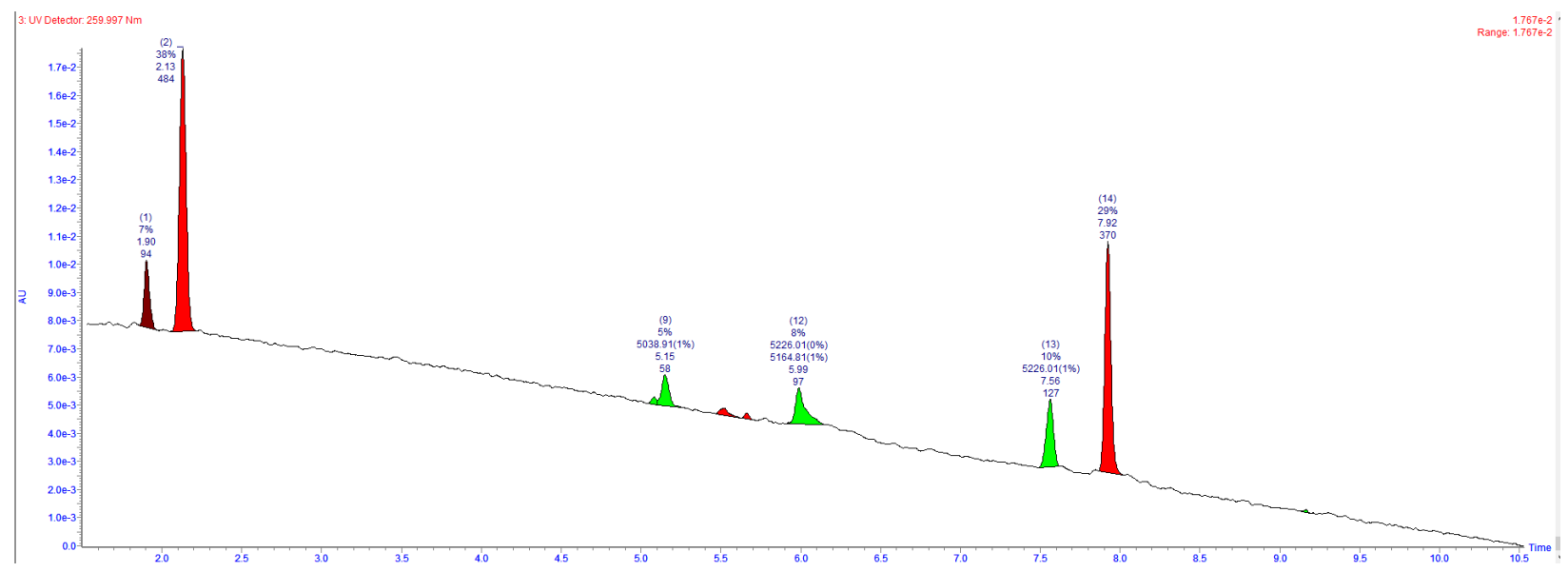

- Phenomenex-Strata-X

- Reversed Phase/Mixed Mode-Pyrollidinone on Polystyrene

- Bind: DNA Buffer A (114 mM HFIP and $14 \mathrm{mM} \mathrm{Et}_{3} \mathrm{~N}$ in water)

- Elute: DNA Buffer B (1:1 MeOH:DNA Buffer A)

○ Result: Trace Product Recovered

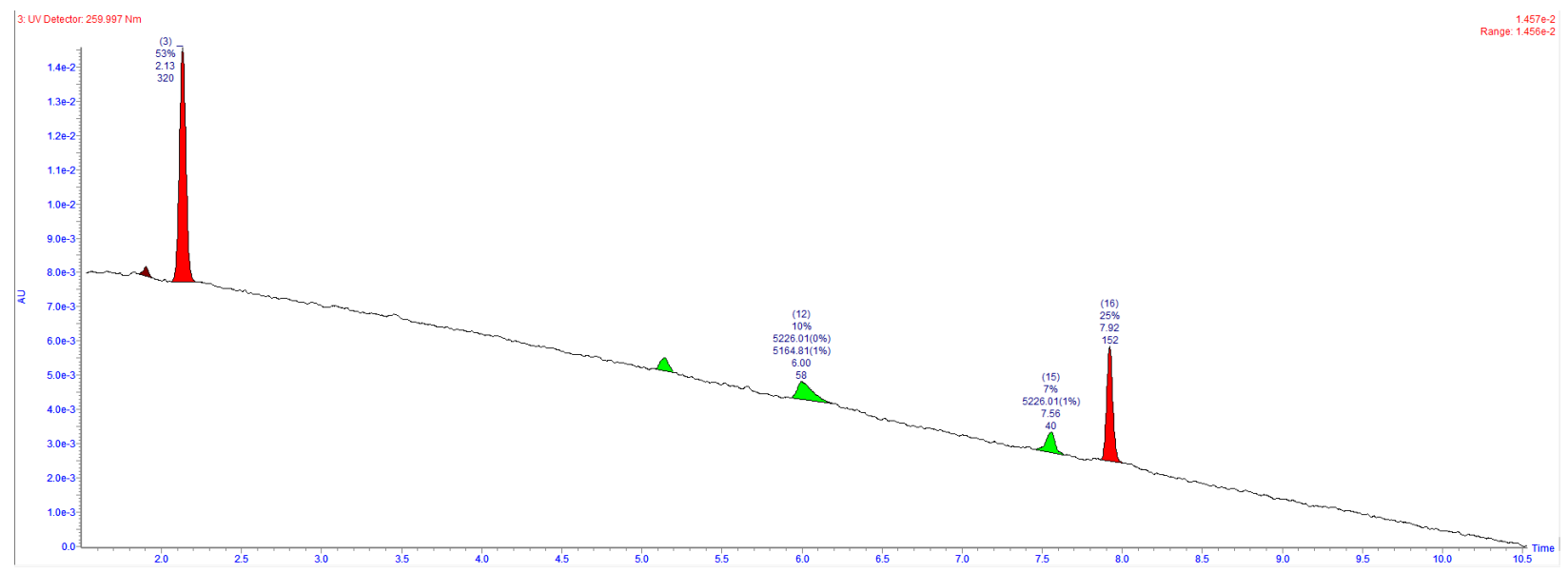


- DEAE Sepharose

- Weak Anion Exchange-Tertiary Amine on Dextran

- Bind: DEAE Binding Buffer (Commercially Available: AcOH Buffer)

- Elute: DEAE Elute Buffer (Commercially Available: Tris Buffer pH 8.5)

- Result: No DNA Recovered

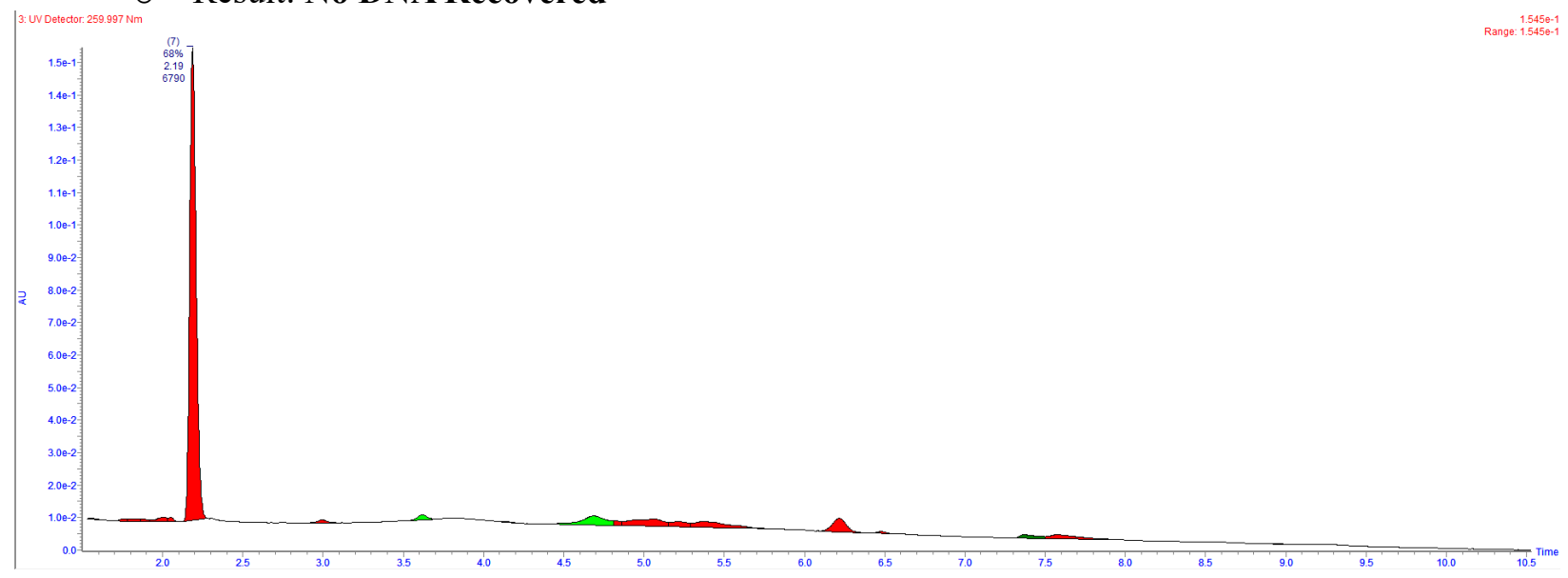

- Phenomenex Strata AW

- Weak Anion Exchange-Primary and Secondary Amine on PolyStyrene

- Bind: DEAE Binding Buffer (Commercially Available: AcOH Buffer)

- Elute: DEAE Elute Buffer (Commercially Available: Tris Buffer pH 8.5)

○ Result: Trace Product Recovered

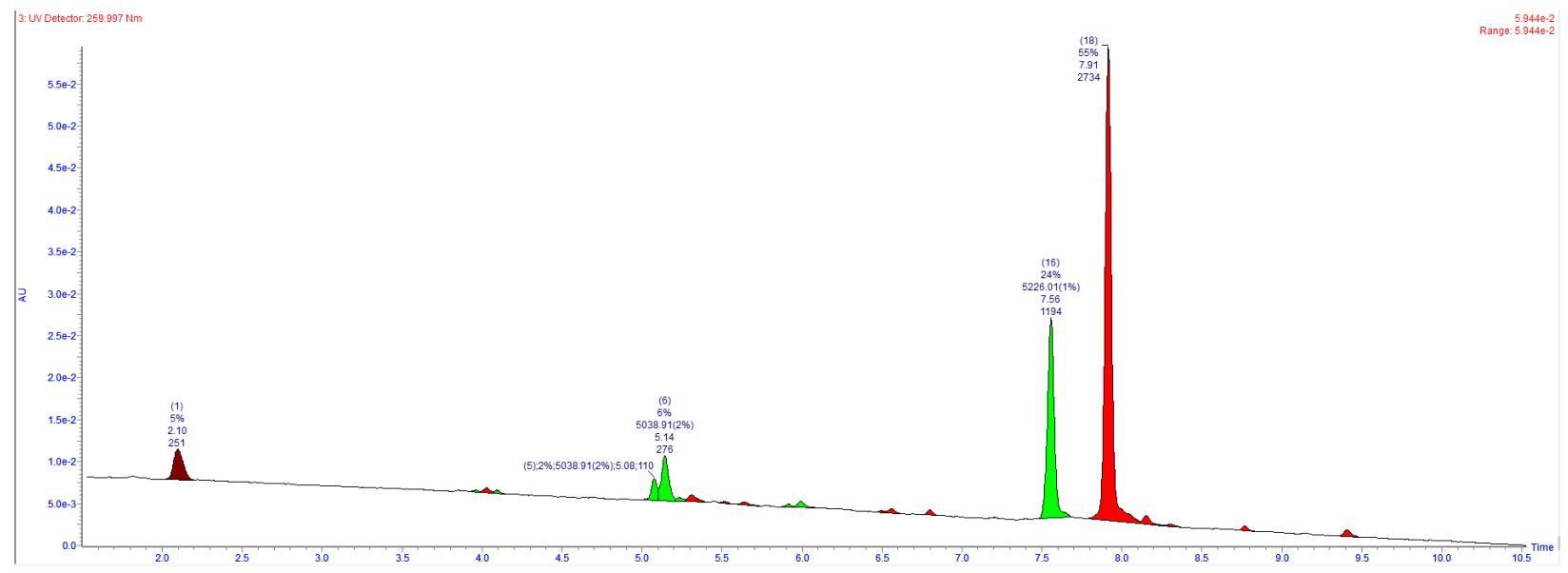


- Waters Oasis MAX

- Strong Anion Exchange-Quaternary Amine on Polystyrene with Pyrrolidinone Functionality

$\circ$ Bind: PBS Binding Buffer (Commercially Available pH 7.4)

- Elute: Elute Buffer $\left(1 \mathrm{M} \mathrm{NaClO}_{4}, 40 \mathrm{mM}\right.$ Tris pH 8.4, 20\% MeOH)

○ Result: $67 \%$ Product

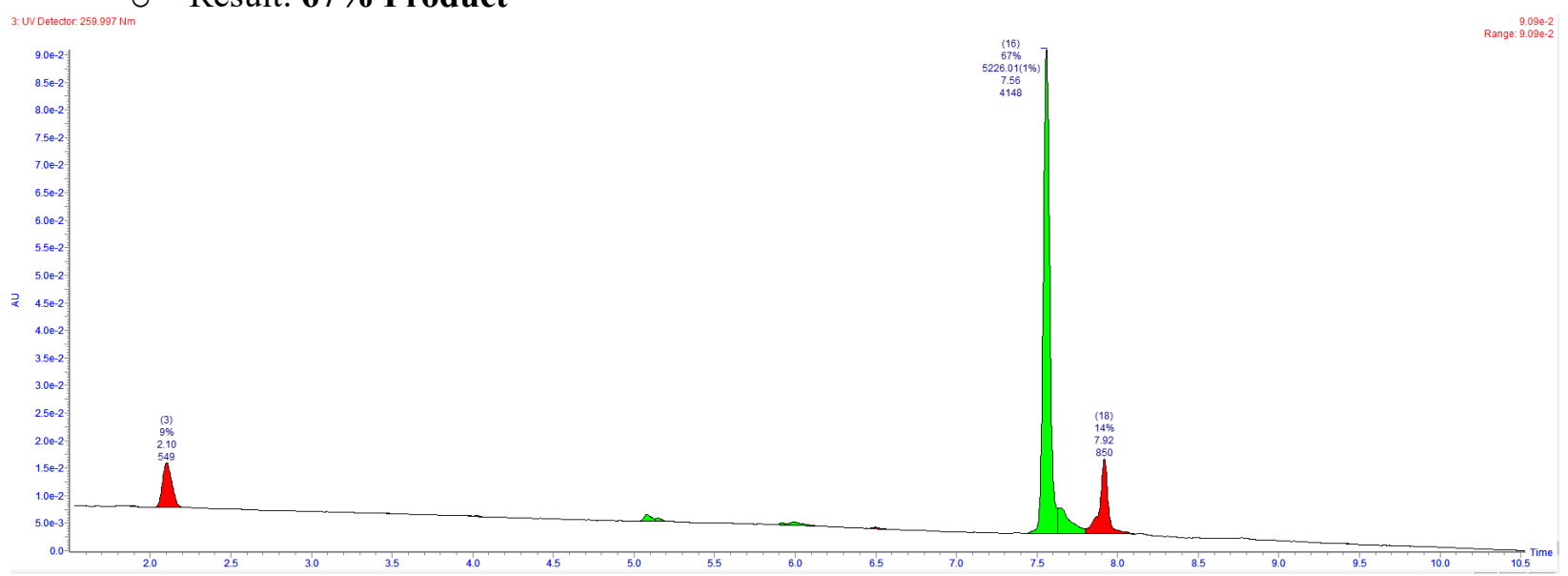

- Phenomenex Strata AXL

- Strong Anion Exchange-Quaternary Amine on Polystyrene (100 um particle, 300 A Pore)

$\circ$ Bind: PBS Binding Buffer (Commercially Available pH 7.4)

- Elute: Elute Buffer $\left(1 \mathrm{M} \mathrm{NaClO}_{4}, 40 \mathrm{mM}\right.$ Tris pH 8.4, 20\% MeOH)

○ Result: $\mathbf{7 1 \%}$ Product

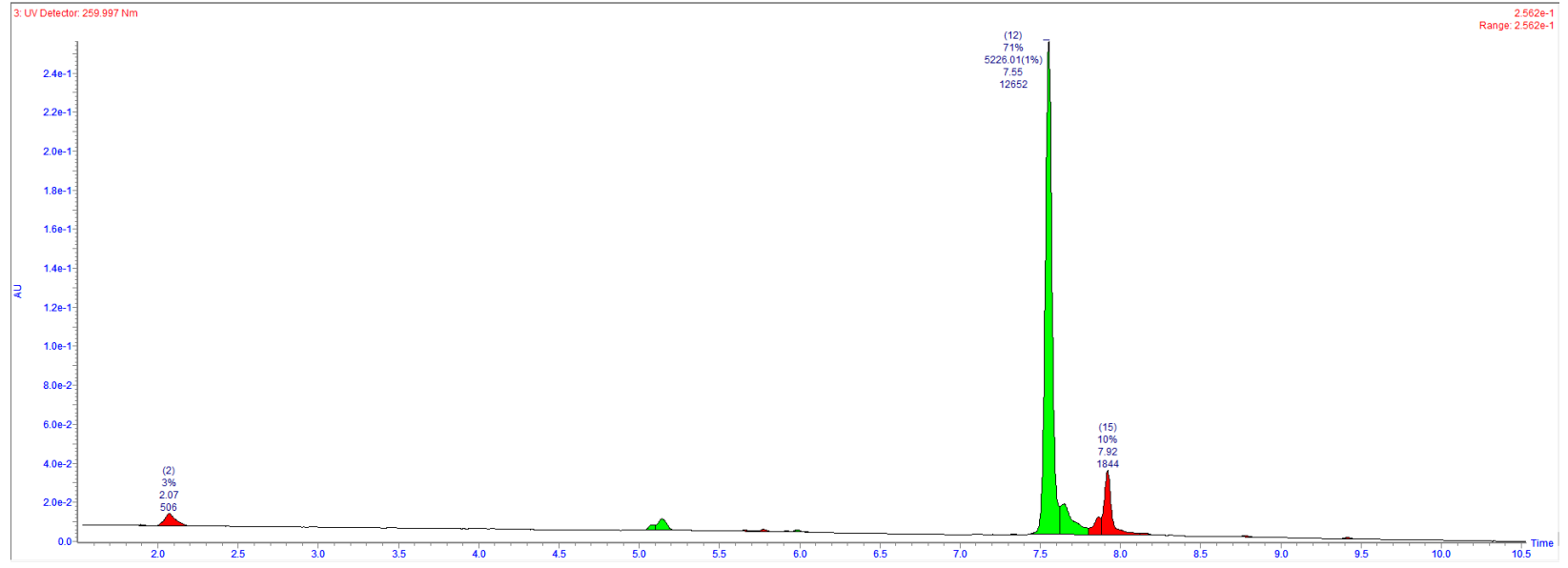




\section{DNA Ligation of sp2-sp3 Coupling Reaction Product}

\section{Primer Ligation}

$3(2 \mathrm{nmol})$ was dissolved in $18 \mu \mathrm{L}$ water. Primer duplex was added $(28 \mu \mathrm{L}$ of a $100 \mu \mathrm{M}$ solution in water, 1.4 equivalents), followed by water $(25 \mu \mathrm{L})$ and $8 \mu \mathrm{L}$ 10X DNA ligation buffer. The solution was heated to $95^{\circ} \mathrm{C}$ for $1 \mathrm{~min}$, then cooled to $16^{\circ} \mathrm{C}$ over $10 \mathrm{~min}$. A solution of T4 DNA ligase $(0.8 \mu \mathrm{L}, 30 \mathrm{U} / \mu \mathrm{L})$ was then added and the ligation incubated at $16^{\circ} \mathrm{C}$ for $16 \mathrm{~h}$.

$\begin{array}{lrl} & \text { Sequence of primer }(\mathrm{MW}=12458 \mathrm{Da}): \\ 5 & \text { AAATCGATGTGGTCAGGAAG } & 3 \\ 3 & \text { GGTTTAGCTACACCAGTCCT }\end{array}$

Sequence of the extended headpiece $(\mathrm{MW}=17606 \mathrm{Da})$ :

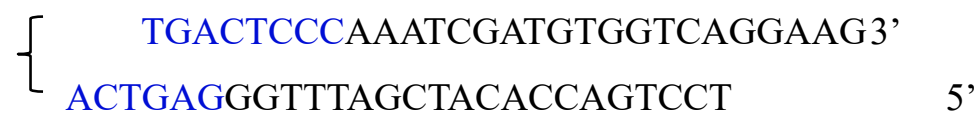

\section{DNA Gel}

Without purification, $1 \mu \mathrm{L}$ of the primer ligation sample was added to $4 \mu \mathrm{L}$ water and $1 \mu \mathrm{L}$ Gel Loading Dye, Purple (6X), no SDS. $1 \mu \mathrm{L}$ of $\mathbf{3}$ ( $1 \mathrm{mM}$ in water) was added to $4 \mu \mathrm{L}$ water and $1 \mu \mathrm{L}$ Gel Loading Dye, Purple (6X), no SDS. $1 \mu \mathrm{L}$ of 3 (1 mM in water) was added to $4 \mu \mathrm{L}$ water and $1 \mu \mathrm{L}$ Gel Loading Dye, Purple (6X), no SDS. $1 \mu \mathrm{L}$ of primer duplex $(100 \mu \mathrm{M})$ was added to $4 \mu \mathrm{L}$ water and $1 \mu \mathrm{L}$ Gel Loading Dye, Purple (6X), no SDS. Then, $5 \mu \mathrm{L}$ of each sample was loaded onto a $5 \%$ agarose gel, which had been freshly cast with $7.5 \mathrm{~g}$ UltraPure Agarose, $150 \mathrm{~mL} 1 \mathrm{X}$ TAE buffer and $15 \mu \mathrm{L}$ 10,000X SYBR ${ }^{\mathrm{TM}}$ Safe DNA Gel Stain. The gel was run in $1 \mathrm{X}$ TAE buffer at $150 \mathrm{~V}$ for 30 minutes. 


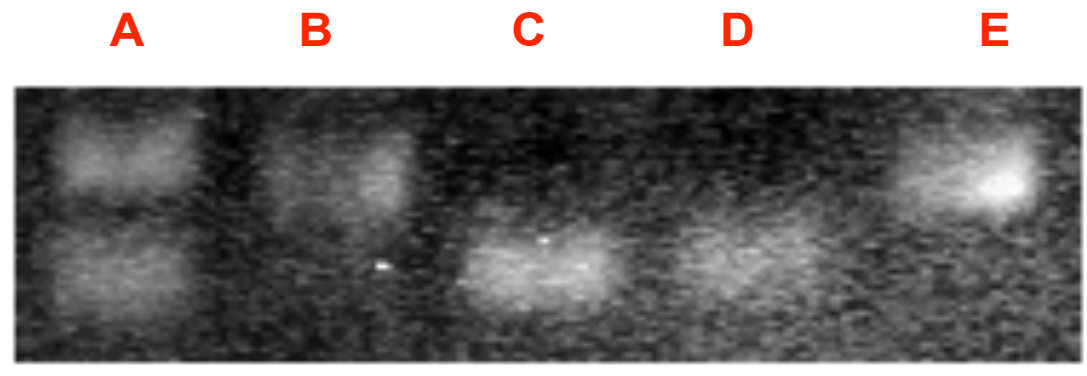
A. Ligation: 1 and Primer
B. Ligation: 3 and Primer
C. 1
D. 3
E. Primer 
Failed Optimization of on-DNA Decarboxylative $\mathbf{s p}^{2}-\mathrm{sp}^{3}$ Coupling Reaction<smiles>O=C(Cc1ccc(I)cc1)NCc1ccncc1</smiles><smiles>O=C(ON1CCC(C(=O)ON2C(=O)c3ccccc3C2=O)CC1)c1ccccc1</smiles>

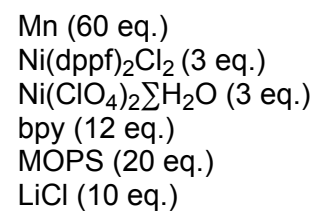

rt, $2 \mathrm{~h}$

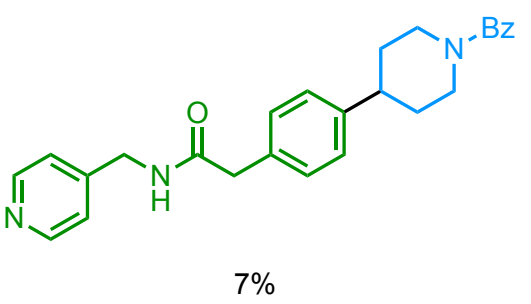




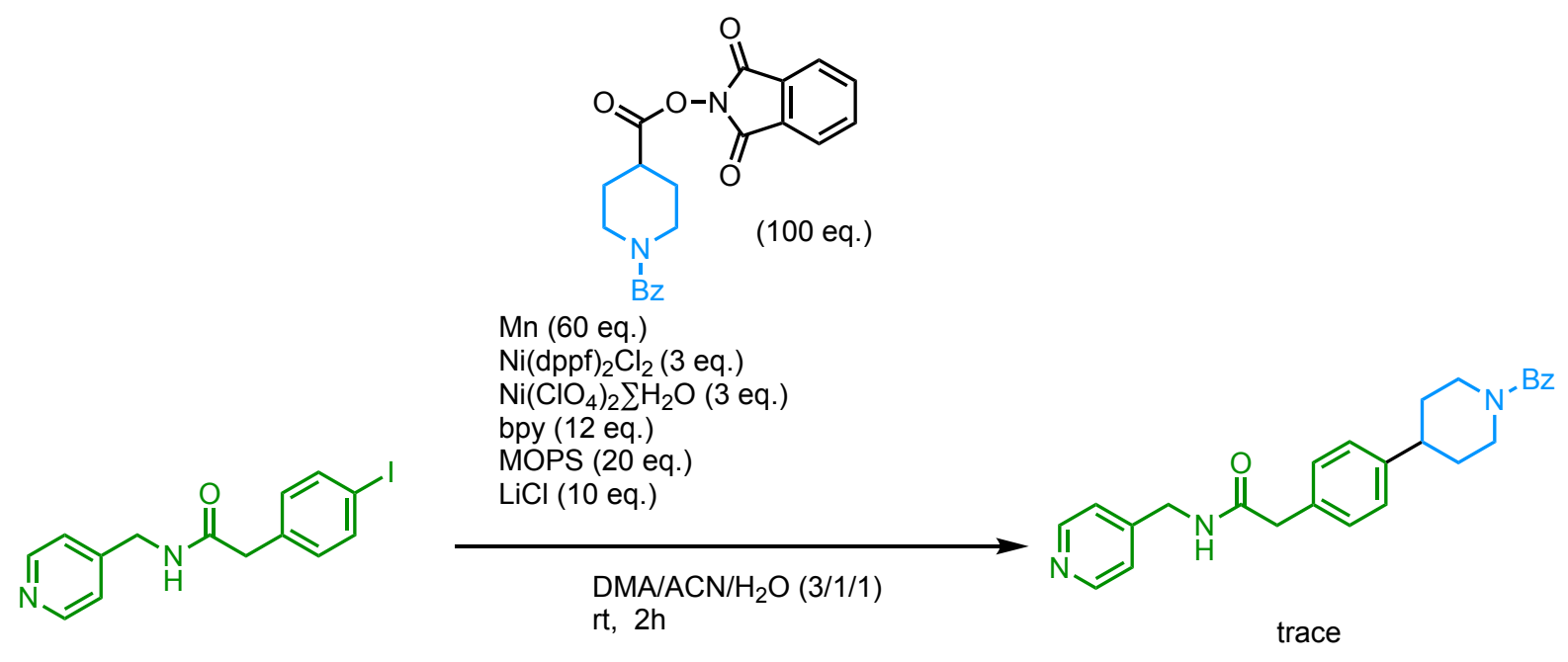




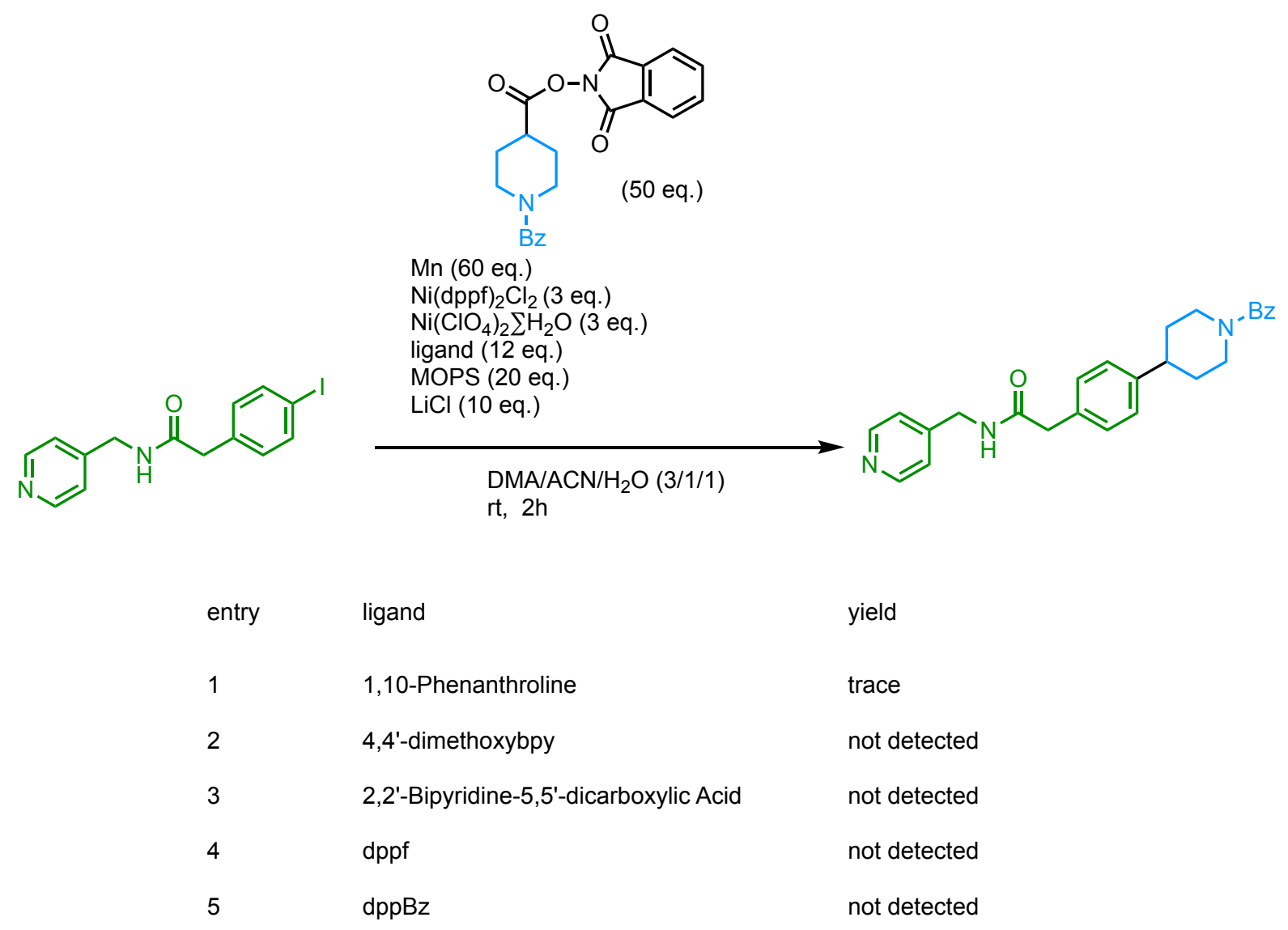




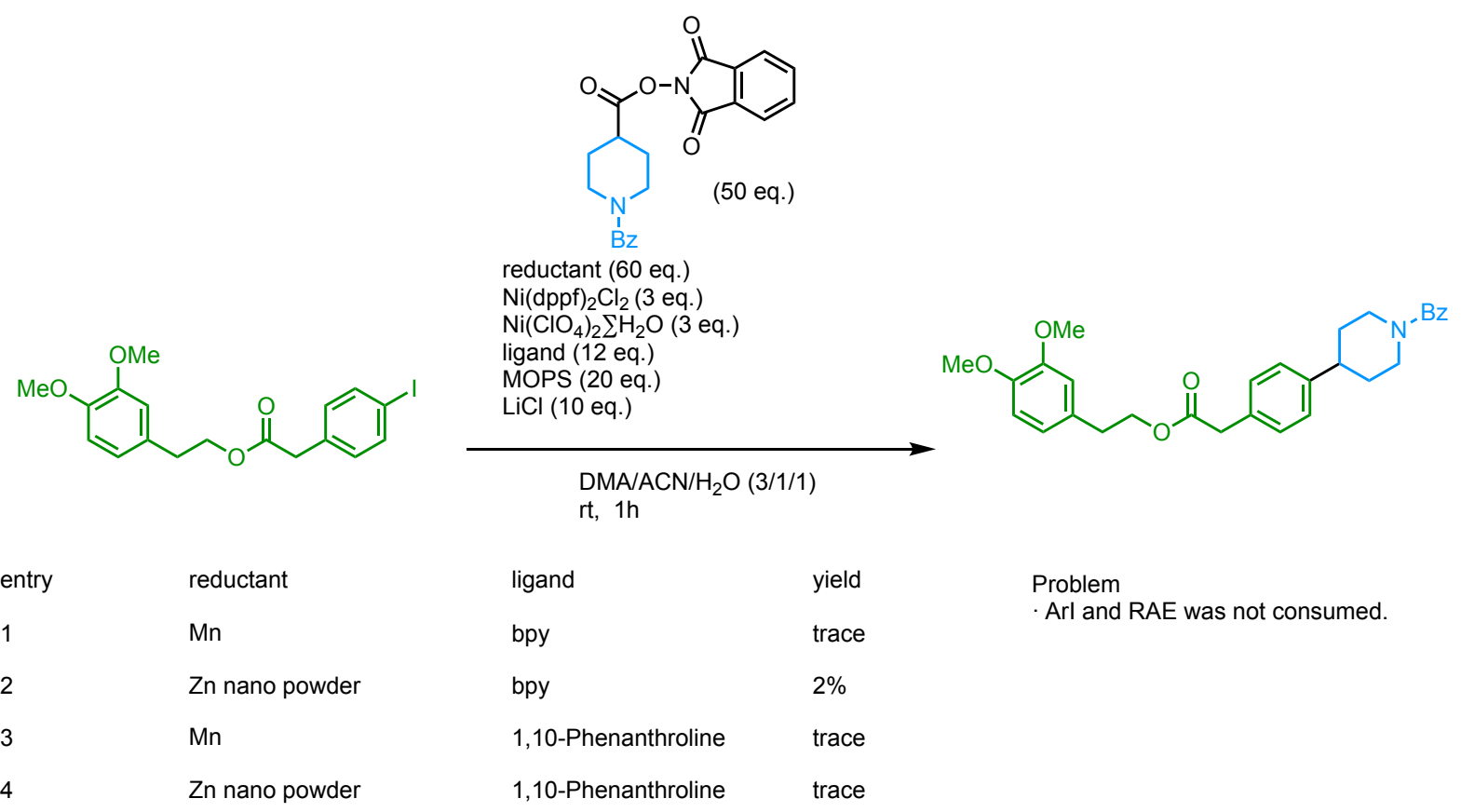



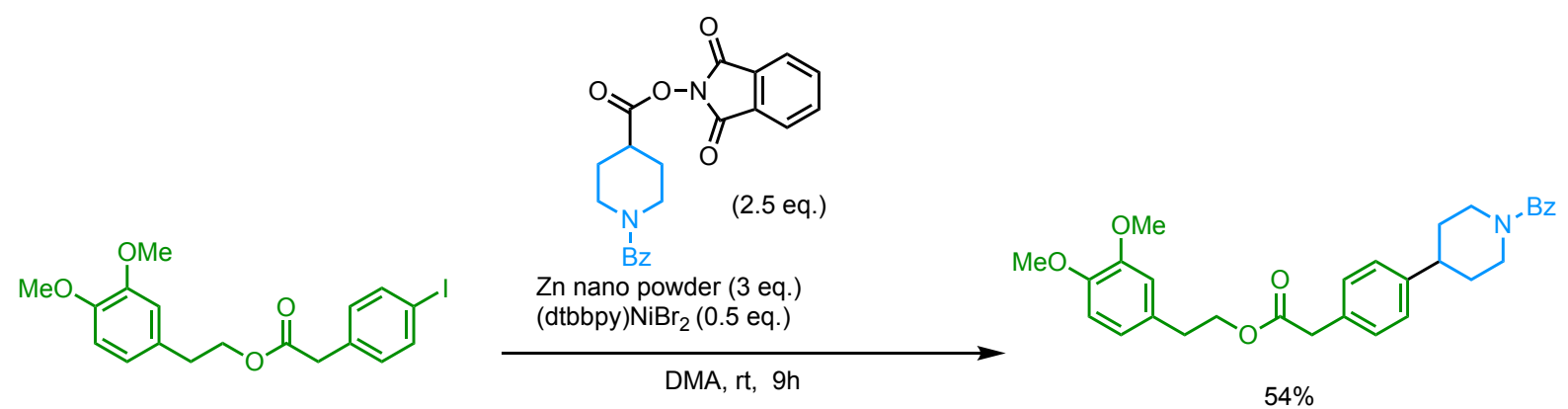


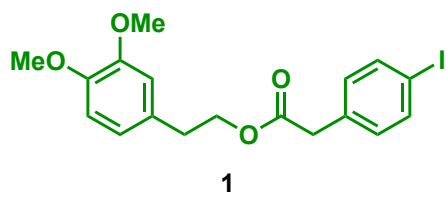

$\begin{array}{ll}\text { entry } & \text { additive } \\ 1 & - \\ 2 & 1 \text { eq. of } \mathrm{H}_{2} \mathrm{O} \\ 3 & 10 \text { eq. of } \mathrm{H}_{2} \mathrm{O} \\ 4 & 100 \text { eq. of } \mathrm{H}_{2} \mathrm{O} \\ 5 & 1000 \text { eq. of } \mathrm{H}_{2} \mathrm{O} \\ 6 & 1 \text { eq. of MOPS } \\ 7 & 5 \text { eq. of MOPS } \\ 8 & 10 \text { eq. of MOPS } \\ 9 & 20 \text { eq. of MOPS } \\ 10 & 0.02 \mathrm{M} \\ 11 & 0.01 \mathrm{M} \\ 12 & 0.002 \mathrm{M} \\ 13 & 0.001 \mathrm{M}\end{array}$

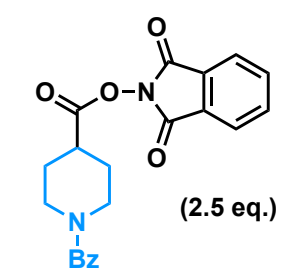

Zn nano powder (3 eq.) (dtbbpy) $\mathrm{NiBr}_{2}$ (0.4 eq.)

DMA (0.1 M), rt, 9-12h
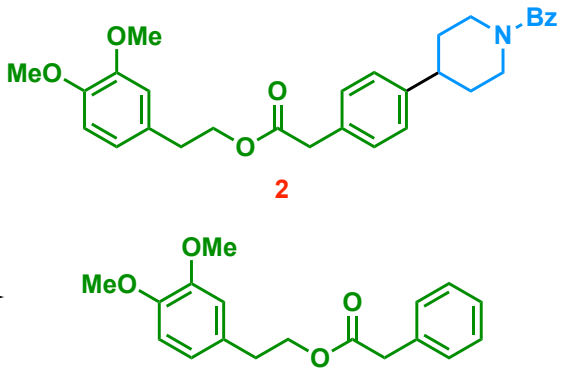

3

yield

$1: 2: 3$ (\%)

$21: 59: 3$

$23: 62: 0$

24: $58: 0$

$37: 4: 42$

$93: 0: 0$

$5: 64: 3$

$15: 62: 4$

$23: 59: 4$

$25: 54: 4$

42: $27: 0$

$55: 17: 0$

$61: 4: 0$

$51: 3: 0$ 
$24: 6$ : -

$0.001 \mathrm{M}, 100$ times of other regents, DMA/ $\mathrm{H}_{2} \mathrm{O}=2 / 1$, premix of $\mathrm{H}_{2} \mathrm{O}$

-: - : -

$0.01 \mathrm{M}, 10$ times of other regents, $\mathrm{DMA} / \mathrm{H}_{2} \mathrm{O}=4 / 1$

$54: 0: 0$

$0.01 \mathrm{M}, 10$ times of other regents, $\mathrm{DMA} / \mathrm{H}_{2} \mathrm{O}=4 / 1,4$ eq. of $\mathrm{Ni}(\mathrm{dppf})_{2} \mathrm{Cl}_{2}$

$87: 0: 0$

$0.01 \mathrm{M}, 10$ times of other regents, $\mathrm{DMA} / \mathrm{H}_{2} \mathrm{O}=4 / 1,4$ eq. of $\mathrm{Ni}(\mathrm{dppf})_{2} \mathrm{Cl}_{2}$

$88: 8: 0$ 10 eq. of $\mathrm{LiCl}$ and MOPS 


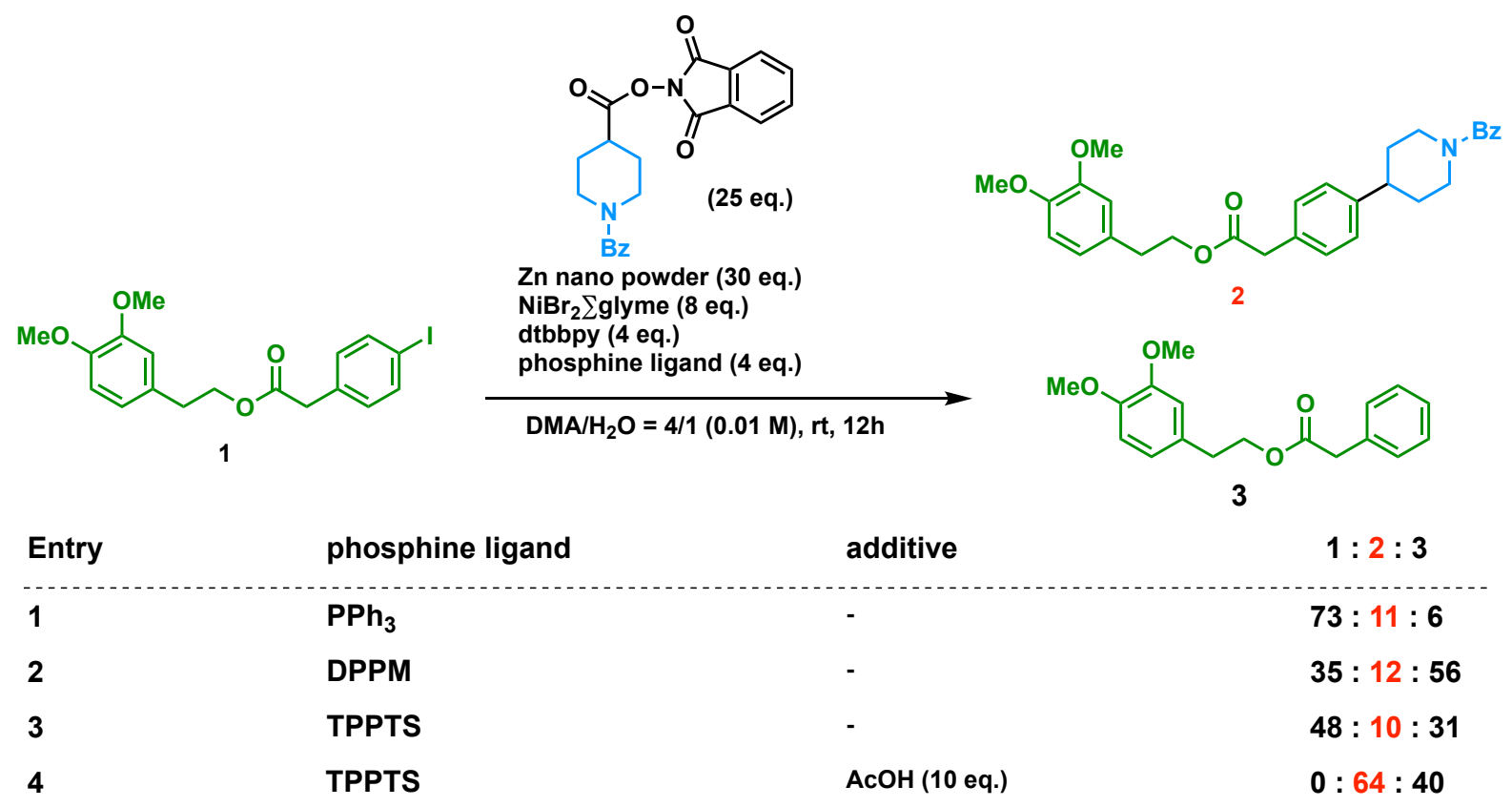




\begin{tabular}{|c|c|}
\hline $3^{\prime}-\mathrm{C}-\mathrm{C}$ & - \\
\hline Entry & Conditions \\
\hline 1 & RAE (2500 eq) , AcOH (1000 eq), RT \\
\hline 2 & $\operatorname{RAE}(2500$ eq), AcOH (10000 eq), RT \\
\hline 3 & $\operatorname{RAE}\left(2500\right.$ eq), $\operatorname{AcOH}\left(1000\right.$ eq), $75^{\circ} \mathrm{C}$ \\
\hline 4 & $\operatorname{RAE}\left(250\right.$ eq) $, \mathrm{AcOH}(1000 \mathrm{eq}), 75^{\circ} \mathrm{C}$ \\
\hline 5 & $\operatorname{RAE}\left(25\right.$ eq) $, \mathrm{AcOH}(1000 \mathrm{eq}), 75^{\circ} \mathrm{C}$ \\
\hline 6 & $\operatorname{RAE}(250 \mathrm{eq}), \mathrm{AcOH}(1000 \mathrm{eq}), \mathrm{RT}$ \\
\hline 7 & RAE (25 eq), AcOH (1000 eq), RT \\
\hline
\end{tabular}



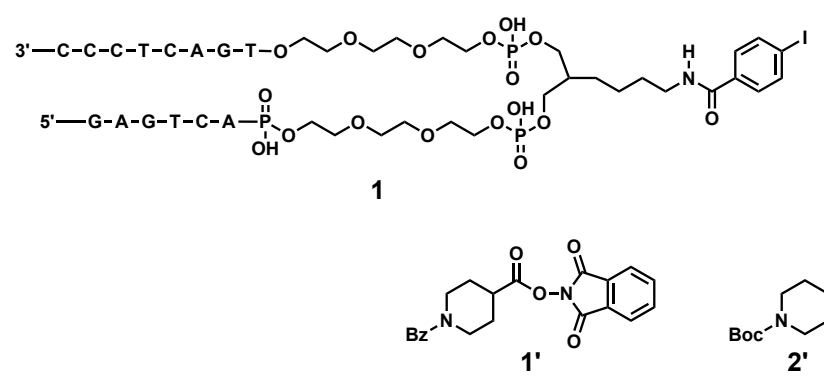

coupling partner (500 eq.)

Zn nano (3000 eq.)

$\mathrm{NiBr}_{2}$ (500 eq.)

tBubipy (250 eq.)

TPPTS (250 eq.)

AcOH (1000 eq.)

DMA/H ${ }_{2} \mathrm{O}=49 / 1$

$2 \mathrm{Hr}$, rt
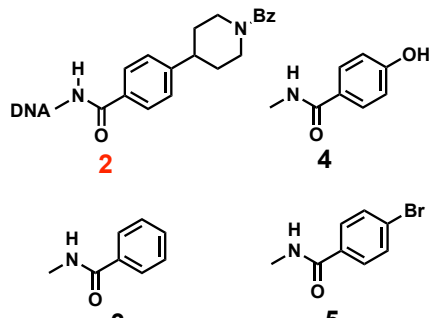

3

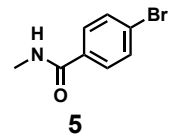

\begin{tabular}{ccc}
\hline Entry & Coupling Partner & $(\%) 1: 0: 3: 4: 5$ \\
\hline 1 & $1^{\prime}$ & $33: 0: 12: 10: 45$ \\
2 & $2^{\prime}$ & $10: 0: 43: 32: 16$ \\
3 & $3^{\prime}$ & $52: 0: 17: 22: 9$ \\
4 & $4^{\prime}$ & $21: 0: 35: 26: 18$ \\
\hline
\end{tabular}




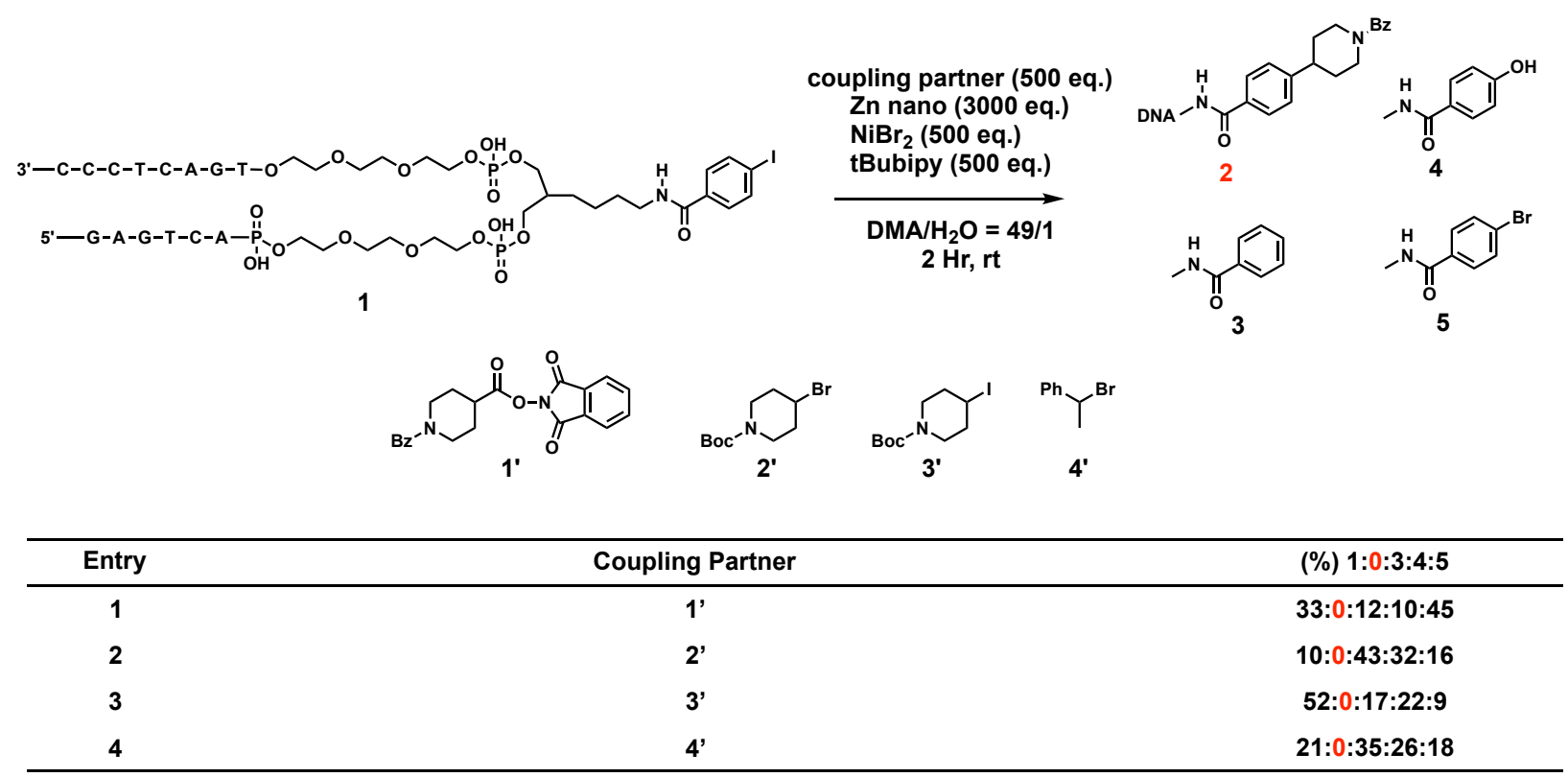




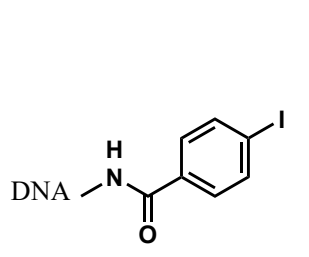

1 acrylate (500 eq.)

Zn nano (3000 eq.)

$\mathrm{NiBr}_{2}$ (500 eq.)

tBubipy (250 eq.)

additive

DMA/ ${ }_{2} \mathrm{O}=4 / 1$

rt, 2 h
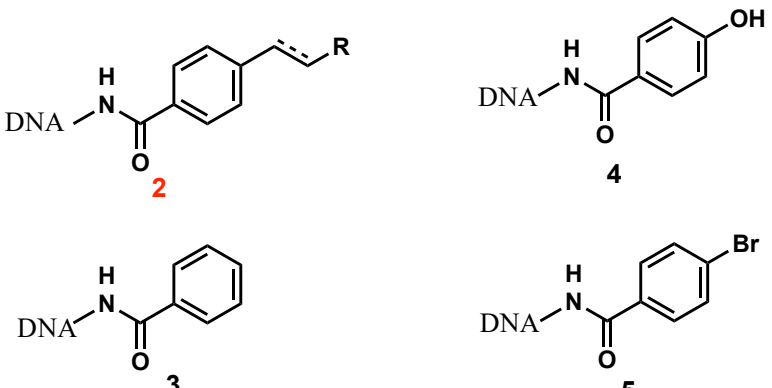

3

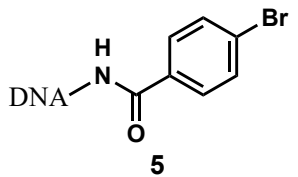

ratio $1: 2: 3: 4: 5$

97:0:3:0:0

28:0:50:9:12

65:0:24:7:4

33:0:48:12:7

26:0:35:23:16

70:0:7:23:0

Not detect DNA Not detect DNA<smiles>C=CC(=O)OCC</smiles>

$\gtrless_{2}^{\mathrm{CN}}$




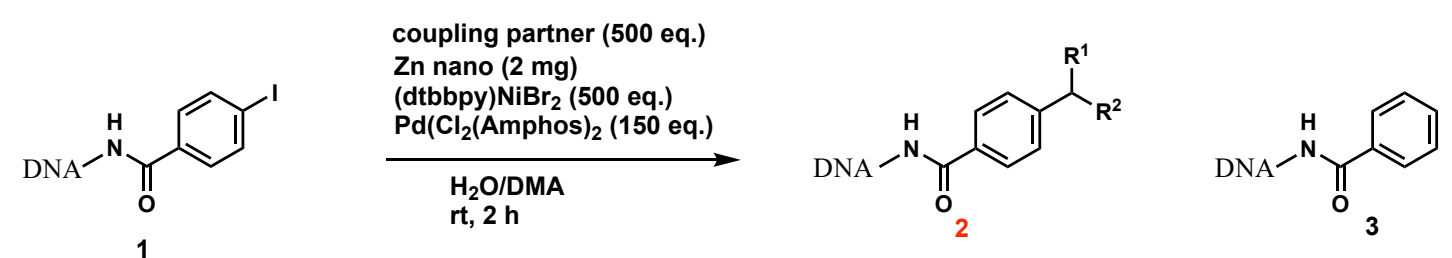

\begin{tabular}{|c|c|c|c|}
\hline Entry & coupling partner & condtion & f $1: 2: 3$ \\
\hline 1 & $1^{\prime}$ & $\mathrm{H}_{2} \mathrm{O} / \mathrm{DMA}=4 / 1$ & $77: 1: 4$ \\
\hline 2 & $1^{\prime}$ & $\mathrm{H}_{2} \mathrm{O} / \mathrm{DMA}=4 / 1, \mathrm{w} / \mathrm{TMEDA}$ (250 eq.) & $78: 1: 3$ \\
\hline 3 & $1^{\prime}$ & $\mathrm{H}_{2} \mathrm{O} / \mathrm{DMA}=3 / 2$ & $79: 1: 3$ \\
\hline 4 & $1^{\prime}$ & $\mathrm{H}_{2} \mathrm{O} / \mathrm{DMA}=2 / 3$ & $31: 1: 43$ \\
\hline 5 & $1^{\prime}$ & $\mathrm{H}_{2} \mathrm{O} / \mathrm{DMA}=1 / 4$ & $38: 2: 36$ \\
\hline 6 & $\mathbf{2}^{\prime}$ & $\mathrm{H}_{2} \mathrm{O} / \mathrm{DMA}=4 / 1$ & No reaction \\
\hline 7 & $3^{\prime}$ & $\mathrm{H}_{2} \mathrm{O} / \mathrm{DMA}=4 / 1$ & No reaction \\
\hline 8 & $1^{\prime}$ & $\mathrm{H}_{2} \mathrm{O} / \mathrm{DMA}=4 / 1, \mathrm{Pd}(\mathrm{OAc})_{2}$ was used insted of $\mathrm{Pd}\left(\mathrm{Cl}_{2}(\mathrm{Amphos})_{2}\right.$ & 79:1:6 \\
\hline 9 & $1^{\prime}$ & $\mathrm{H}_{2} \mathrm{O} / \mathrm{DMA}=4 / 1, \mathrm{Pd}(\mathrm{dppf}) \mathrm{Cl}_{2}$. DCM was used insted of $\mathrm{Pd}\left(\mathrm{Cl}_{2}(\mathrm{Amphos})_{2}\right.$ & $78: 1: 2$ \\
\hline 10 & $1^{\prime}$ & $\mathrm{H}_{2} \mathrm{O} / \mathrm{DMA}=4 / 1, \mathrm{Pd}(\mathrm{dba})_{2}$ was used insted of $\mathrm{Pd}\left(\mathrm{Cl}_{2}(\mathrm{Amphos})_{2}\right.$ & $76: 1: 3$ \\
\hline 11 & $1^{\prime}$ & w/o (dtbbpy) $\mathrm{NiBr}_{2}$ & No reaction \\
\hline 10 & $\mathbf{2}^{\prime}$ & w/o (dtbbpy) $\mathrm{NiBr}_{2}$ & No reaction \\
\hline 11 & $3^{\prime}$ & w/o (dtbbpy) $\mathrm{NiBr}_{2}$ & No reaction \\
\hline 12 & $1^{\prime}$ & $\mathrm{H}_{2} \mathrm{O} / \mathrm{DMA}=4 / 1,70^{\circ} \mathrm{C}$ & 4:0:41 \\
\hline
\end{tabular}<smiles>O=C(ON1C(=O)c2ccccc2C1=O)C1CCN(C(=O)c2ccccc2)CC1</smiles>

1 '<smiles>O=C(c1ccccc1)N1CCC(Br)CC1</smiles><smiles>O=C(c1ccccc1)N1CCC(I)CC1</smiles> 


\section{Electrochemical Amination Optimization}

Resin and DNA were prepared by protocol 1. DMA was dried over oven dried $4 \AA$ Molecular Sieves (MS) for at least 30 minutes while bubbling with argon balloon. DMA $(200 \mu \mathrm{L})$ and four $4 \AA$ MS were added to the resin bed which was left to shake for 30 minutes. Catalyst was weighed into an Eppendorf tube and dissolved with $800 \mu \mathrm{L}$ of DMA. Solid amine was dumped in, or liquid amine was added by micropipette and the solution was vortexed until the amine was completely incorporated. Finally the electrolyte (TBAB, $16 \mathrm{mg}$ ) was added to the solution and dissolved. This reaction solution is added to a $2 \mathrm{~mL}$ IKA Electrasyn vial, charged with four $4 \AA$ MS, and a small stir bar. The resin slurry is then added to the IKA vial. Graphite microelectrodes are secured to the IKA vial top, and the vial top is screwed onto the IKA vial. The port on the top of the vial was fitted with a rubber septum and parafilmed to create a tight seal. The vessel was then degassed as described in protocol 5. The reaction vessel was then fitted on the Electrasyn apparatus and the reaction was run at $4 \mathrm{~mA}$ for various amounts of time. The solution turned black after 10 minutes of current. After the reaction was complete, the resin was transferred from the IKA vial to an Eppendorf tube, washed, eluted, EtOH ppt, and analyzed. 


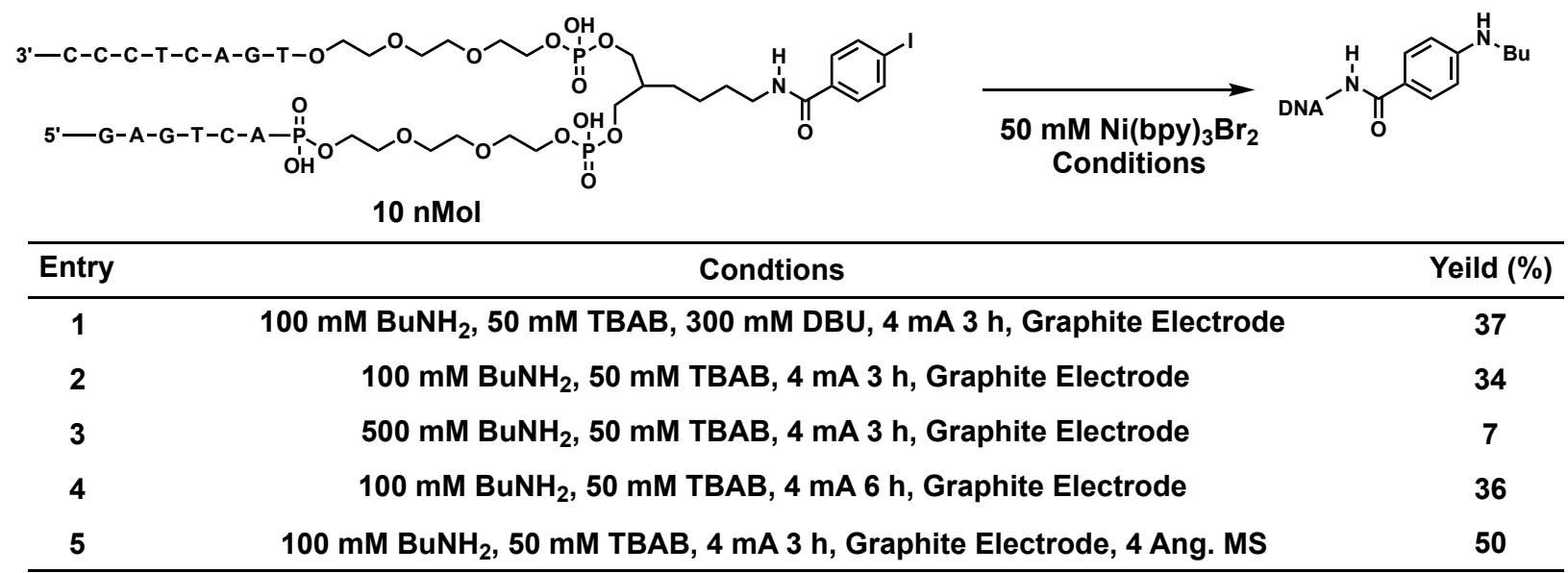

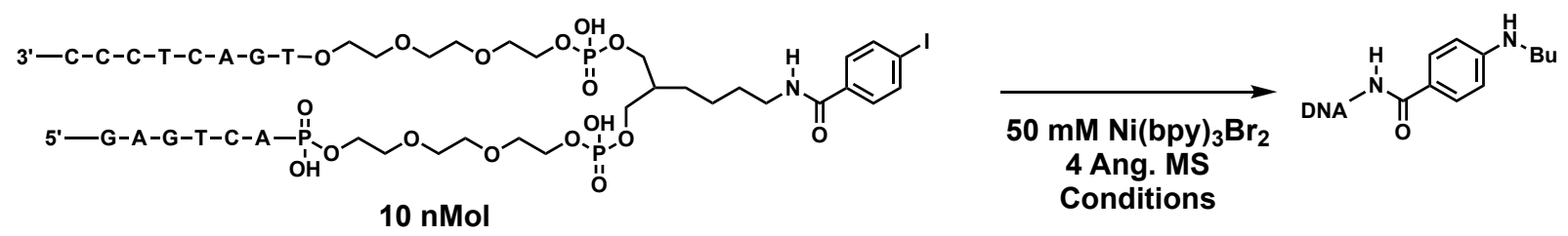

\begin{tabular}{|c|c|c|}
\hline Entry & Condtions & Yeild (\%) \\
\hline 1 & $100 \mathrm{mM} \mathrm{BuNH}_{2}, 50 \mathrm{mM}$ TBAl, $4 \mathrm{~mA} 3 \mathrm{~h}$, Graphite Electrode & 7 \\
\hline 2 & $100 \mathrm{mM} \mathrm{BuNH}_{2}, 50 \mathrm{mM} \mathrm{Bu}_{4} \mathrm{NBF}_{4}, 4 \mathrm{~mA} 3 \mathrm{~h}$, Graphite Electrode & 46 \\
\hline 3 & $100 \mathrm{mM} \mathrm{BuNH}{ }_{2}, 50 \mathrm{mM}$ TBAB, $50 \mathrm{mM} \mathrm{NiBr} 2$ Diglyme, $4 \mathrm{~mA} 3 \mathrm{~h}$, Graphite Electrode & 33 \\
\hline 4 & $100 \mathrm{mM} \mathrm{BuNH}{ }_{2}, 50 \mathrm{mM}$ TBAB, $4 \mathrm{~mA} 3 \mathrm{~h}$, Graphite Electrode, $25 \mathrm{mM} \mathrm{Ni(bpy)}{ }_{3} \mathrm{Br}_{2}$ & 41 \\
\hline 5 & $100 \mathrm{mM} \mathrm{BuNH}$, $50 \mathrm{mM}$ TBAB, $4 \mathrm{~mA} 3 \mathrm{~h}$, Graphite Electrode, $100 \mathrm{mM} \mathrm{Ni(bpy)}{ }_{3} \mathrm{Br}_{2}$ & 74 \\
\hline 6 & $100 \mathrm{mM} \mathrm{BuNH}$, 50 mM TBAB, 4 mA 3 h, Glassy Carbon Electrode & 42 \\
\hline 7 & $100 \mathrm{mM} \mathrm{BuNH}$, $50 \mathrm{mM}$ TBAB, 4 mA 3 h, RVC Electrode & 42 \\
\hline 8 & $\mathrm{X}=\mathrm{Br}, 100 \mathrm{mM} \mathrm{BuNH}{ }_{2}, 50 \mathrm{mM}$ TBAB, $4 \mathrm{~mA} 3 \mathrm{~h}$, Graphite Electrode & 38 \\
\hline
\end{tabular}




\section{Protocol 6: Electrochemical Amination}

1. Prepare resin and DNA as described in Protocol 1.

2. Prepare dry degassed DMA by allowing DMA to sit over oven dried $4 \AA$ MS while purging with nitrogen for at least 20 minutes.

3. Add four $4 \AA$ MS and $200 \mathrm{uL}$ dry DMA to the resin bed and agitate this mixture for at least 15 minutes.

4. Weight $\mathrm{Ni}(\mathrm{bpy})_{3} \mathrm{Br}_{2}(68 \mathrm{mg}, 100 \mathrm{mM}$ in $1 \mathrm{~mL})$ into $2 \mathrm{~mL}$ Eppindorf tube. Vortex and sonicate until the Ni catalyst is mostly dissolved.

5. Add amine (100 mM, in $1 \mathrm{~mL}$ ) to the nickel solution by pipette (liquid amines), or weigh solid amine into an Eppindorf tube and transfer the Ni solution to that tube. Vortex until amine is dissolved.

6. Weigh TBAB (16 mg, $50 \mathrm{mM})$ into a $2 \mathrm{~mL}$ Eppendorf tube and transfer solution of nickel and amine to that tube to dissolve the electrolyte.

7. Once dissolved transfer this reaction solution to a $2 \mathrm{~mL}$ IKA Electrasyn vial that was charged with a small stirbar and four $4 \AA$ MS.

8. Add the resin slurry to the IKA vial by transferring via $1000 \mu \mathrm{L}$ pipette. Total solution volume will be $1 \mathrm{~mL}$

9. Place on a stir plate. Attach the graphite microelectrodes to the IKA vial top. Screw the top onto the IKA vial and perform the degassing procedure while stirring.

10. Degassing procedure:

a. Pierce septa on top of IKA vial cap with needle connected to high vaccuum.

b. Evacuate atmosphere under high vac for 30 seconds.

c. Backfill with argon from balloon.

d. Repeat 3-5 times. Remove balloon and vacuum line and parafilm the septum and reaction vessel leaving the reaction tubes inside the larger test tube.

e. Degassing procedure adapted from: Wang, J.; Lundberg, H.; Asai, S.; Martín-Acosta, P.; Chen, J. S.; Brown, S.; Farrell, W.; Dushin, R. G.; O’Donnell, C. J.; Ratnayake, A. S.; et al. Kinetically Guided Radical-Based Synthesis of C(Sp3)-C(Sp3) Linkages on DNA. PNAS 2018, 201806900.

11. Attach the reaction vial to the IKA Electrasyn, with balloon still attached (providing positive Ar pressure) and parafilm the top to create a tight seal.

12. Run the reaction at $4 \mathrm{~mA}$ for 3-6 hours while stirring at $650-700 \mathrm{rpm}$.

13. Upon completion of electrochemical treatment, transfer resin slurry to $2 \mathrm{~mL}$ Eppindorf tube.

14. Wash, elute, $\mathrm{EtOH}$ ppt, and analyze like before. 


\section{Graphical Supporting Information: On-DNA Electrochemical Amination}

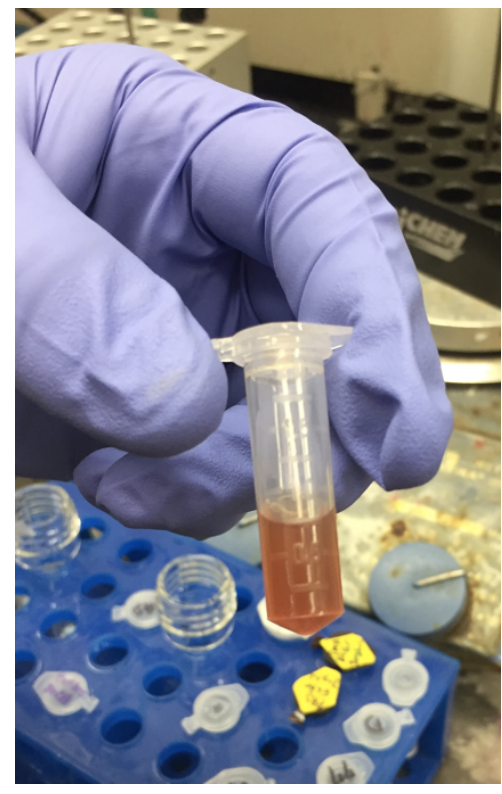

Solution $(800 \mu \mathrm{L})$ of $\mathrm{Ni}(\text { bpy })_{3} \mathrm{Br}_{2}(100 \mathrm{mM})$ and Butylamine (100 mM)

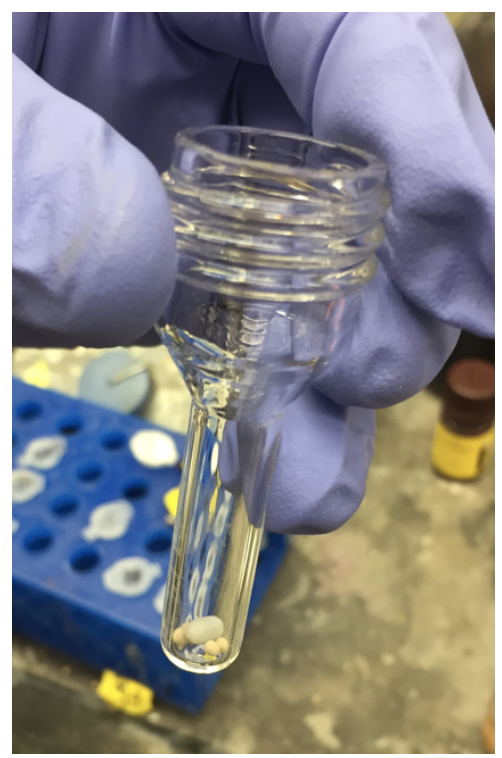

IKA Electrasyn $2 \mathrm{~mL}$ vial charged with small stir bar, and four $4 \AA \mathrm{MS}$

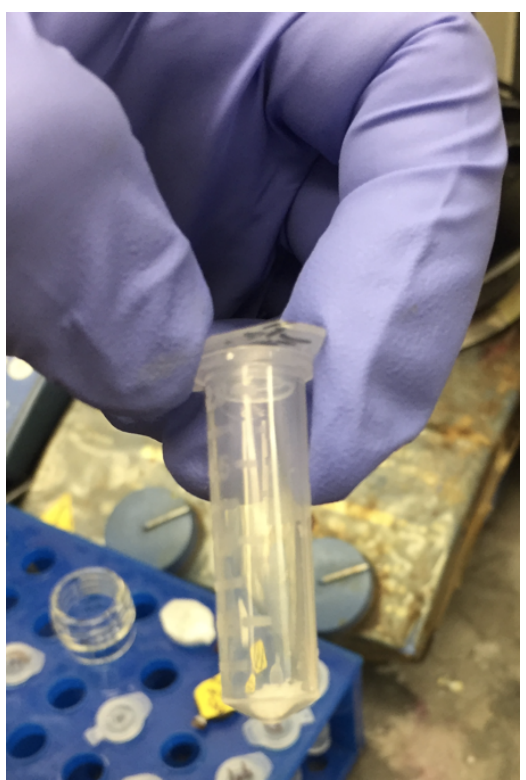

Solid TBAB (16 mg)

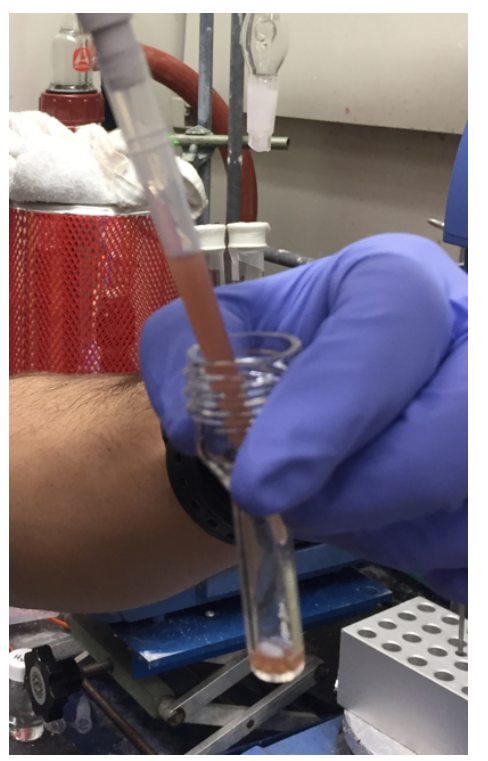

Transferring Ni, Amine, TBAB solution to reaction vessel

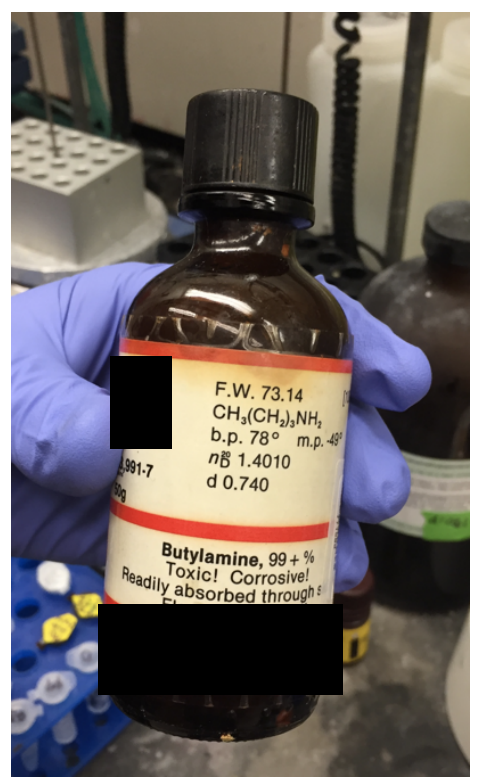

Butylamine

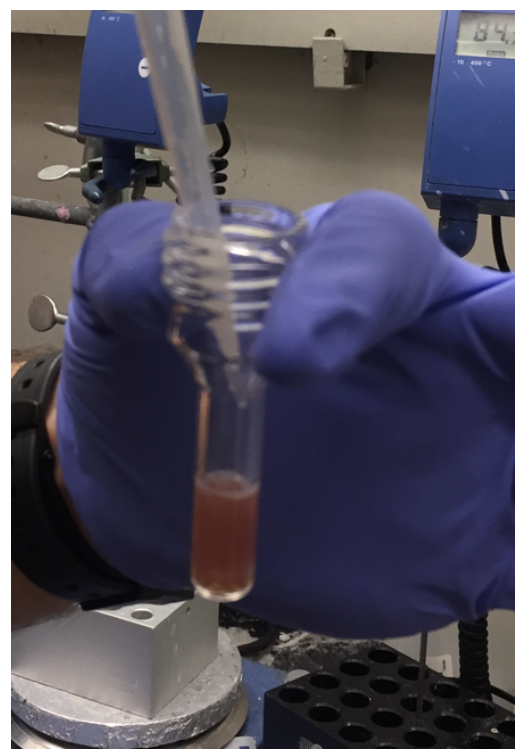

Transferring, by pipette, resin slurry. 


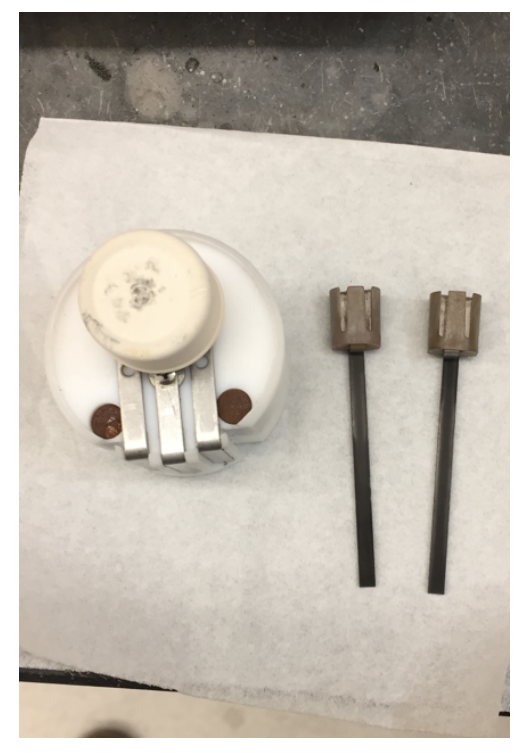

Reaction vessel top, fitted with rubber septum (left) and graphite microelectrodes

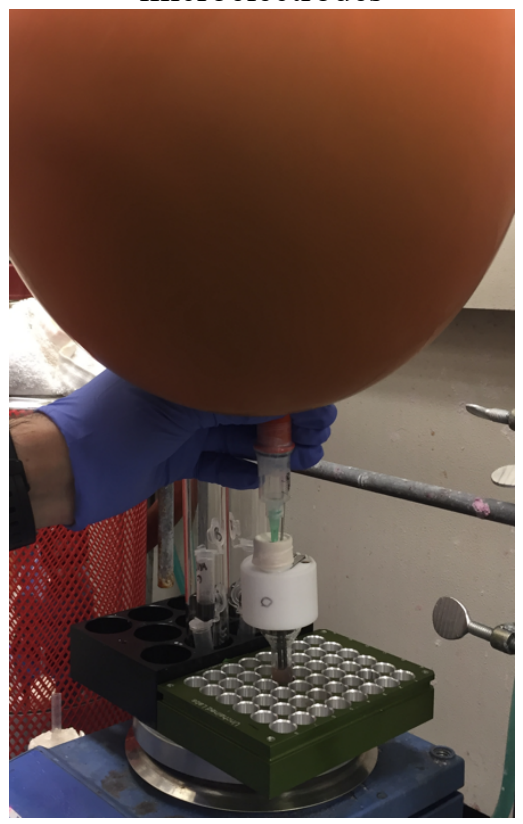

Reaction vessel during degassing procedure

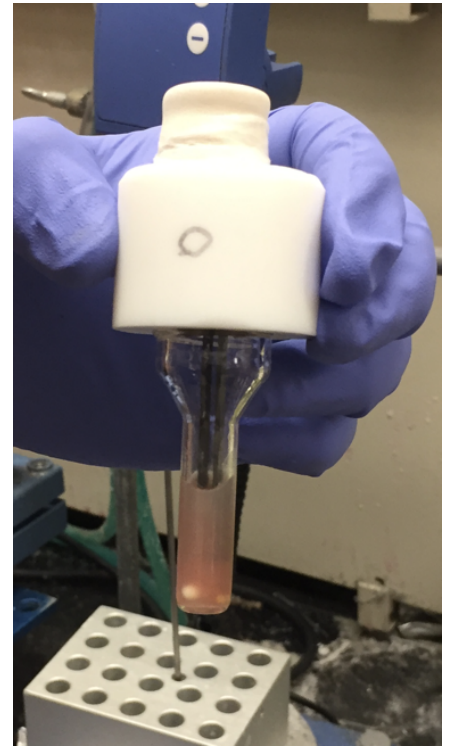

Reaction vessel top with graphite microelectrodes attached

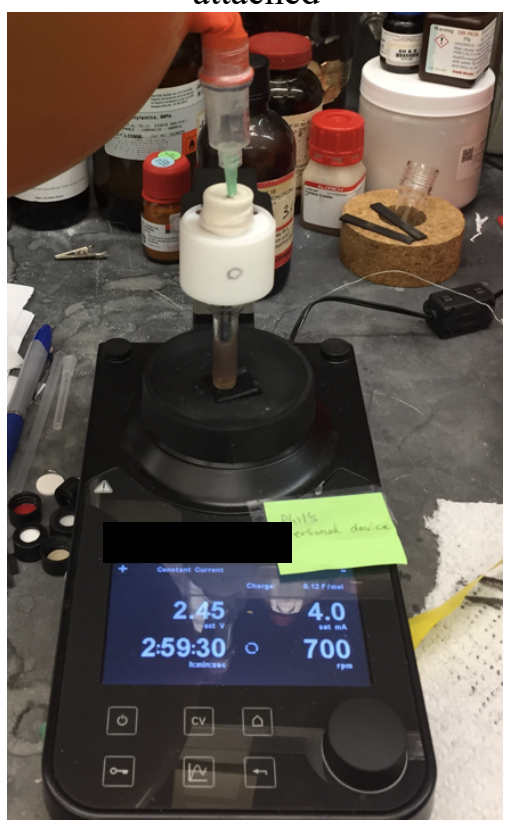

After running the raction for 30 seconds, reaction mixture is still pink

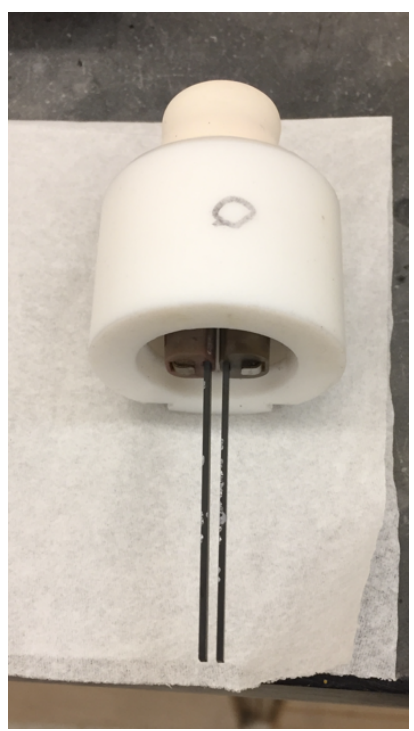

Reaction vessel with top screwed on.

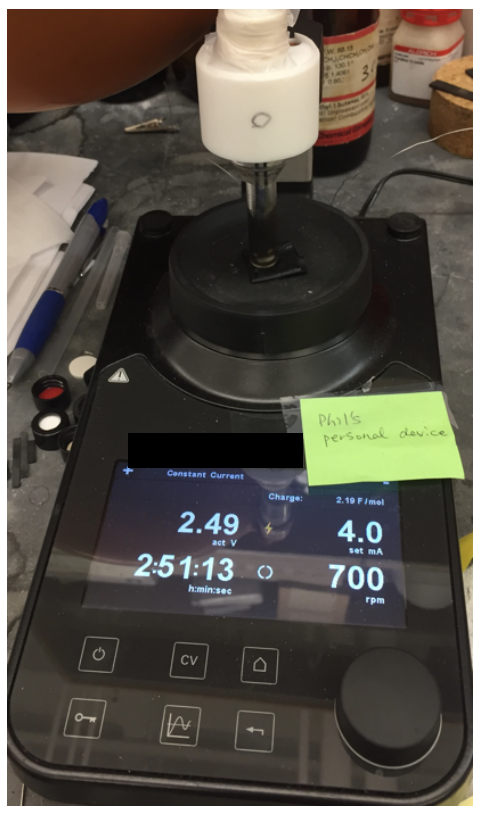

Reaction mixture turns black within 10 minutes of reaction initiation. 


\section{Trouble Shooting: Frequently Asked Questions (e-Aminiation)}

My pink catalyst is not dissolving at $100 \mathrm{mM}$, what should I do?

The pink catalyst is not extremely soluble in DMA, $100 \mathrm{mM}$ is most likely the solubility limit. We have found that when making the solution of pink catalyst vigorous vortexing will get most of it to dissolve but the solution my stay slightly turbid.

During the reaction, solid crashed out and the reaction stopped stirring, what is the problem?

Vigorous stirring is necessary for the success of this reaction. High quality stir bars are required to keep the reaction stirring, old or low quality stir bars will not sufficiently stir the resins, molecular sieve, and solvent mixture.

Why do you use "4" molecular sieves?

Instead of weighing the molecular sieves we found that counting 4 spherical molecular sieves allowed the reaction to proceed without the formation of phenol by-product while minimally effecting stirring.

The voltage is too high, what is wrong?

Dirty electrodes are most likely the cause of the problem. Through washing with dilute $\mathrm{HCl}$, water and acetone should fix this problem.

What happens to the color of this reaction?

The reaction mixture will start out a dirty pink color and after 10-15 minutes of electrochemical treatment it will turn a homogenous black. If this does not happen something probably went wrong and the reaction may not be successful. Towards the end of the three hour reaction, the reaction mixture may turn beige. 


\section{Characterization of $e$-Amination Products}

\section{LC Trace and Mass of 45}

Following General Procedure 6 with 1

Yield: $74 \%$

Exact mass: 5109.98

Triply charged mass [M-3]/3, caluculated: 1702.34; observed 1702.32

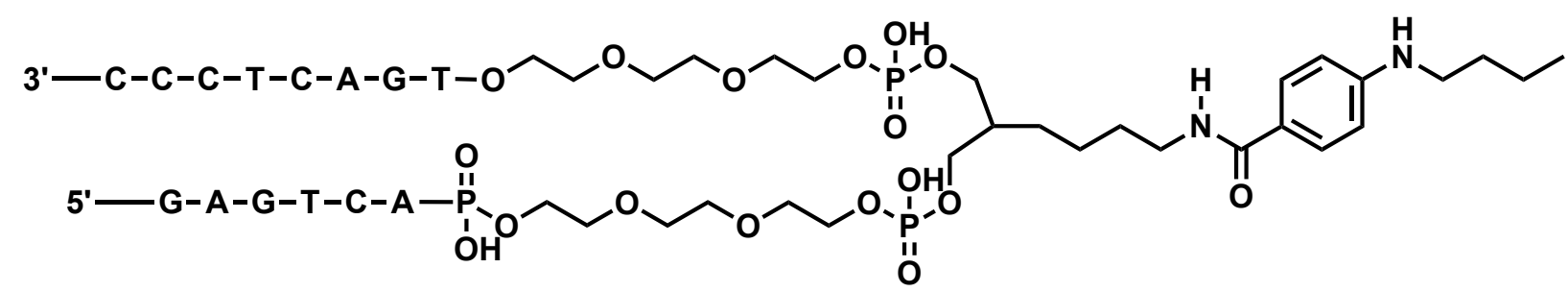
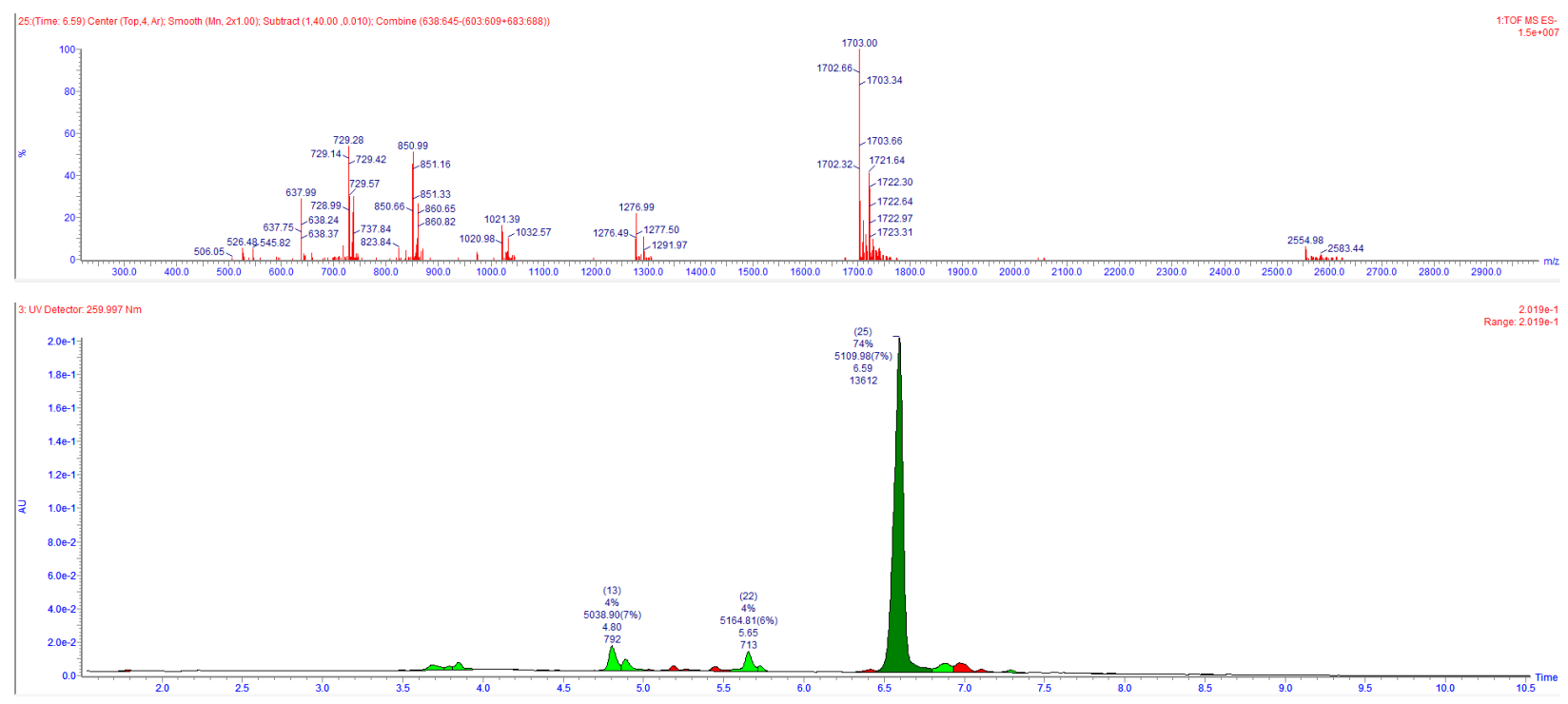


\section{LC Trace and Mass of 46}

Following General Procedure 6 with 1

Yields: 51, 52, 53\%

Average Yields: 52\%

SD: 1

Exact masses: 5109.9

Triply charged masses [M-3]/3, caluculated: 1702.3; observed 1702.32, 1702.32, 1702.32
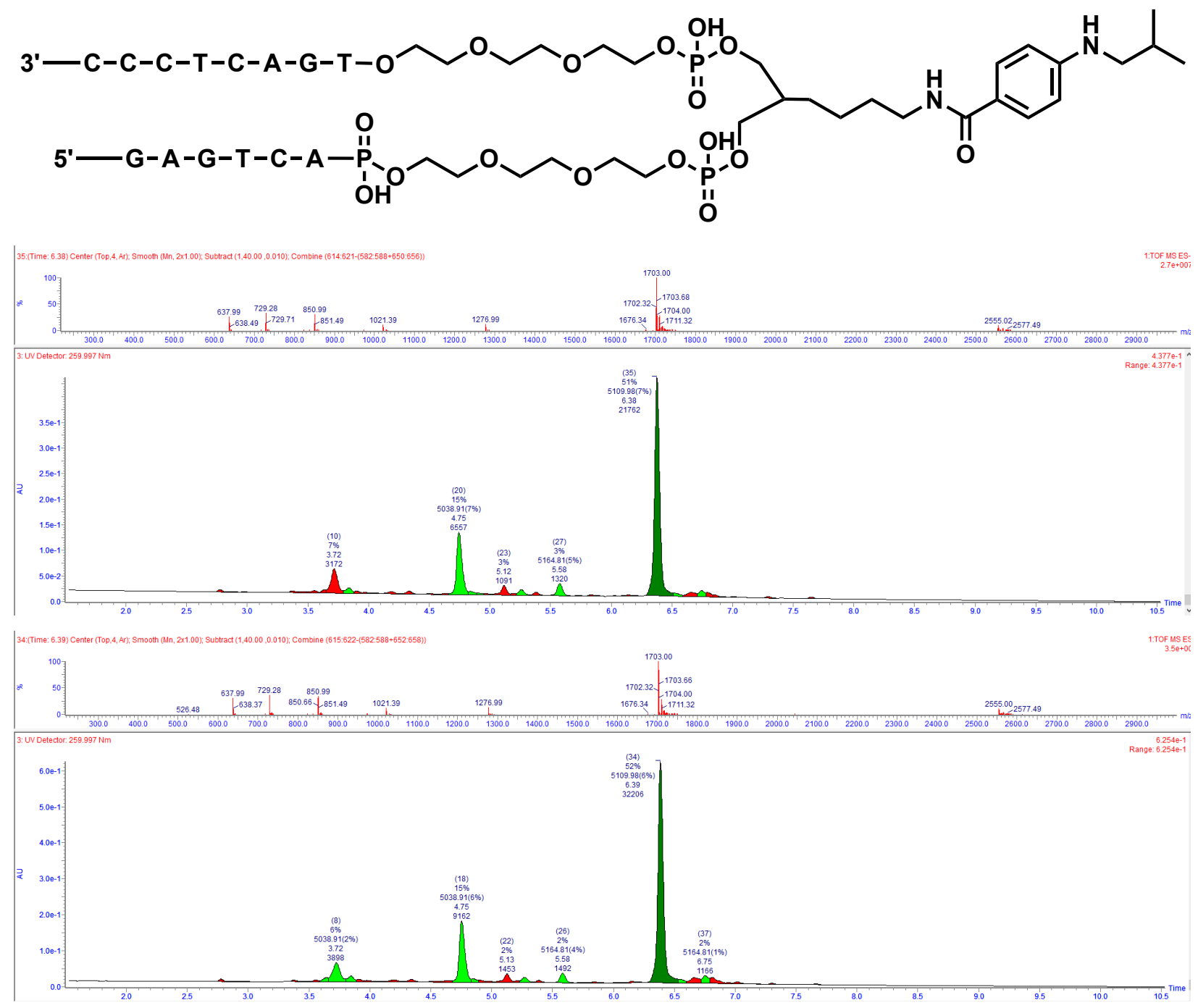


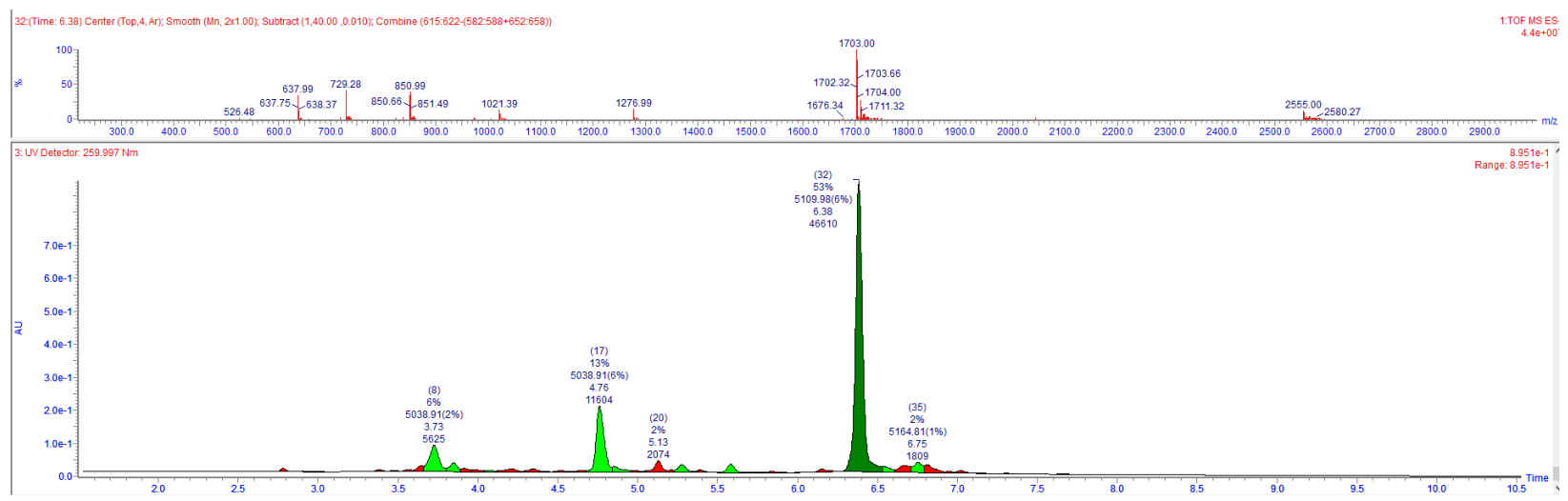




\section{LC Trace and Mass of 47}

Following General Procedure 6 with 1

Yield: $41 \%$

Exact mass: 5111.96

Triply charged mass [M-3]/3, caluculated: 1702.99; observed 1703.00
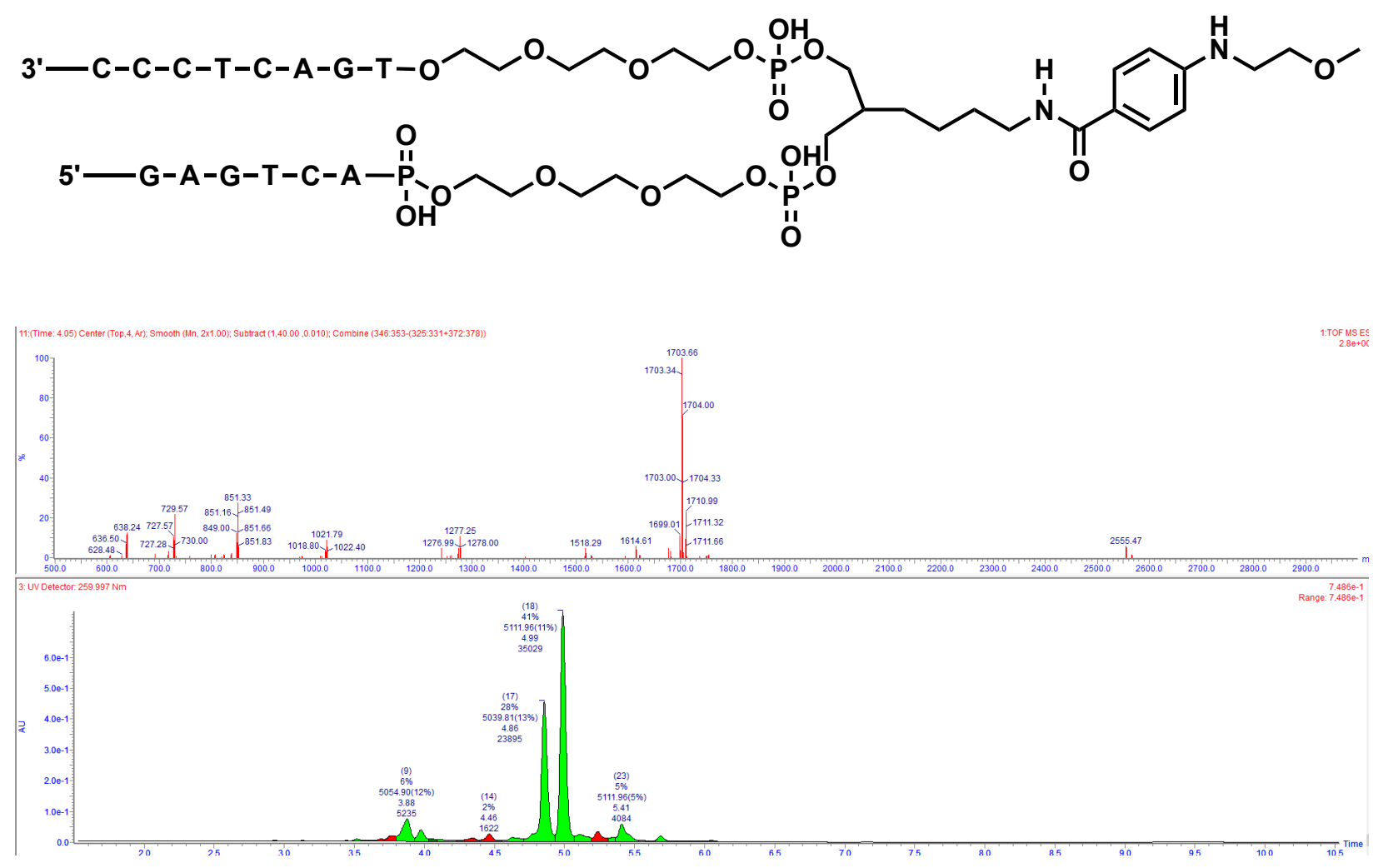


\section{LC Trace and Mass of 48}

Following General Procedure 6 with 1

Yields: $54 \%$

Exact masses: 5121.98

Triply charged masses [M-3]/3, caluculated: 1706.33; observed 1706.33
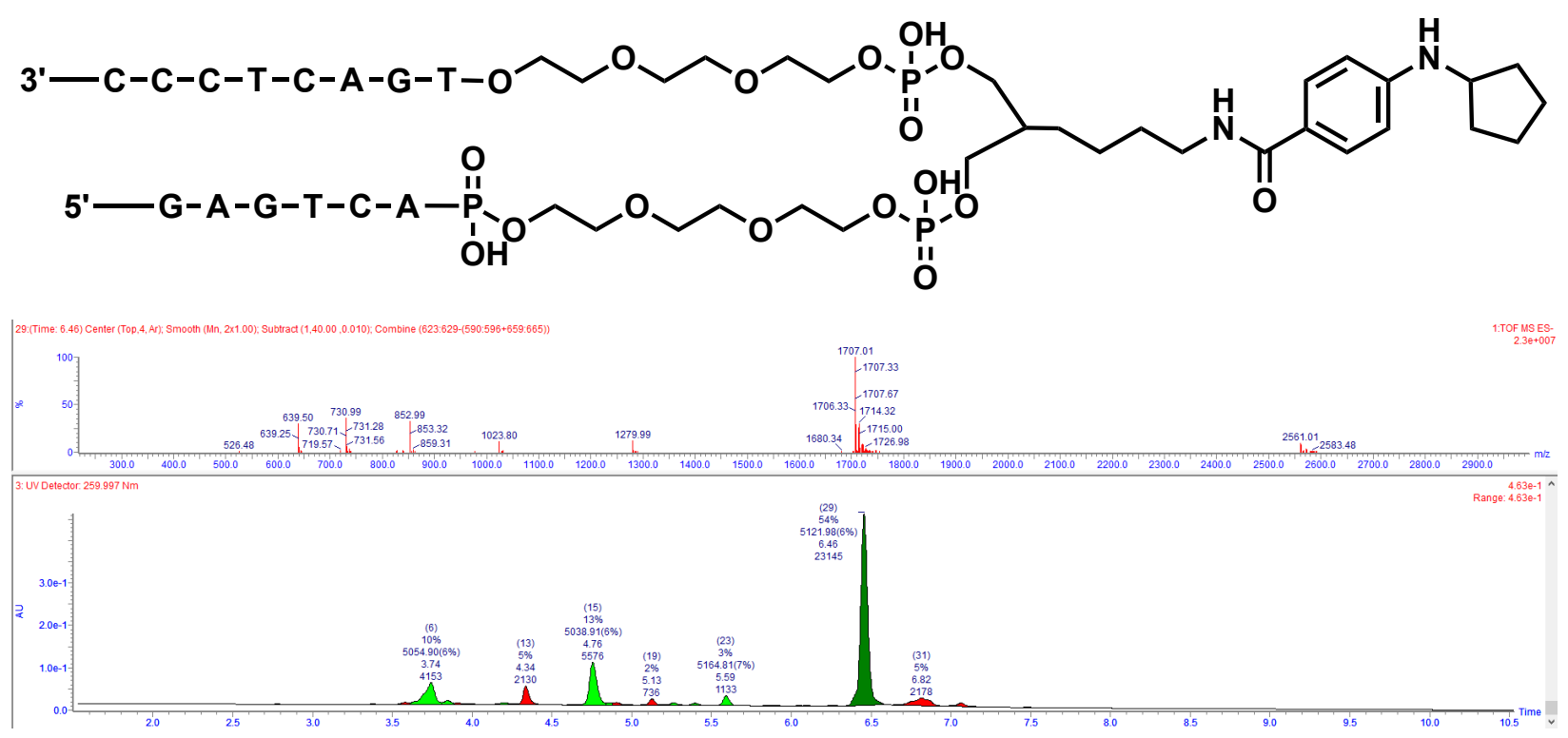


\section{LC Trace and Mass of 49}

Following General Procedure 6 with 1

Yield: $48 \%$

Exact mass: 5136.00

Triply charged mass [M-3]/3, caluculated: 1711.00; observed 1710.99
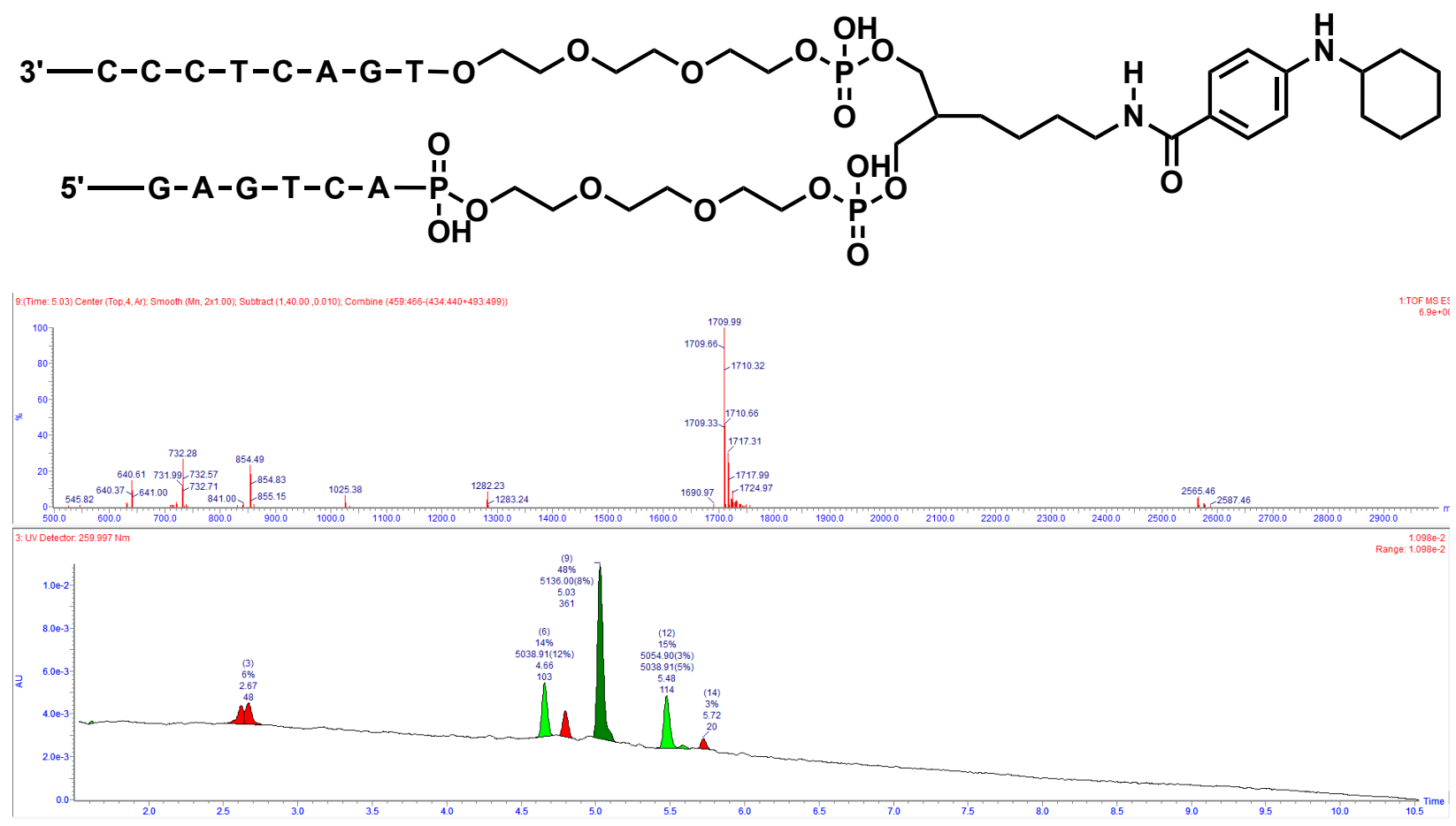


\section{LC Trace and Mass of 50}

Following General Procedure 6 with 1

Yields: $34 \%$

Exact masses: 5219.00

Triply charged masses [M-3]/3, caluculated: 1738.66; observed 1738.66
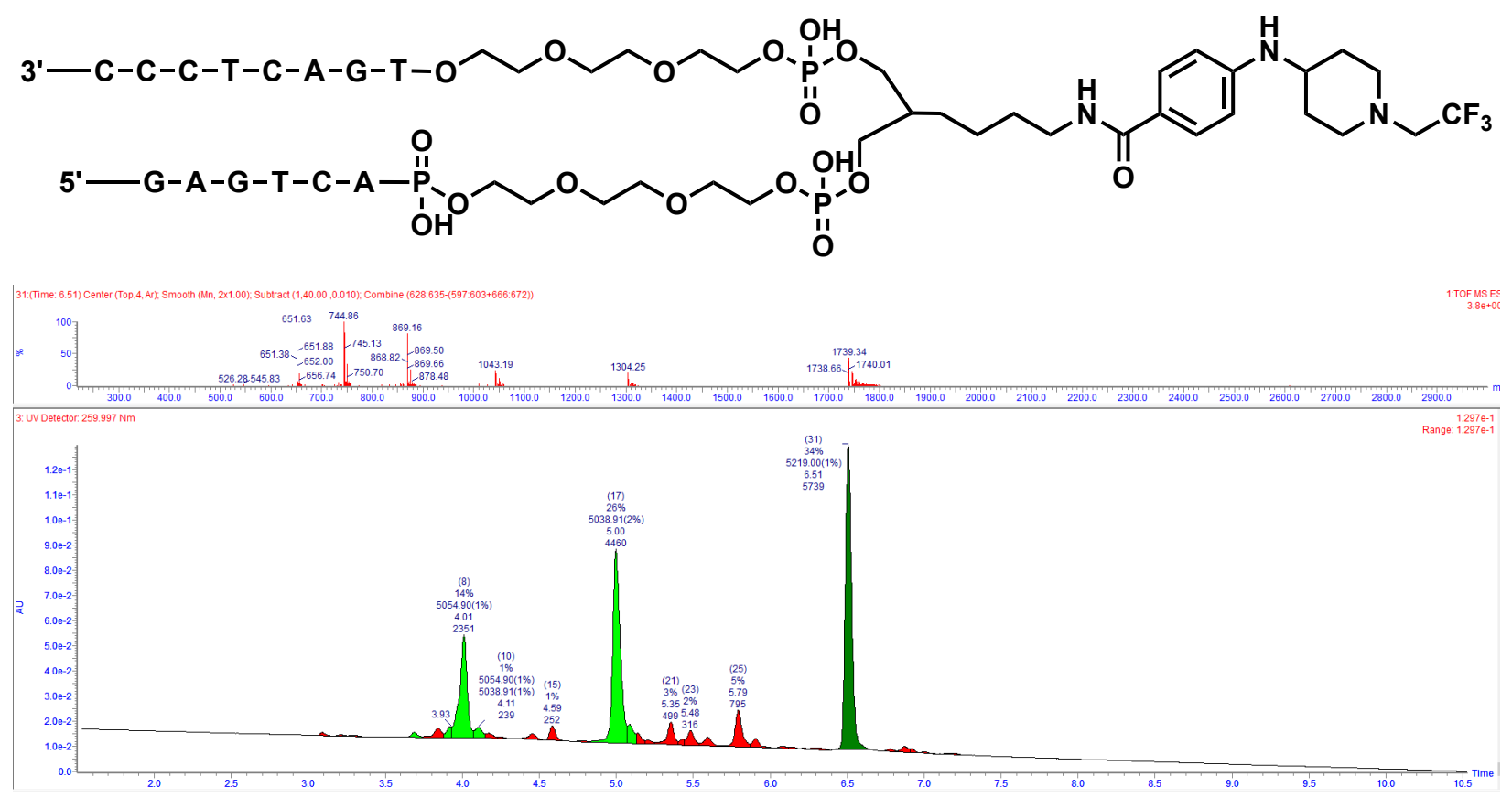


\section{LC Trace and Mass of 51}

Following General Procedure 6 with 1 and 100 mM DBU.

Yield: $31 \%$

Exact mass: 5182.00

Triply charged mass [M-3]/3, caluculated: 1726.33 observed 1726.33
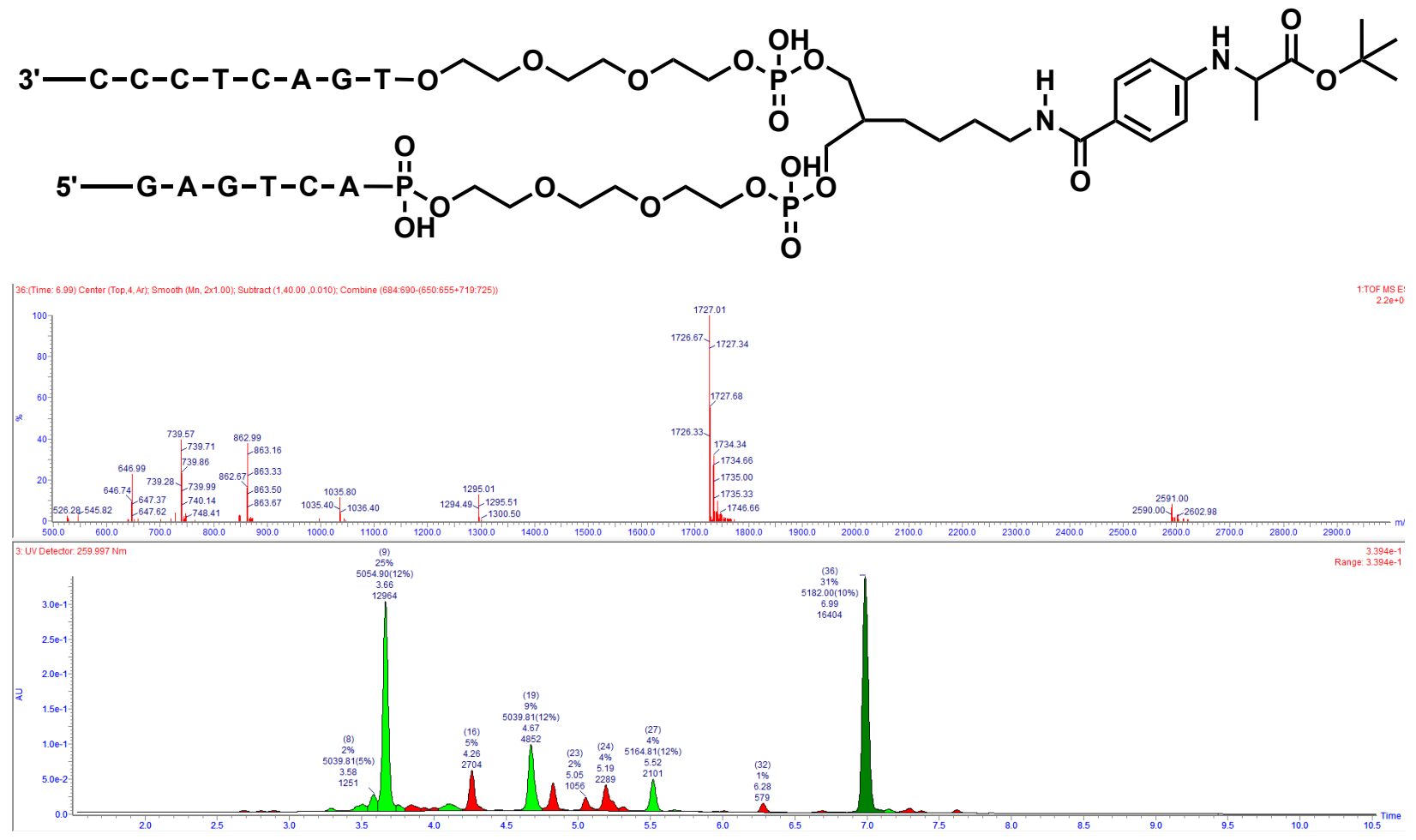


\section{LC Trace and Mass of 52}

Following General Procedure 6 with 1

Yield: $55 \%$

Exact mass: 5136.00

Triply charged mass [M-3]/3, caluculated: 1711.00; observed 1710.38
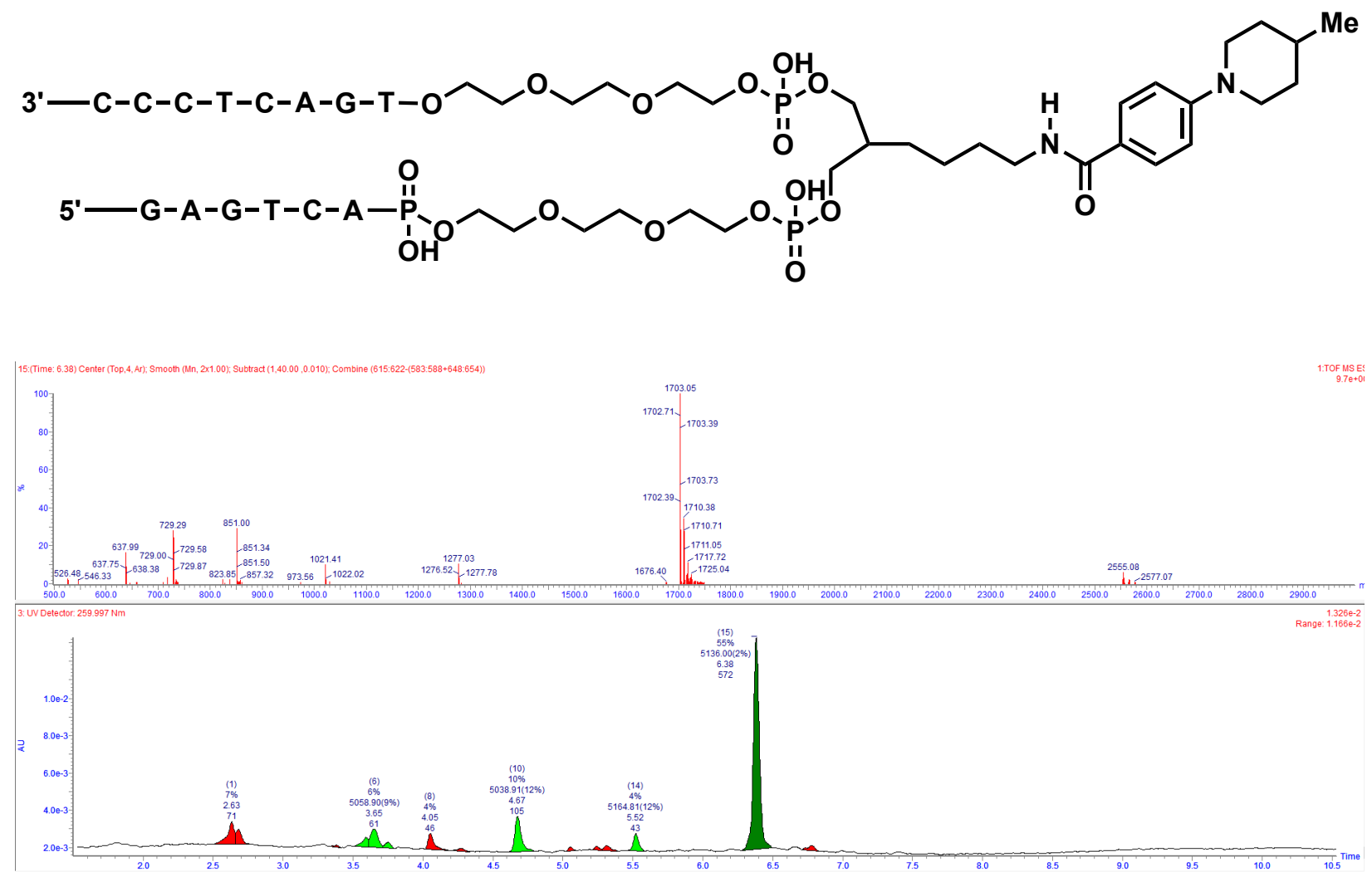


\section{LC Trace and Mass of 53}

Following General Procedure 6 with 1

Yield: $46 \%$

Exact mass: 5137.98

Triply charged mass [M-3]/3, caluculated: 1711.66; observed 1711.66
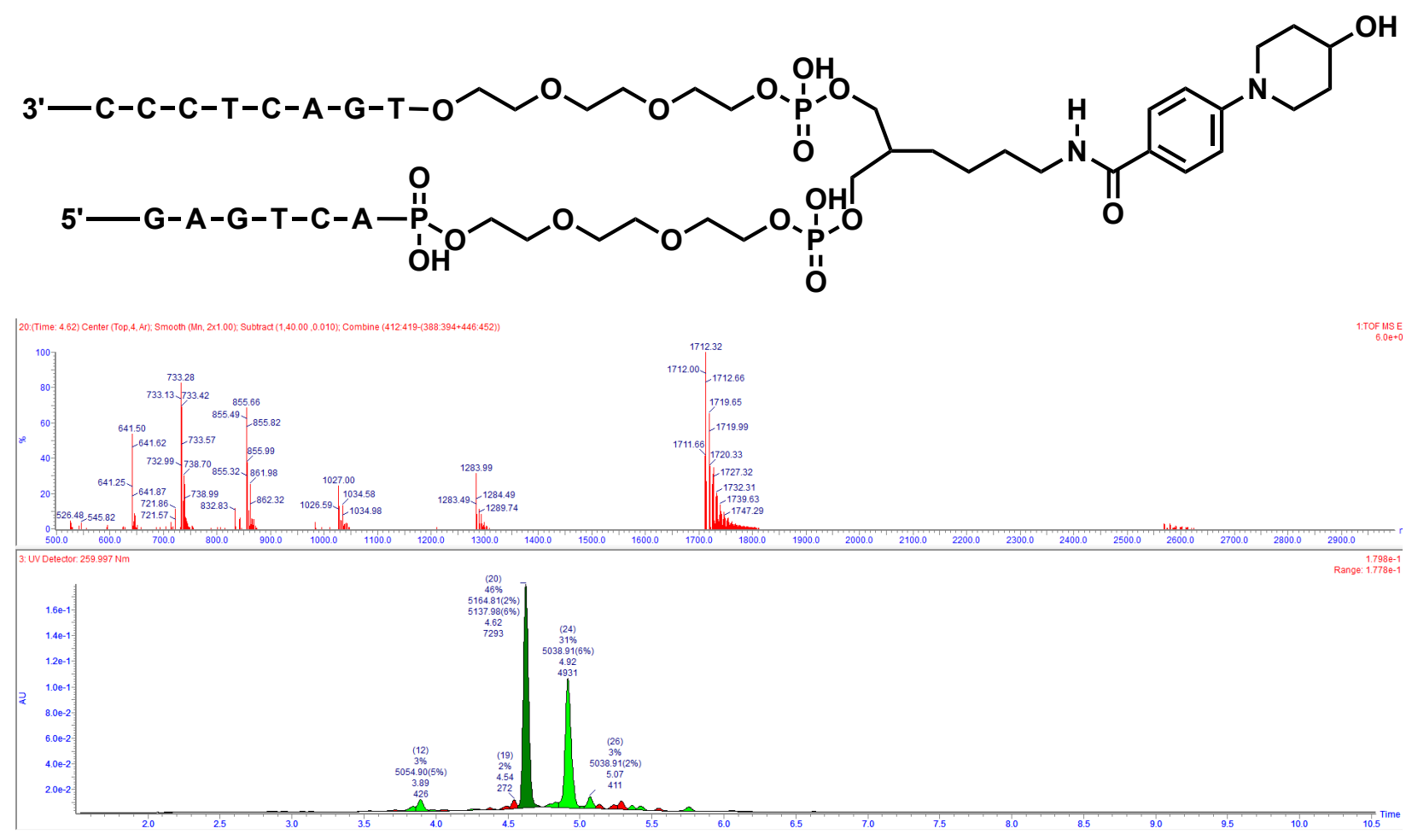


\section{LC Trace and Mass of 54}

Following General Procedure 6 with 1

Yields: $43 \%$

Exact masses: 5179.99

Triply charged masses [M-3]/3, caluculated: 1725.66; observed 1725.65
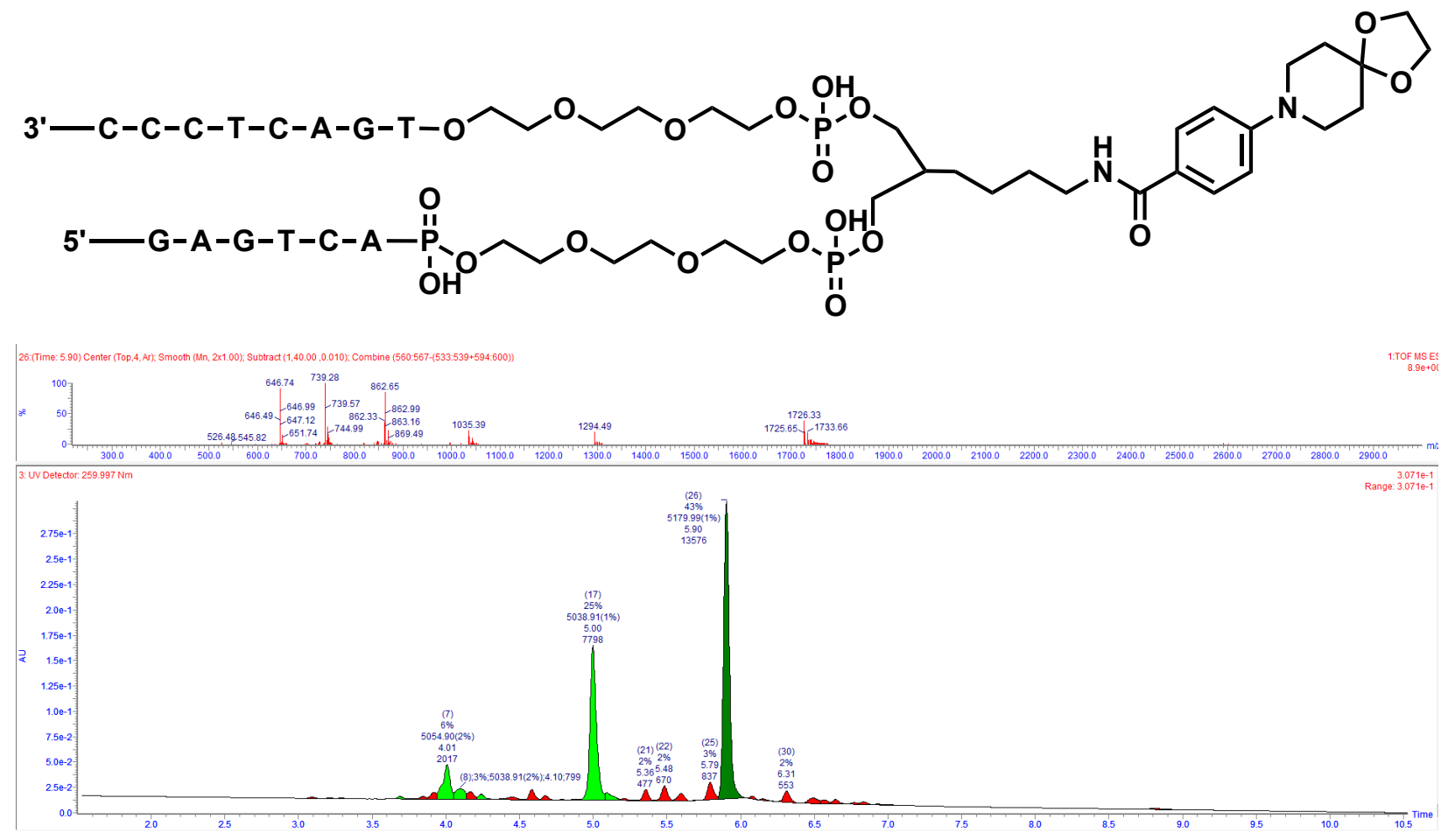


\section{LC Trace and Mass of 55}

Following General Procedure XX with $\mathbf{1}$

Analysis method: $\mathbf{X X}$

Yield: 51\%

Exact mass: 5123.96

Triply charged mass [M-3]/3, caluculated: 1706.98; observed 1707.00
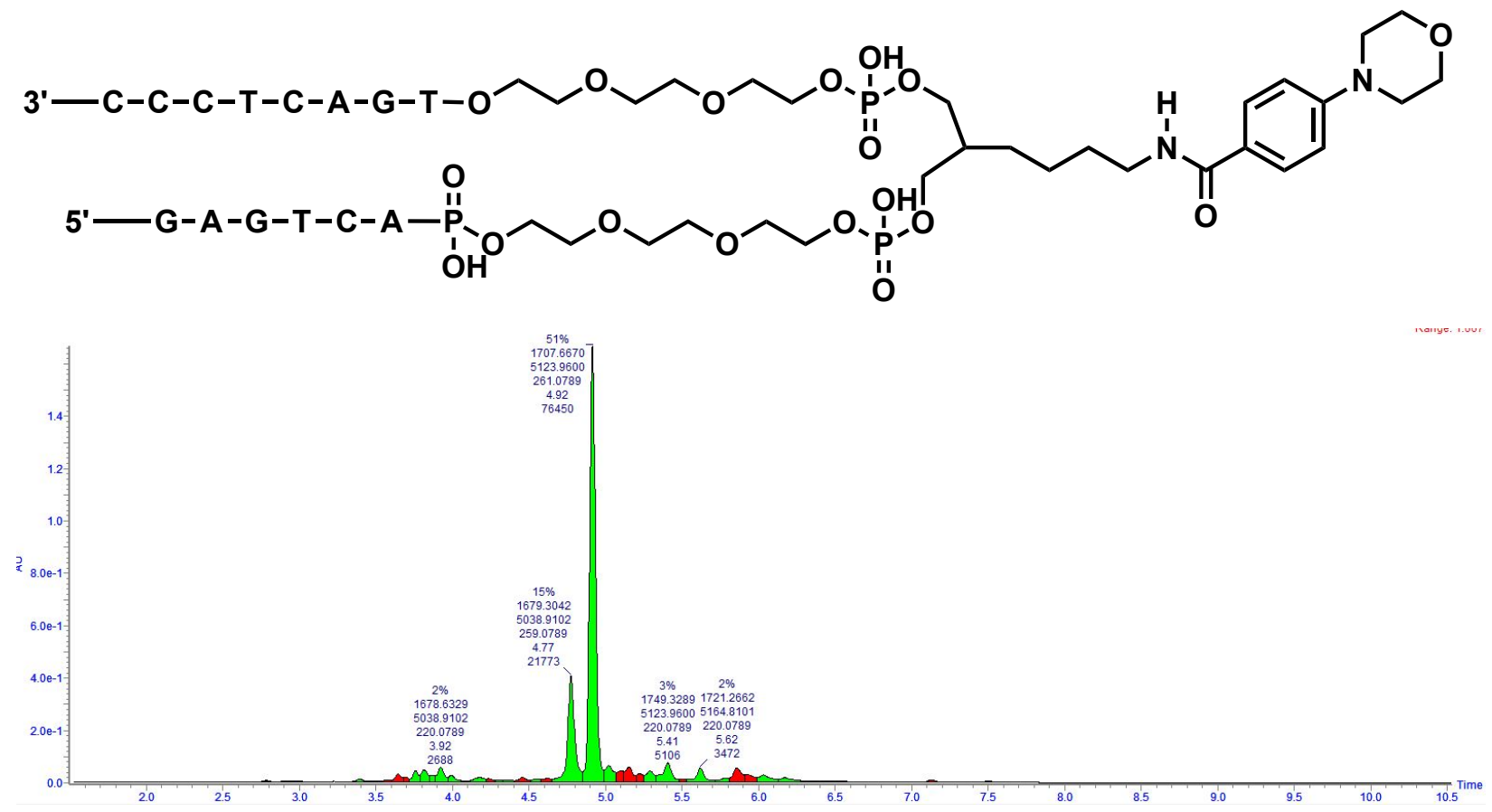


\section{LC Trace and Mass of 56}

Following General Procedure 6 with 1

Yield: $49 \%$

Exact mass: 5142.92

Triply charged mass [M-3]/3, caluculated: 1712.97; observed 1712.31
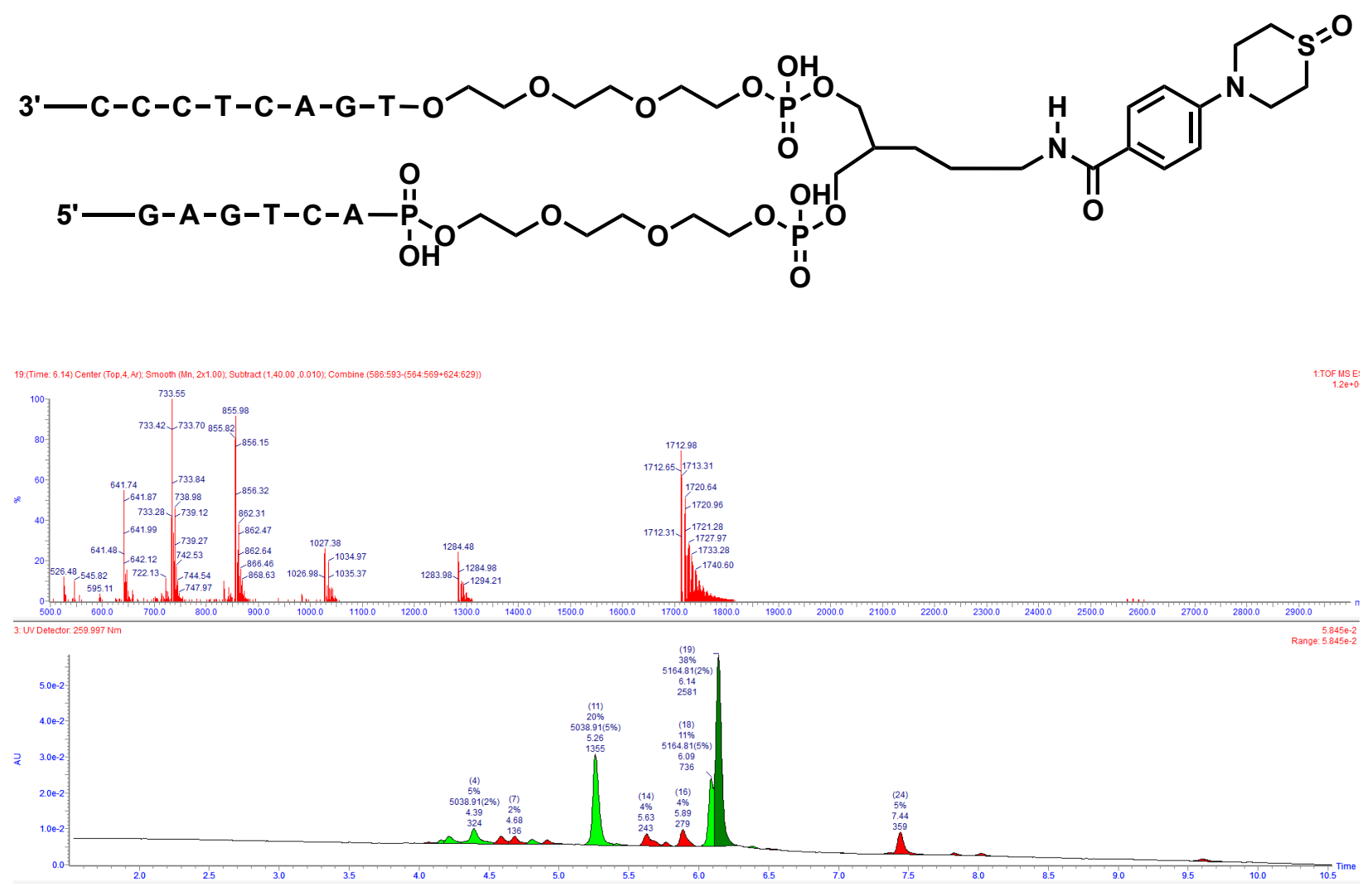


\section{LC Trace and Mass of 57}

Following General Procedure 6 with 1

Yields: $47 \%$

Exact masses: 5248.03

Triply charged masses [M-3]/3, caluculated: 1748.34; observed 1748.34

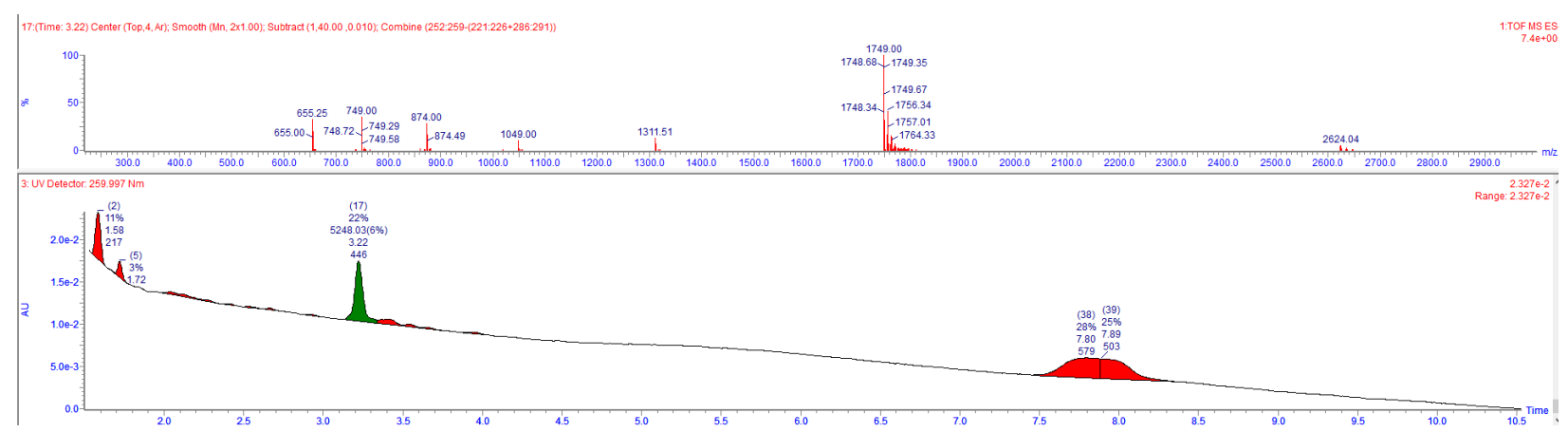




\section{LC Trace and Mass of 58}

Following General Procedure 6 with 1

Yield: $63 \%$

Exact mass: 5093.95

Triply charged mass [M-3]/3, caluculated: 1696.98; observed 1696.99

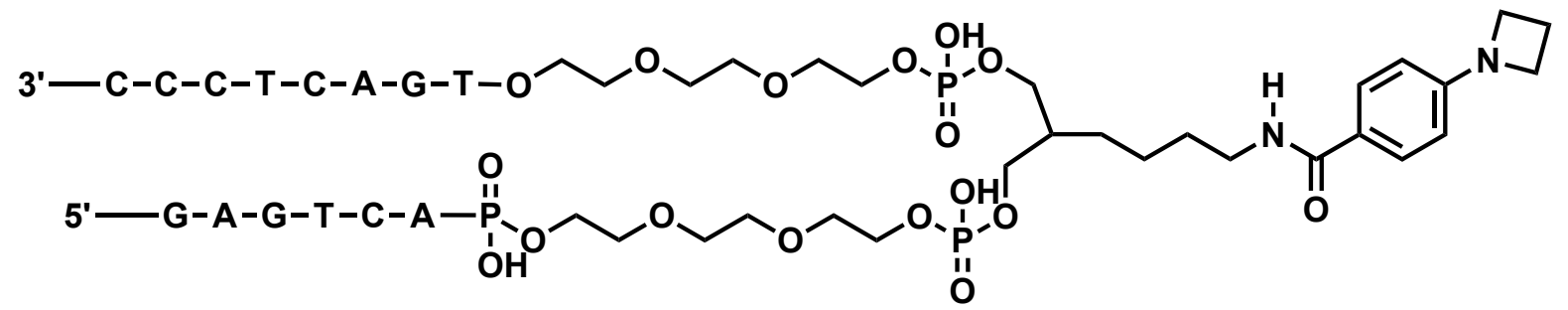

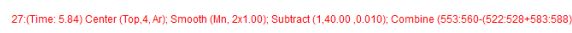
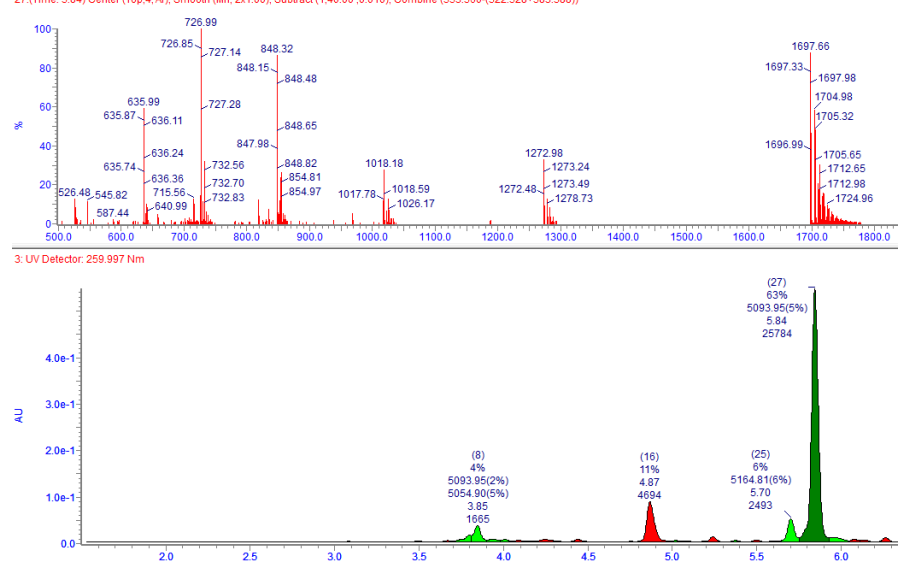


\section{LC Trace and Mass of 59}

Following General Procedure 6 with 1

Yield: $26 \%$

Exact mass: 5107.97

Triply charged mass [M-3]/3, caluculated: 1701.65; observed 1701.28
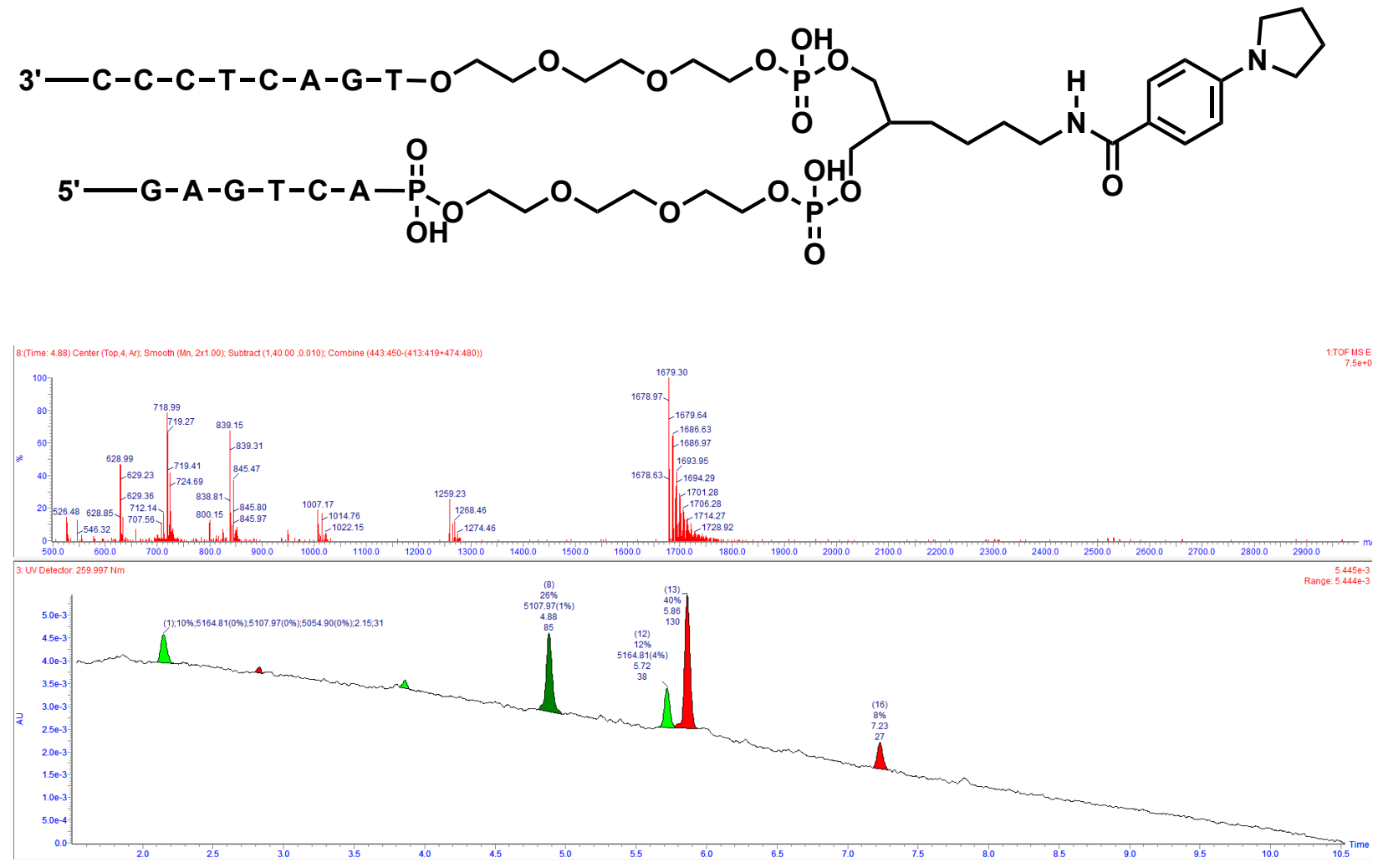


\section{LC Trace and Mass of 60}

Following General Procedure 6 with 1

Yield: $47 \%$

Exact mass: 5121.98

Triply charged mass [M-3]/3, caluculated: 1706.32; observed 1706.31
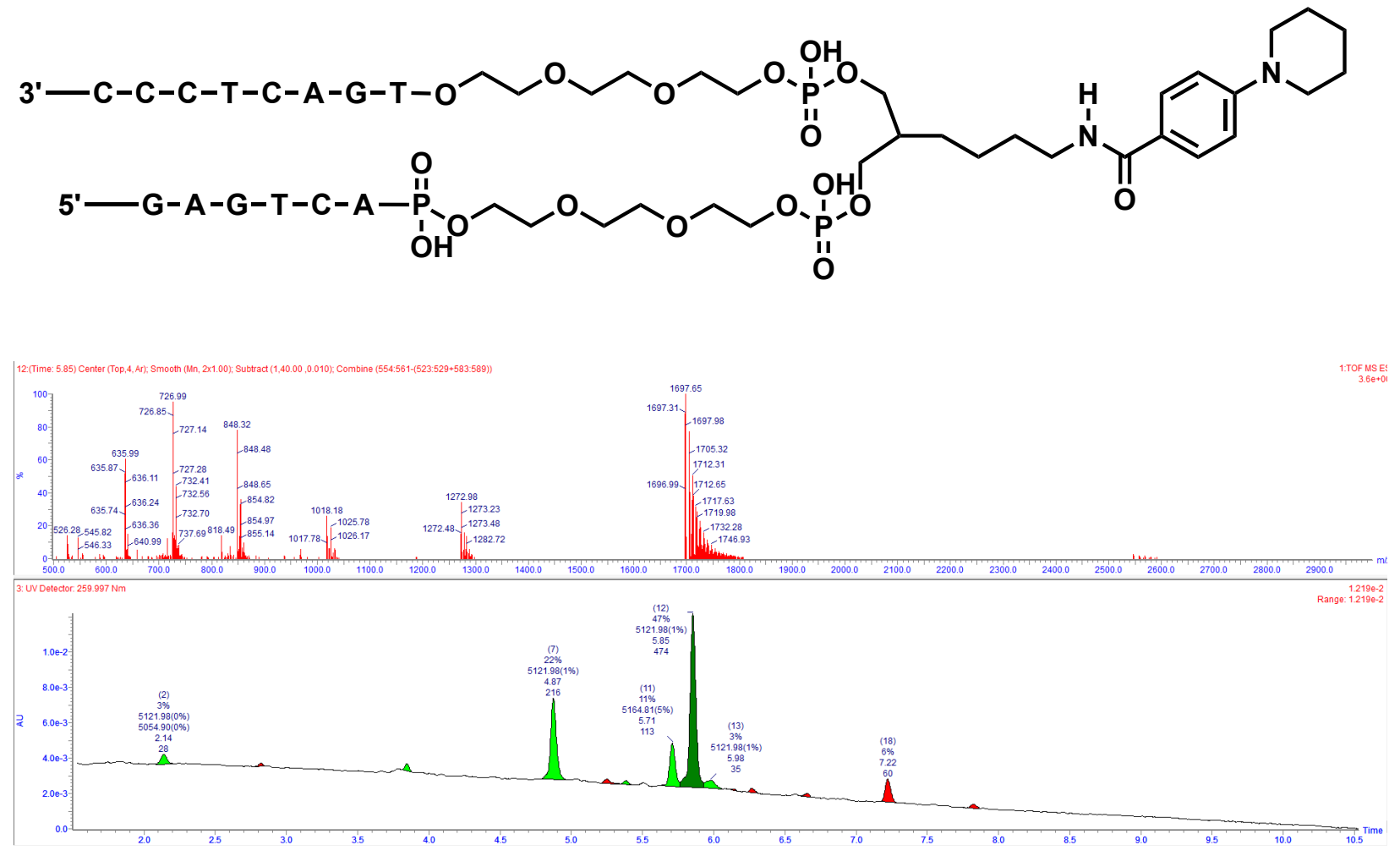


\section{LC Trace and Mass of 61}

Following General Procedure 6 with 1

Yield: $31 \%$

Exact mass: 5136.00

Triply charged mass [M-3]/3, caluculated: 1711.00; observed 1711.00
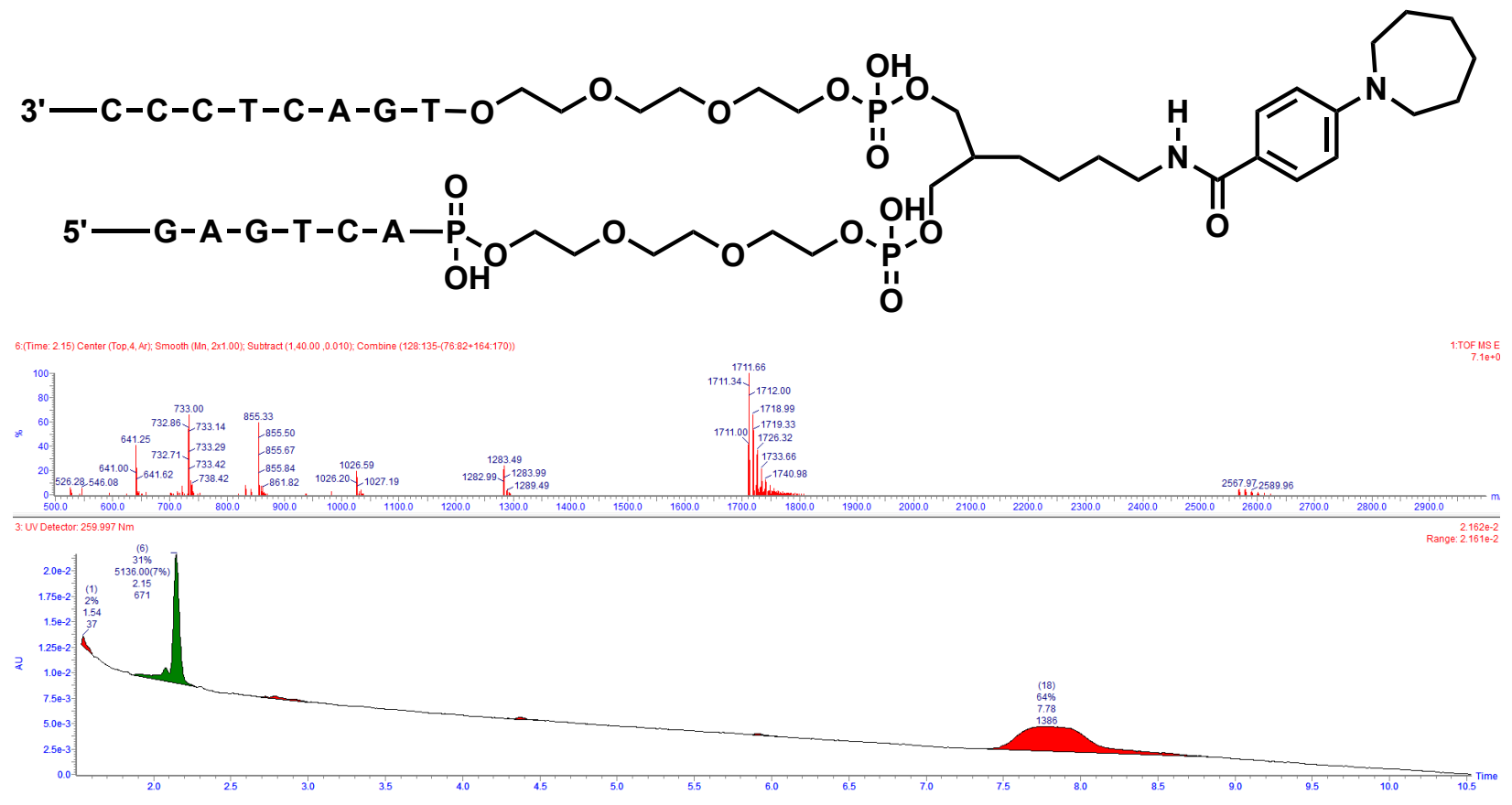


\section{LC Trace and Mass of 62}

Following General Procedure 6 with 1 and 100 mM DBU.

Yield: $18 \%$

Exact mass: 5130.95

Triply charged mass [M-3]/3, caluculated: 1709.27; observed 1709.31
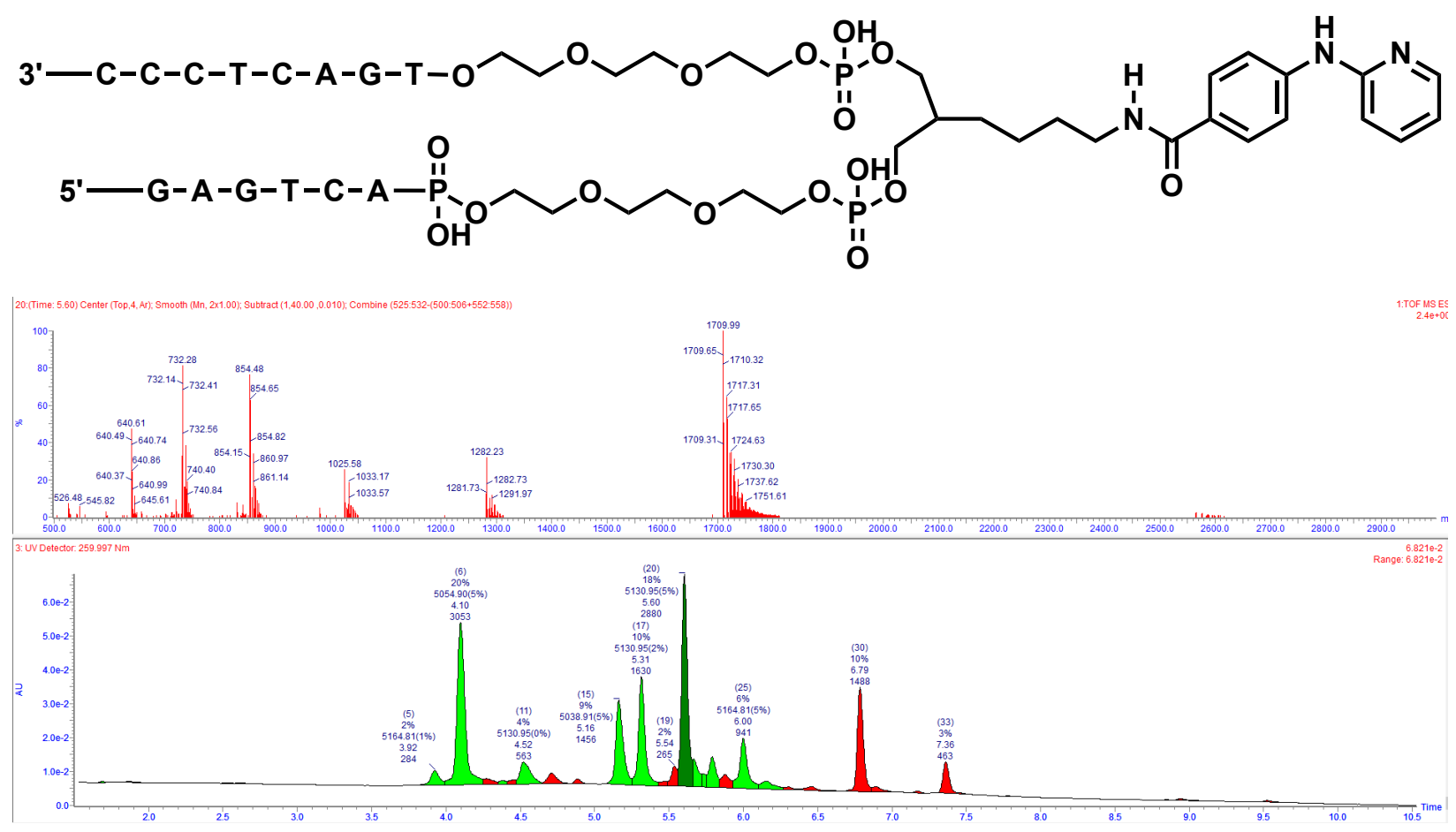


\section{LC Trace and Mass of 63}

Following General Procedure 6 with 1 and 100 mM DBU.

Yield: $25 \%$

Exact mass: 5170.95

Triply charged mass [M-3]/3, caluculated: 1722.65; observed 1722.61
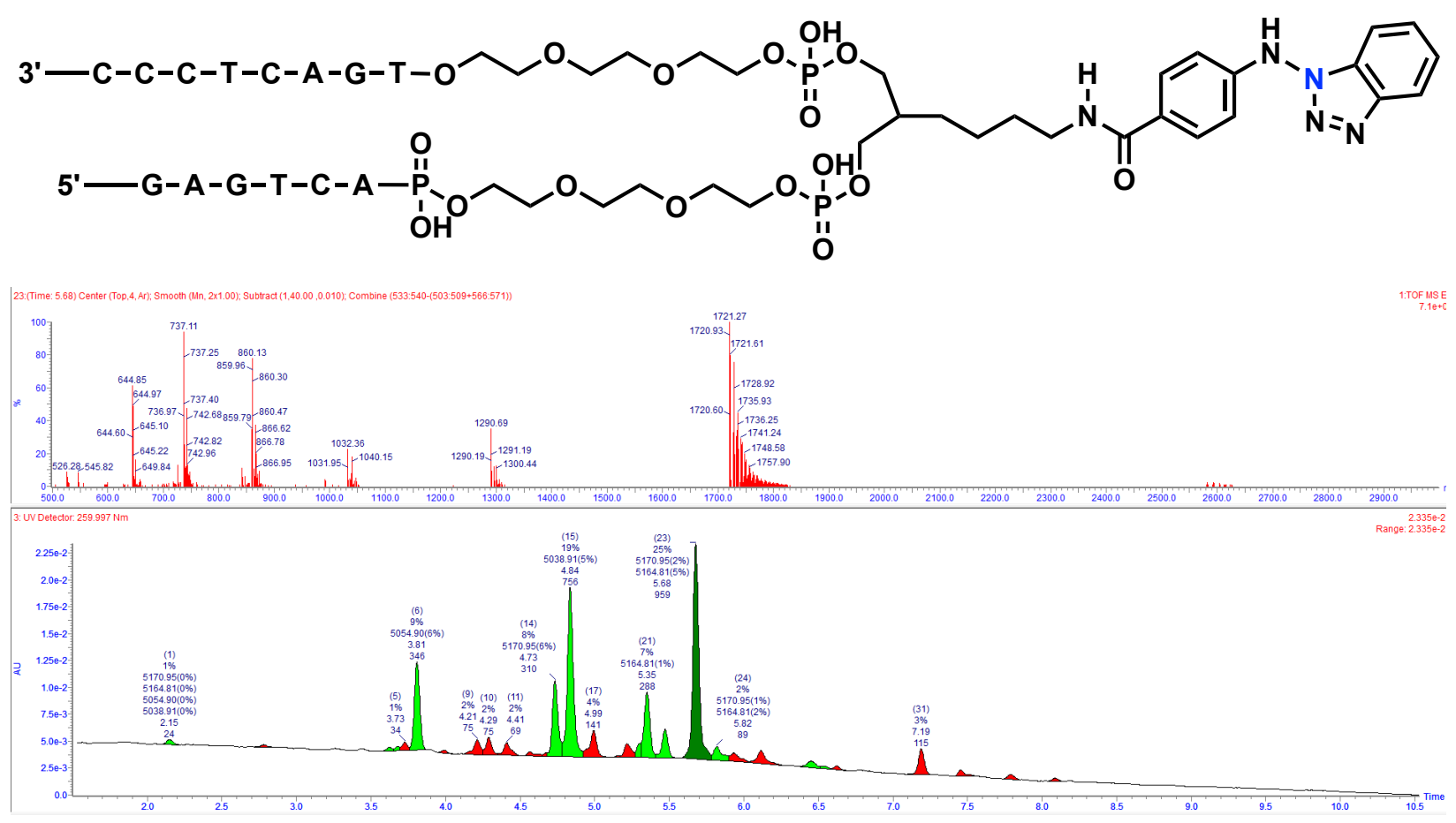


\section{LC Trace and Mass of 64}

Following General Procedure 6 with 1 and 100 mM DBU.

Yield: $28 \%$

Exact mass: 5119.94

Triply charged mass [M-3]/3, caluculated: 1705.; observed 1707.00
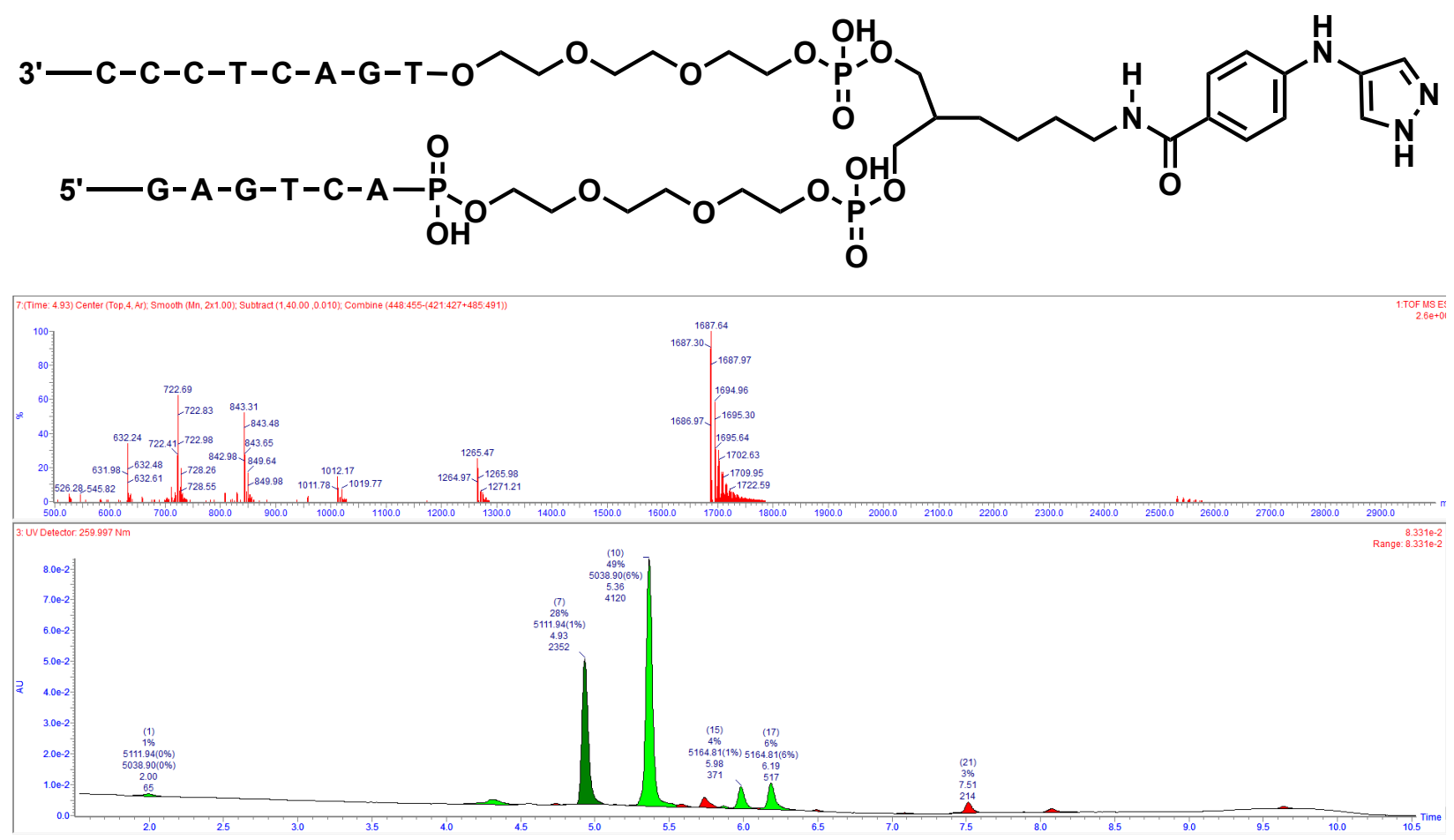


\section{LC Trace and Mass of 65}

Following General Procedure 6 with 1 and 100 mM DBU.

Yield: $21 \%$

Exact mass: 5121.95

Triply charged mass [M-3]/3, caluculated: 1706.65; observed 1706.31
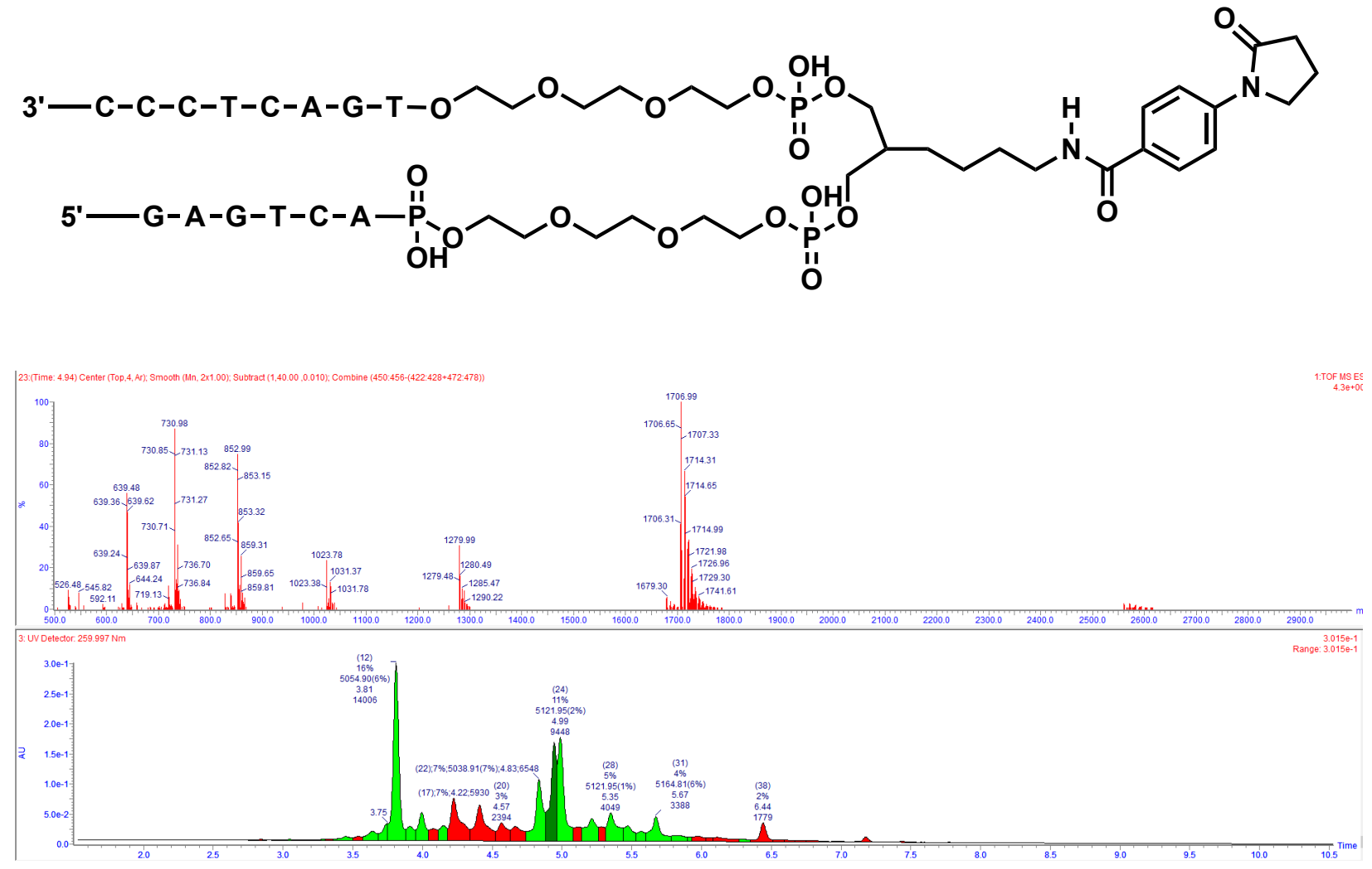


\section{FAILED PIPERZINE BASED REACTIONS}

\section{LC Trace and Mass}

Following General Procedure 6 with 1

Yield: $16 \%$

Exact mass: 5136.99

Triply charged mass [M-3]/3, caluculated: 1711.33; observed 1711.32
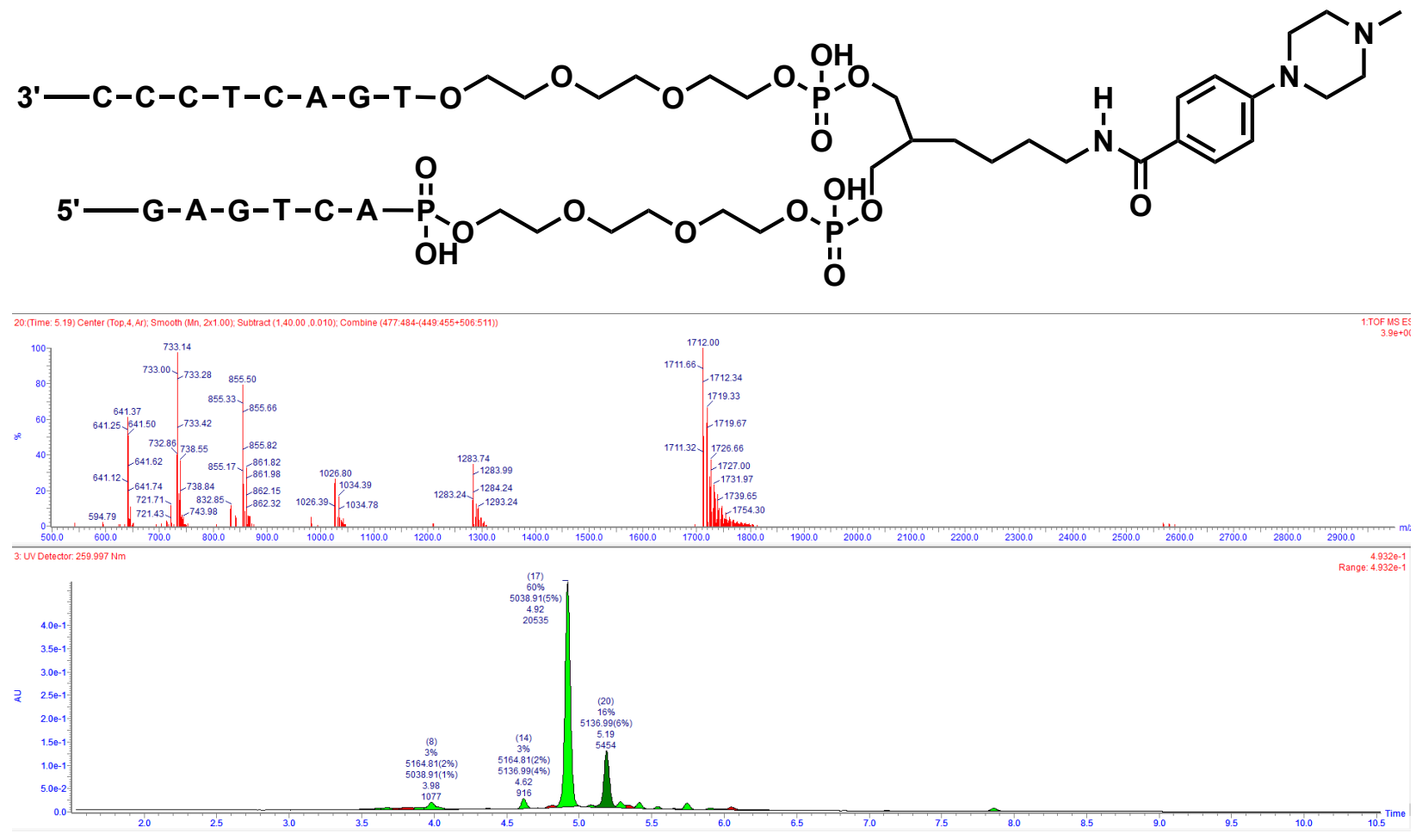


\section{LC Trace and Mass}

Following General Procedure 6 with 1

Yield: $12 \%$

Exact mass: 5223.03

Triply charged mass [M-3]/3, caluculated: 1740.01; observed 1740.01
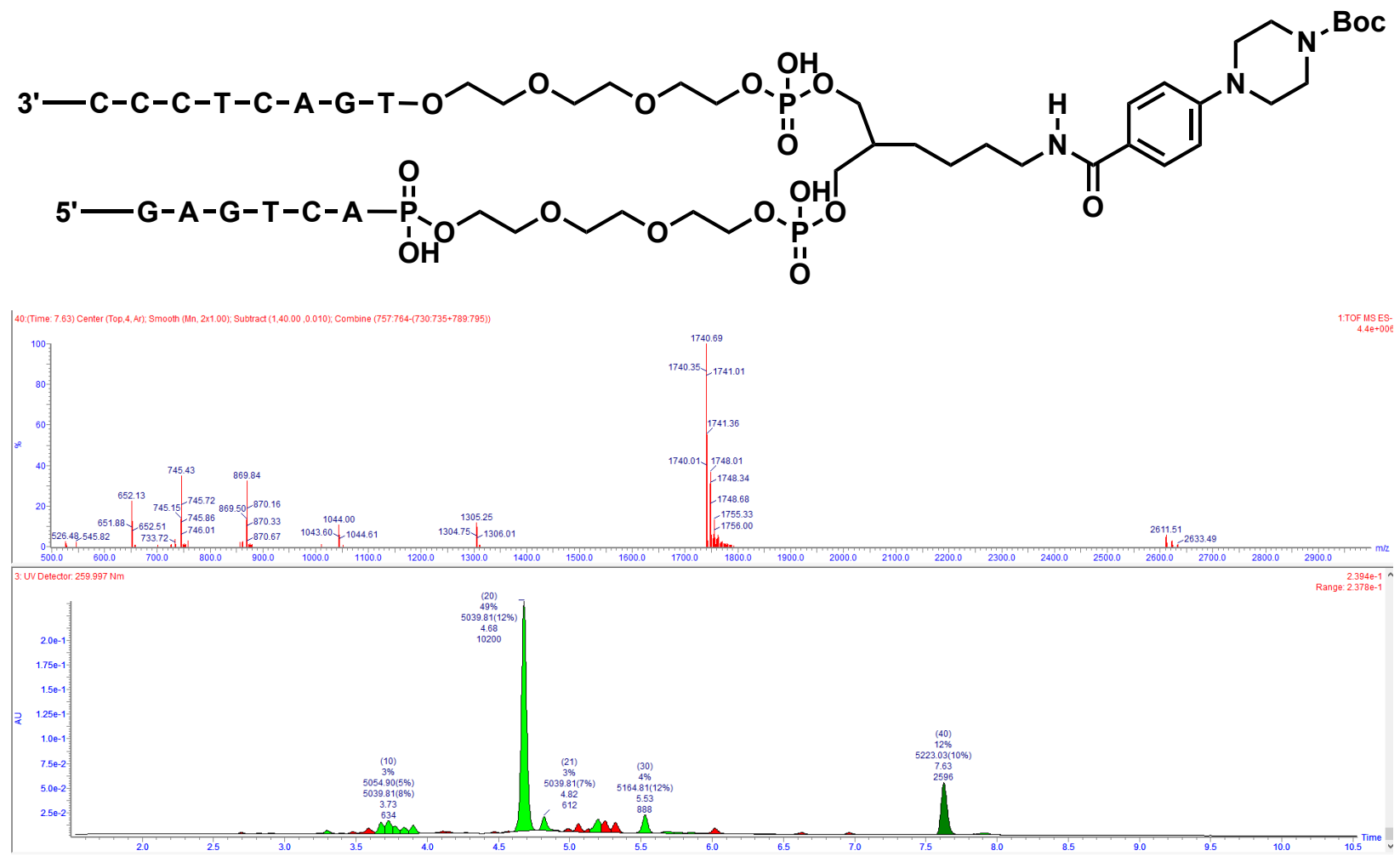


\section{LC Trace and Mass}

Following General Procedure 6 with 1

Yield: $7 \%$

Exact mass: 5200.00

Triply charged mass [M-3]/3, caluculated: 1732.33; observed 1732.33
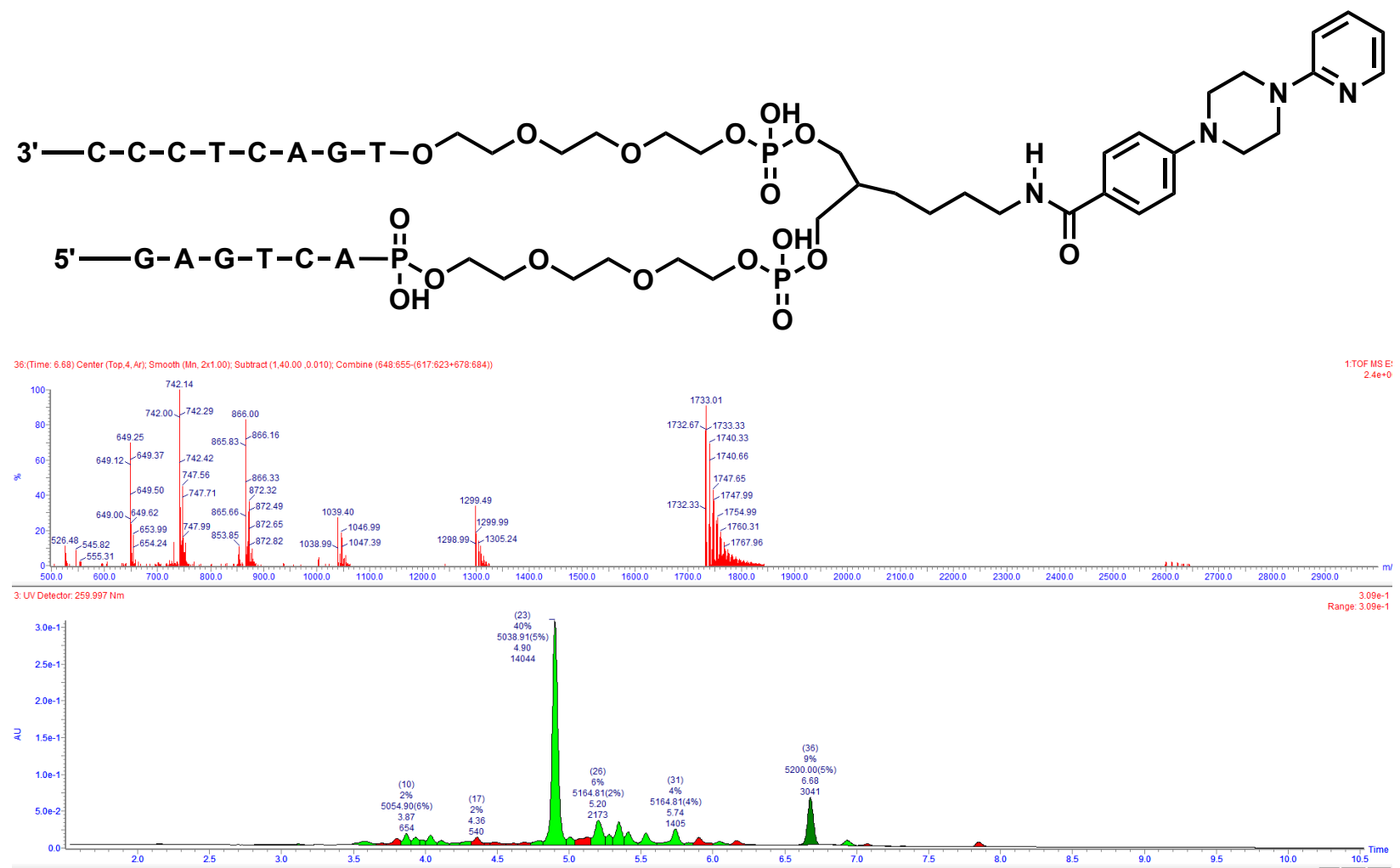


\section{LC Trace and Mass}

Following General Procedure 6 with 1

Yield: 7\%

Exact mass: 5237.05

Triply charged mass [M-3]/3, caluculated: 1744.68; observed 1744.67
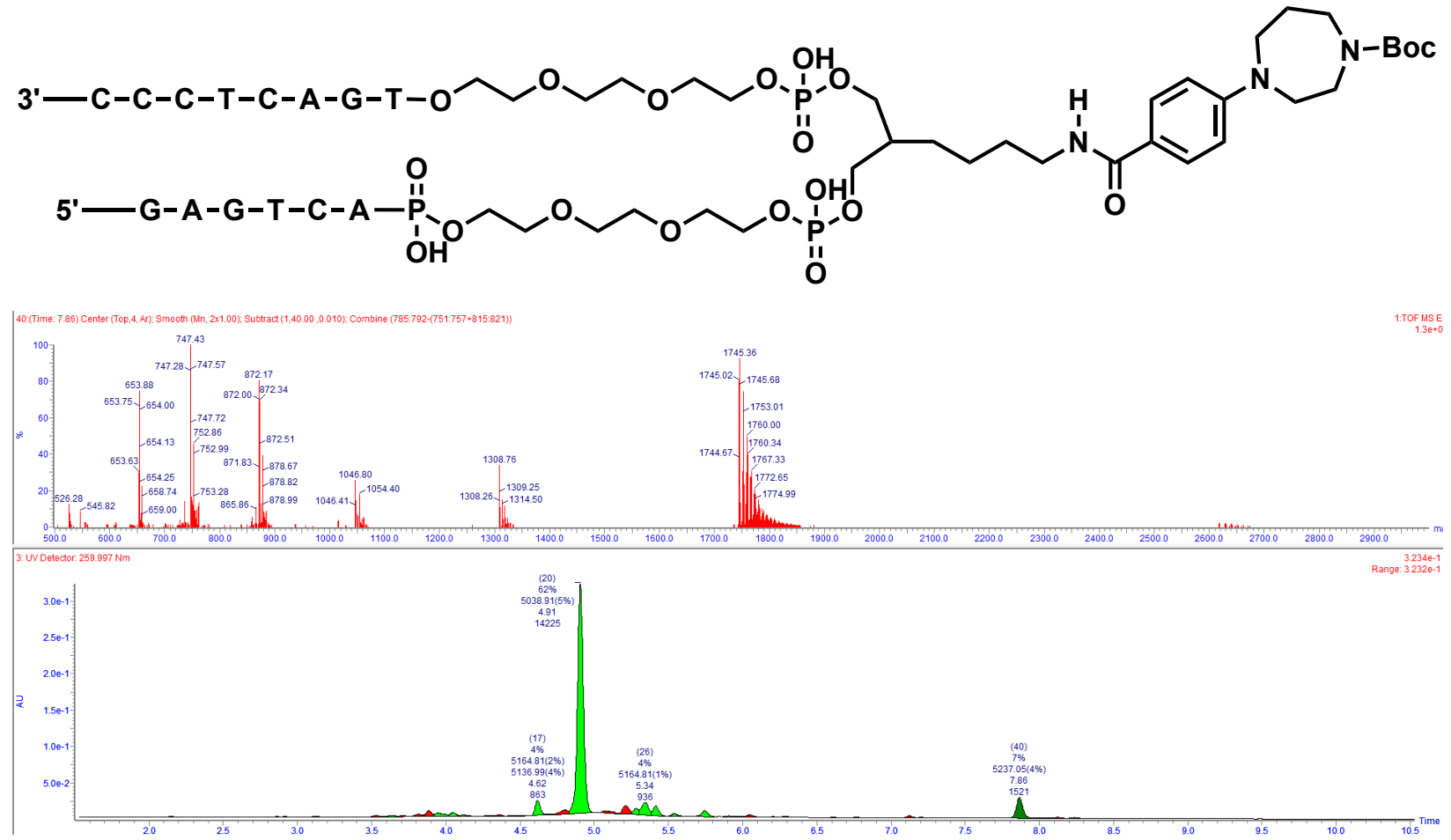


\section{Procedure for Synthesis of AOP-Headpiece 66}

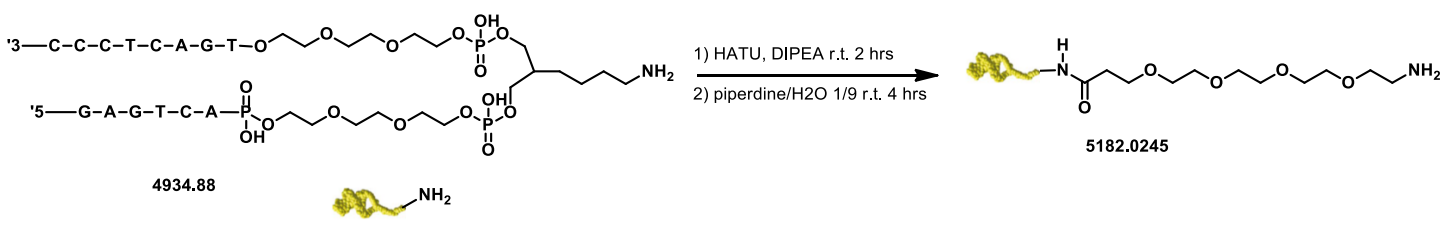

To a $500 \mu \mathrm{L}$ Eppendorf HATU (15.2 mg, $40 \mu \mathrm{mol})$, carboxylic acid S67 (40 $\mu \mathrm{L}, 1.0 \mathrm{M}$ in DMA, $40 \mu \mathrm{mol})$ and DIPEA ( $40 \mu \mathrm{L}, 1.0 \mathrm{M}$ in DMA, $40 \mu \mathrm{mol})$ was added successively. The the resulting mixture was mixed by vortex and left to stand at room temperature for $0.5 \mathrm{~h}$ to give a slightly yellow solution. Next, a stock solution of DNA headpiece $\left(20.0 \mu \mathrm{L}, 20 \mathrm{mM}\right.$ in $\left.\mathrm{H}_{2} \mathrm{O}, 400 \mathrm{nmol}\right)$ was diluted with $100 \mathrm{mM}$ borate buffer $(380 \mu \mathrm{L}, \mathrm{pH} 9.5)$ in a $2.0 \mathrm{~mL}$ Eppendorf tube. Then the previously activated solution (100 equiv) was transferred to the headpiece and briefly mixed by vortex. The resulting reaction mixture was allowed to stand for 2 hours at room temperature. After 2 hours, $5 \mathrm{M} \mathrm{NaCl}(60 \mu \mathrm{L})$ and cold ethanol $(1.2 \mathrm{~mL})$ were added to the reaction mixture sequentially, and the contents were mixed by vortex. The resulting suspension was allowed to stand for 20 minutes at $-20^{\circ} \mathrm{C}$. The suspension was then centrifuged at 10,000 RPM for 2 minutes and the supernatant was discarded. Trace EtOH was removed under vacuum.

The dry DNA pellet was dissolved $(1.6 \mathrm{mM})$ in a mixture of water/piperdine $(250 \mu \mathrm{L}, 9: 1 \mathrm{v} / \mathrm{v})$, a small stir bar was added, and the resulting mixture was stirred at room temperature for 4 hrs. After 4 hours $5 \mathrm{M} \mathrm{NaCl}$ $(60 \mu \mathrm{L})$ After 2 hours, $5 \mathrm{M} \mathrm{NaCl}(60 \mu \mathrm{L})$ and cold ethanol $(1.2 \mathrm{~mL})$ were added to the reaction mixture sequentially, and the contents were mixed by vortex. The resulting suspension was allowed to stand for 20 minutes at $-20^{\circ} \mathrm{C}$. The suspension was then centrifuged at 10,000 RPM for 2 minutes and the supernatant was discarded. Trace EtOH was removed under vacuum.

The pellet obtained was then reconstituted in $\mathrm{H}_{2} \mathrm{O}(80 \mu \mathrm{L})$ to give a stock solution of PEG-DNA at $5 \mathrm{mM}$ concentration. $1 \mu \mathrm{L}$ of the stock solution was transferred to a $500 \mu \mathrm{L}$ Eppendorf, diluted with $99 \mu \mathrm{L}$ of $\mathrm{H} 2 \mathrm{O}$ to make stock solution at $0.1 \mathrm{mM}$ concentration. This dilution process was repeated with $10 \mu \mathrm{L}$ of DNA headpiece $(0.1 \mathrm{mM})$ and $90 \mu \mathrm{L}$ of $\mathrm{H} 2 \mathrm{O}$ to prepare LCMS sample at $0.01 \mathrm{mM}$ concentration. 


\section{Synthesis of DNA-bound Amino Acid SI-12 to SI-13}

The solution of PEG-DNA headpiece $\left(1.0 \mu \mathrm{L}, 20 \mathrm{mM}\right.$ in $\left.\mathrm{H}_{2} \mathrm{O}, 20 \mathrm{nmol}\right)$ was diluted with $100 \mathrm{mM}$ borate buffer $(15 \mu \mathrm{L}, \mathrm{pH} 9.5)$ in a $1.5 \mathrm{~mL}$ eppendorf tube. Then Fmoc Amino acid ( $2 \mu \mathrm{L}, 1 \mathrm{M}$ in DMA, 100 eq.) followed by 4-(4,6-dimethoxy-1,3,5-triazin-2-yl)-4-methylmorpholinium chloride ( $2 \mu \mathrm{L}, 1 \mathrm{M}$ in $\mathrm{H}_{2} \mathrm{O}, 100$ eq.). The contents were mixed by vortex and allowed to stand for 12 hours at room temperature. To the reaction solution was added $5 \mathrm{M} \mathrm{NaCl}(2 \mu \mathrm{L})$ followed by cold ethanol $(1.5 \mathrm{~mL})$ and the contents were mixed by vortex and allowed to stand for 20 minutes at $-20{ }^{\circ} \mathrm{C}$. The suspension was then centrifuged at 10,000 RPM for 5 minutes and the supernatant was discarded and trace of EtOH was removed by vaccum. The above procedure was repeated again because the reaction was not completed. The DNA pellet was dissolved in the mixture of water/piperdine $(50 \mu \mathrm{L}, 9: 1 \mathrm{v} / \mathrm{v})$ and standed at $50{ }^{\circ} \mathrm{C}$ for $4 \mathrm{~h}$. To the reaction solution was added $5 \mathrm{M} \mathrm{NaCl}(5 \mu \mathrm{L})$ followed by cold ethanol $(1.2 \mathrm{~mL})$ and the contents were mixed by vortex and allowed to stand for 20 minutes at $-20^{\circ} \mathrm{C}$. The suspension was then centrifuged at 10,000 RPM for 5 minutes and the supernatant was discarded and trace of EtOH was removed under vaccum.

The pellet obtained was reconstituted in $\mathrm{H}_{2} \mathrm{O}(200 \mu \mathrm{L})$ to give a stock solution of DNA-bound amino acid at $0.1 \mathrm{mM}$ concentration. $50 \mu \mathrm{L}$ of the solution was transferred to $300 \mu \mathrm{L}$ vial for LCMS. 


\section{LC Trace and Mass of SI-12}

With 66

Exact mass: 5315.08

Triply charged mass [M-3]/3, caluculated: 1770.69 ; observed 1771.36
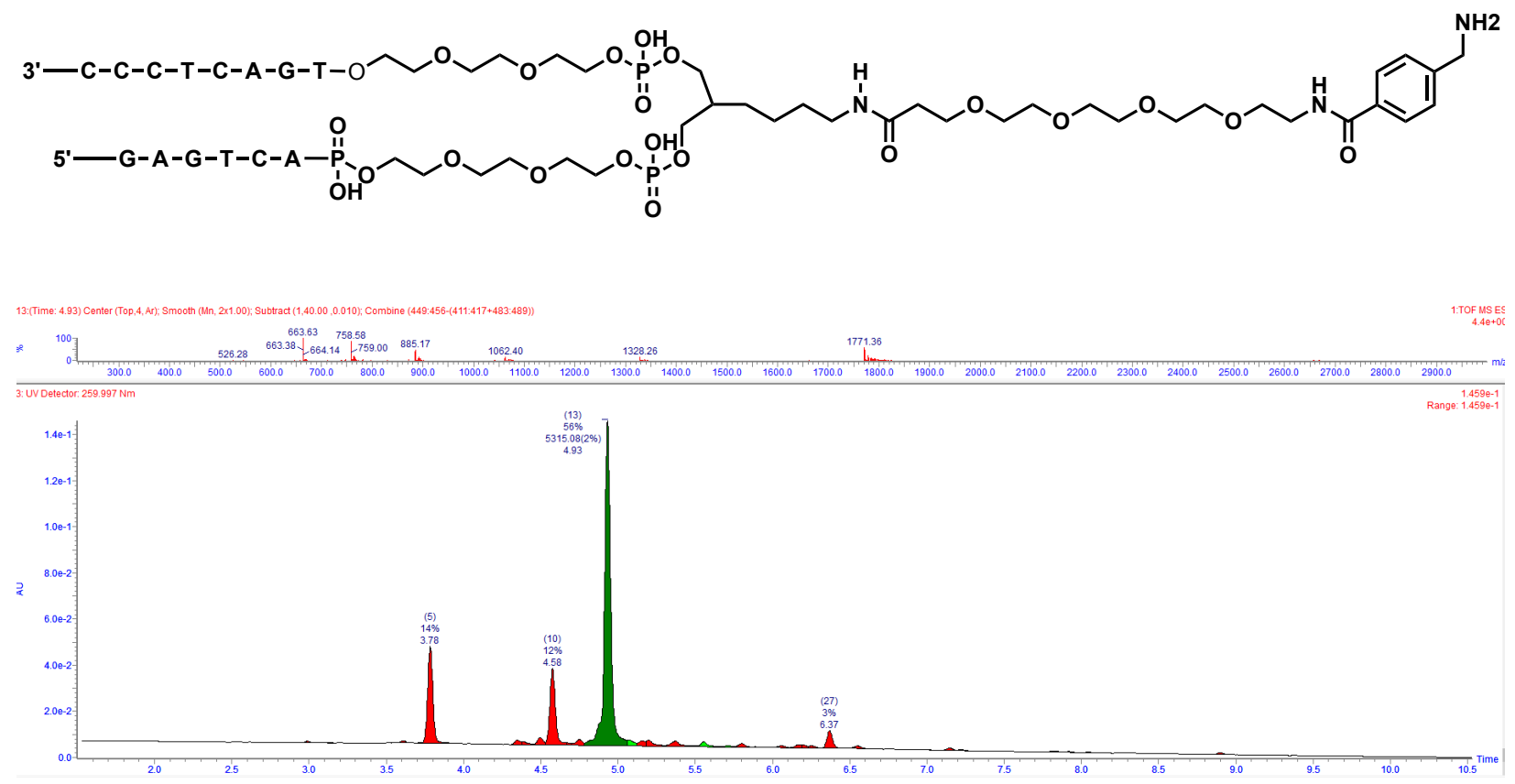


\section{LC Trace and Mass of SI-13}

With 66

Exact mass: 5265.06

Triply charged mass [M-3]/3, caluculated: 1754.02; observed 1754.02
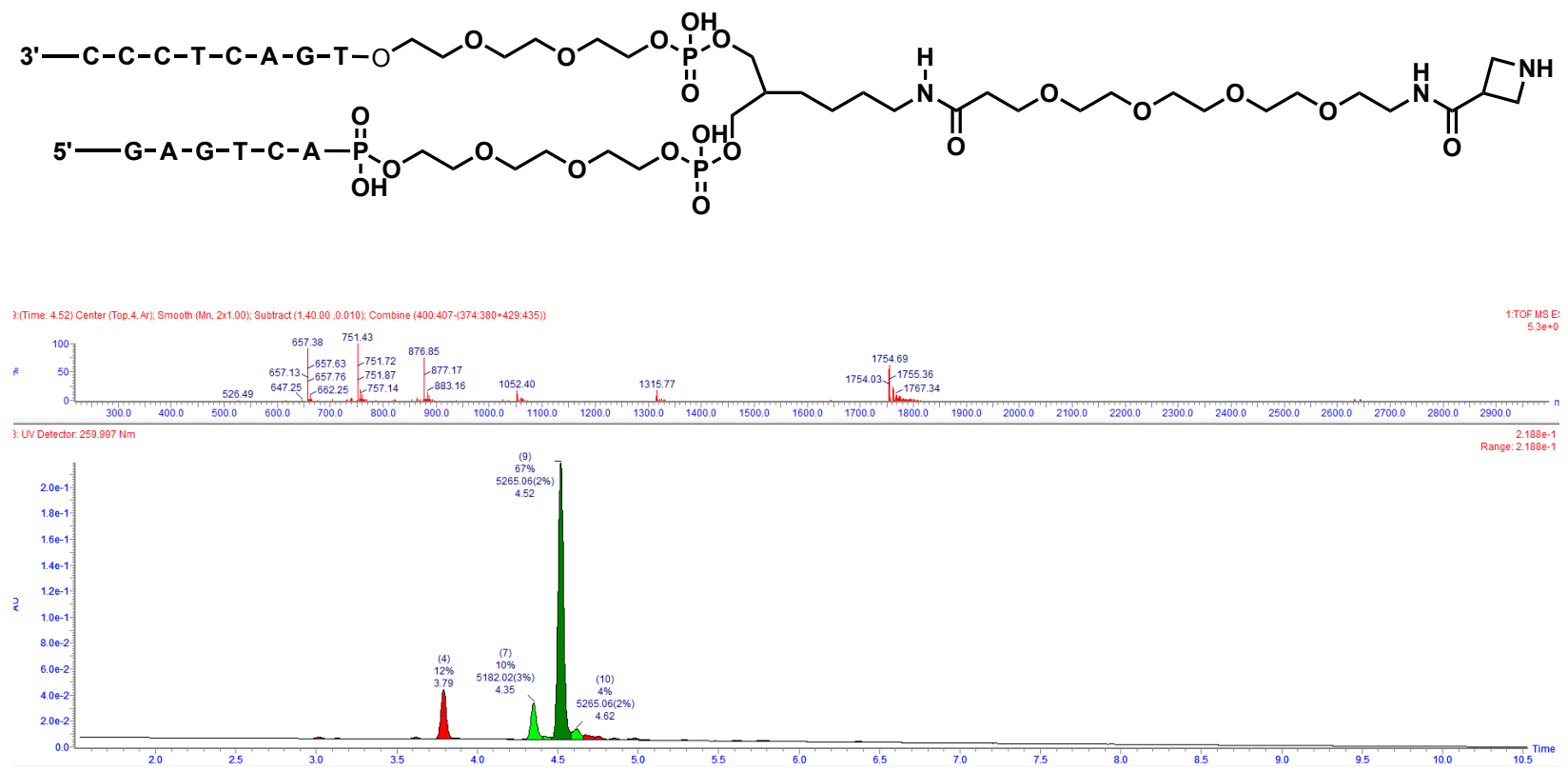


\section{General procedure for reductive amination}

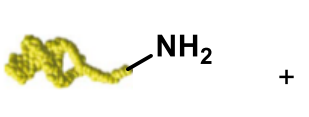

PEG-DNA 5182.0245

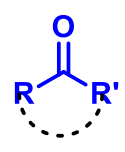

$500 \mathrm{mM}$

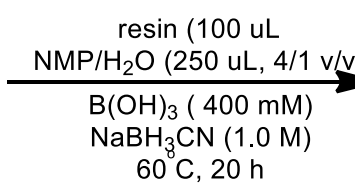

$60^{\circ} \mathrm{C}, 20 \mathrm{~h}$

Resin slurry (100 $\mu \mathrm{L}$, in 1:1 PBS:ACN) and PBS buffer $(500 \mu \mathrm{L})$ were added to a $2 \mathrm{~mL}$ Eppendorf tube and vortexed. The tube was then centrifuged and the supernatant discard. Next, a stock solution of DNA headpiece $(2.0 \mu \mathrm{L}, 5 \mathrm{mM}$ in $\mathrm{H} 2 \mathrm{O}, 10 \mathrm{nmol})$ was diluted with PBS buffer $(100 \mu \mathrm{L})$ and added to the resin bed The mixture was vortexed and allowed to shake for $15 \mathrm{~min}$ to load the DNA. The resin mixture was centrifuged and the supernatant discarded. The result resin bed was then washed with NMP $(2 * 500 \mu \mathrm{L})$. To the resin mixture, NMP $(200 \mu \mathrm{L}), \mathrm{B}(\mathrm{OH})_{3}(9 \mathrm{mg}, 400 \mathrm{mM})$, 4-heptanone $(18 \mu \mathrm{L}, 500 \mathrm{mM})$, a small stirbar, $\mathrm{NaBH}_{3} \mathrm{CN}(16 \mathrm{mg}$ as solid, $1 \mathrm{M})$ and water $(50 \mu \mathrm{L})$ were added sequentially. The reaction mixture was stirred for $20 \mathrm{hrs}$ at $60^{\circ} \mathrm{C}$. After 20 hours NMP $(250 \mu \mathrm{L})$ was added to the reaction mixture, votexed, centrifuged and the supernatant discarded. The reaction was eluted and EtOH precipitated by Protocol XX. The resultant DNA pellet was dissolved in $100 \mu \mathrm{L}$ of $\mathrm{H}_{2} \mathrm{O}$, and analyzed by HPLS-MS. When Calculating yields, the peak at $\sim 3.78$ minutes (mass: 4976 ) was disregarded and not used in the yield calculation. This peak was an acylated DNA headpiece that resulted during synthesis of AOP headpiece 66. 


\section{Protocol 7: On-DNA Reductive Amination}

1. Prepare DNA and resin as described in Protocol 1.

2. Wash resin bed with NMP $(2 \times 0.5 \mathrm{~mL})$ and a small stir bar was placed in the tube.

3. Weight out $\mathrm{B}(\mathrm{OH})_{3}(9 \mathrm{mg}, 400 \mathrm{mM}), \mathrm{NaBH}_{3} \mathrm{CN}(16 \mathrm{mg}, 1 \mathrm{M})$, and ketone/aldehyde (if solid) $(500 \mathrm{mM})$ in separate Eppendorf tubes.

4. Dissolve $\mathrm{B}(\mathrm{OH})_{3}$ in $200 \mu \mathrm{L} \mathrm{NMP}$ and use this solution to dissolve the ketone.

5. This solution was transferred to the resin bed and solid $\mathrm{NaBH}_{3} \mathrm{CN}$ was dumped in, and $50 \mu \mathrm{L}$ water was added sequentially.

6. The reaction tube was capped and placed in heating block at $60^{\circ} \mathrm{C}$ and allowed to stir for $18-24$ hours.

7. After the reaction, the resin was washed, eluted, and DNA characterized as described above (Protocol 2 and 3). 
Graphical Supporting Information: On-DNA Reductive Amination

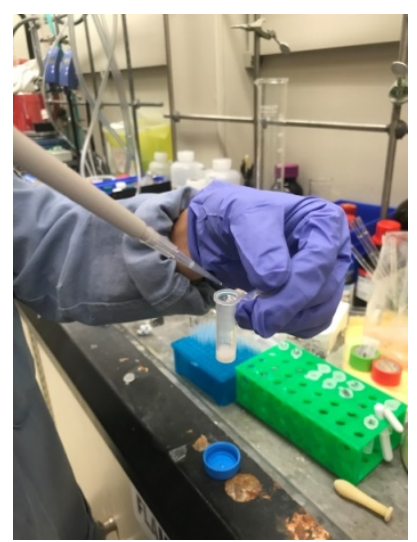

Ketone or aldehyde added to tube with $200 \mu \mathrm{L}$ NMO

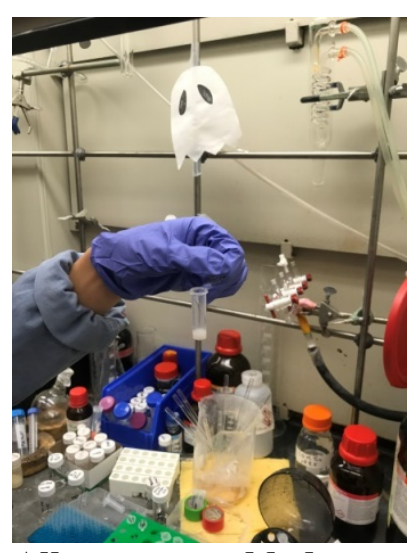

All contents added to reaction tube

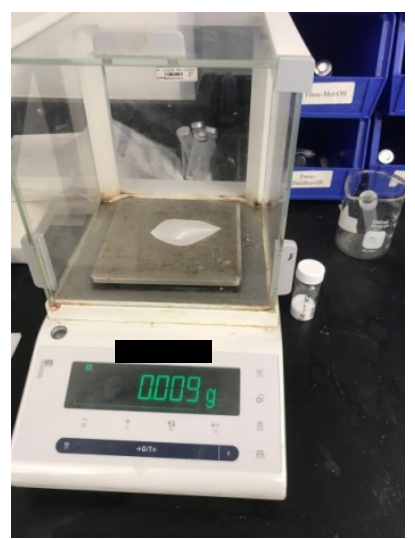

Boric Acid was Weighed out

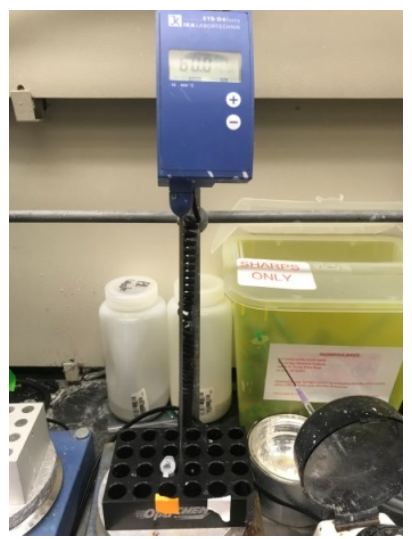

Reaction running at $60^{\circ} \mathrm{C}$ for 20 hours

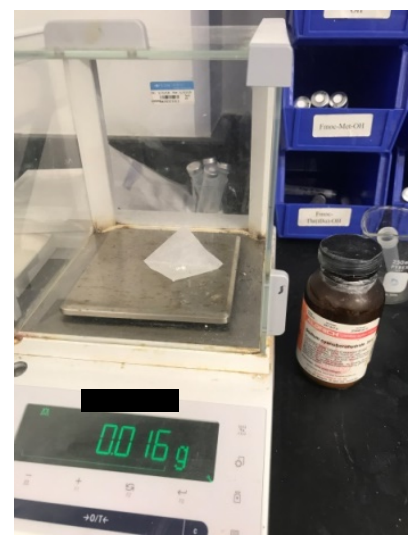

Sodium Cyanoborohydride was weighed

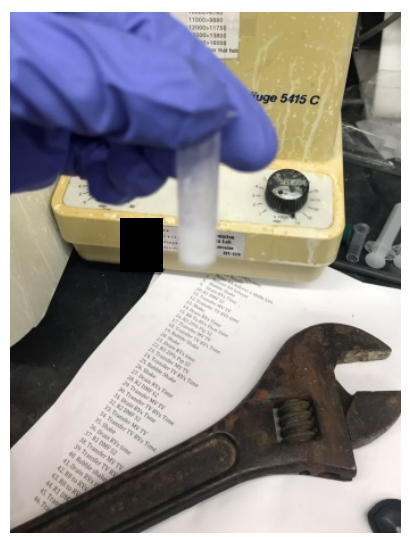

Reaction after 20 hours 


\section{Trouble Shooting: Frequently Asked Questions (On-DNA Reductive Amination Reaction)}

Should I stir or shake this reaction?

We found that stirring the reaction usually resulted in higher yield than a shaken reaction. Upon addition, the $\mathrm{NaBH}_{3} \mathrm{CN}$, does not fully dissolve, thus the amount of the reductant in solution may be influenced by stirring.

How should I add the $\mathrm{B}(\mathrm{OH})_{3}$ and $\mathrm{NaBH}_{3} \mathrm{CN}$ ?

These two compounds were usually added (dumped in) as solid. The Boric acid can be used as a stock solution.

After centrifuging the finished reaction, the reaction mixture still looks muddy (turbid), how should I remove the supernatant without removing the resin?

After the reaction was done, NMP $(500 \mu \mathrm{L})$ was added and then centrifuged for 2 minutes. The supernatant was still muddy, but you could see clearly that the resin was in the bottom of Eppendorf. Be careful to remove the supernatant and you may leave a little supernatant behind and wash again to avoid removing the resin. 


\section{Characterization of on-DNA Reductive Amination Reaction Products}

\section{LC Trace and Mass of 68}

Following General Procedure 7 with 66

Yield: $77 \%$

Exact mass: 5280.13

Triply charged mass [M-3]/3, caluculated: 1759.04; observed 1759.04
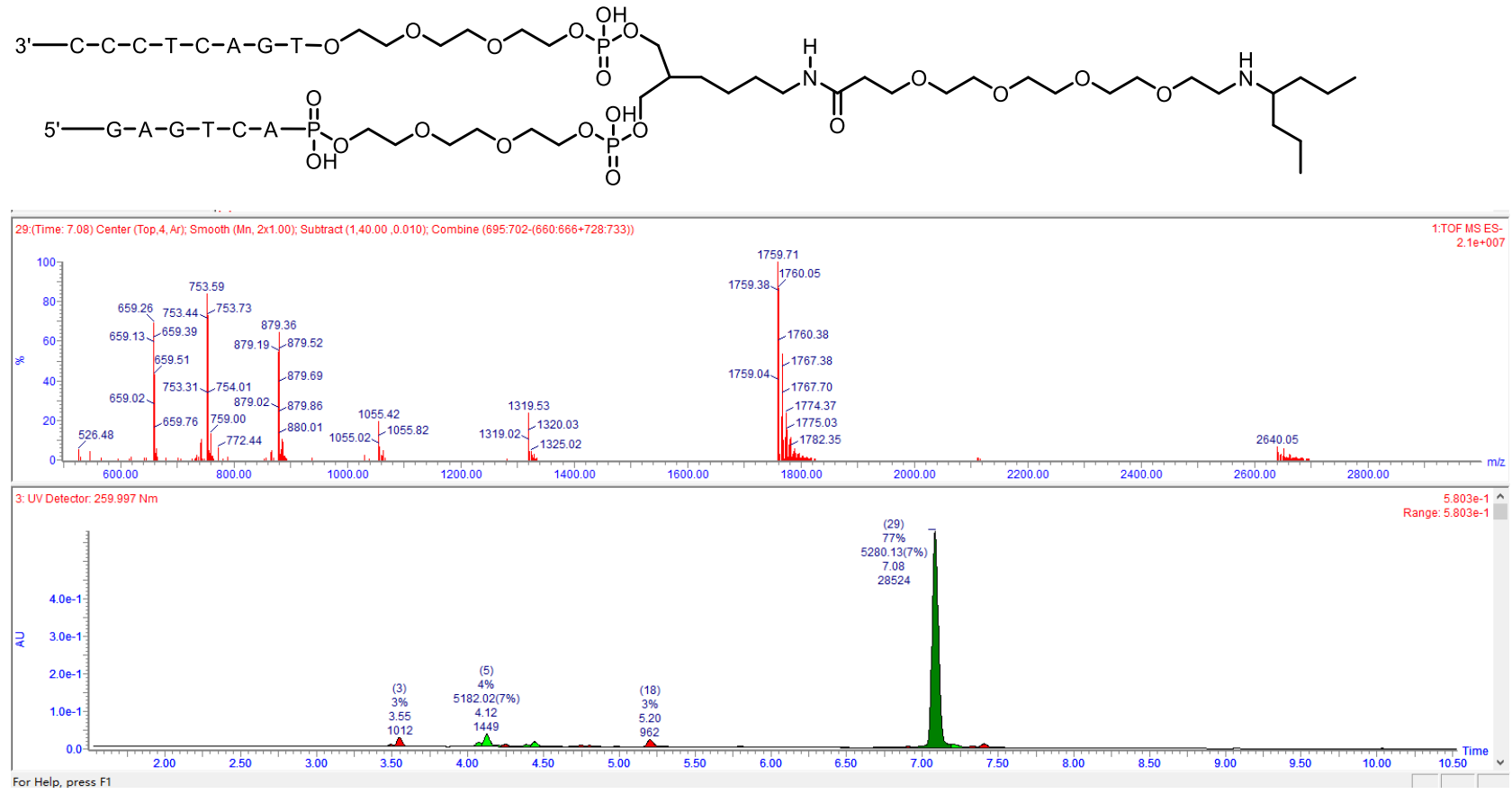


\section{LC Trace and Mass of 69 in tripliacate}

Following General Procedure 7 with 66

Yields: $84,78,84 \%$

Average Yield: 82\%

SDs: 3.4

Exact masses: 5224.07

Triply charged masses [M-3]/3, caluculated: 1740.35; observed 1740.35, 1740.35, 1740.35
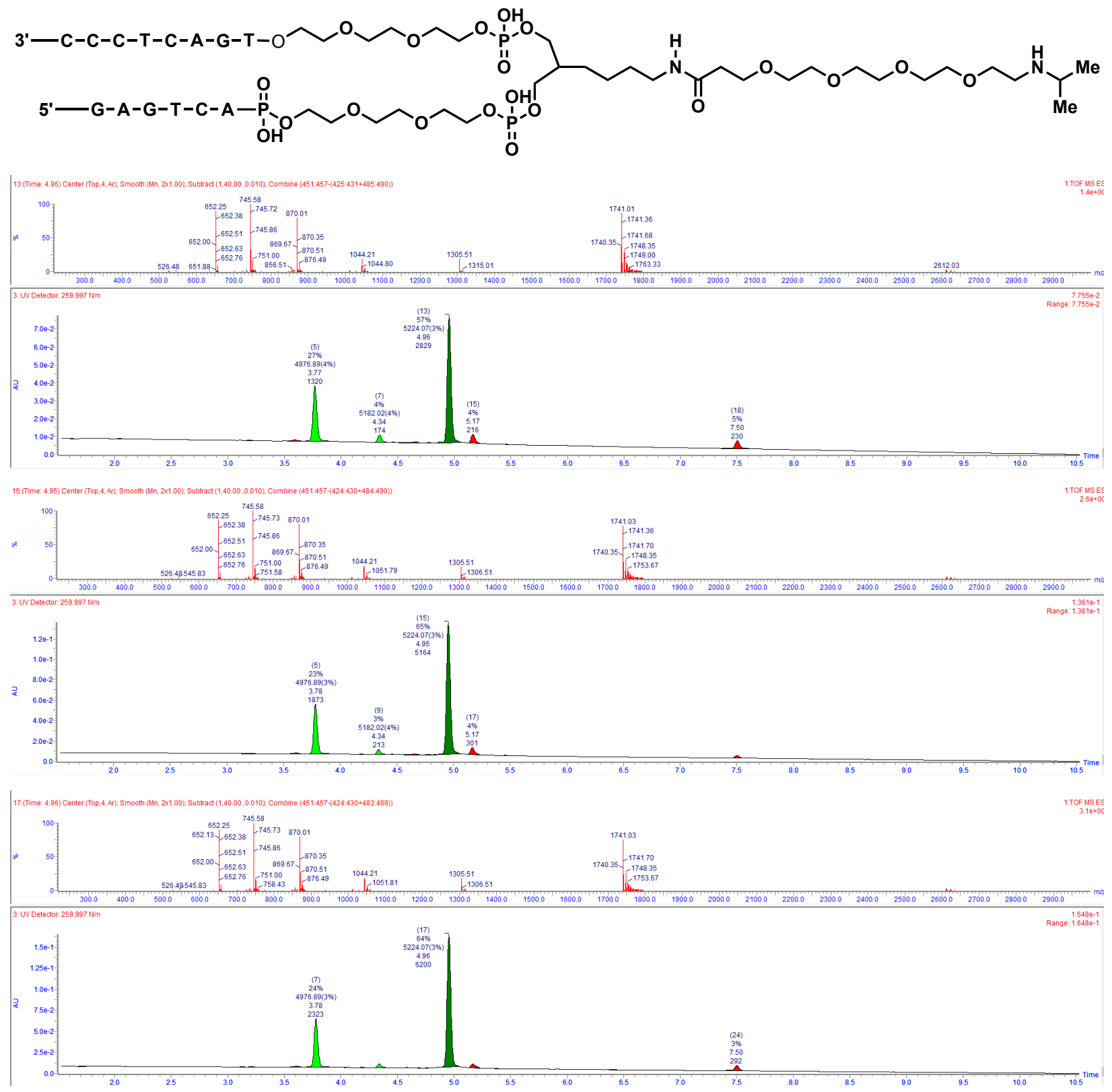


\section{LC Trace and Mass of 69 (Aqueous Reaction)}

Following General Procedure 7 with 66

Yields: $85 \%$

Exact masses: 5224.07

Triply charged masses [M-3]/3, caluculated: 1740.35; observed 1740.35
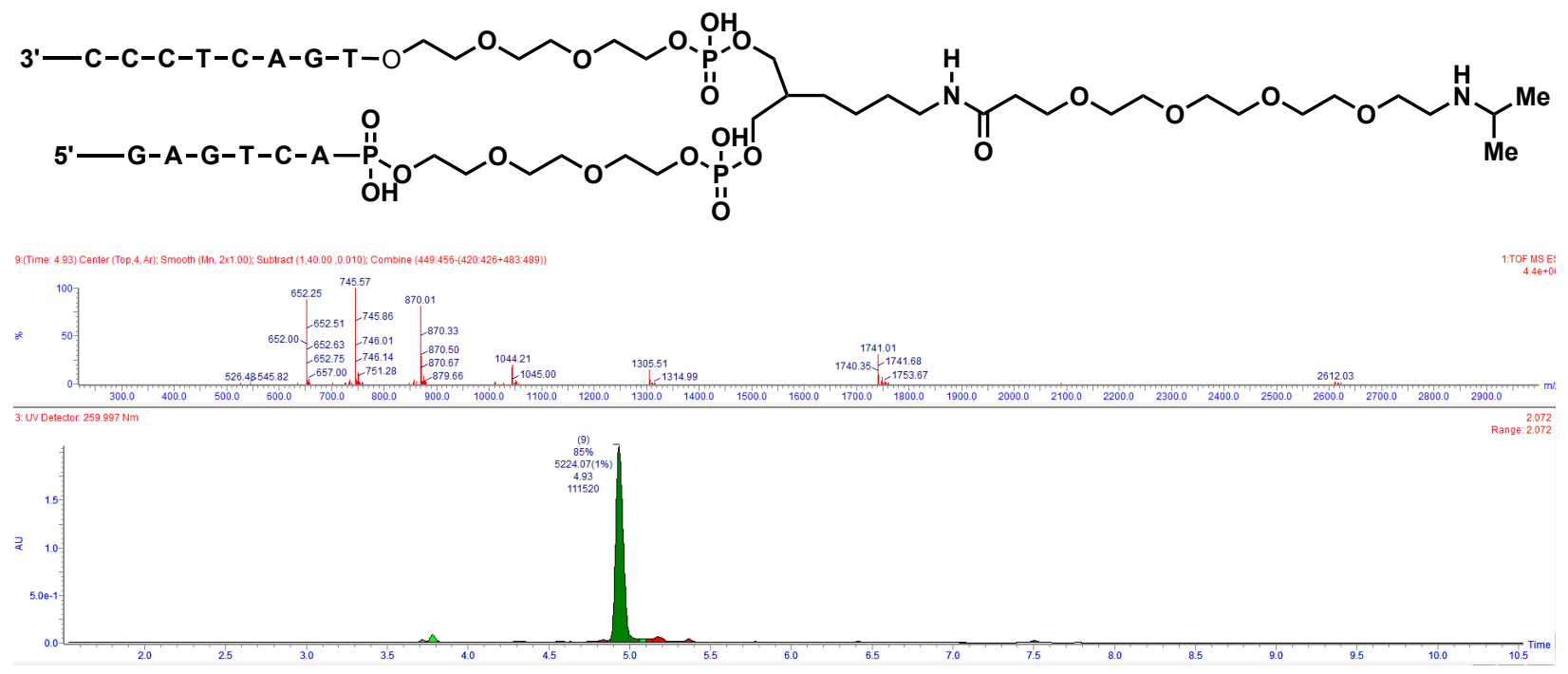


\section{LC Trace and Mass of 70 in tripliacate}

Following General Procedure 7 with 66

Yields: $62,72,70 \%$

Average Yield: 68\%

SDs: 5.3

Exact masses: 5316.18

Triply charged masses [M-3]/3, caluculated: 1771.06; observed 1771.03, 1771.03, 1771.03
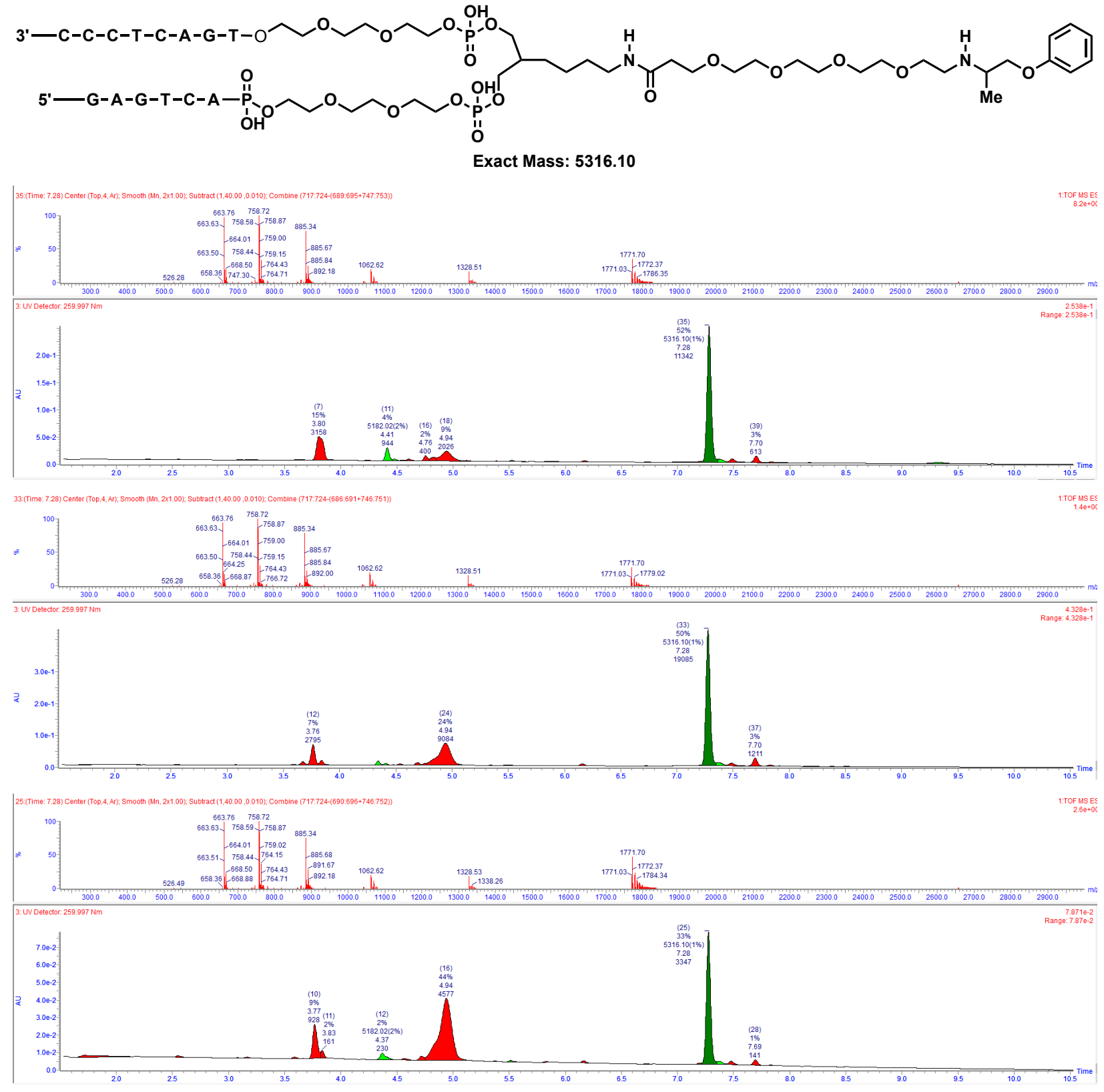


\section{LC Trace and Mass of 70 (Aqueous Reaction)}

Following General Procedure 7 with 66

Yields: $41 \%$

Exact masses: 5316.18

Triply charged masses [M-3]/3, caluculated: 1771.06; observed 1771.7
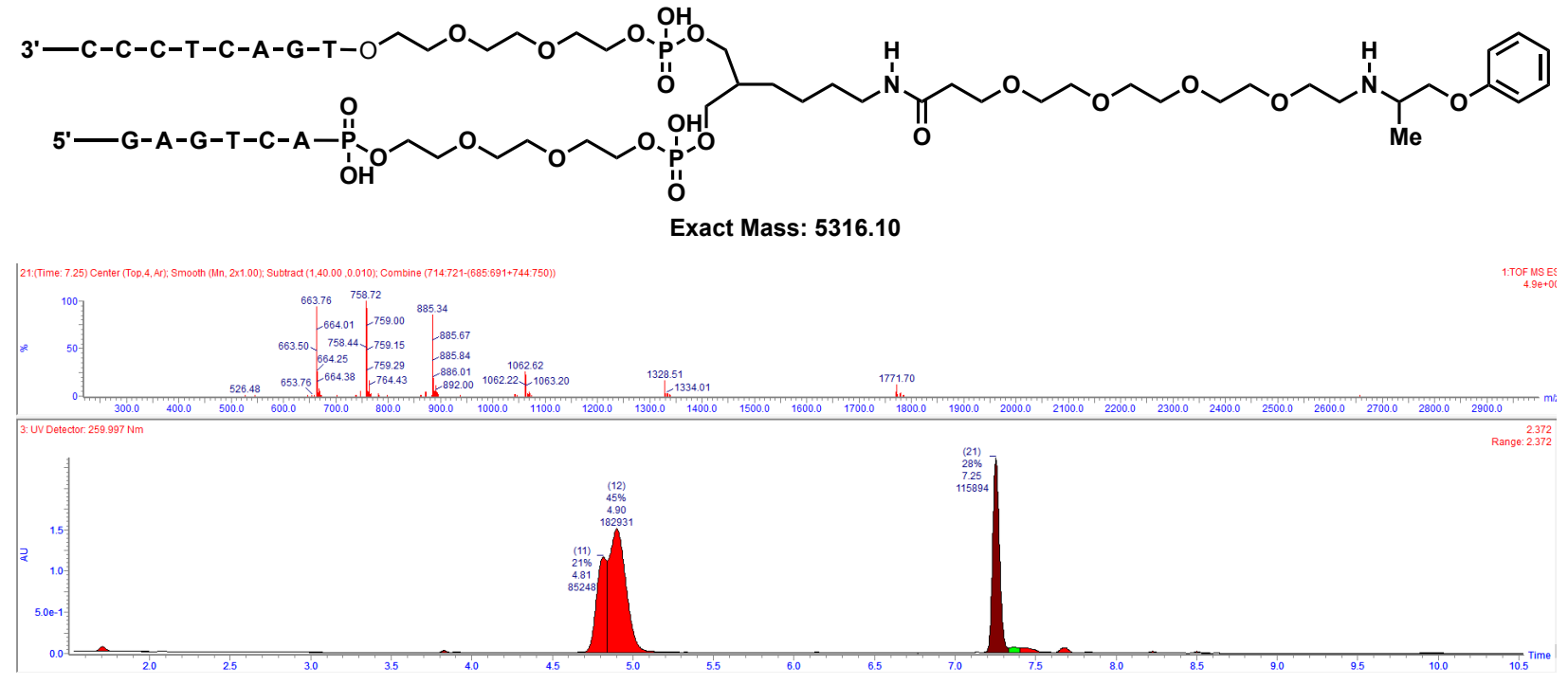


\section{LC Trace and Mass of 71 in tripliacate}

Following General Procedure 7 with 66

Yields: 71, 75, 73\%

Average Yield: $73 \%$

SDs: 2

Exact masses: 5250.09

Triply charged masses [M-3]/3, caluculated: 1749.03 ; observed 1749.02, 1749.02, 1749.02
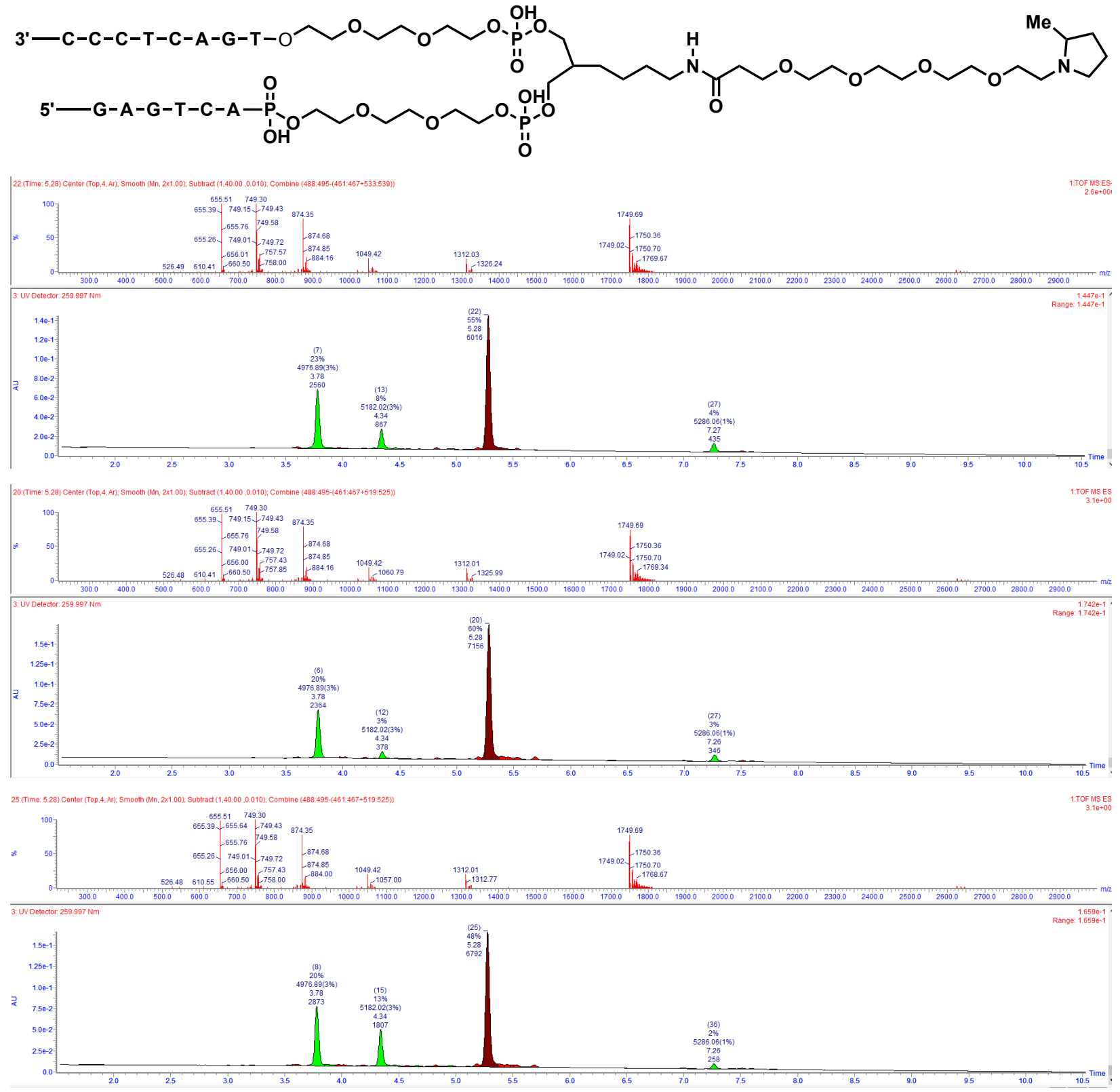


\section{LC Trace and Mass of 71 (Aqueous Reaction)}

Following General Procedure 7 with 66

Yields: $52 \%$

Exact masses: 5250.09

Triply charged masses [M-3]/3, caluculated: 1749.03 ; observed 1749.02
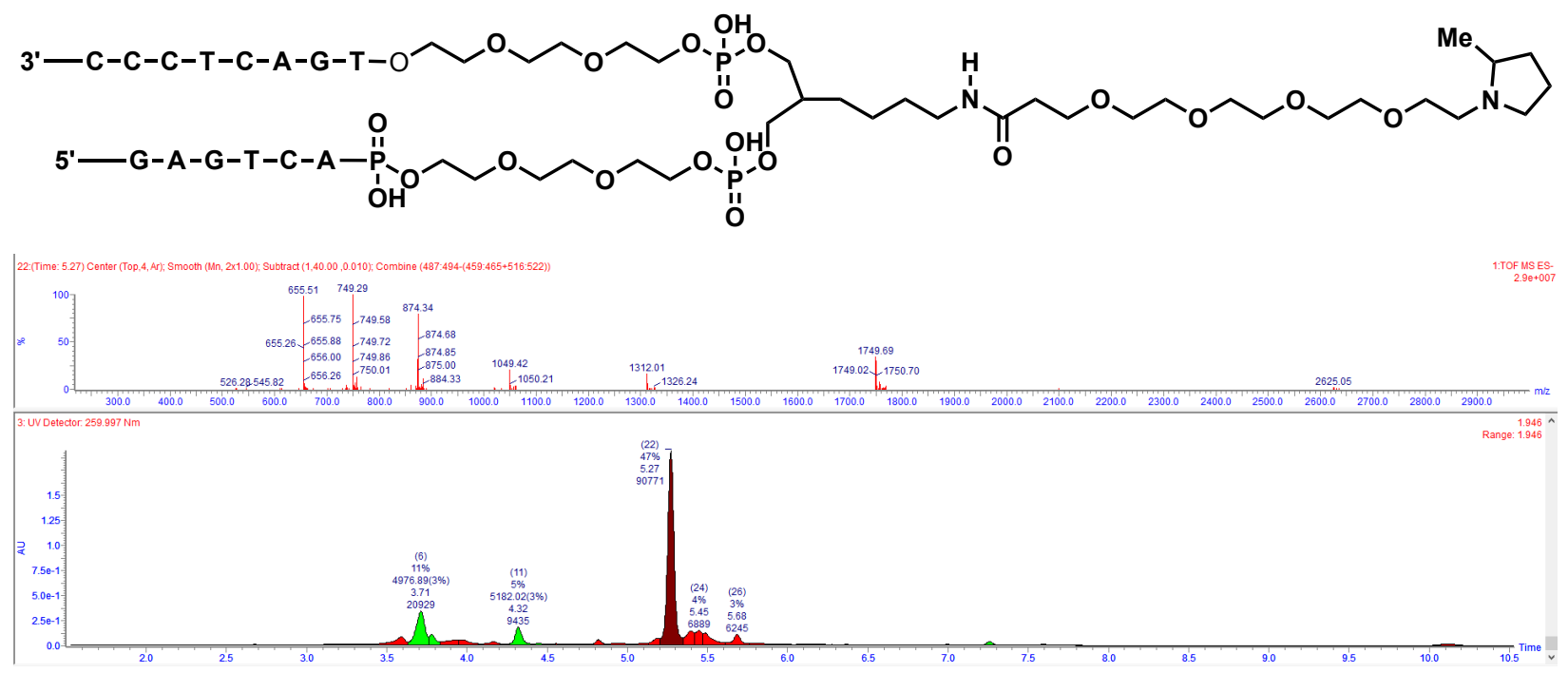


\section{LC Trace and Mass of 72 in tripliacate}

Following General Procedure 7 with 66

Yields: $15,16,18 \%$

Average Yield: 16.3\%

SDs: 1.5

Exact masses: 5250.09

Triply charged masses [M-3]/3, caluculated: 1749.03; observed 1749.02, 1749.02, 1749.02
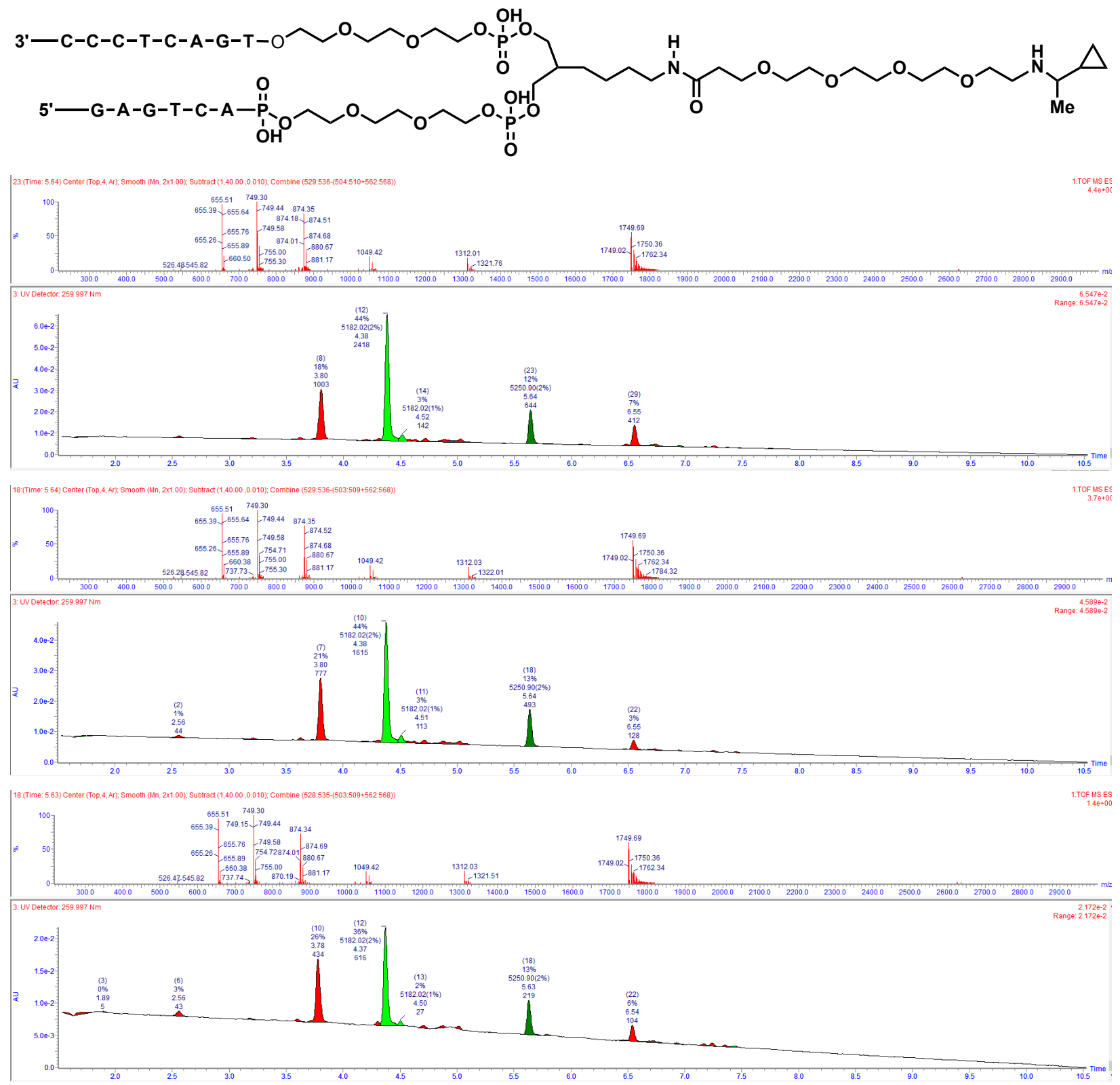


\section{LC Trace and Mass of 72 (Aqueous Reaction)}

Following General Procedure 7 with 66

Yields: $75 \%$

Exact masses: 5250.09

Triply charged masses [M-3]/3, caluculated: 1749.03; observed 1749.71,
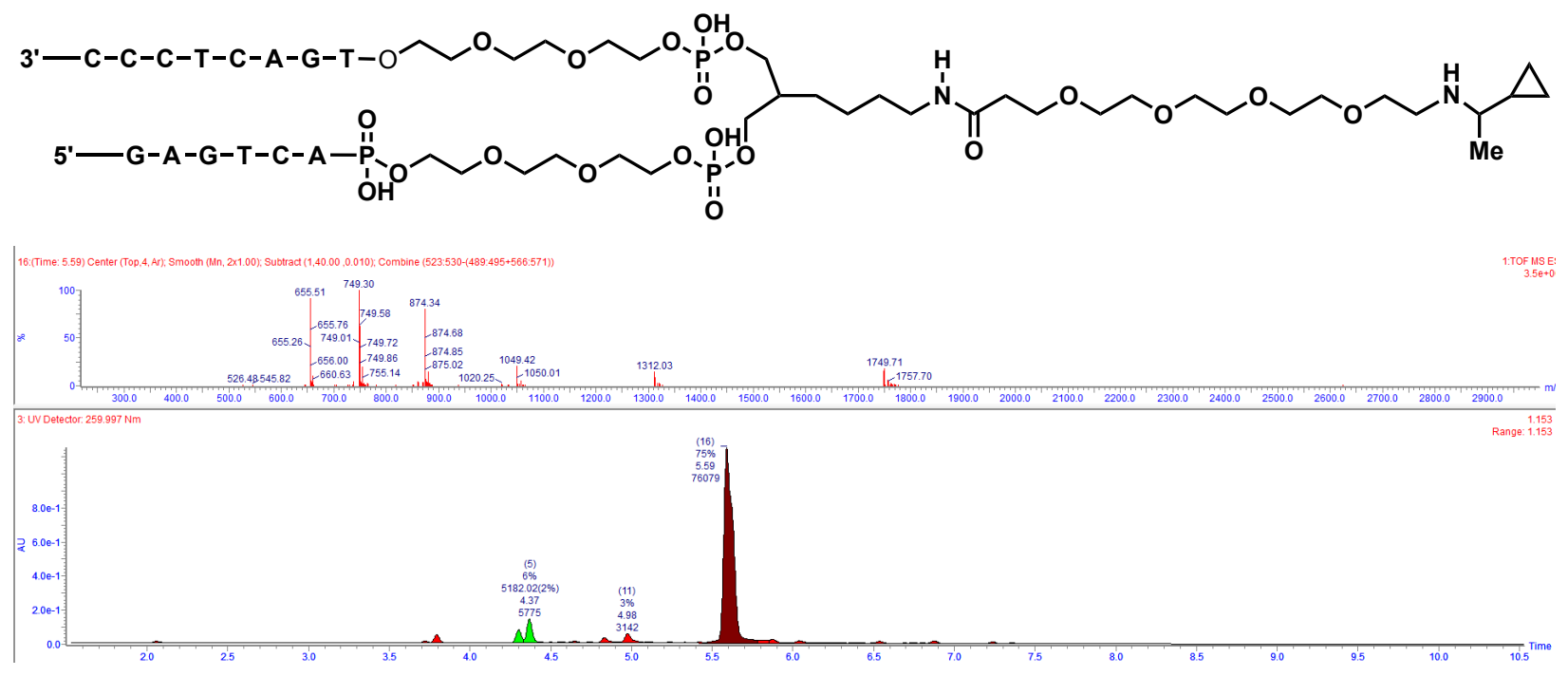


\section{LC Trace and Mass of 73 in tripliacate}

Following General Procedure 7 with 66

Yields: 77, 78, 82\%

Average Yield: $79 \%$

SDs: 2.6

Exact masses: 5266.08

Triply charged masses [M-3]/3, caluculated: 1754.36; observed 1753.68, 1753.68, 1753.68,
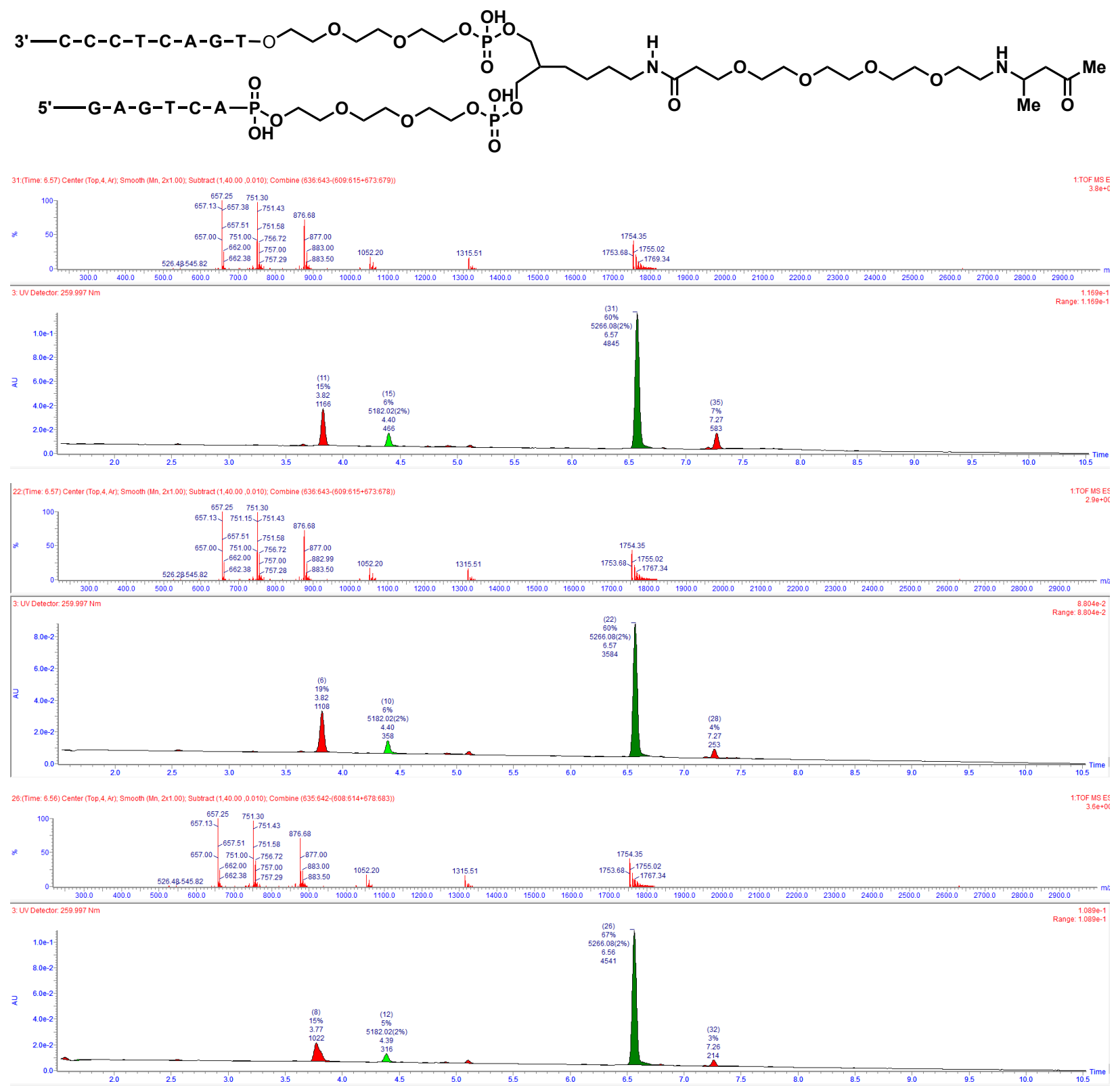


\section{LC Trace and Mass of 73 (Aqueous Reaction)}

Following General Procedure 7 with 66

Yields: $70 \%$

Exact masses: 5250.09

Triply charged masses [M-3]/3, caluculated: 1754.36; observed 1754.35,
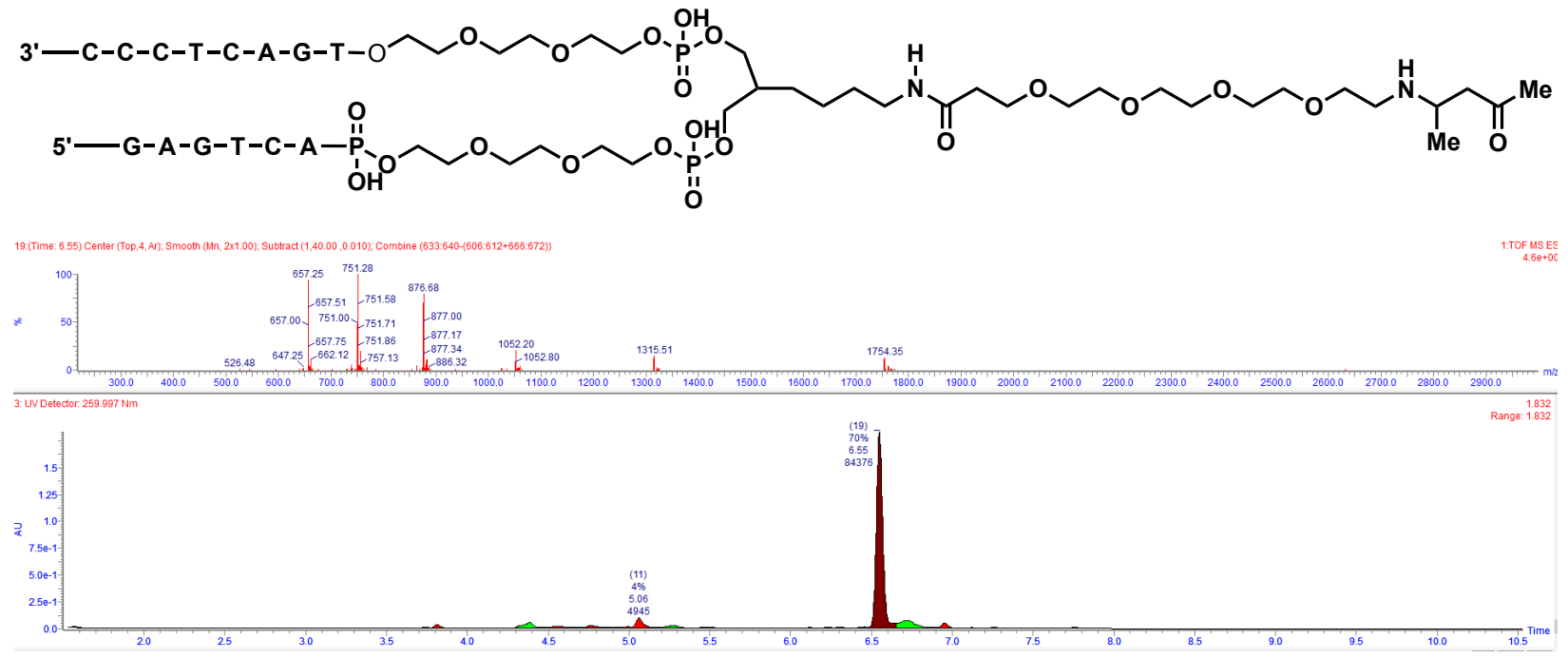


\section{LC Trace and Mass of 74 in tripliacate}

Following General Procedure 7 with 66

Yields: 69, 67, 71\%

Average Yield: 69\%

SDs: 2

Exact masses: 5440.02

Triply charged masses [M-3]/3, caluculated: 1812.34; observed 1749.02, 1749.02, 1749.02
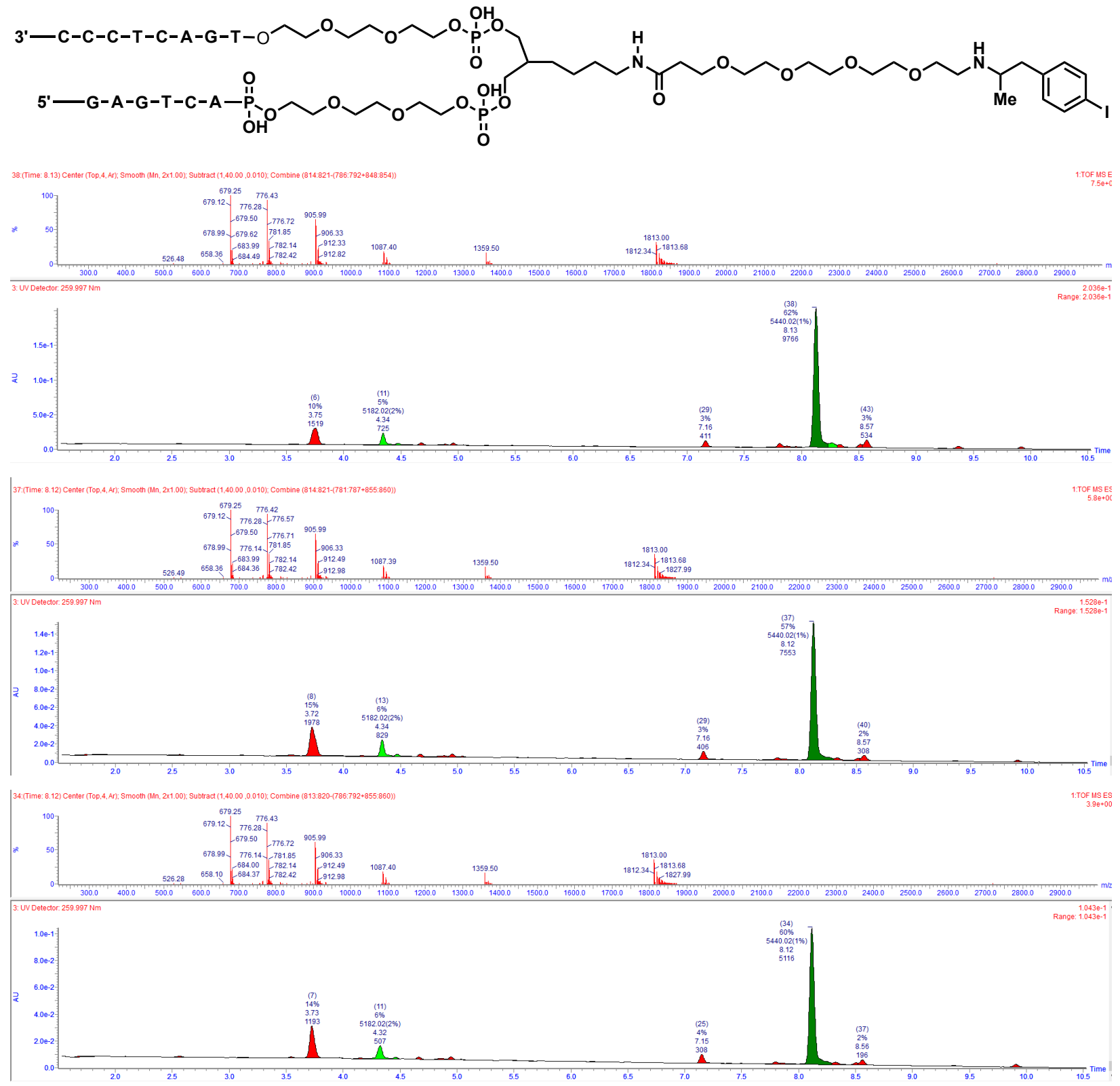


\section{LC Trace and Mass of 74 (Aqueous Reaction)}

Following General Procedure 7 with 66

Yields: $81 \%$

Exact masses: 5440.02

Triply charged masses [M-3]/3, caluculated: 1812.34; observed 1749.71,
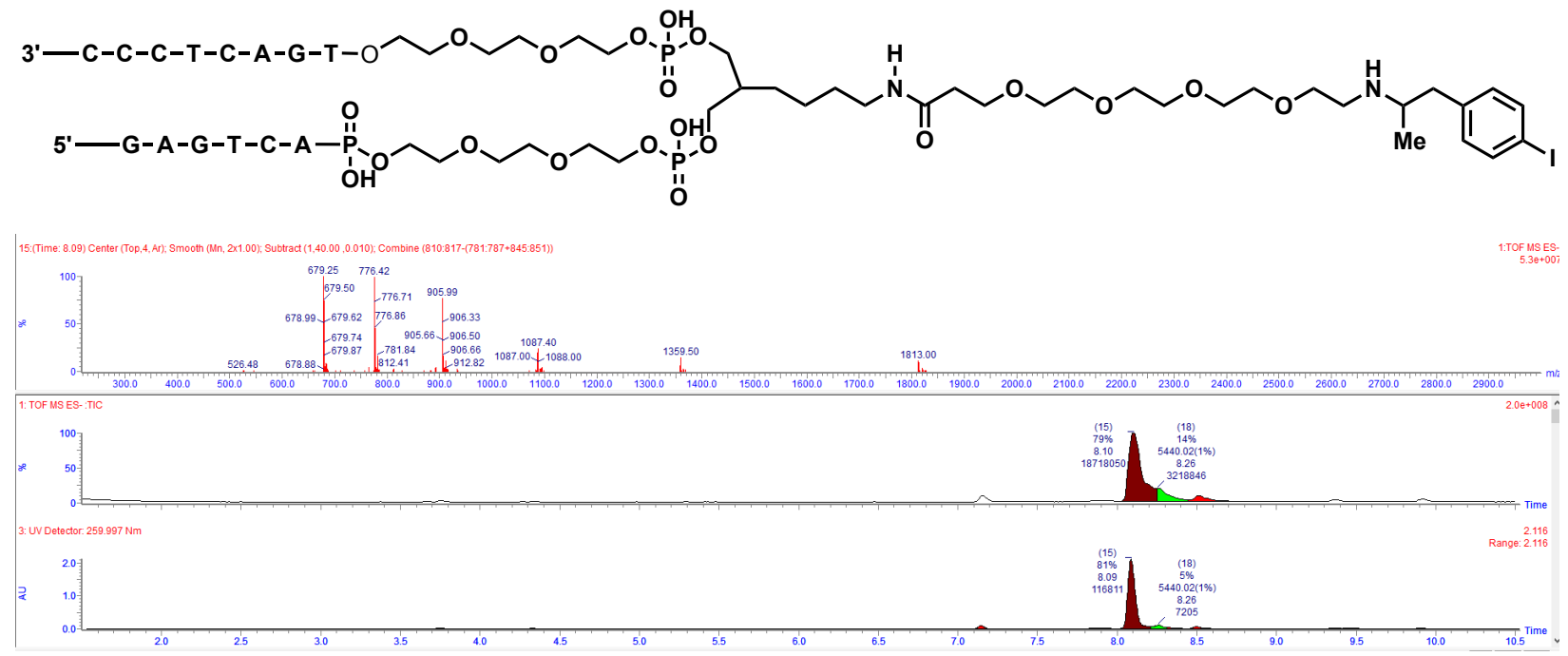


\section{LC Trace and Mass of 75 in tripliacate}

Following General Procedure 7 with 66

Yields: 71, 76, 68\%

Average Yield: $72.66 \%$

SDs: 4.2

Exact masses: 5250.09

Triply charged masses [M-3]/3, caluculated: 1749.03 ; observed 1749.02, 1749.02, 1749.02
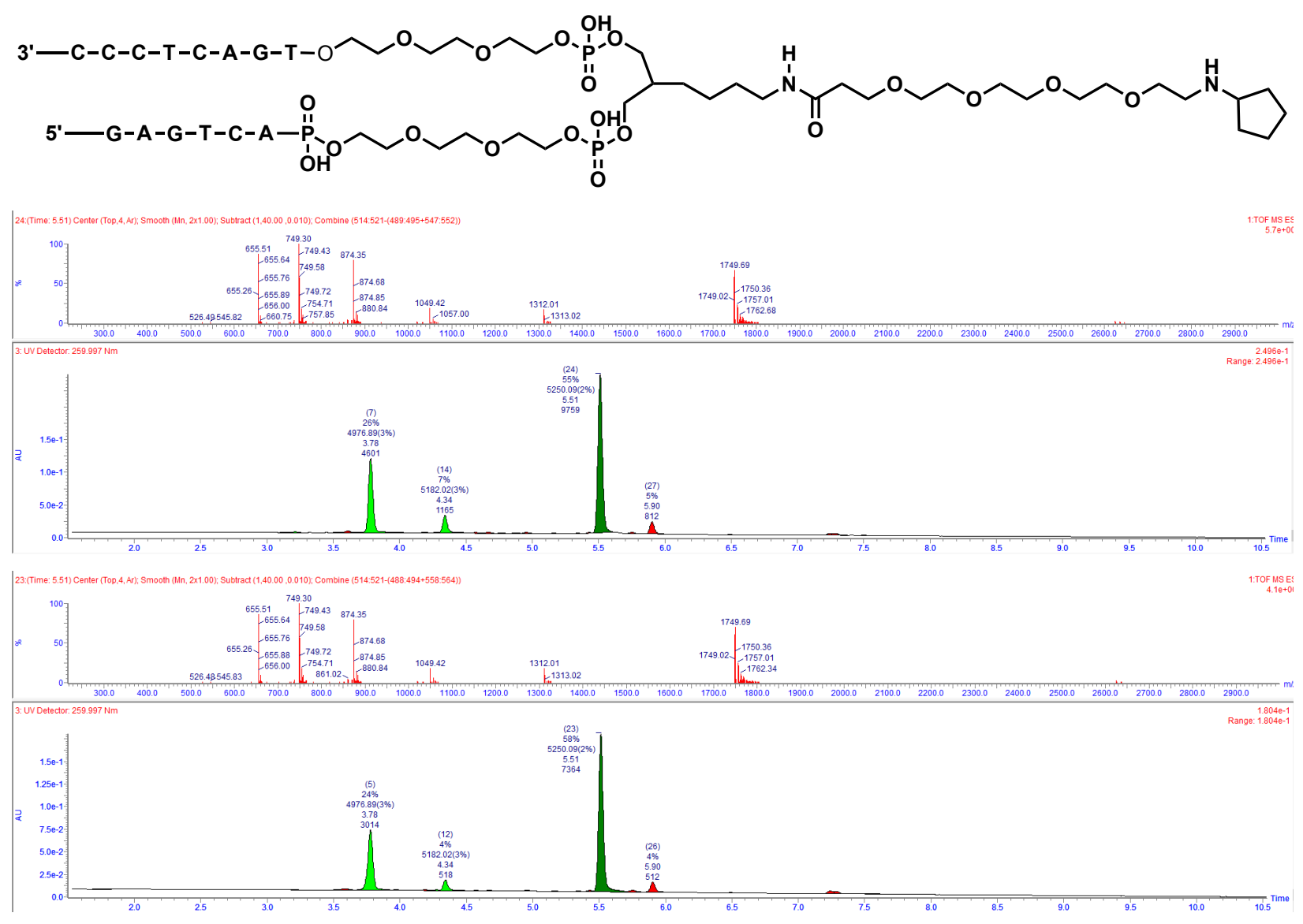

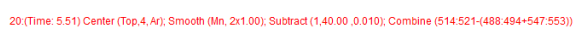
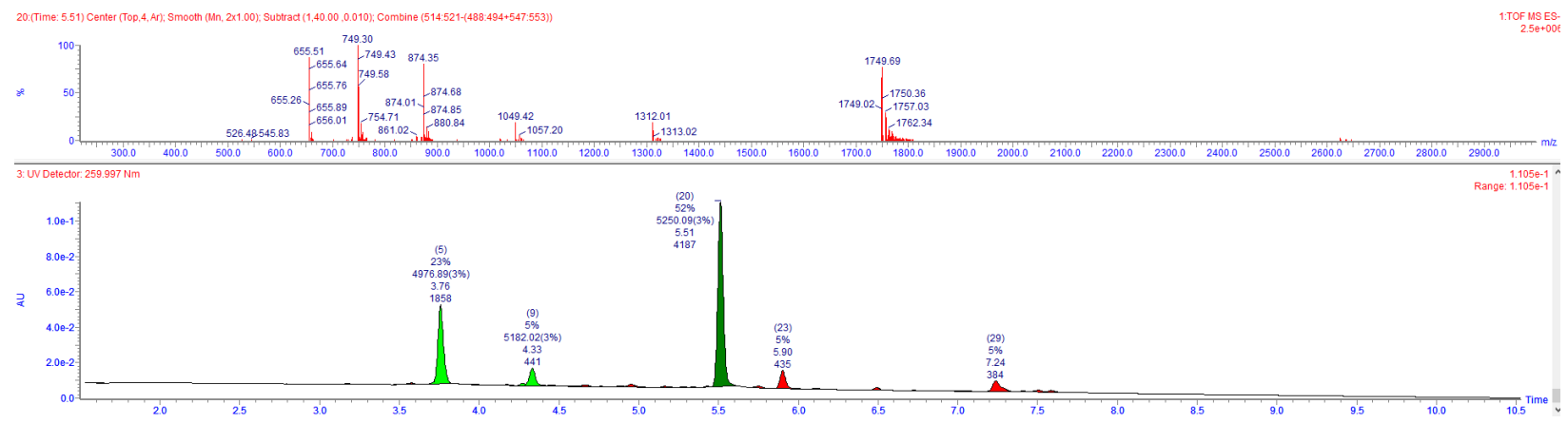


\section{LC Trace and Mass of 75 (Aqueous Reaction)}

Following General Procedure 7 with 66

Yields: $84 \%$

Exact masses: 5250.09

Triply charged masses [M-3]/3, caluculated: 1749.03 ; observed 1749.02
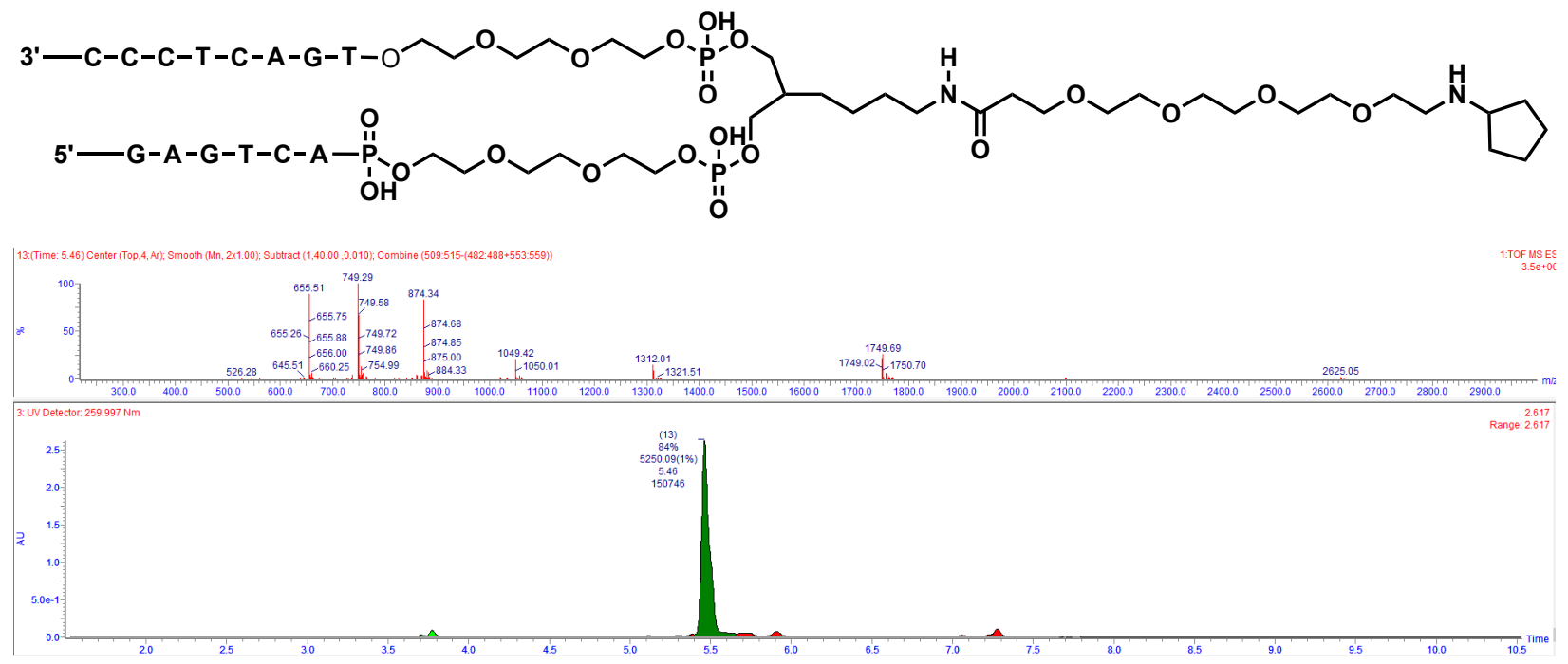


\section{LC Trace and Mass of 76 in tripliacate}

Following General Procedure 7 with 66

Yields: 76, 82, 71\%

Average Yield: 68\%

SDs: 5.5

Exact masses: 5264.10

Triply charged masses [M-3]/3, caluculated: 1753.7; observed 1753.7 , $1753.7,1753.7$
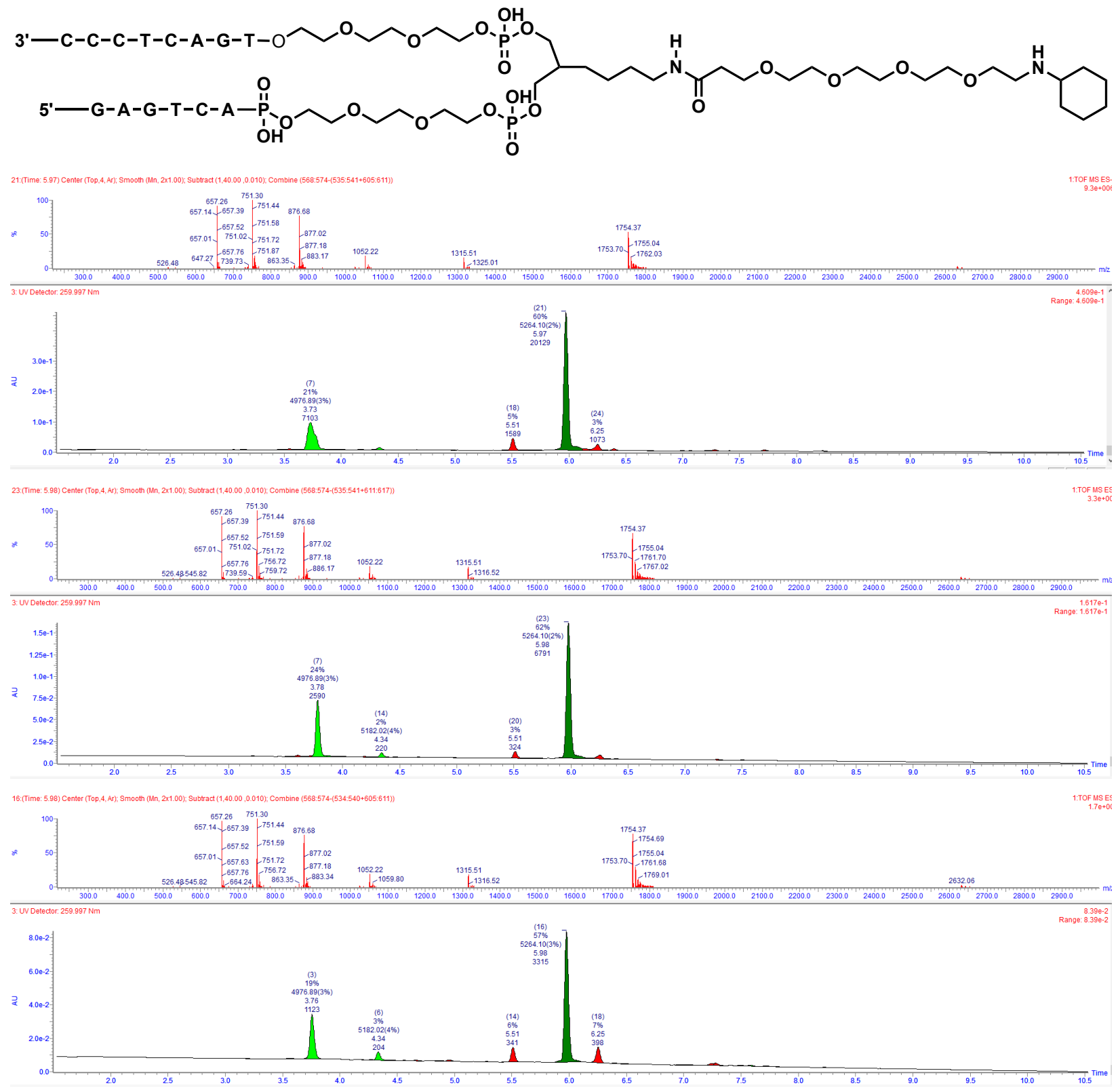


\section{LC Trace and Mass of 76 (Aqueous Reaction)}

Following General Procedure XX with 66

Yields: $68 \%$

Exact masses: 5264.10

Triply charged masses [M-3]/3, caluculated: 1753.7; observed 1753.7
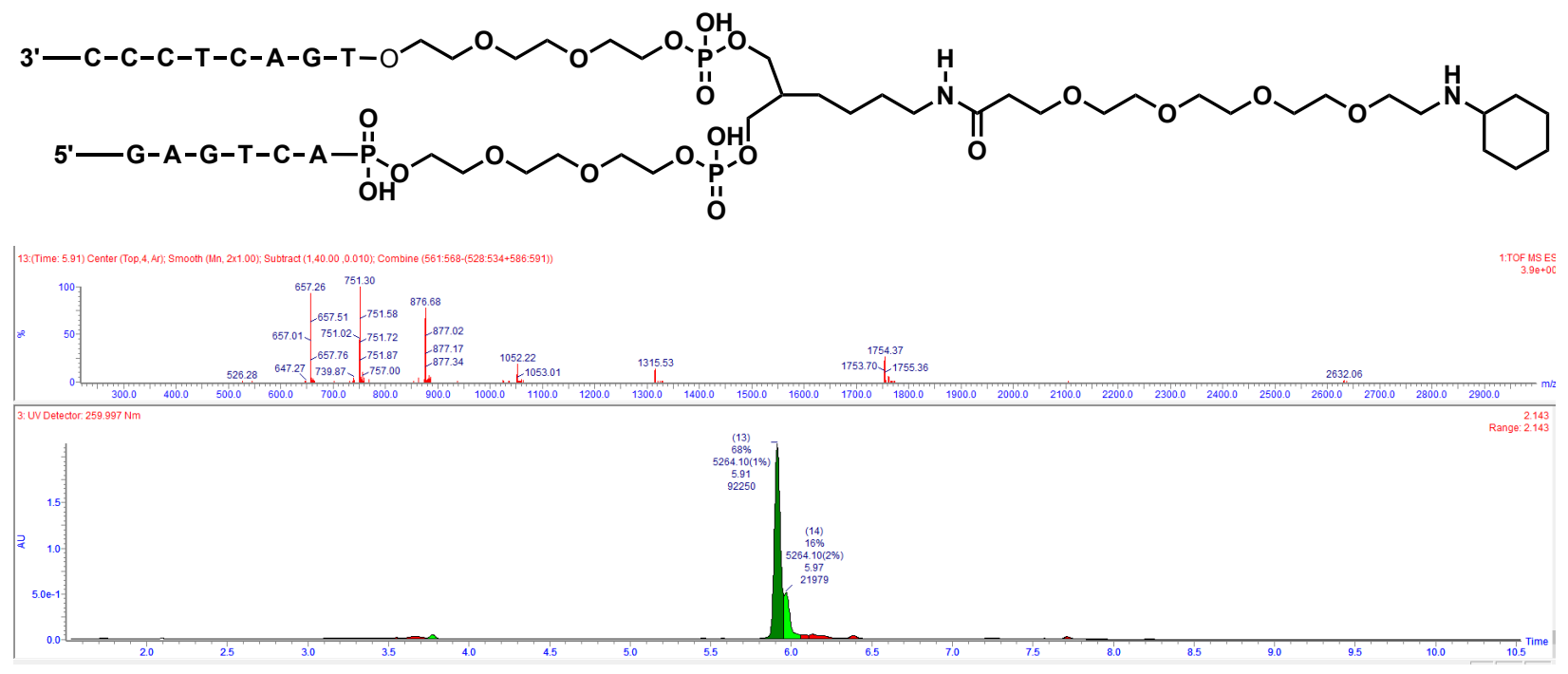


\section{LC Trace and Mass of 77 in tripliacate}

Following General Procedure 7 with 66

Yields: 74, 83, 70\%

Average Yield: $75.66 \%$

SDs: 6.6

Exact masses: 5266.08

Triply charged masses [M-3]/3, caluculated: 1754.36; observed 1754.35, 1754.35, 1754.35
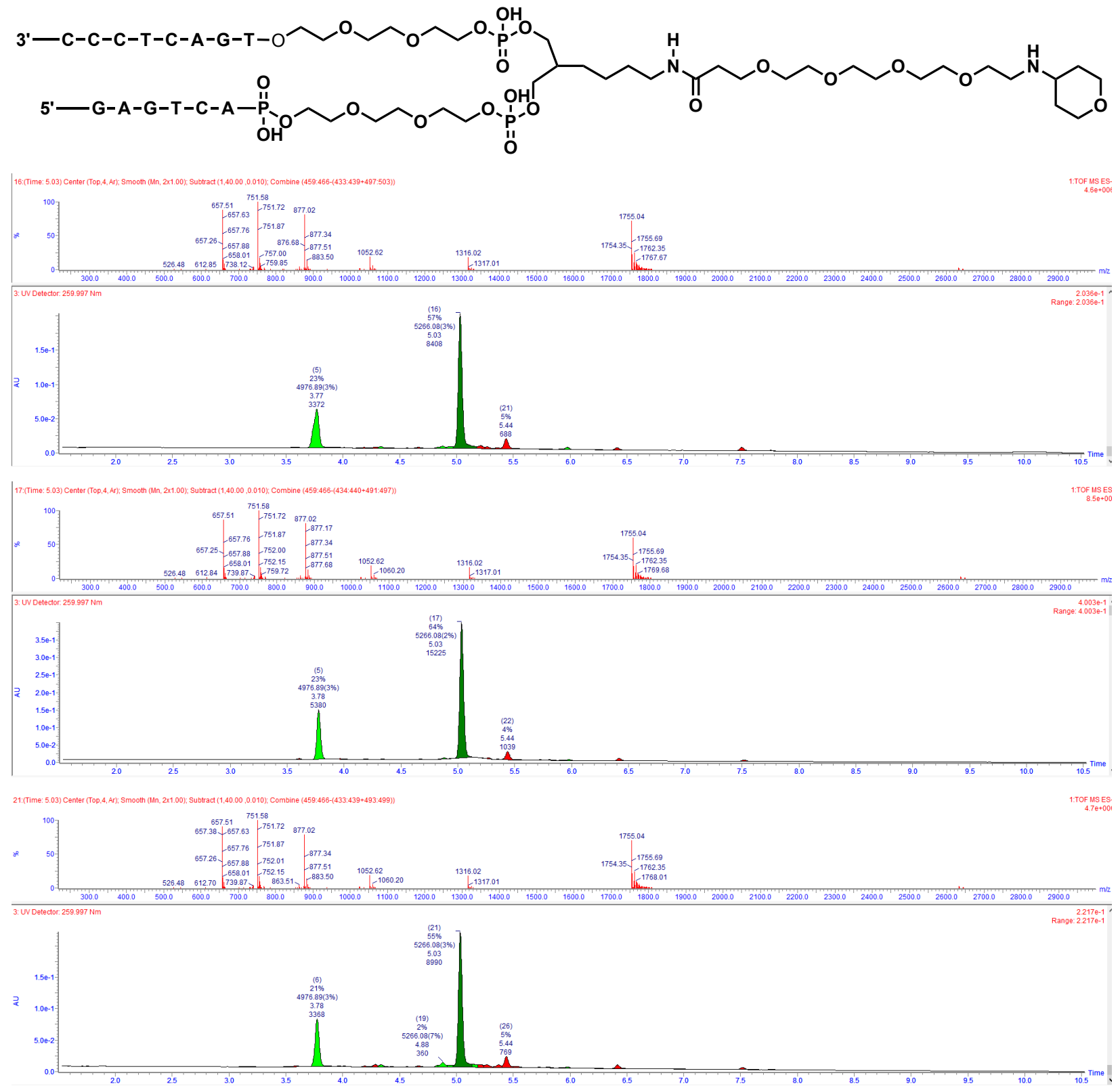


\section{LC Trace and Mass of 77 (Aqueous Reaction)}

Following General Procedure 7 with 66

Yields: $66 \%$

Exact masses: 5266.08

Triply charged masses [M-3]/3, caluculated: 1754.36; observed 1754.35
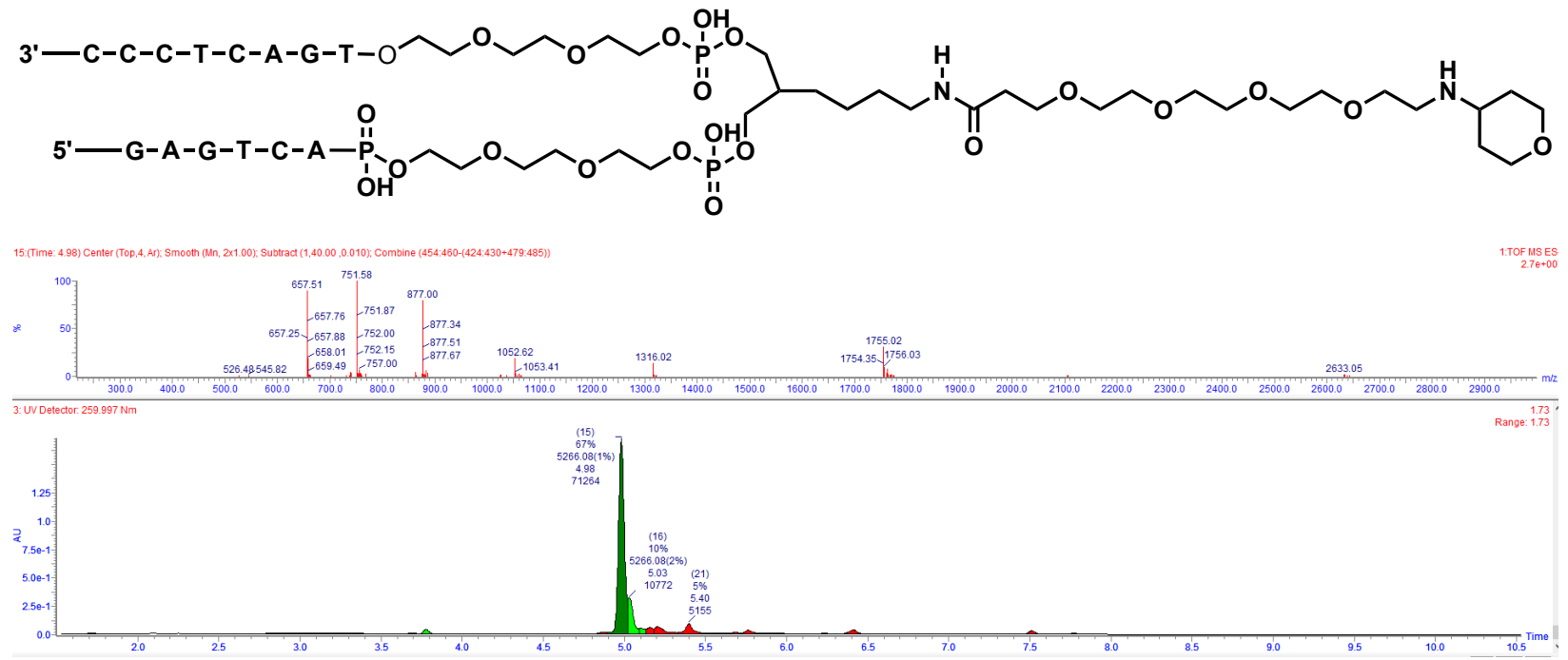


\section{LC Trace and Mass of 78 in tripliacate}

Following General Procedure 7 with 66

Yields: 25, 35, 41\%

Average Yield: 35\%

SDs: 8.5

Exact masses: 5336.12

Triply charged masses [M-3]/3, caluculated: 1777.7; observed 1777.7, 1777.7, 1777.7
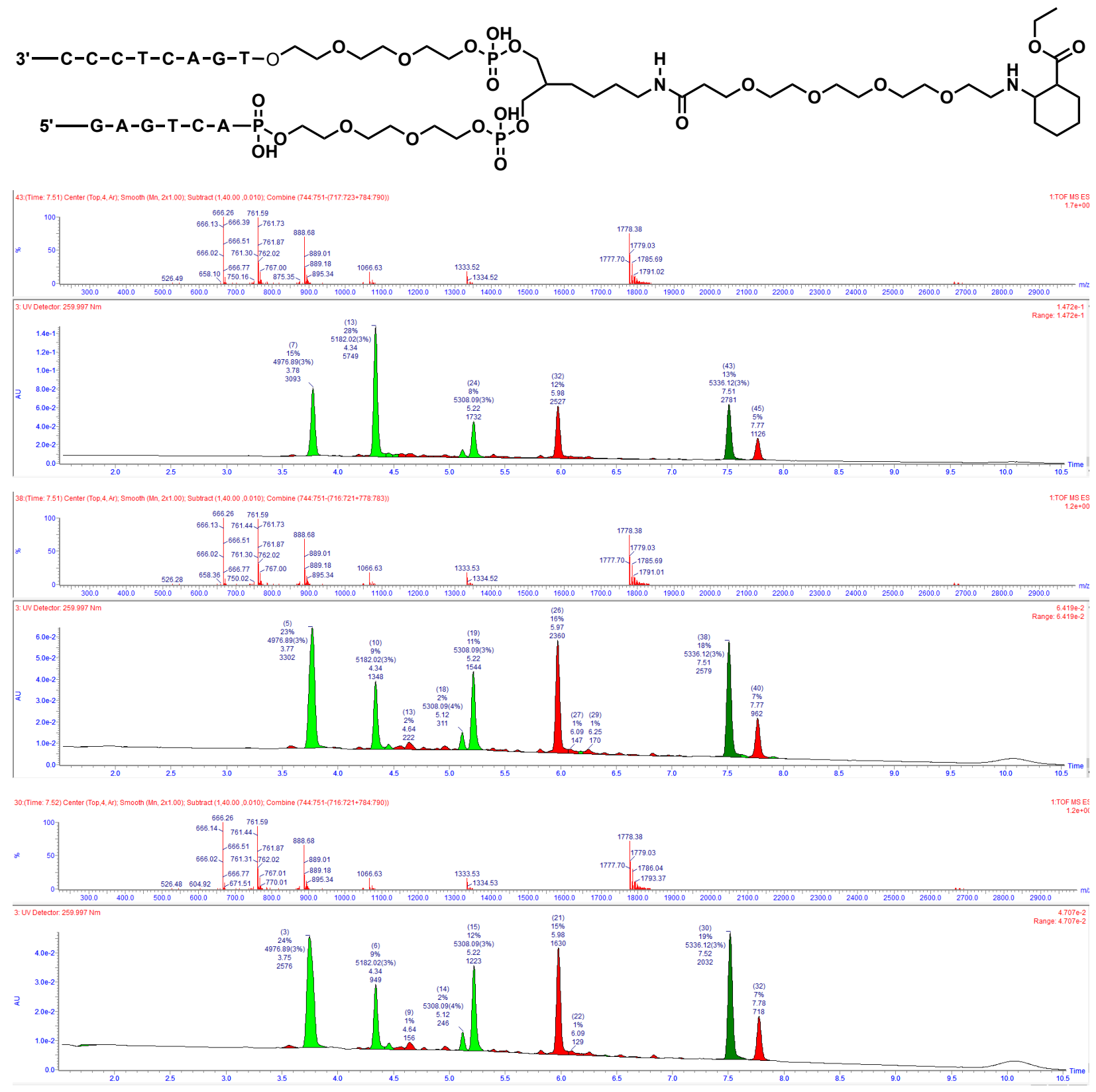


\section{LC Trace and Mass of 78 (Aqueous Reaction)}

Following General Procedure 7 with 66

Yields: $41 \%$

Exact masses: 5336.12

Triply charged masses [M-3]/3, caluculated: 1754.36 ; observed 1777.7
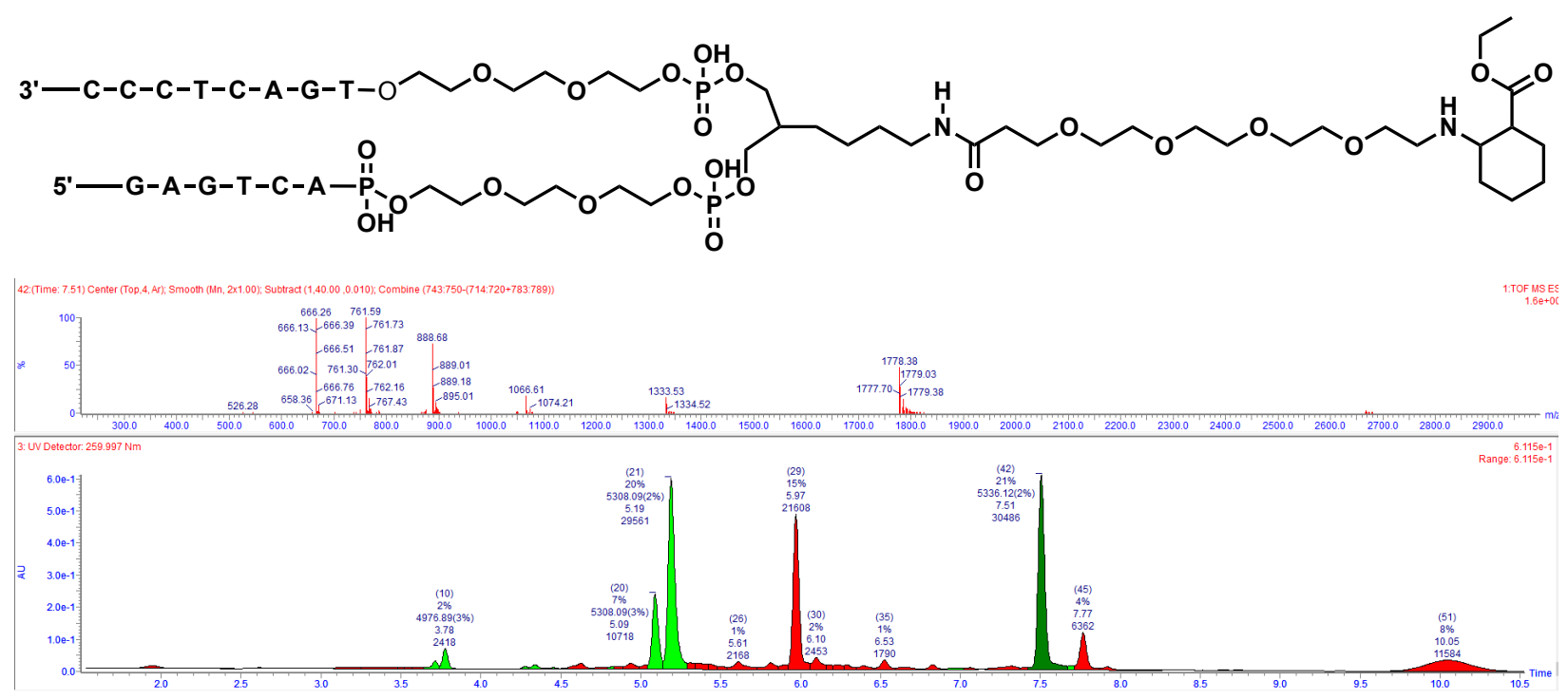


\section{LC Trace and Mass of 79 in tripliacate}

Following General Procedure 7 with 66

Yields: 78, 84, 68\%

Average Yield: $76.66 \%$

SDs: 8.0

Exact masses: 5278.12

Triply charged masses [M-3]/3, caluculated: 1758.37; observed 1758.37, 1758.37, 1758.37
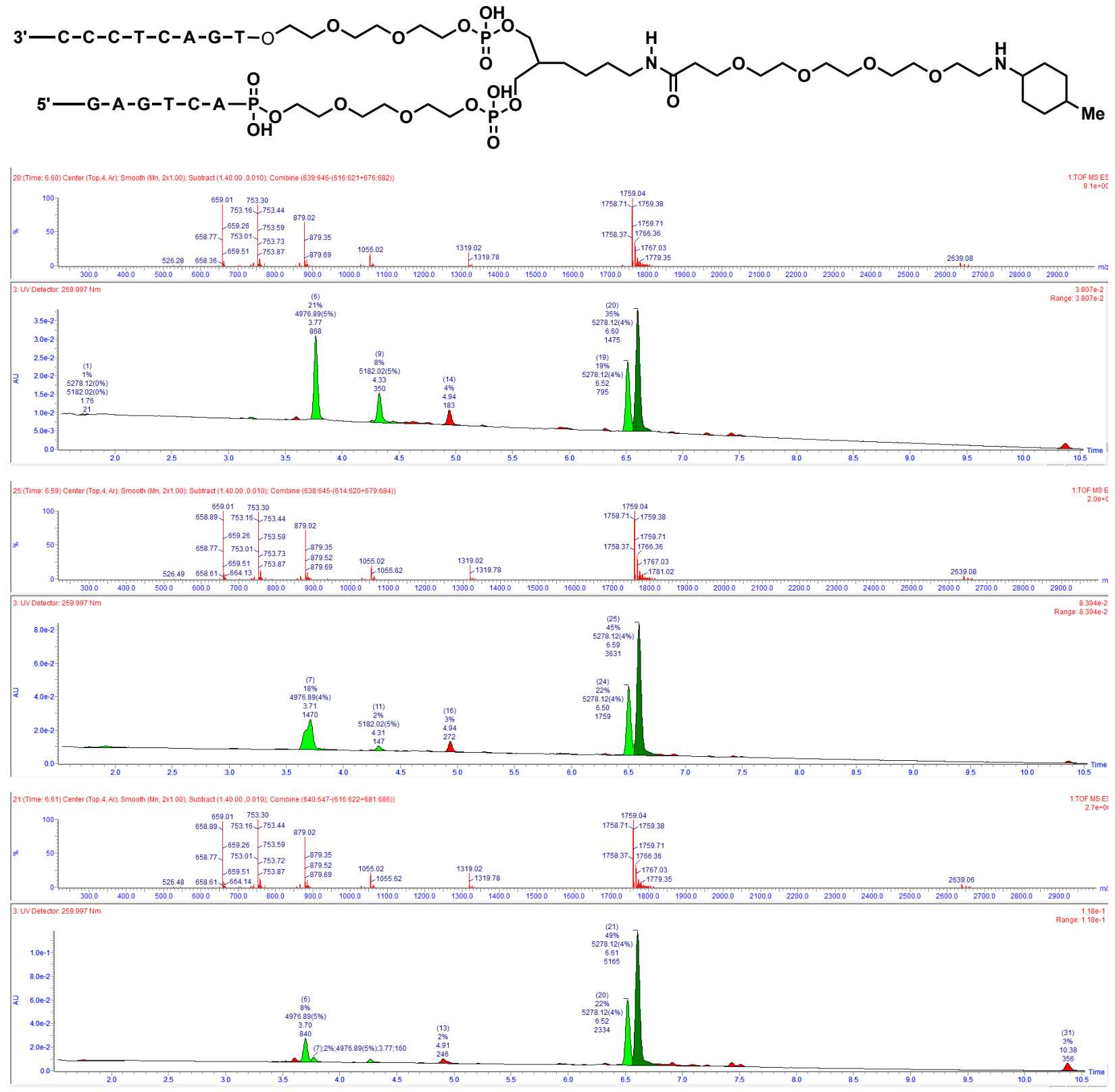


\section{LC Trace and Mass of 79 (Aqueous Reaction)}

Following General Procedure 7 with 66

Yields: $83 \%$

Exact masses: 5278.12

Triply charged masses [M-3]/3, caluculated: 1758.37; observed 1758.37,
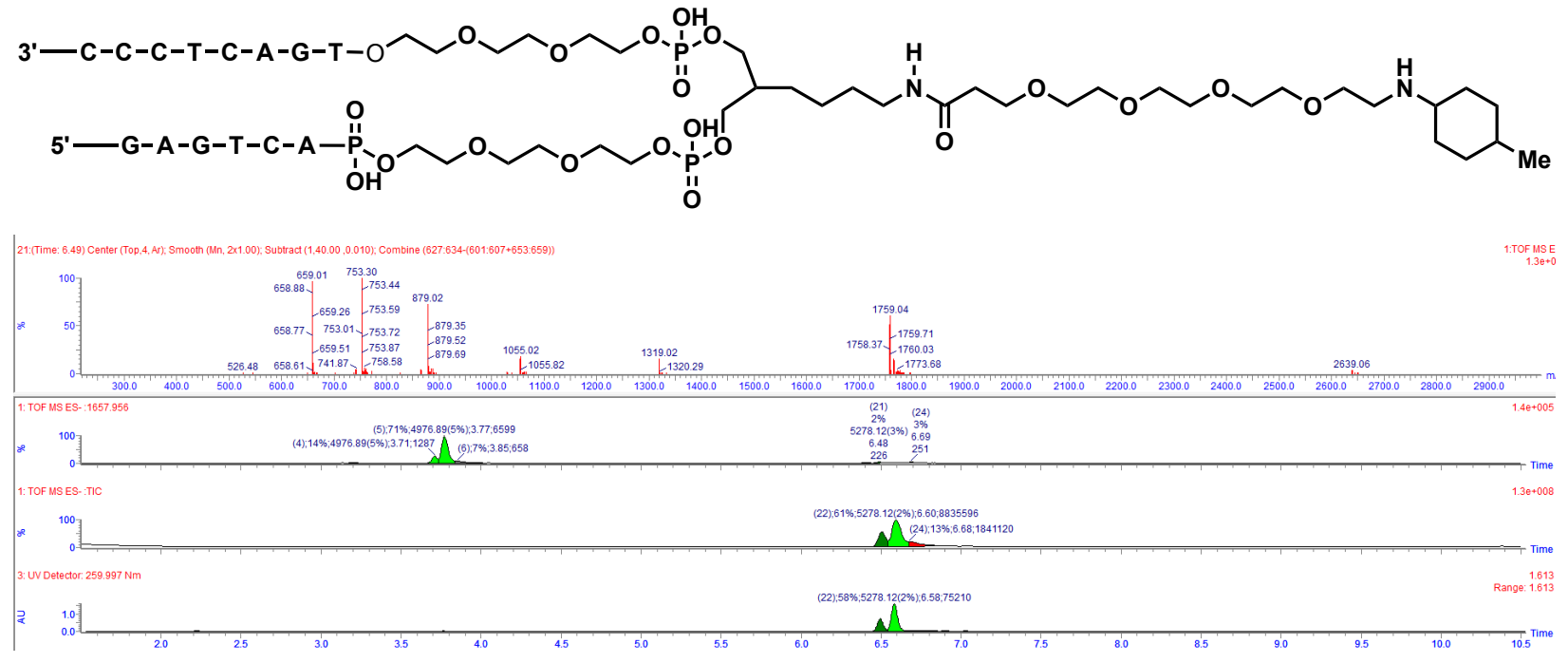


\section{LC Trace and Mass of 80 in tripliacate}

Following General Procedure 7 with 66

Yields: 62, 55, 61\%

Average Yield: 59.33\%

SDs: 3.8

Exact masses: 5320.17

Triply charged masses [M-3]/3, caluculated: 1772.39; observed $1772.39,1772.39$, 1772.39
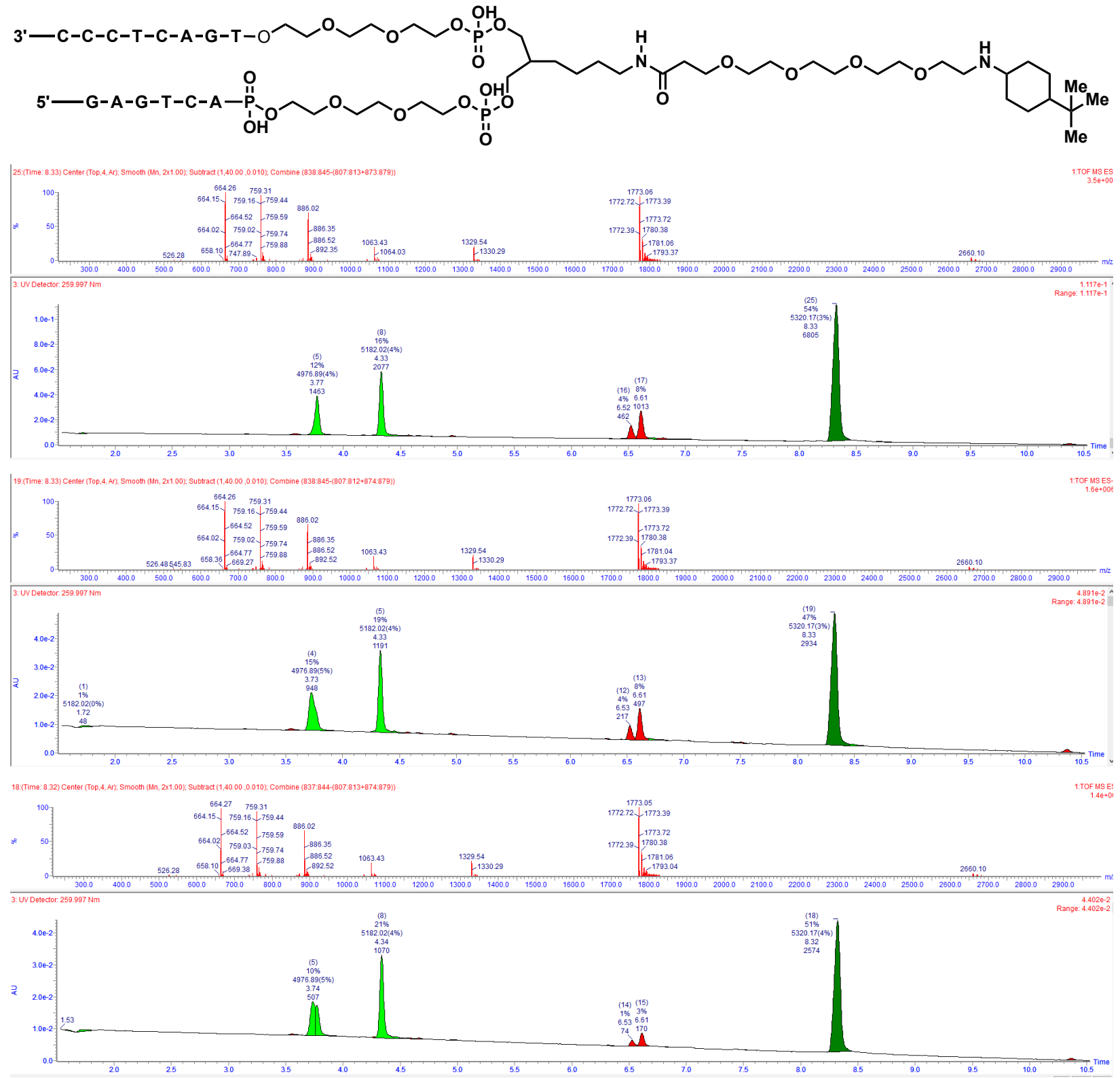


\section{LC Trace and Mass of 80 (Aqueous Reaction)}

Following General Procedure 7 with 66

Yields: $85 \%$

Exact masses: 5320.17

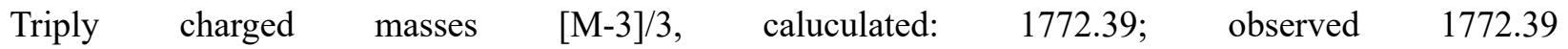
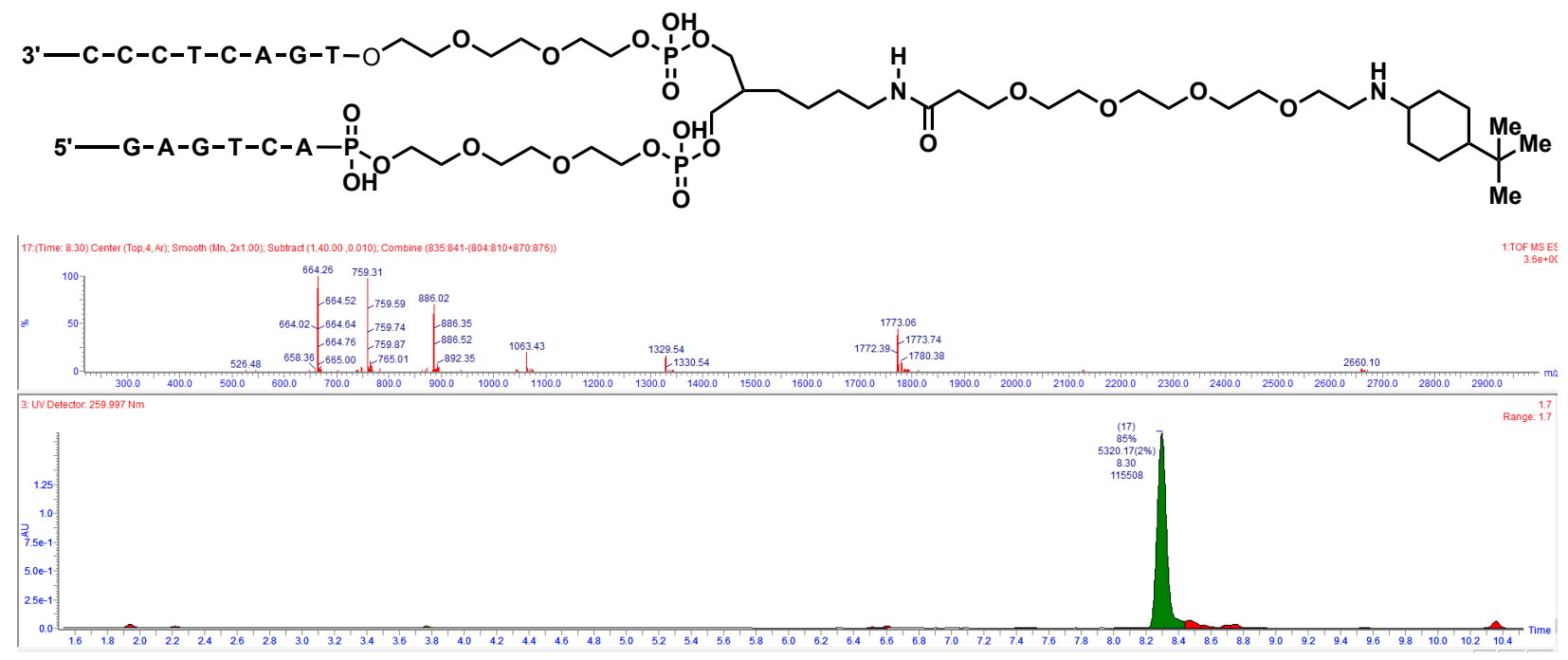


\section{LC Trace and Mass of 81 in tripliacate}

Following General Procedure 7 with 66

Yields: $83,75,80 \%$

Average Yield: $79.33 \%$

SDs: 4.0

Exact masses: 5365.15

Triply charged masses [M-3]/3, caluculated: 1787.38; observed 1787.37, 1787.37, 1787.37
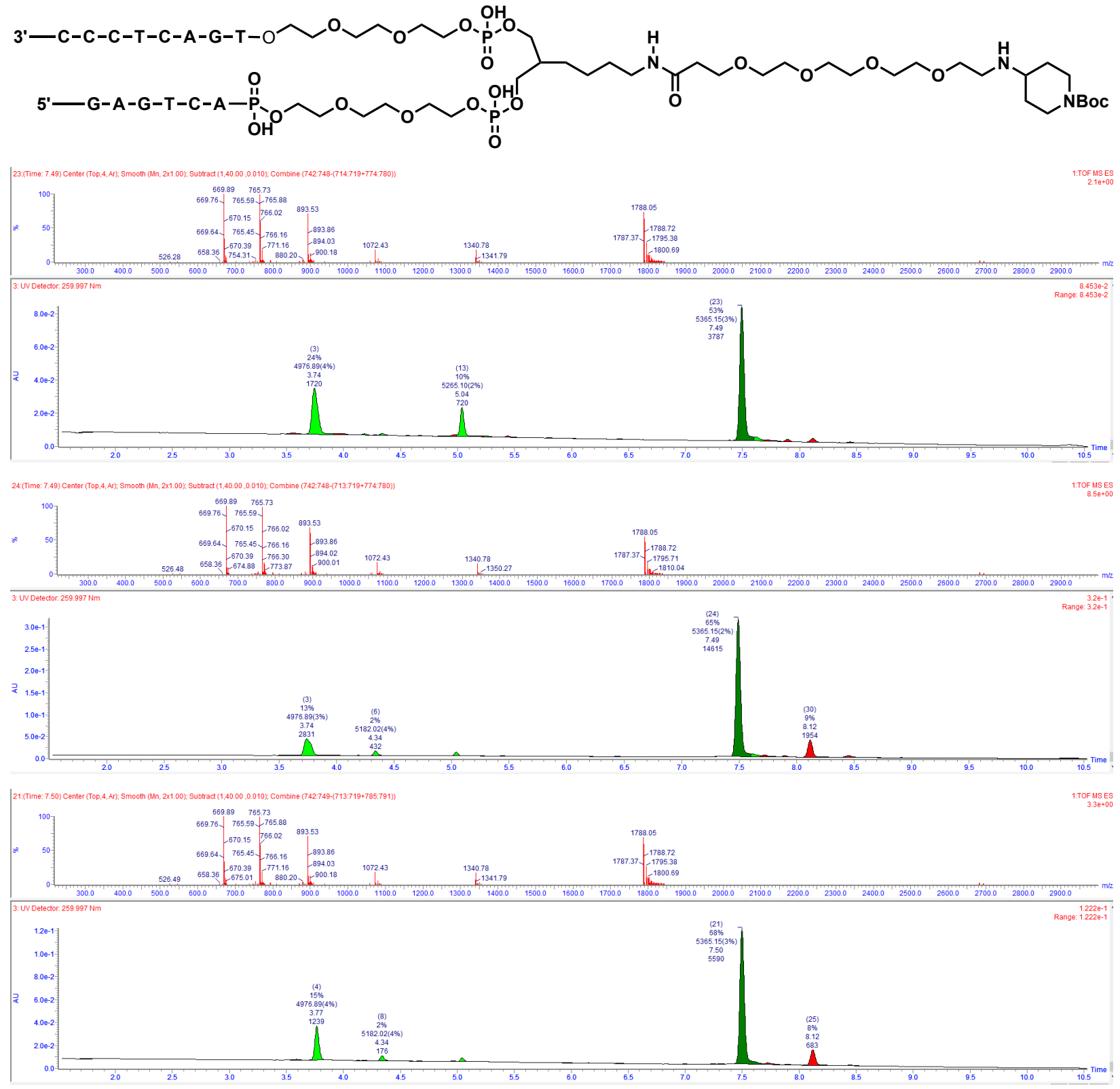


\section{LC Trace and Mass of 81 (Aqueous Reaction)}

Following General Procedure 7 with 66

Yields: $86 \%$

Exact masses: 5365.15

Triply charged masses [M-3]/3, caluculated: 1787.38; observed 1787.37
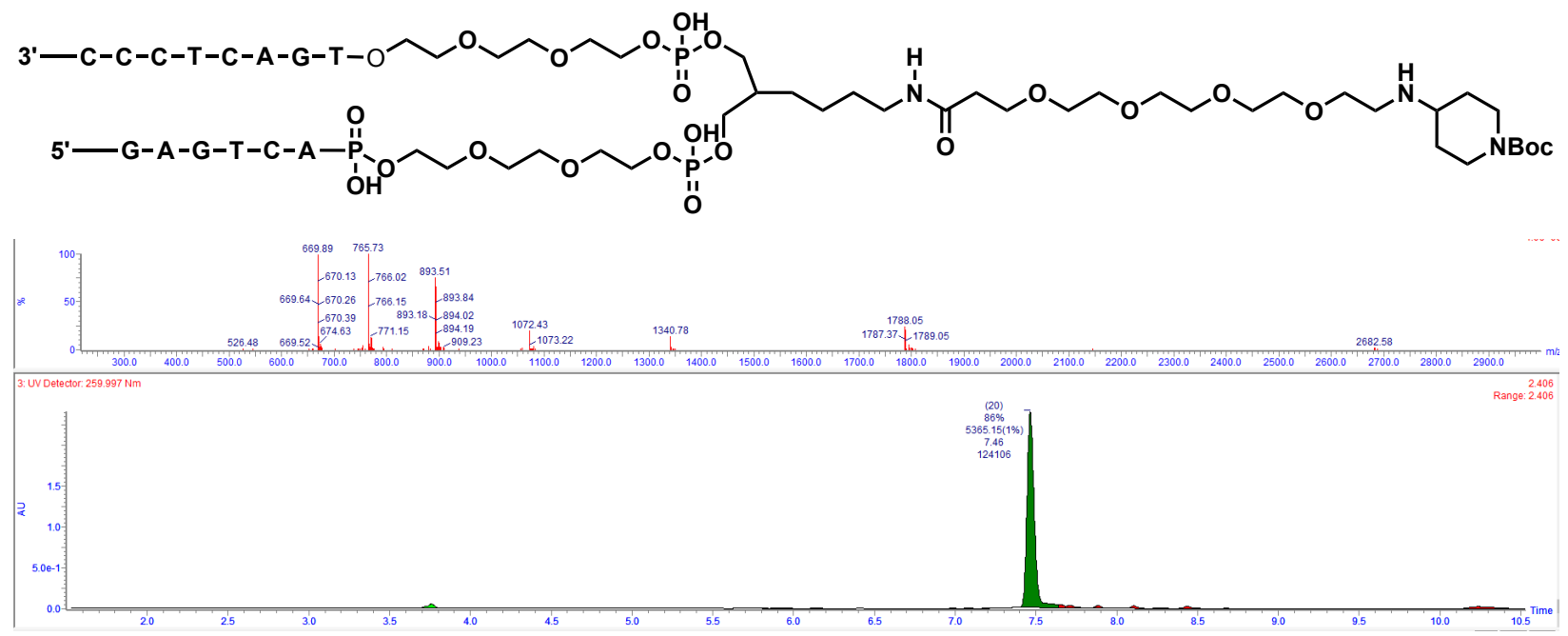


\section{LC Trace and Mass of 82 in tripliacate}

Following General Procedure 7 with 66

Yields: 23, 27, 31\%

Average Yield: 27\%

SDs: 4.0

Exact masses: 5334.06

Triply charged masses [M-3]/3, caluculated: 1777.02; observed 1777.01, 1777.01, 1777.01
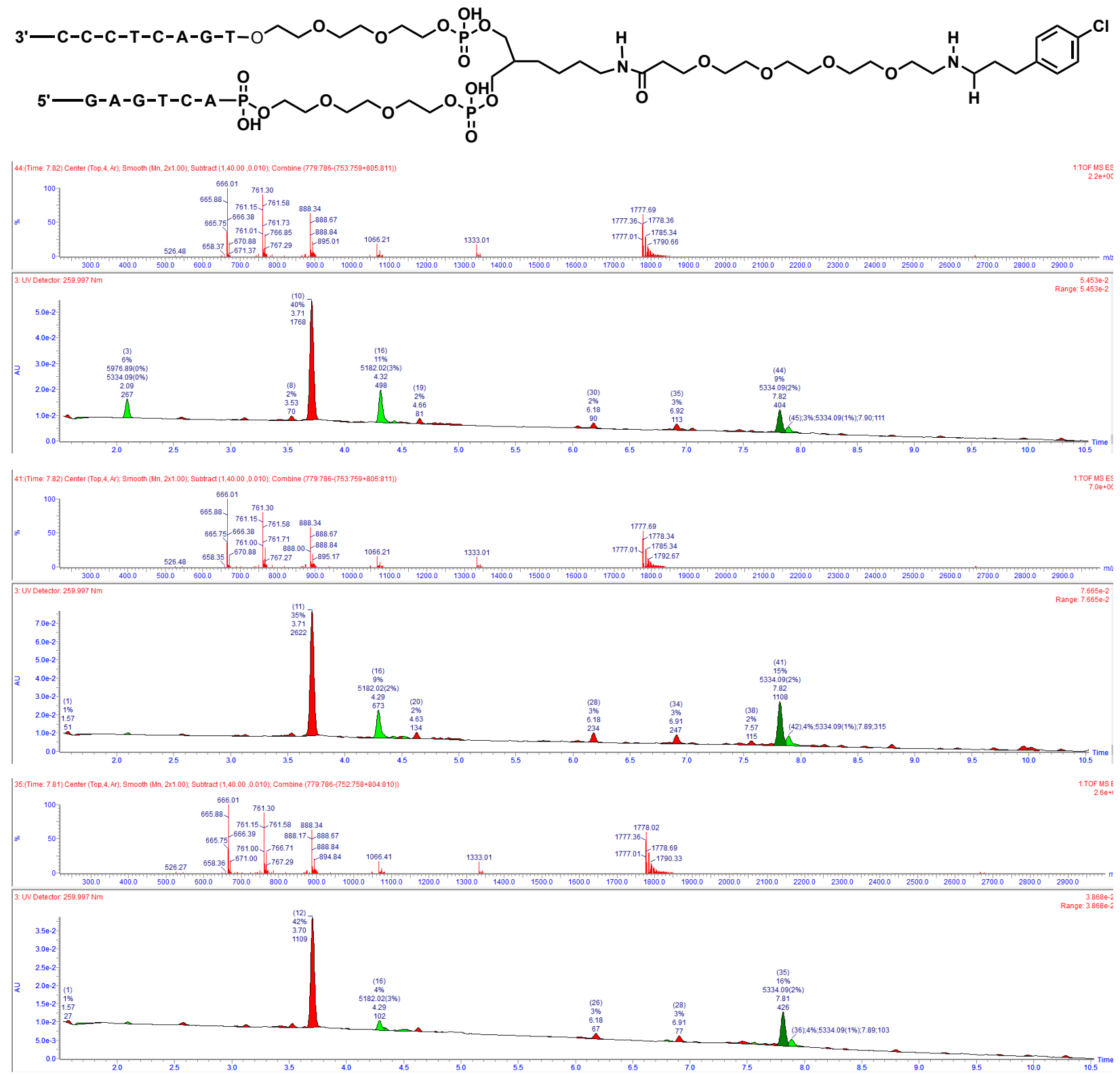


\section{LC Trace and Mass of 82 (Aqueous Reaction)}

Following General Procedure 7 with 66

Yields: $11 \%$

Exact masses: 5334.06

Triply charged masses $[\mathrm{M}-3] / 3, \quad$ caluculated: $1777.01, \quad$ Obs. 1777.01
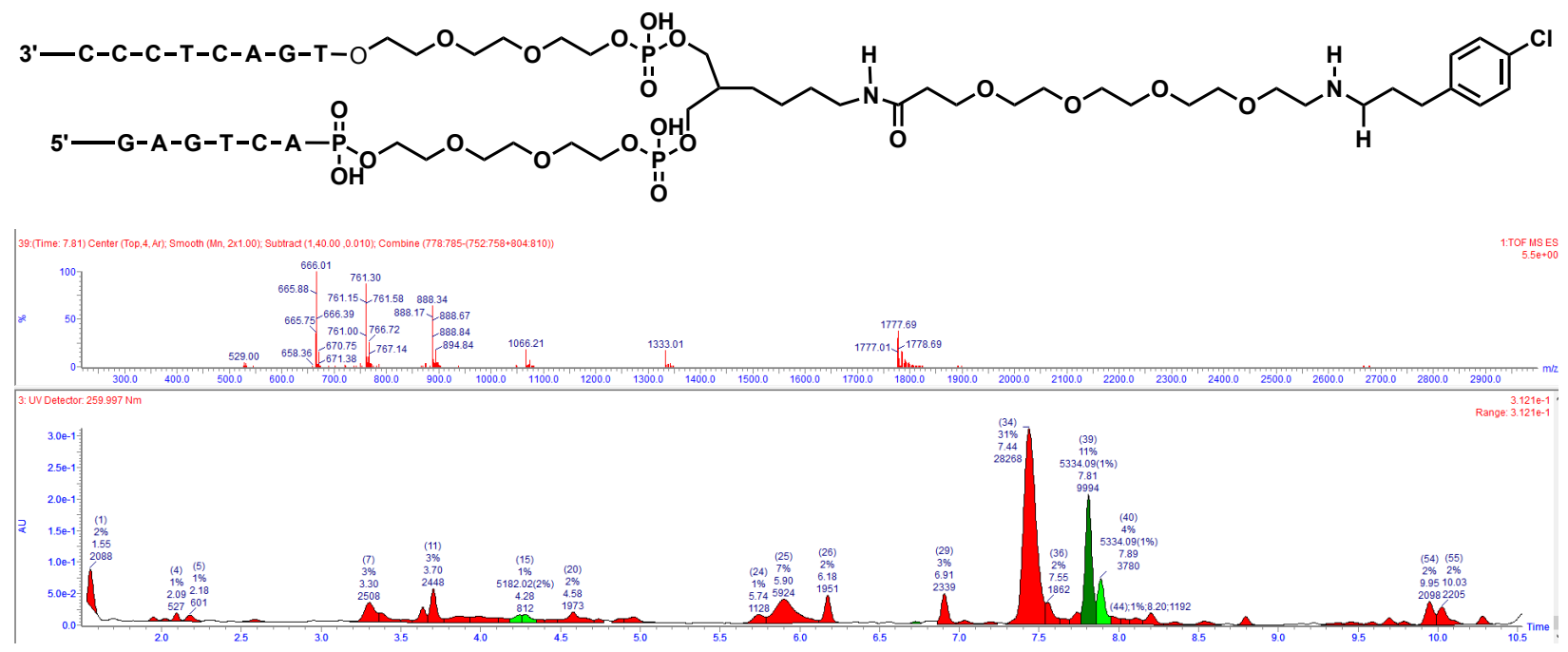


\section{LC Trace and Mass of 83 (Aqueous Reaction)}

Following General Procedure 7 with 66

Yields: $7 \%$

Exact masses: 5432.92

Triply charged masses [M-3]/3, caluculated: 1809.97; observed 1787.37
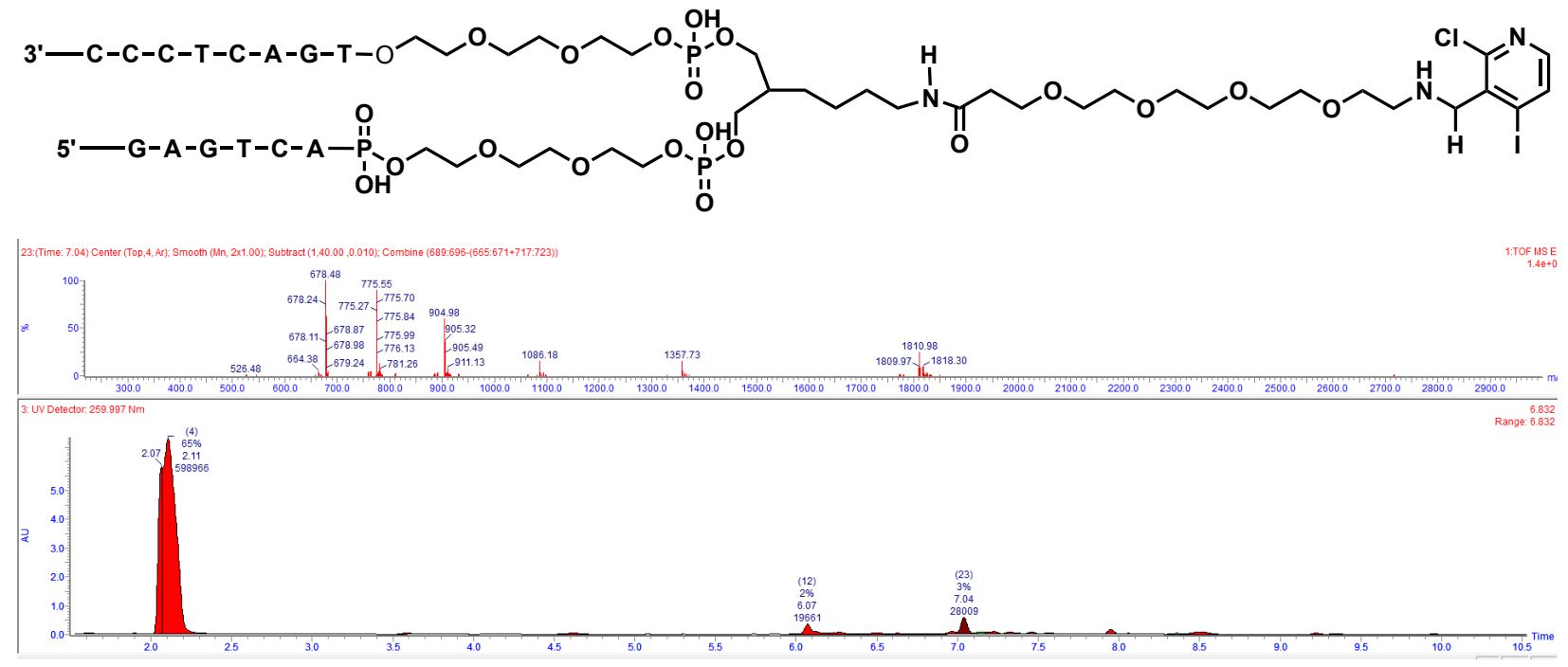


\section{LC Trace and Mass of 83 in tripliacate}

Following General Procedure 7 with 66

Yields: 43, 36, 40\%

Average Yield: $39.66 \%$

SDs: 3.5

Exact masses: 5432.92

Triply charged masses [M-3]/3, caluculated: 1809.97; observed 1809.97 1809.97 1809.97
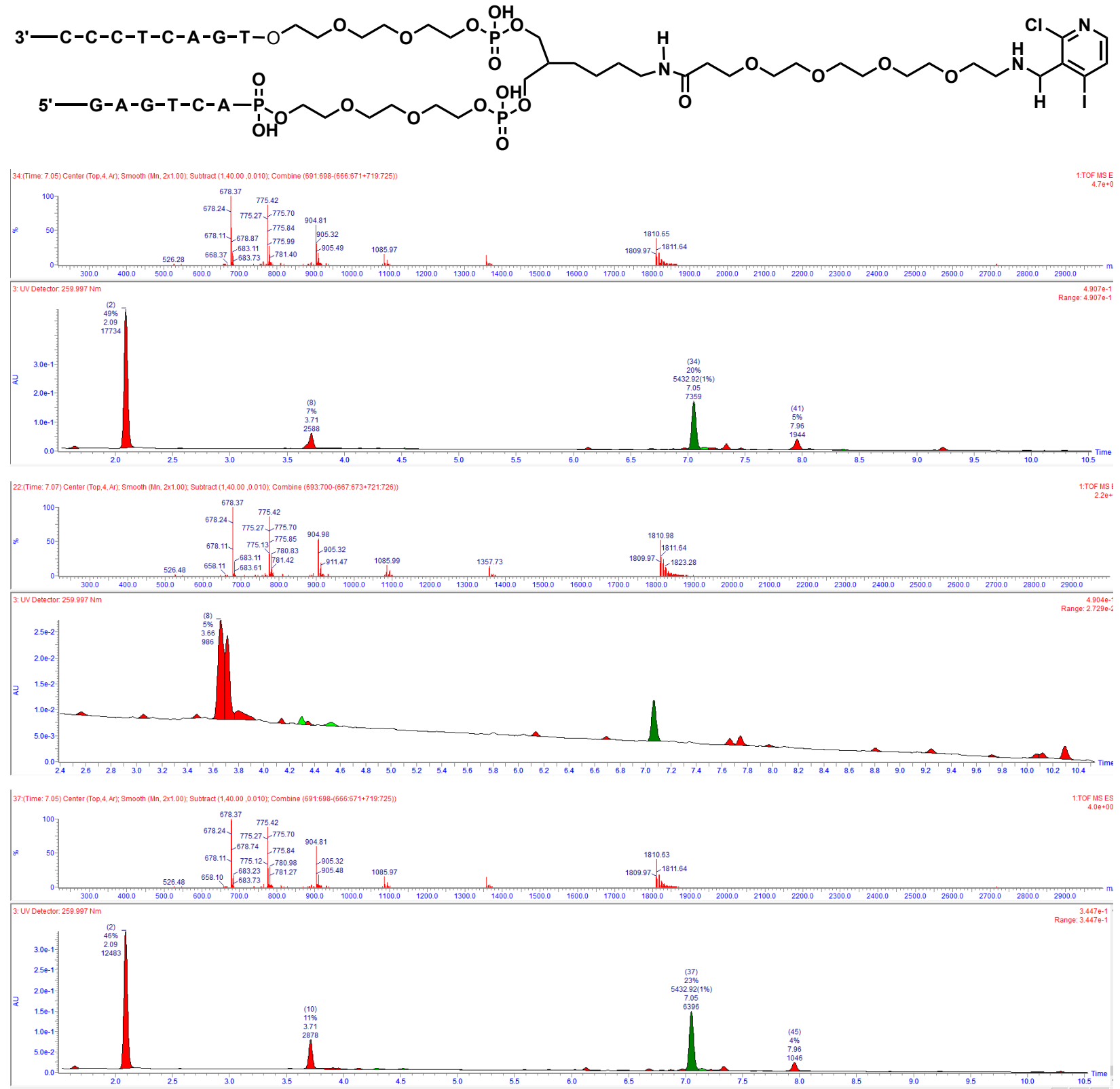


\section{LC Trace and Mass of 83 (Aqueous Reaction)}

Following General Procedure 7 with 66

Yields: $7 \%$

Exact masses: 5432.92

Triply charged masses [M-3]/3, caluculated: 1809.97; observed 1787.37
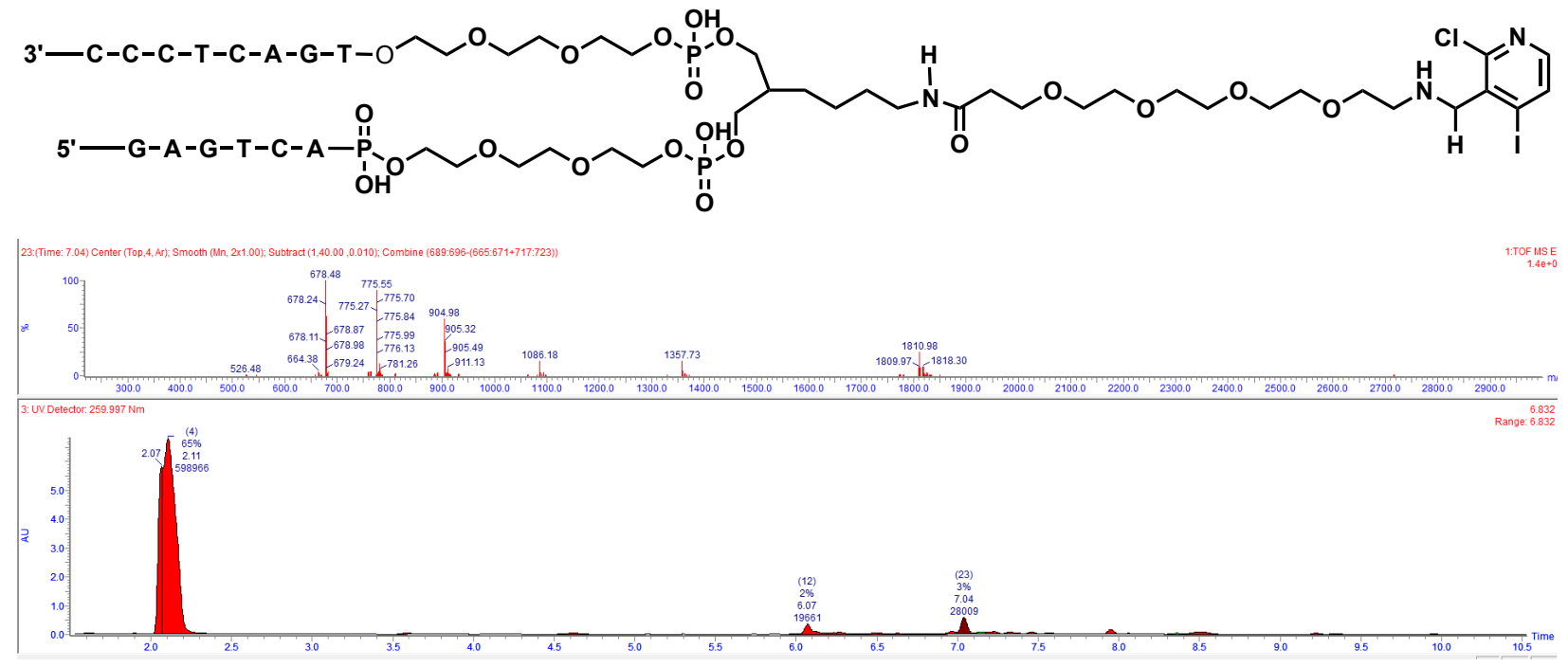


\section{LC Trace and Mass of 84 in tripliacate}

Following General Procedure 7 with 66

Yields: 32, 52, 64\%

Average Yield: $49.33 \%$

SDs: 16.2

Exact masses: 5316.13

Triply charged masses [M-3]/3, caluculated: 1771.04; observed 1771.05, $1771.05,1771.05$
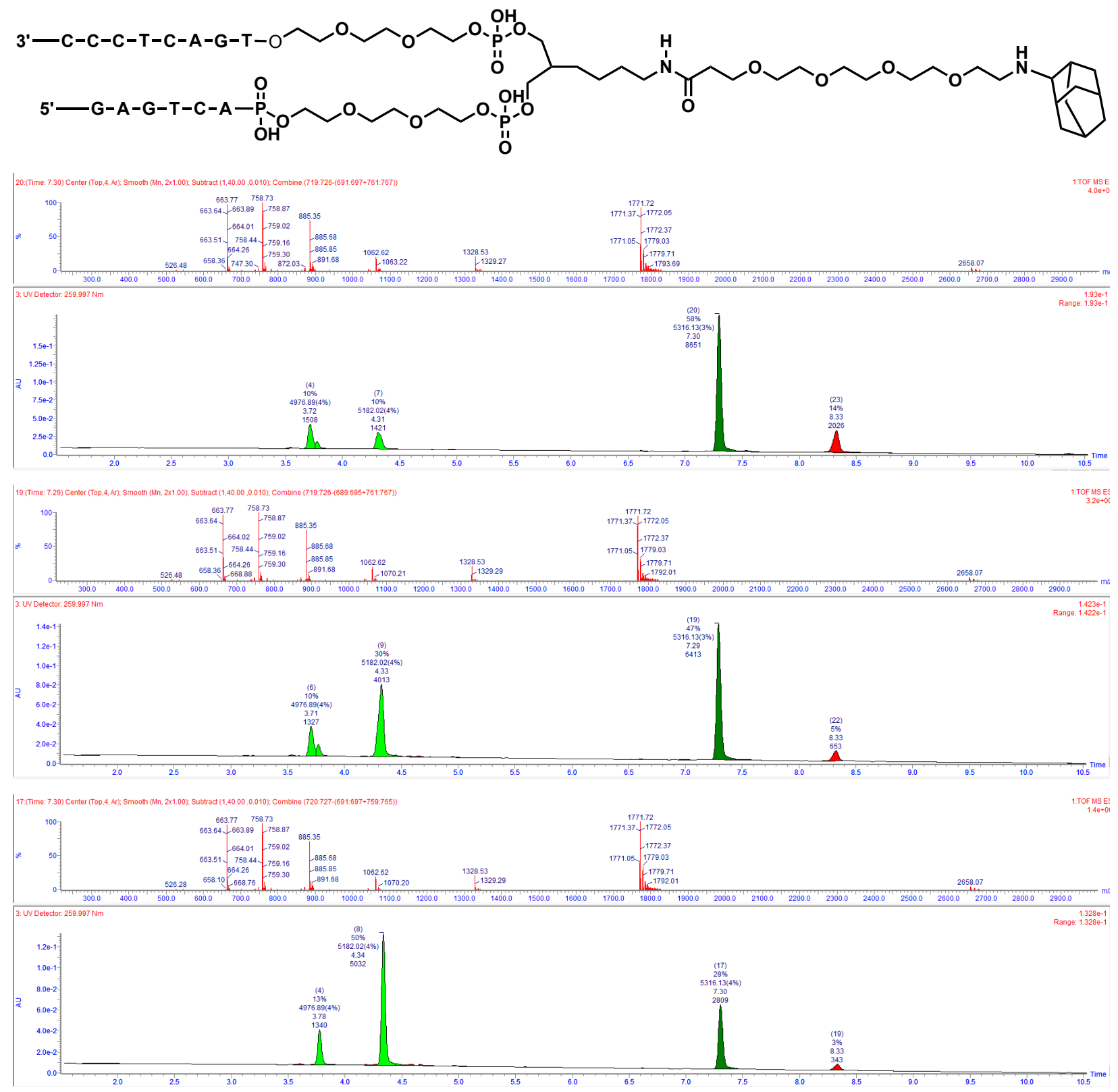


\section{LC Trace and Mass of 84 (Aqueous Reaction)}

Following General Procedure 7 with 66

Yields: $82 \%$

Exact masses: 5316.13

Triply charged masses [M-3]/3, caluculated: 1771.04; observed 1771.05
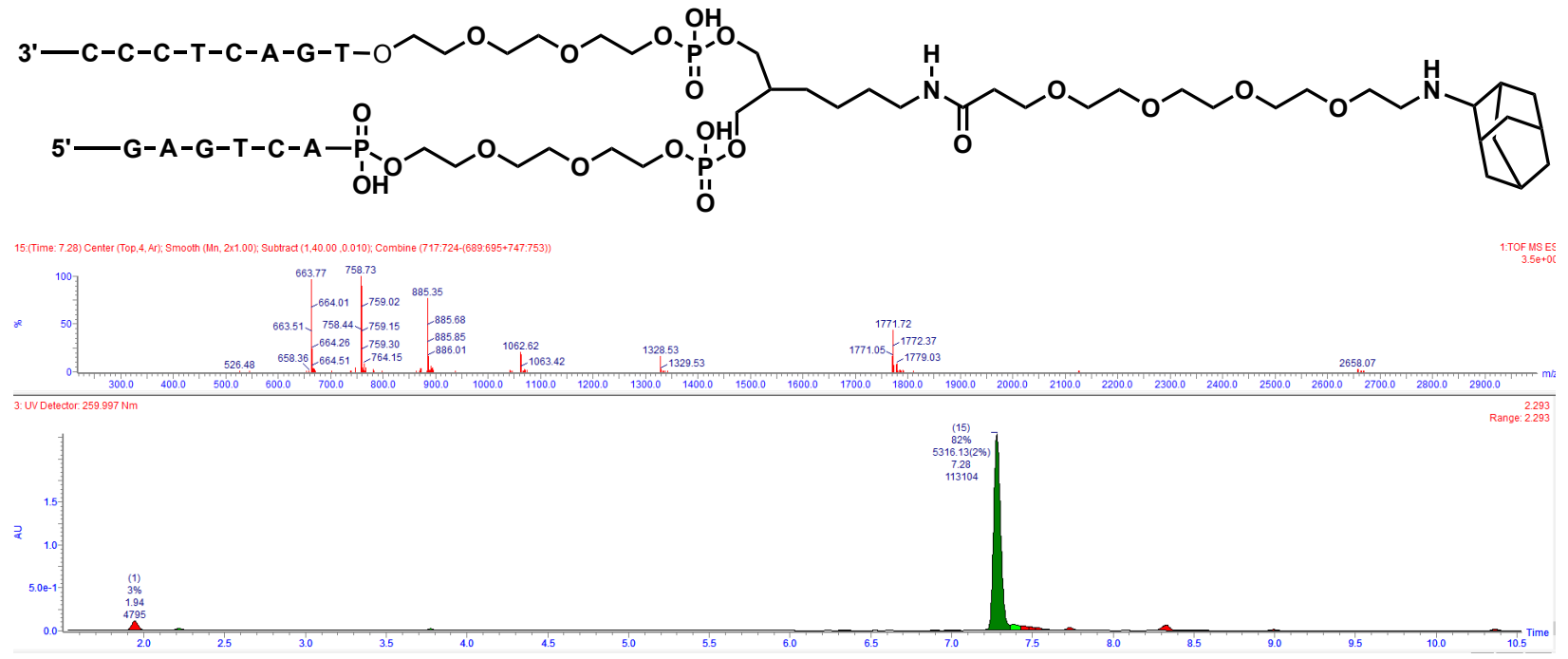


\section{LC Trace and Mass of 85 in tripliacate}

Following General Procedure 7 with 66

Yields: 43, 60, 64\%

Average Yield: 55.66\%

SDs: 11.2

Exact masses: 5472.02

Triply charged masses [M-3]/3, caluculated: 1823.00; observed 1823.001823 .001823 .00
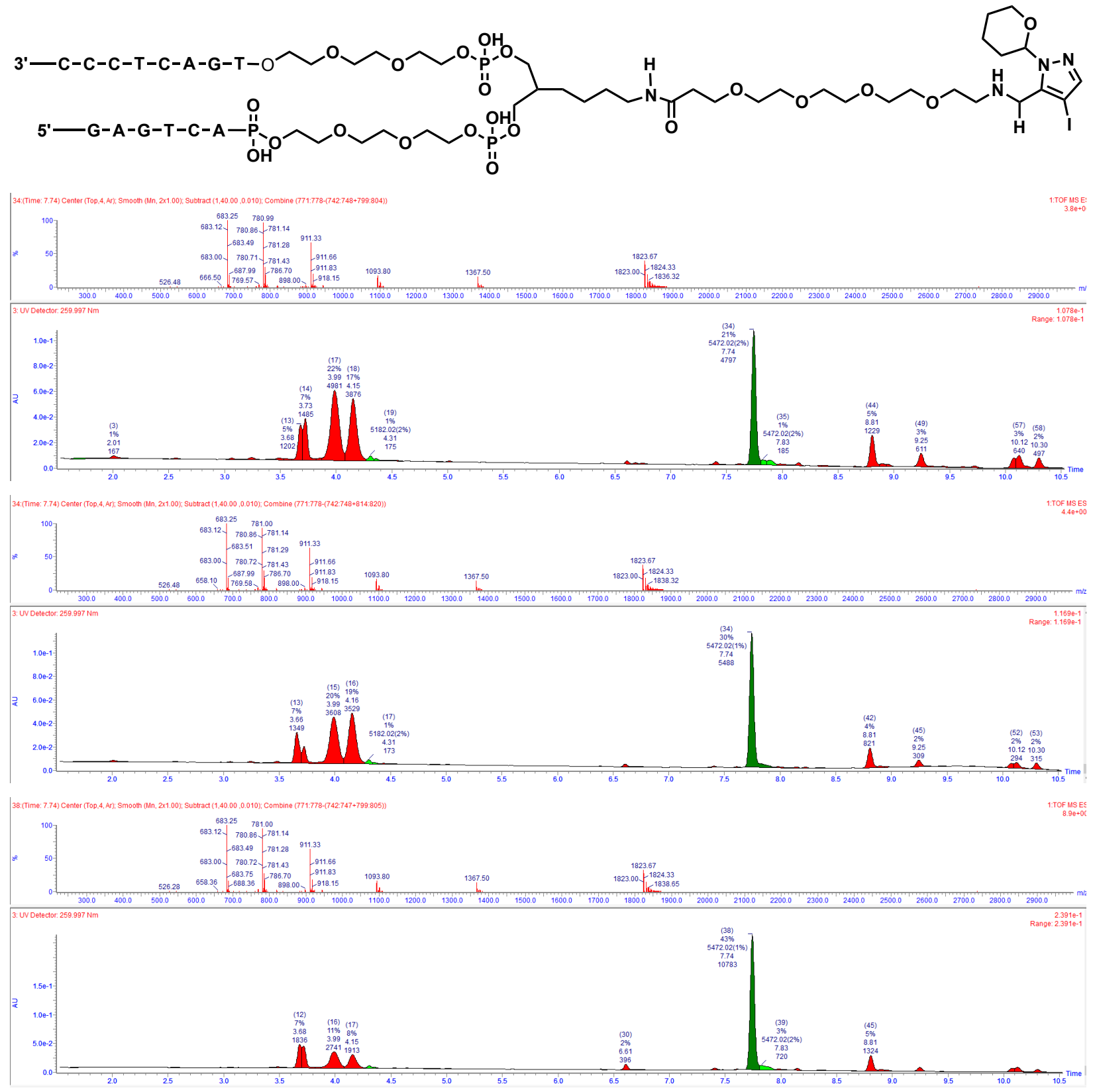


\section{LC Trace and Mass of 85 (Aqueous Reaction)}

Following General Procedure 7 with 66

Yields: $39 \%$

Exact masses: 5472.02

Triply charged masses [M-3]/3, caluculated: 1823.00; observed 1823.00
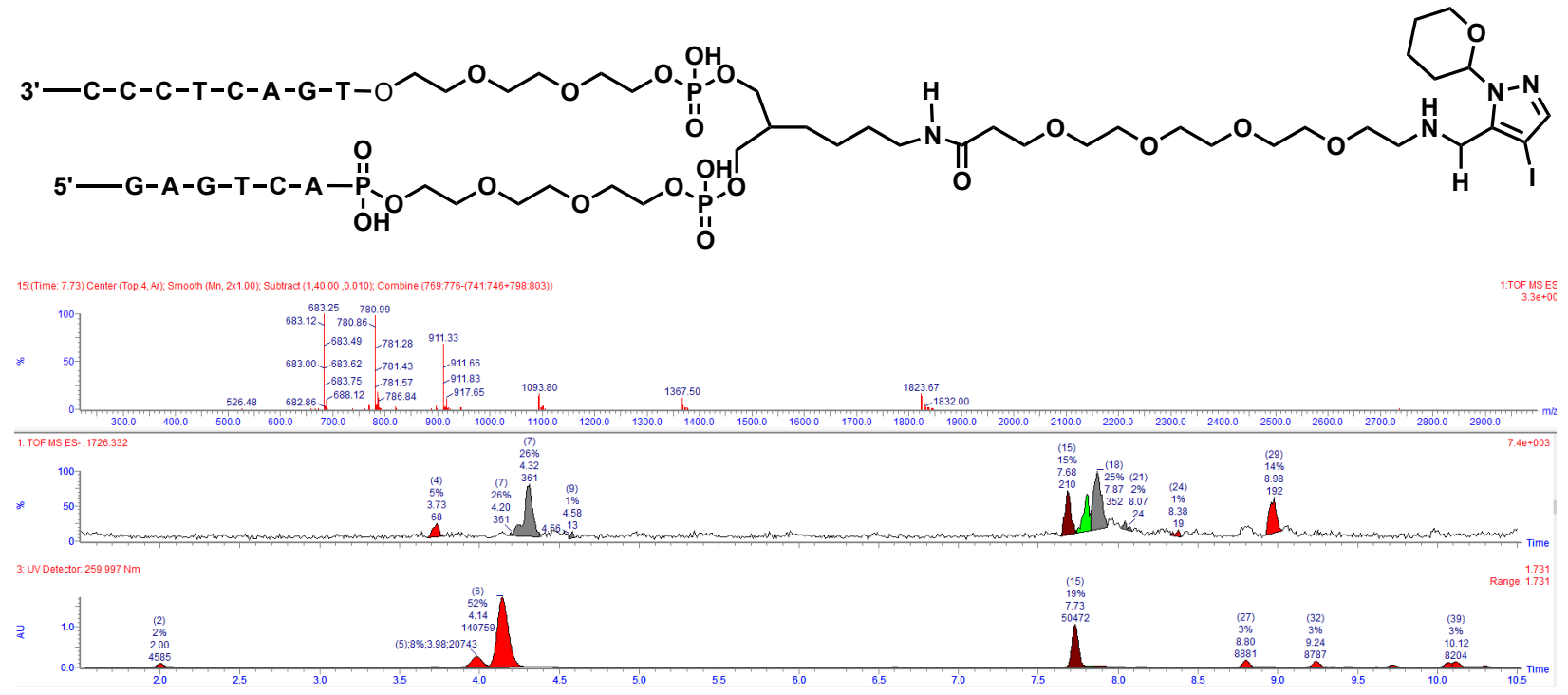


\section{LC Trace and Mass of 86 in tripliacate}

Following General Procedure 7 with 66

Yields: 15, 24, 29\%

Average Yield: $22.66 \%$

SDs: 7.1

Exact masses: 5323.08

Triply charged masses [M-3]/3, caluculated: 1773.36; observed 1773.36, 1773.36, 1773.36
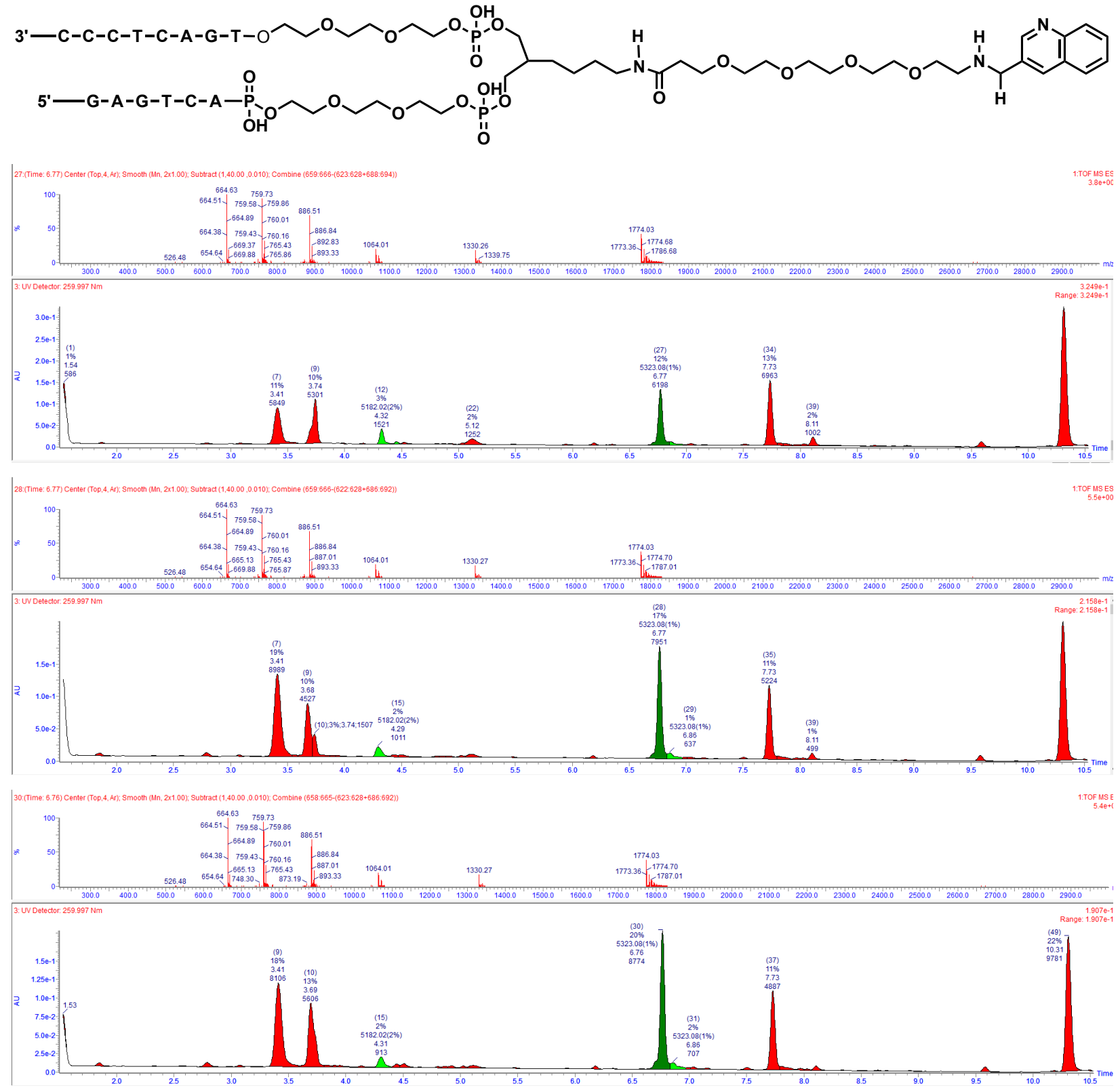


\section{LC Trace and Mass of 86 (Aqueous Reaction)}

Following General Procedure 7 with 66

Yields: $10 \%$

Exact masses: 5323.08

Triply charged masses [M-3]/3, caluculated: 1773.36; observed 1773.36
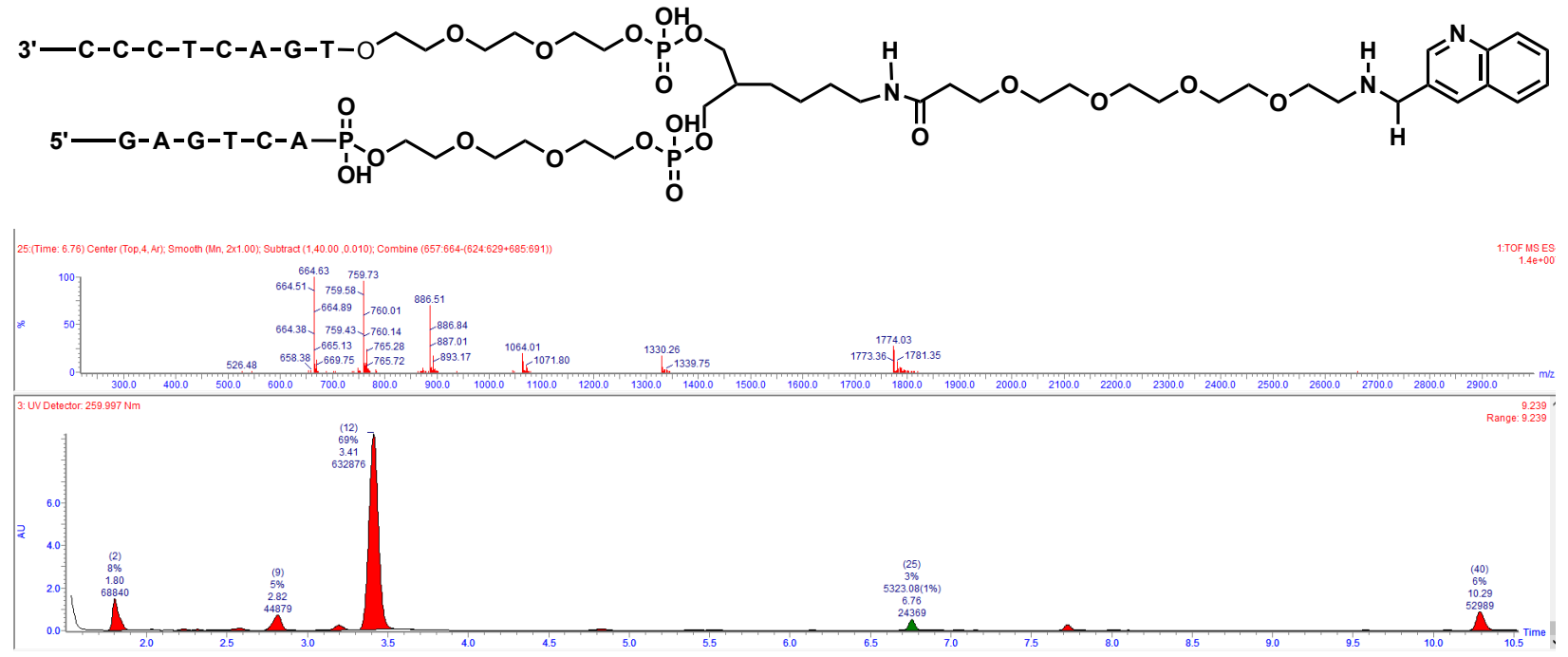


\section{LC Trace and Mass of 87 in tripliacate}

Following General Procedure 7 with 66

Yields: 37, 49, 55\%

Average Yield: $47 \%$

SDs: 9.2

Exact masses: 5413.19

Triply charged masses [M-3]/3, caluculated: 1803.4; observed 1803.4, 1803.4, 1803.4
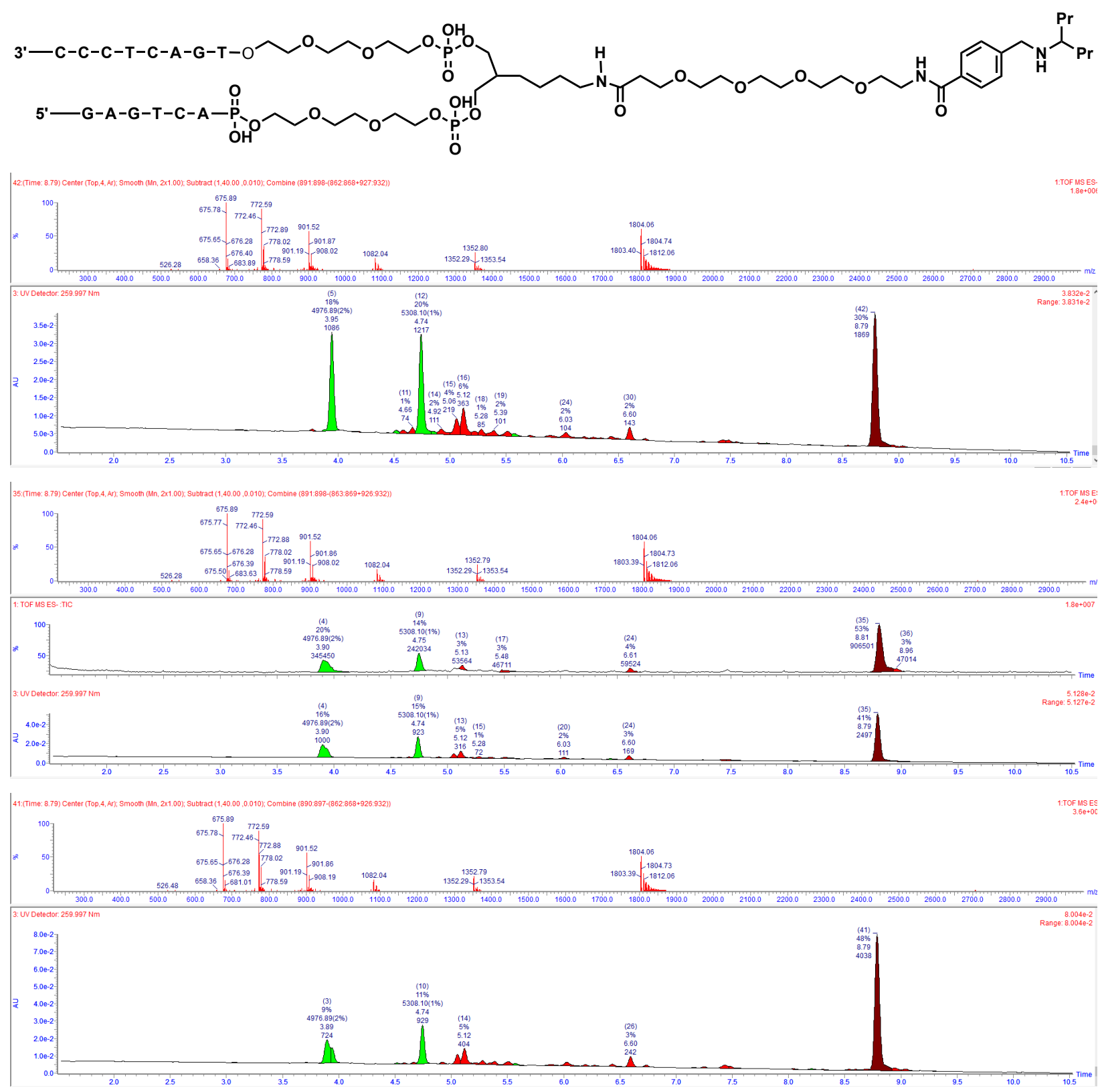


\section{LC Trace and Mass of 87 (Aqueous Reaction)}

Following General Procedure 7 with SI-11

Yields: $51 \%$

Exact masses: 5413.19

Triply charged masses [M-3]/3, caluculated: 1803.4; observed 1803.4
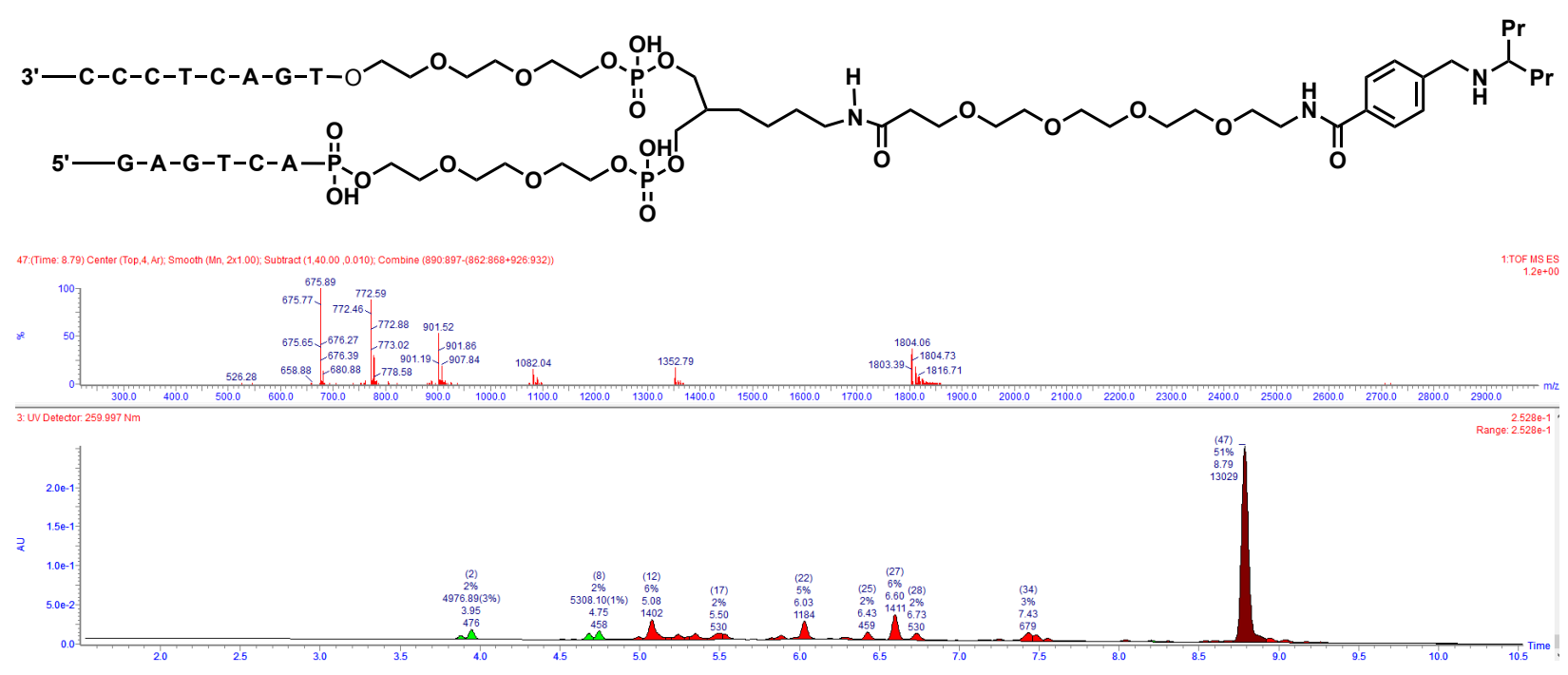


\section{LC Trace and Mass of 88 in tripliacate}

Following General Procedure 7 with 66

Yields: 40, 59, 55\%

Average Yield: $51.33 \%$

SDs: 10

Exact masses: 5363.17

Triply charged masses [M-3]/3, caluculated: 1786.7;; observed 1786.7, 1786.7, 1786.7
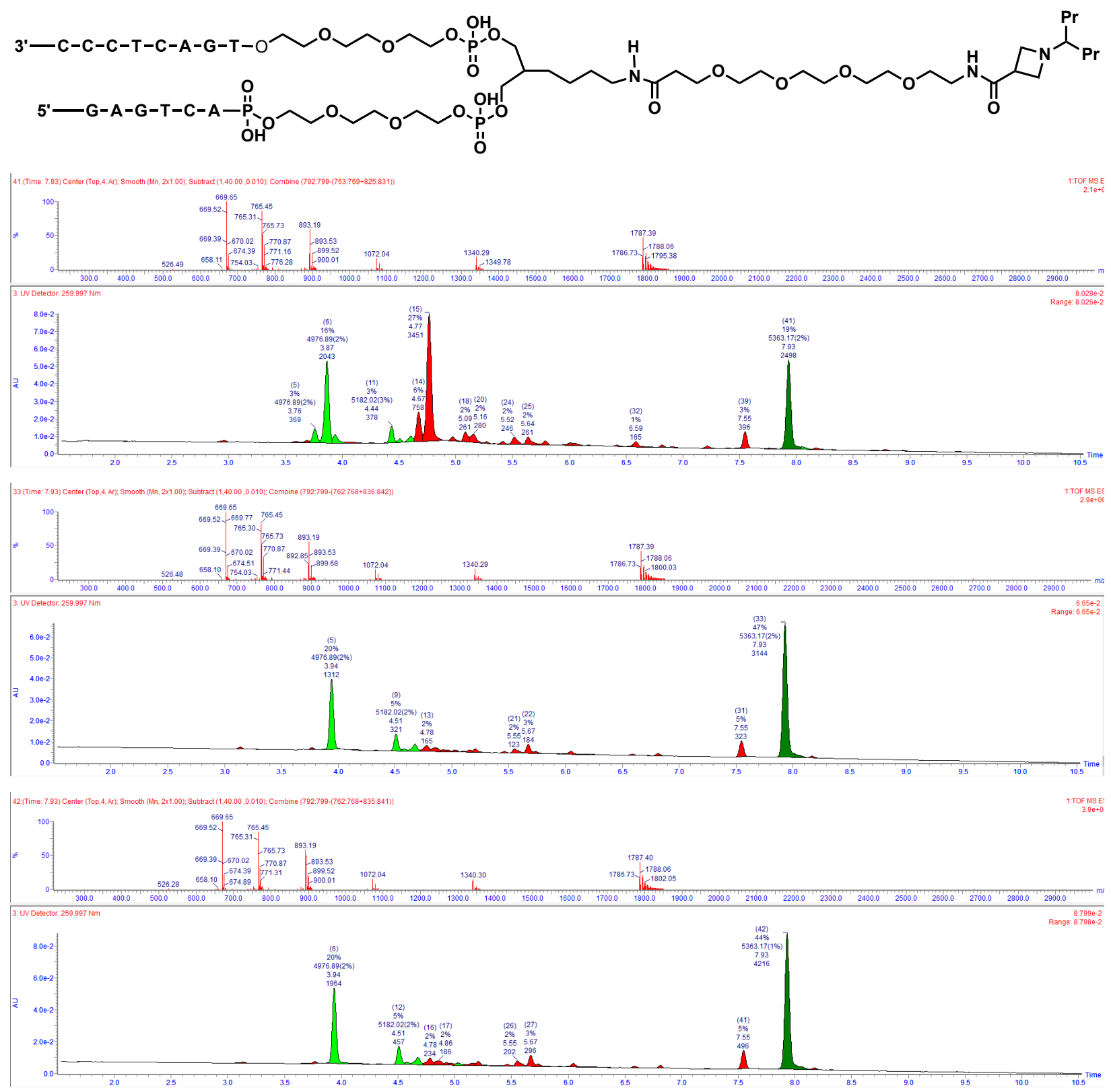


\section{LC Trace and Mass of 88 (Aqueous Reaction)}

Following General Procedure 7 with 66

Yields: $70 \%$

Exact masses: 5363.17

Triply charged masses [M-3]/3, caluculated: 1786.7; observed 1787.4
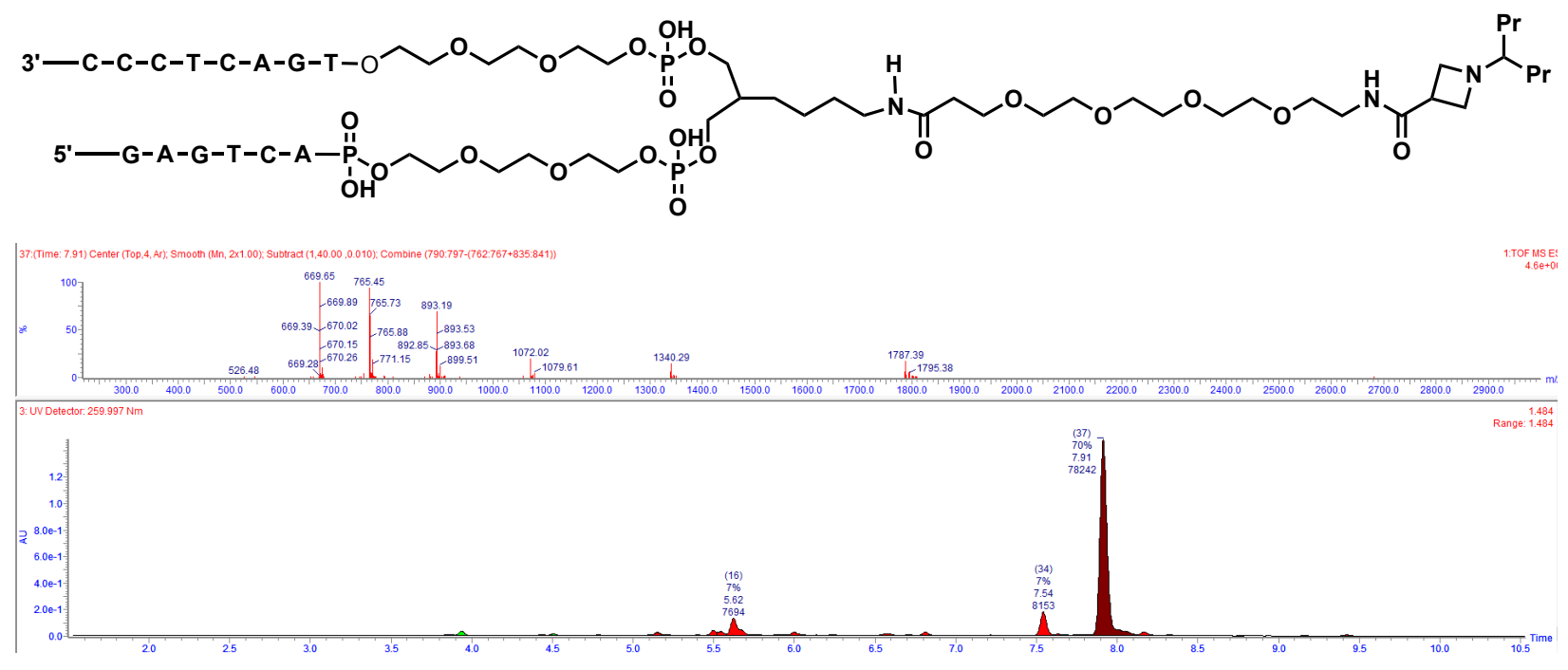
DEL Rehearsal Reaction Cycles—Synthesis of 90

\section{1. sp2-sp3 Cross Coupling}
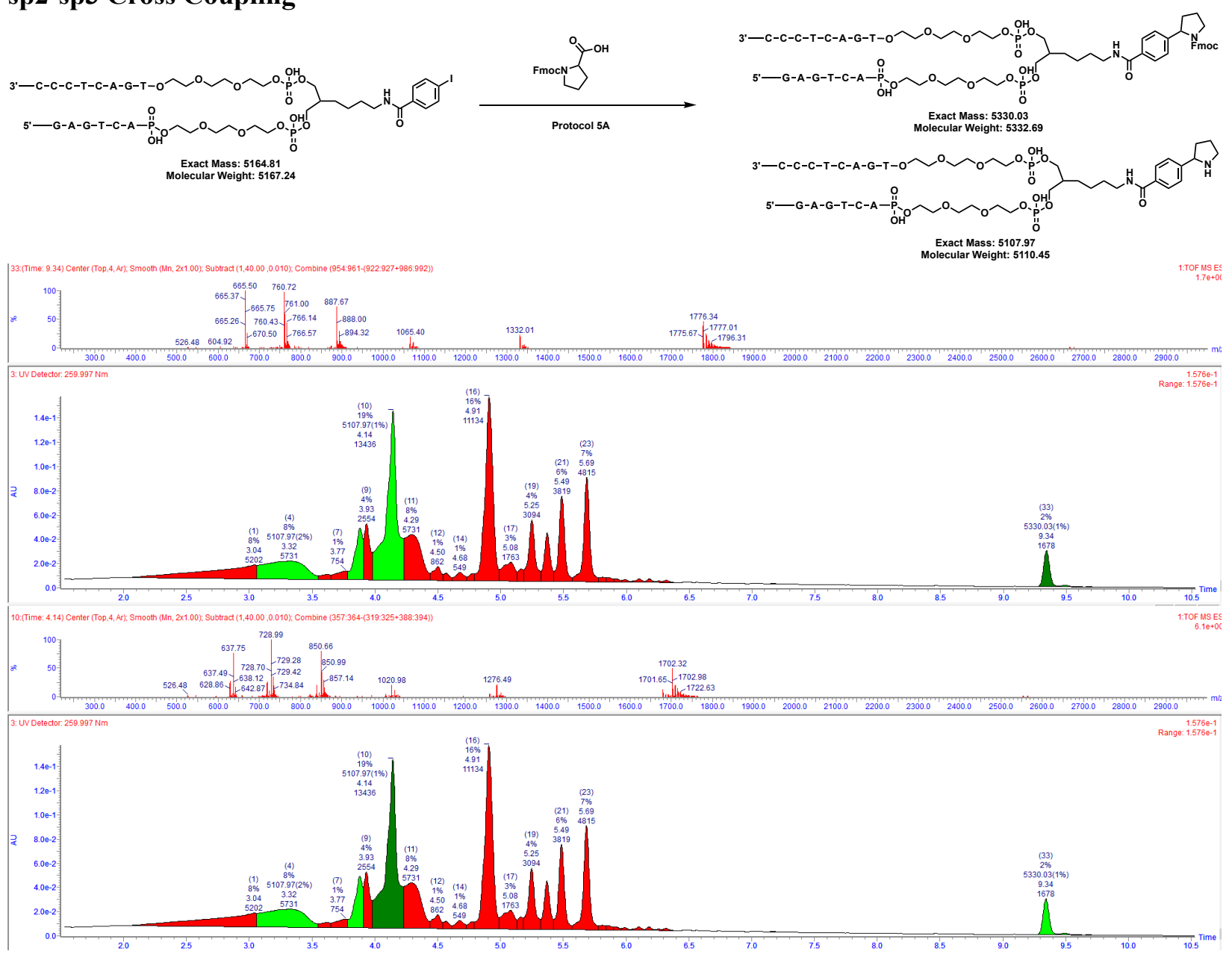


\section{Fmoc-Deprotection}
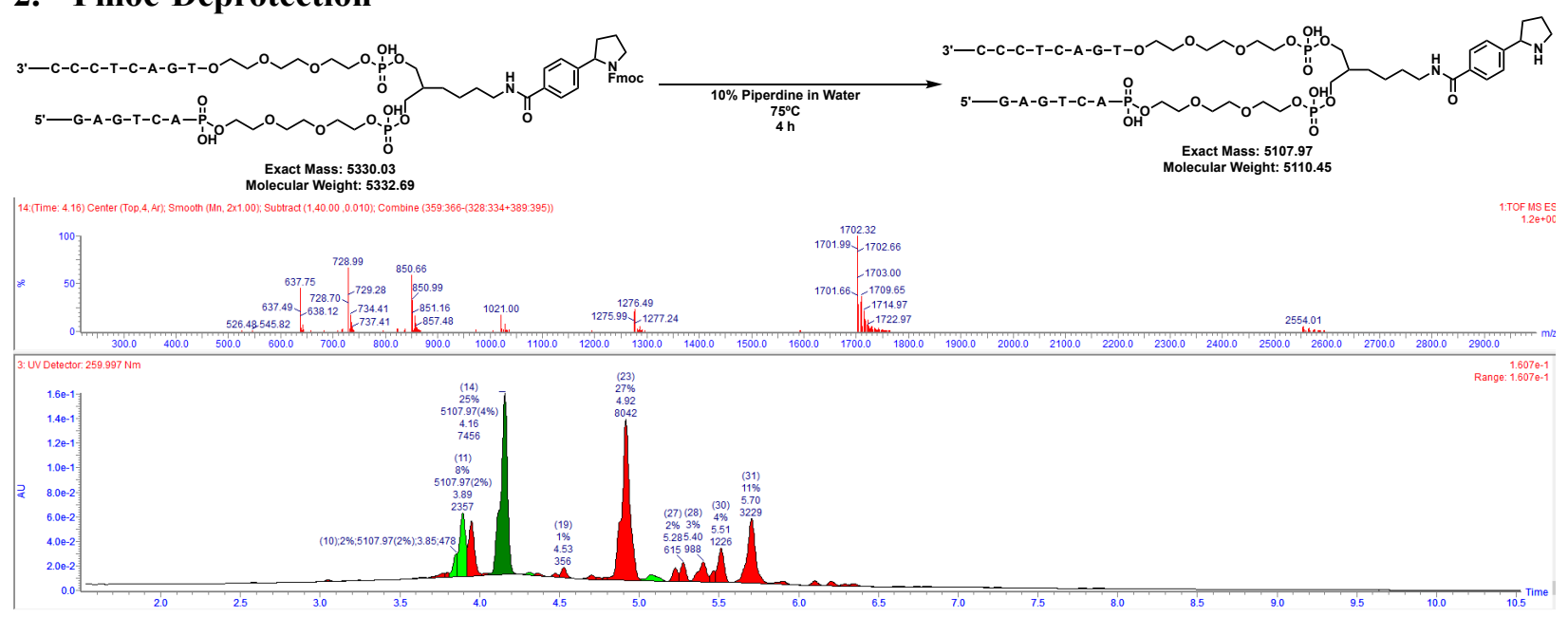

\section{Reductive Amination}
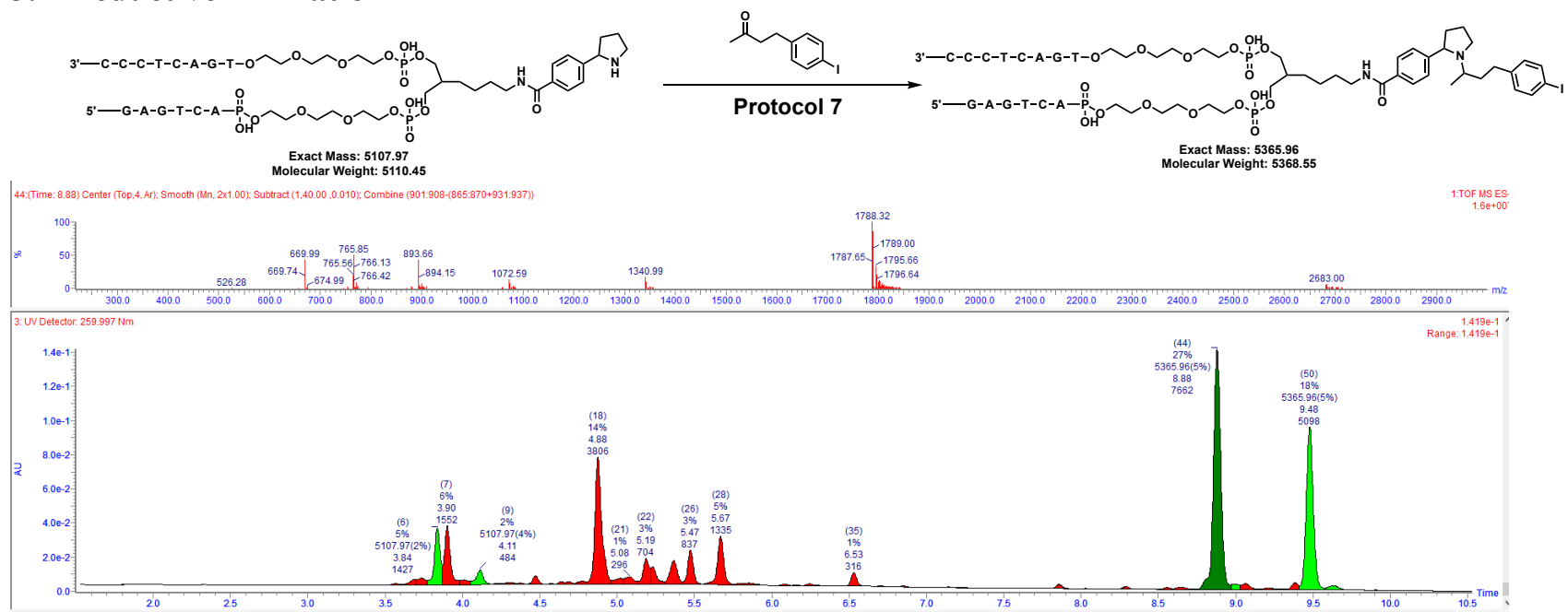

\section{Electrochemical Amination}
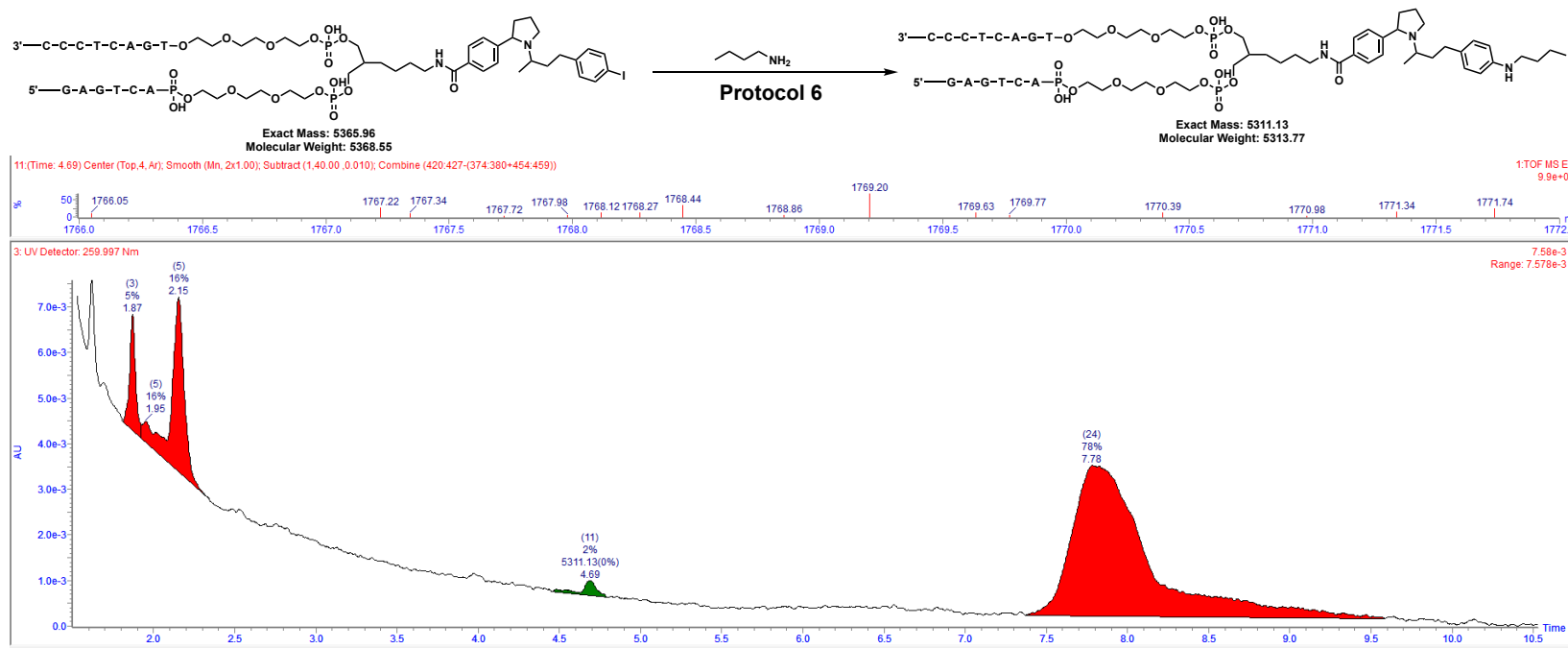


\section{Fluorescence Confocal Microscopy}

In order to test the elucidate the conformational state of the DNA while adsorbed to the resin bead, $100 \mu \mathrm{L}$ resin (Strata XAL) was treated with $50 \mathrm{nmol}$ double-stranded DNA, $100 \mathrm{nmol}$ single-stranded DNA, or no DNA in $100 \mu \mathrm{L}$ of PBS buffer and left shaking for 10 minutes to allow DNA to adhere to the resin. $1 \mu \mathrm{L}$ of SYBER Safe dye (100x), an intercalating fluorophore (Invitrogen SYBR Safe DNA gel stain) that preferentially binds dsDNA over ssDNA, was added to PBS buffer in all three conditions and left shaking for 10 min to stain DNA. The resin was spun down and the supernatant was removed from the resin. The resin was washed three times with $1 \mathrm{~mL}$ 1:1 PBS:Acetonitrile to remove any unbound dye. $10 \mu \mathrm{L}$ of each resin sample was added to a drop of ethanol on glass slides and secured with ProLong Gold Antifade Mountant. Fluorescence images were taken with a 20x objective on a Nikon A1 Confocal Microscope. The dye was excited at $488 \mathrm{~nm}$ and fluorescence was monitored at $525 \mathrm{~nm}$. Data was filtered to exclude areas smaller than 4000 square pixels, beads that were out of the plane of focus presented distorted intensity values. Mean intensity of each bead was calculated for 151 dsDNA beads, 281 resin beads, and 234 ssDNA beads using ImageJ. Using the Mann-Whitney rank test, since the distribution could not be assumed to be normal, the dsDNA distribution was found to be statistically significantly different from the ssDNA distribution $\left(p=5.64 \times 10^{-62}\right)$ and the resin distribution $\left(p=3.40 \times 10^{-64}\right)$. The resin and ssDNA distributions were not found to be significantly distinct $(\mathrm{p}=0.149)$. The higher intensity of staining of dsDNA on resin as compared to ssDNA on resin and resin only controls implies that DNA remains double stranded when adhered to the resin.

Sequence:

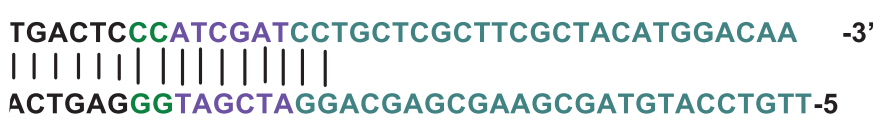

Mann Whitney U Test Results: dsDNA-ssDNA: $t=0.0 \mathrm{p}=5.638117765658832 \mathrm{e}-62$ dsDNA-res: $t=0.0 \mathrm{p}=3.400404067713507 \mathrm{e}-66$ res-ssDNA $: t=31132.0 \mathrm{p}=0.14$ 975837376795464 
Double Stranded DNA raw data:

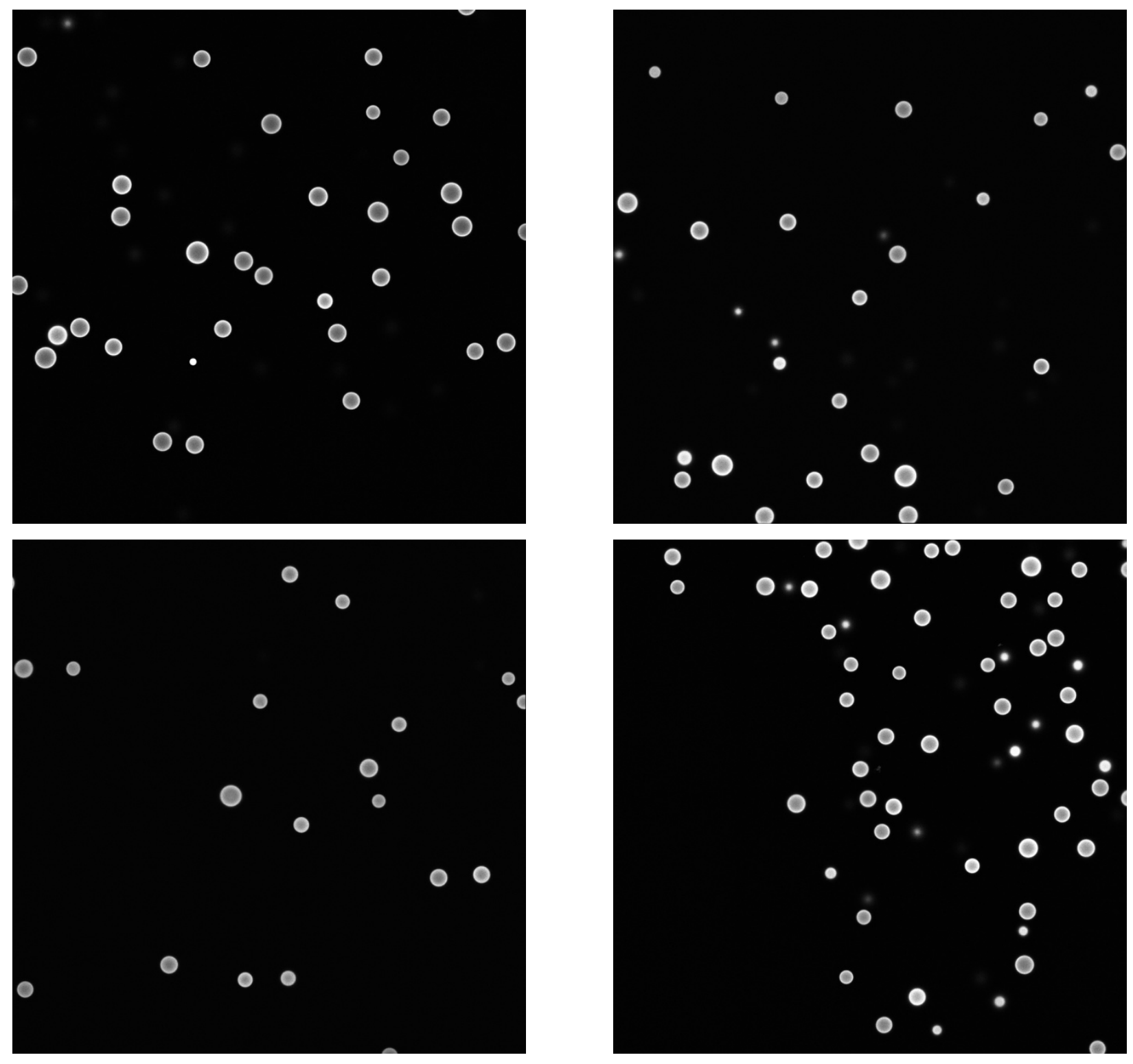




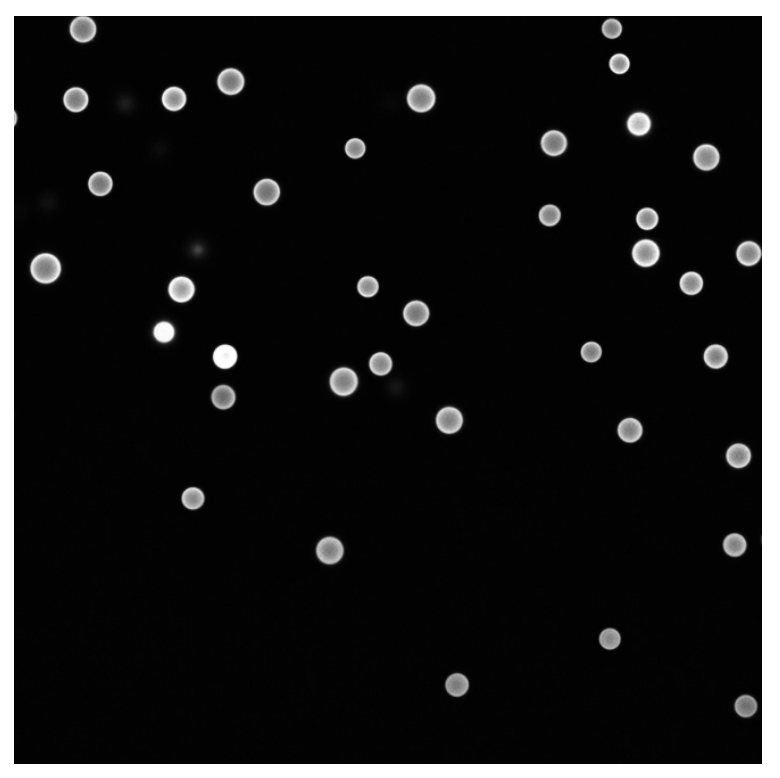


Single Stranded DNA raw data:
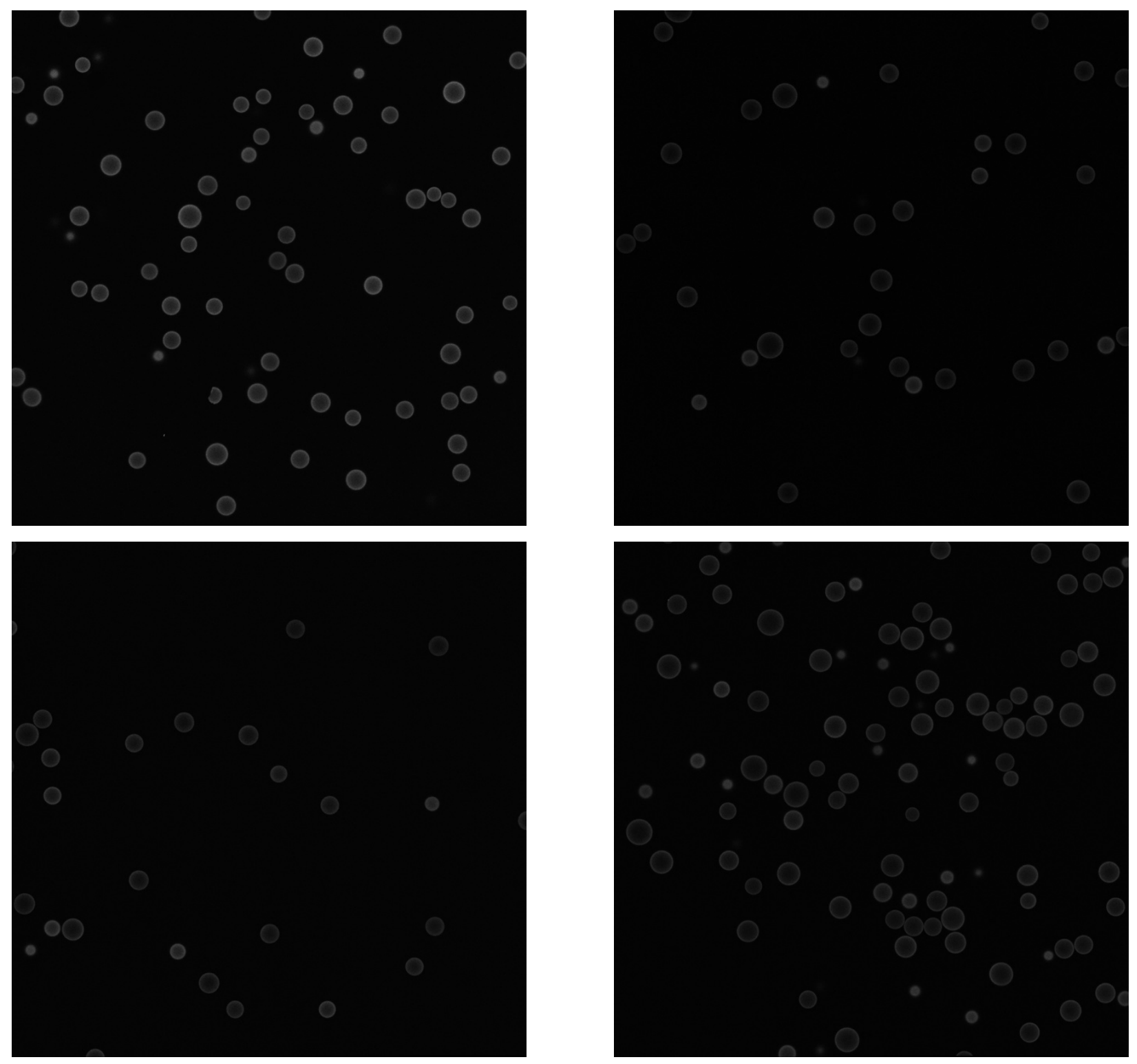


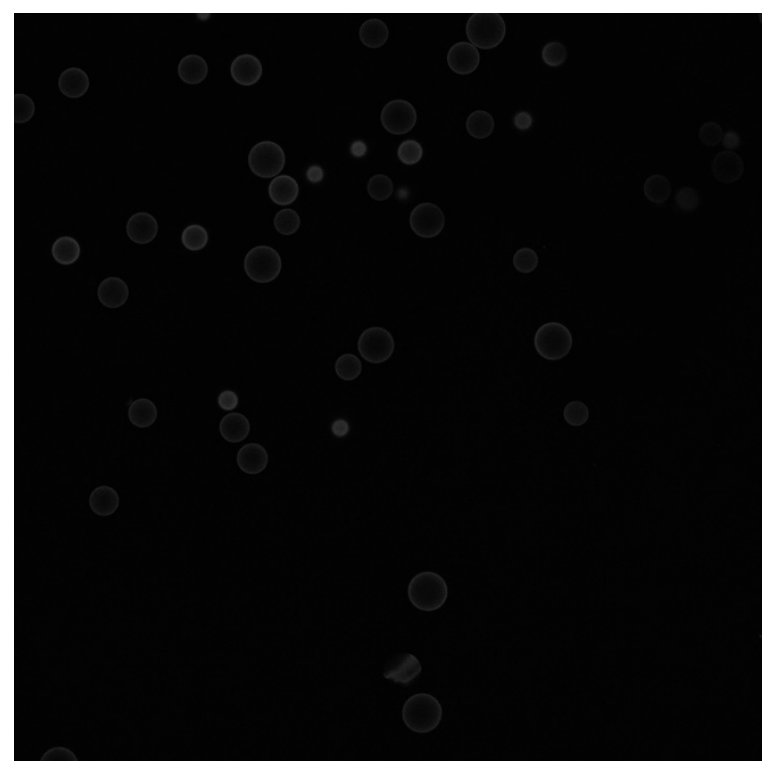


Resin only raw data:
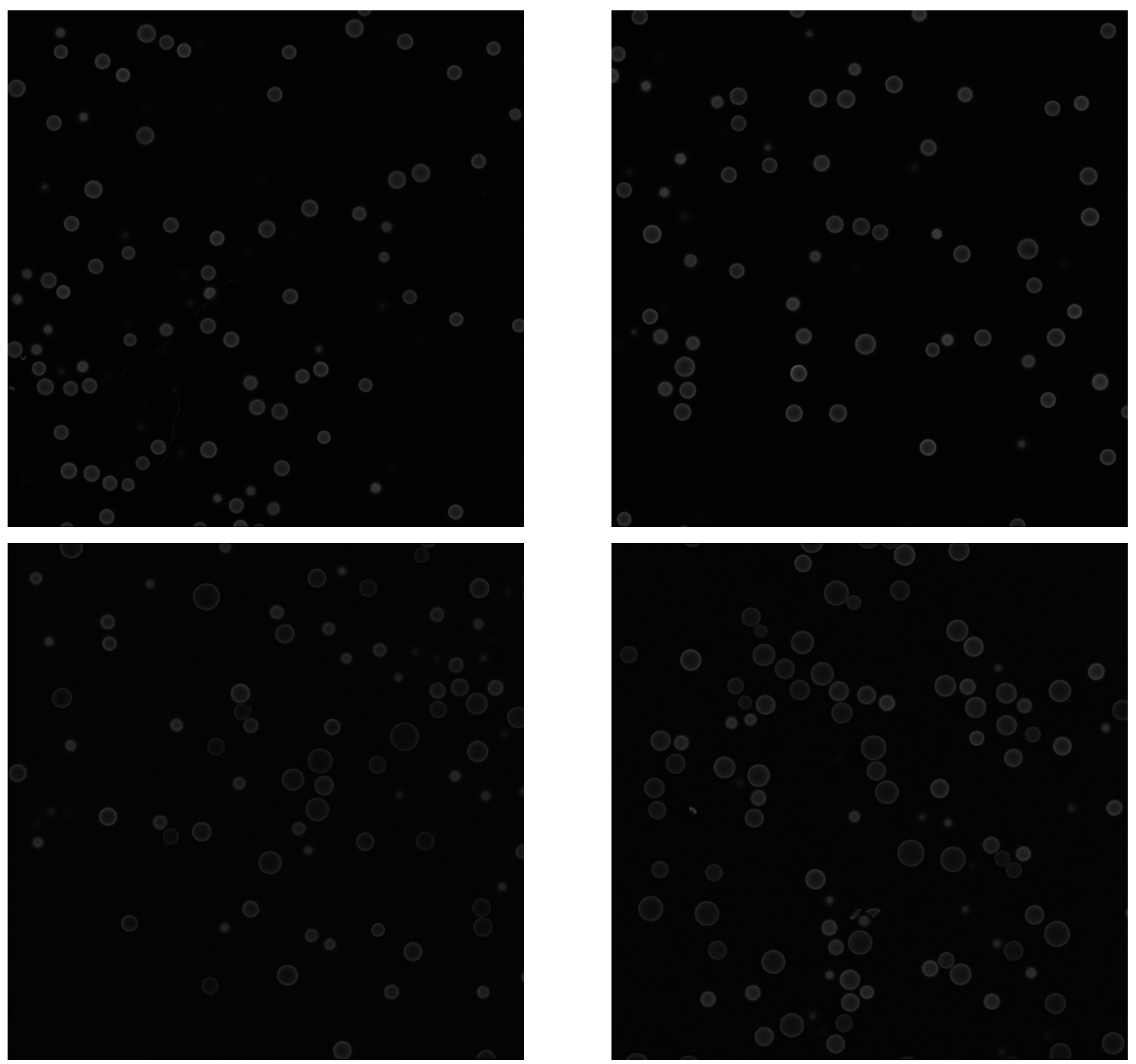


\section{Binding and Elution of 40mer DNA}

40mer DNA was bound and eluted as described about in Protocol 1 and 2. Analysis was performed by DNA Gel, as described on page 121 .

Sequence:

TGACTCCCATCGATCCTGCTCGCTTCGCTACATGGACAA $\quad-3^{\prime}$ || |||||||||||

ACTGAGGGTAGCTAGGACGAGCGAAGCGATGTACCTGTT-5
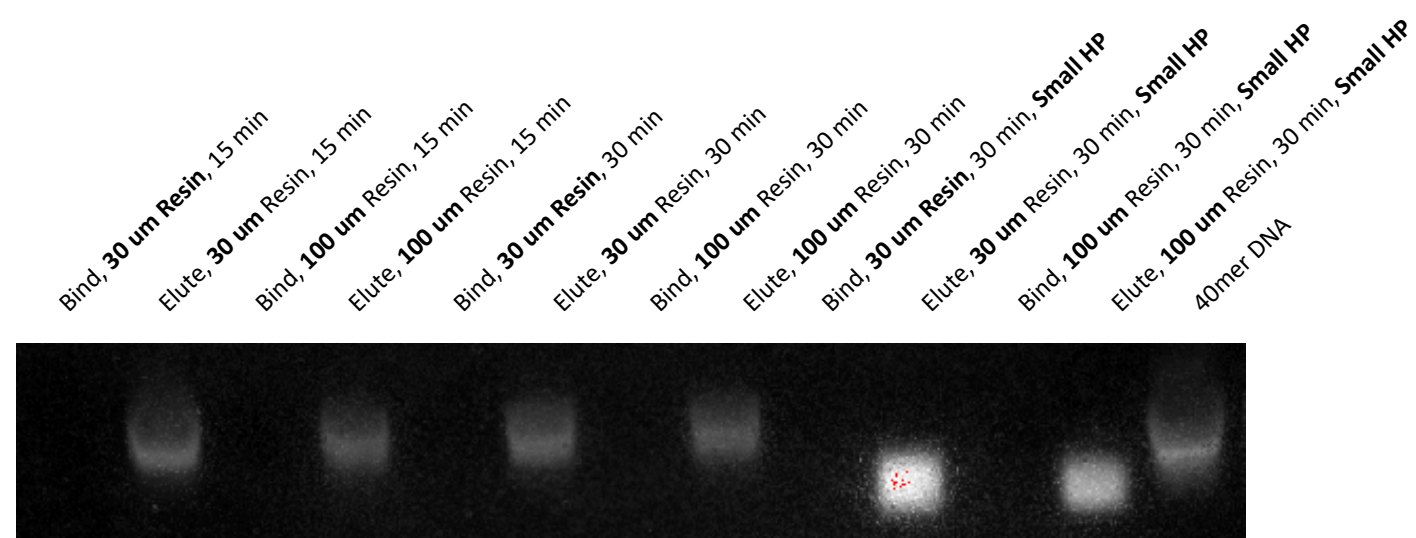


\section{References}

(1) Cornella, J.; Edwards, J. T.; Qin, T.; Kawamura, S.; Wang, J.; Pan, C. -M.; Gianatassio, R.; Schmidt, M.; Eastgate, M. D.; Baran, P. S. J. Am. Chem. Soc, 2016, 138, 2174.

(2) Qin, T.; Cornella, J.; Li, C.; Malins, L.; Edwards, J. T.; Kawamura, S.; Maxwell, B. D.; Eastgate, M. D.; Baran, P. S. Science, 2016, 352, 801.

(3) Wang, J.; Lundberg H.; Asai, S.; Martín-Acosta, P.; Chen, J. S.; Brown, S.; Farrell, W.; Dushin, R. G.; O’Donnel, C. J.; Ratnayake, A. S.; Richardson, P.; Liu, Z.; Qin, T.; Blackmond, D. G.; Baran, P. S. Proc. Natl. Acad. Sci. USA, 2018, 115, E6404.

(4) Ni, S.; Padial, N. M.; Kingston, C.; Vantourout, J. C.; Schmitt, D. C.; Edwards, J. T.; Kruszyk, M.; Merchant, R. R.; Mykhailiuk, P. K.; Sanchez, B.; Yang, S.; Perry, M.; Gallego, G. M.; Mousseau, J. J.; Collins, M. R.; Cherney, R. J.; Lebed, P. S.; Chen, J. S.; Qin, T.; Baran P. S. J. Am. Chem. Soc, DOI: $10.1021 /$ jacs.9b02238. 
